\title{
SIMULATION OF WAVEGUIDE CROSSINGS AND CORNERS WITH COMPLEX MODE MATCHING METHOD
}


SIMULATION OF WAVEGUIDE CROSSINGS AND CORNERS WITH

COMPLEX MODE MATCHING METHOD

By

Rui Wang, B.Sc., M.Sc.

\begin{abstract}
A Thesis
Submitted to the School of Graduate Studies

In Partial Fulfilment of the Requirements
\end{abstract}

For the Degree

Master of Applied Science

McMaster University

(C) Copyright by Rui Wang, August 2011 
MASTER OF APPLIED SCIENCE (2011)

(Electrical and Computer Engineering)
McMaster University

Hamilton, Ontario
TITLE:

Simulation of Waveguide Crossings and Corners with Complex Mode

Matching Method

AUTHOR:

Rui Wang, B.Sc. (Peking University, China)

SUPERVISOR:

Dr. Wei-Ping Huang

Professor, Department of Electrical and Computer Engineering 


\section{Abstract}

Optical waveguides are basic building blocks of high-density photonic integrated circuits and play crucial roles in optical access networks, biomedical system, sensors and so on. Various kinds of dielectric waveguides apply the total internal reflection condition to transmit optical field [9] and even more complicated structures based on waveguide interconnects, Bragg grating, photonic crystals are actively developed by corporations and academic institutes. Especially, the fast developing pace of Metal-Organic Chemical Vapor Deposition (MOCVD), Molecular Beam Epitaxy (MBE) and other fabrication techniques has predicted the increasing complication and thus more advanced function of modern optics integrated circuits. Under such circumstances, convenient and accurate modeling and simulation schemes are necessary for the exploration, designing and optimization of photonic devices, systems and networks before the time-consuming and expensive fabrication process.

The thesis summarizes several frequency-domain modeling schemes for the calculation of mode profile or beam propagation in 2D dielectric waveguide. The thesis mainly covers conventional Smooth Transition Method (STM), High Order Finite Difference (HOFD) scheme, Complex STM, and Complex Mode Matching Method (CMMM) based on the 2D waveguide model terminated with Perfect Matching Layer (PML) and Perfect Reflection Boundary (PRB). The mode spectrums and modal patterns obtained from Complex STM are compared with those of HOFD, 
and the simulation of waveguide crossings and corners with CMMM is validated with Finite-Difference-Time-Domain (FDTD) Method. 


\section{Acknowledgement}

I am grateful to my supervisor Dr. Weiping Huang for his guidance, instructions, great encouragement and understanding. I also should thank to the helpful discussions with

Dr.Xun Li. Further I hope to express my gratitude to Dr. Chih-Hung (James) Chen, Dr. Mohamed Bakr, Dr.Xun Li and Dr.Weiping Huang for their enlightening course lectures.

Many thanks to Cheryl Gies and Helen Jachna in the Department of Electrical and Computer Engineering for their nice help.

I am thankful to my colleagues, Jianwei Mu, Lin Han, Dong Zhou, Qingyi Guo, Yu Li, Lanxin Deng and Yanping Xi for their important advices.

At last I should appreciate sincerely my parents, and my undergraduate thesis advisor Dr.Yunyi Fu, for their ever support. 


\section{Contents}

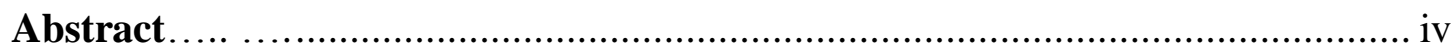

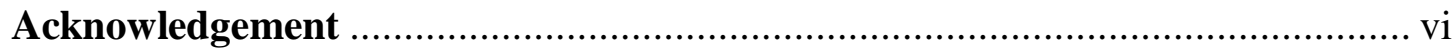

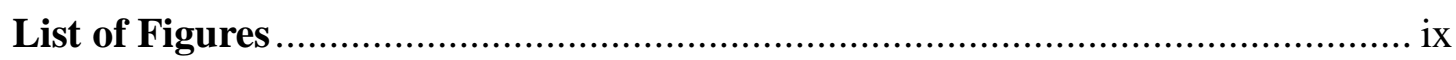

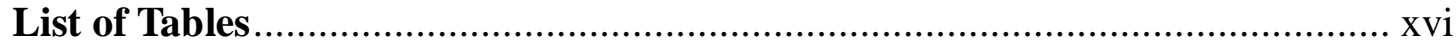

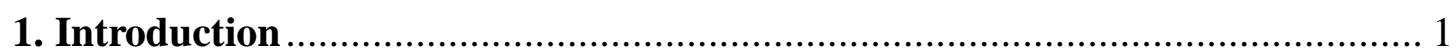

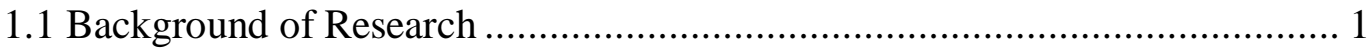

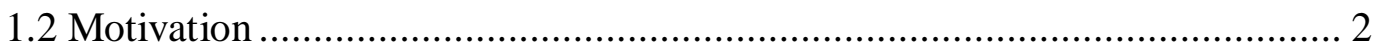

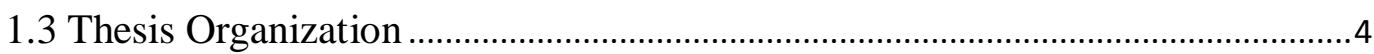

2. Finite Difference (FD) and Smooth Transition Method (STM) Solutions ......... 5

2.1 Mode Equation for 2D Straight Waveguide .............................................. 5

2.2 Conventional STM for 2D Straight Waveguide ......................................... 9

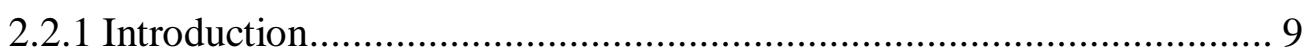

2.2.2 Formulas of Conventional STM ................................................... 10

2.3 Perfect Matching Layer as Numerical Boundary Condition ....................... 12

2.3.1 Discretized Box Modes ................................................................ 12

2.3.2 Modified 2-D Waveguide Governing Equation............................... 16

2.3.3 Hollow Waveguide Terminated with PML and PRB ..................... 22

2.4 High Order Finite Difference (HOFD) Scheme ....................................... 30

2.4.1 Formulas of HOFD Scheme …....................................................... 30

2.4.2 Validation of HOFD Scheme in Slab Waveguide .............................. 33

2.4.3 Validation of HOFD Scheme in Hollow Waveguide ................................ 37

3. Complex Mode Matching Method (CMMM) .......................................... 43

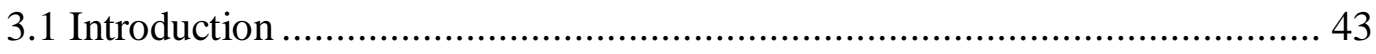

3.2 Transfer Matrix Formulas ...................................................................... 43

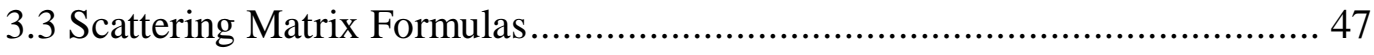

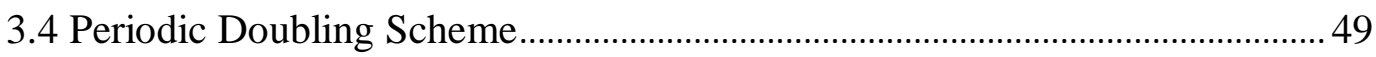

4. Complex Smooth Transition Method (STM) …......................................... 51 


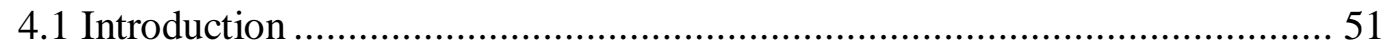

4.2 Formulas of Complex STM................................................................ 52

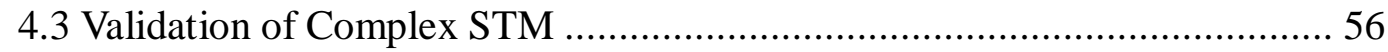

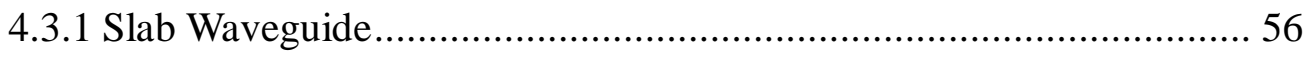

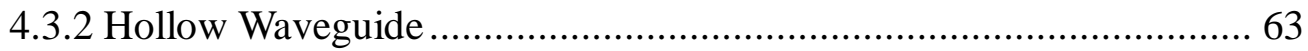

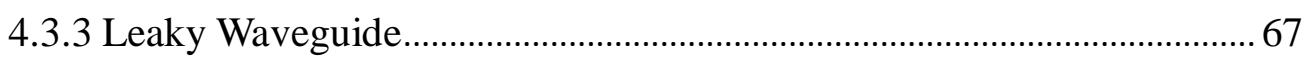

5. Power Conservation of Complex Mode Matching Method (CMMM)............ 71

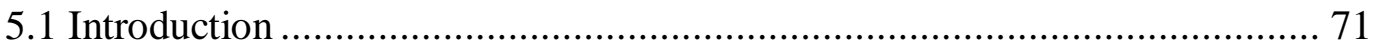

5.2 Analytical Validation in Hollow Waveguide ............................................... 71

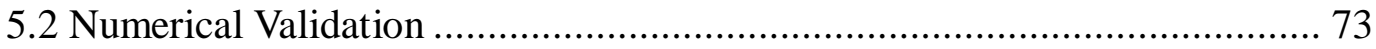

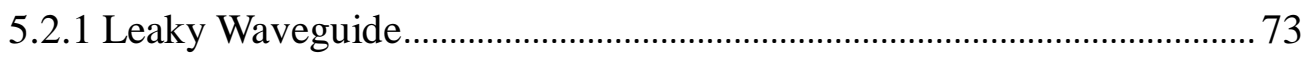

5.2.2 Waveguide Discontinuity ................................................................. 78

6. Simulation of Waveguide Crossings and Corners ....................................... 84

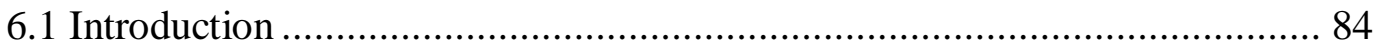

6.2 Simulation of High-Index-Contrast Waveguide Crossings and Corners ....... 85

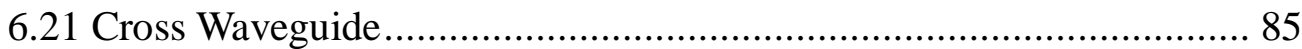

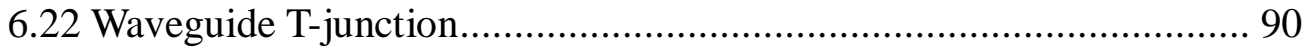

6.23 Waveguide Corner...................................................................... 93

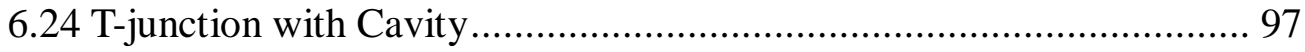

6.3 The Validation of CMMM with QDEEM in Cross Waveguide........................ 103

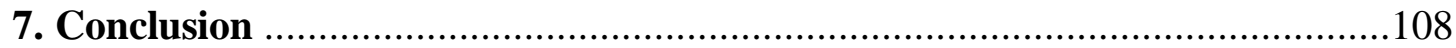

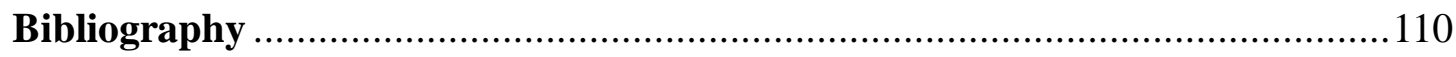

Appendix A List of Publications.................................................................. 114 


\section{List of Figures}

Figure 2.1. The schematic of an arbitrary 2-D slab waveguide

6

Figure 2.2. The schematic of a $(m+2)$-layer 2-D slab waveguide with artificial boundaries placed outside the multilayer stacks. ................................ 9

Figure 2.3. Methodology of Conventional STM................................................ 12

Figure 2.4. (a) 2-D open waveguide (b) 2-D close waveguide............................... 14

Figure 2.5. Mode spectrums of the closed symmetric slab waveguide in Figure

2.4(b) where $\mathrm{n} 1=3.3, \mathrm{n} 2=3.17,2 \mathrm{~S}=0.2 \mu \mathrm{m}$ for (a)TE polarization $\quad$ (b)

TM polarizations …........................................................................ 16

Figure 2.6. A 2-D slab waveguide enclosed with PML and PRB ......................... 17

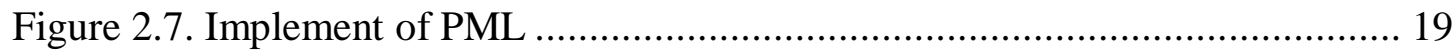

Figure 2.8. (a) The interface of waveguide region and PML (b) The interface of different PML layers.

Figure 2.9 The evolution of $\sigma_{x 0}$ with $R_{P M L}$ when $\mathrm{n} 2=3.17 . \mathrm{d}_{\mathrm{PML}}=5 \mu \mathrm{m}$. $\lambda=1.55 \mu \mathrm{m}$ in Figure 2.6(b)

Figure 2.10. The schematic of a 2-D hollow waveguide enclosed with PML and PRB on each side

Figure 2.11. The mode spectrums of hollow waveguides with different computation windows. $R_{P M L}=1 \mathrm{e}-2 . \quad \lambda=1.55 \mu \mathrm{m}$

Figure 2.12. The evolutions of $N_{\text {eff }}$ of (a) the $10^{\text {th }}$ even mode and (b) the $10^{\text {th }}$ odd mode with $R_{P M L}$ 26

Figure 2.13. The impact of $R_{P M L}$ on mode spectrums in hollow waveguide 27

Figure 2.14. The influence of $R_{P M L}$ on modal electric field patterns of (a) $1^{\text {st }}$ even mode, and (b) $1^{\text {st }}$ odd mode in hollow waveguide for TE polarization. 
Figure 2.15. The influence of $R_{P M L}$ on modal magnetic field patterns of (a) $1^{\text {st }}$ even mode and (b) $1^{\text {st }}$ odd mode in hollow waveguide for TM polarization.

Figure 2.16. Three 1-D adjacent sampled points for HOFD .................................. 31

Figure 2.17. $E_{y}$ patterns of (a) $\mathrm{TE}_{0},(\mathrm{~b}) \mathrm{TE}_{1}$ and (c)TE2 in slab waveguide 2......... 35

Figure 2.18. $H_{y}$ patterns of (a) $\mathrm{TM}_{0,}$ (b) $\mathrm{TM}_{1}$ and (c)TM2 in slab waveguide $2 \ldots \ldots 37$

Figure 2.19. Mode spectrums of in (a)TE and (b)TM cases in hollow waveguide .... 38

Figure 2.20. $E_{y}$ patterns of (a) the $2^{\text {nd }}$ even mode, (b) the $3^{\text {rd }}$ even mode, (c) the $2^{\text {nd }}$ odd mode and (d) the $3^{\text {rd }}$ odd mode in hollow waveguide for TE polarization.

Figure 2.21. ${ }^{H_{y}}$ patterns of (a) the $2^{\text {nd }}$ even mode, (b) the $3^{\text {rd }}$ even mode, (c) the $2^{\text {nd }}$ odd mode and (d) the $3^{\text {rd }}$ odd mode in hollow waveguide for TM polarization

Figure 3.1 The schematic of a waveguide discontinuity between 2-D waveguides

Figure 3.2. The schematic of two adjacent 2-D slab waveguide terminated with PML and PRB. $\mathrm{n} 1=3.3, \mathrm{n} 2=3.17, \mathrm{n} 3=3.4, \mathrm{n} 4=3.2, \mathrm{~d} 1=0.5 \mu \mathrm{m}, \mathrm{d} 2=2 \mu \mathrm{m}$, $\mathrm{dPML}=5 \mu \mathrm{m}$.

Figure 3.3. The continuity test of $E_{y}$ patterns at the waveguide junction (Figure

3.2) for TE polarization

Figure 3.4. The continuity test of $H_{y}$ patterns at the waveguide junction (Figure

3.2) for TM polarization 47

Figure 3.5. The schematic of a homogeneous 2-D region $\mathrm{C}$ 48

Figure 3.6. The schematic of a 2-D Bragg grating.... 50

Figure 4.1. The schematic of a multi-layer planar waveguide terminated with PML and PRB on both sides 53

Figure 4.2. Methodology of Complex STM 56 
Figure 4.3. Mode spectrums of (a) TE and (b) TM cases in slab waveguide 1 57

Figure 4.4. Modal field patterns in slab waveguide 1 (a) TE case. $N_{\text {eff }}$ for STM is $1.1412-12.4123 \mathrm{i}, \quad N_{\text {eff }}$ for FD is $1.1346-12.4096 \mathrm{i}$ (b) TM case. $N_{\text {eff }}$ for STM is $1.1468-12.4173 \mathrm{i}, \quad N_{\text {eff }}$ for FD is $1.1378-12.4149 \mathrm{i}$ 59

Figure 4.5. Mode orthogonality for complex STM in slab waveguide 1. (a) TE case (b) TM case 60

Figure 4.6. Mode spectrums of (a) TE and (b) TM case in slab waveguide 2 61

Figure 4.7. Modal field patterns of slab waveguide 2 (a) TE. $N_{\text {eff }}$ for complex STM is $0.4099-3.2799 \mathrm{i}, \quad N_{\text {eff }}$ for FD is $0.4135-3.4035 \mathrm{i}$ (b) TM. $N_{\text {eff }}$ for complex STM is $0.4775-5.5776 \mathrm{i}, \quad N_{\text {eff }}$ for FD is $0.4854-5.6794 \mathrm{i}$.

Figure 4.8. Mode orthogonality of complex STM in slab waveguide 2 (a) TE case (b) TM case 63

Figure 4.9. Mode spectrums of hollow waveguide (a) TE case (b) TM case 64

Figure 4.10. Modal field patterns of hollow waveguide (a) TE. $N_{\text {eff }}$ for complex STM is $0.4099-3.2797 \mathrm{i}, \quad N_{\text {eff }}$ for FD is $0.4135-3.4035 \mathrm{i}$ (b) TM. $\quad N_{\text {eff }}$ for complex STM is $0.4440-3.0675 \mathrm{i}, N_{\text {eff }}$ for FD is $0.4526-3.1232 \mathrm{i}$ 65

Figure 4.11. Mode orthogonality of complex STM in hollow waveguide (a) TE case (b) TM case 66

Figure 4.12. The schematic of a 2 -D leaky waveguide. $\mathrm{n}_{1}=3.17, \mathrm{n}_{2}=3.3, \mathrm{~d}_{1}=2 \mu \mathrm{m}$, $\mathrm{d} 2=2 \mu \mathrm{m}, \mathrm{dPML}=1 \mu \mathrm{m}$. 67

Figure 4.13. Mode spectrums of (a) TE and (b) TM cases in leaky waveguide 68

Figure 4.14. Modal field patterns of leaky waveguide (a) TE. $N_{\text {eff }}$ for STM is $0.6867-6.1270 \mathrm{i}, \quad N_{\text {eff }}$ for FD is $0.6907-6.1201 \mathrm{i}$. (b) TM. $N_{\text {eff }}$ for STM is $0.6956-6.2705 i, \quad N_{e f f}$ for FD is $0.7092-6.4068 \mathrm{i}$ 69 
Figure 4.15. Mode orthogonality of complex STM in leaky waveguide (a) TE case (b) TM case

Figure 5.1. The schematic of a 2-D hollow waveguide with a rectangular passive box in waveguide region

Figure 5.2. The schematic of a 2 -D step-index leaky waveguide.d $1=4 \mu \mathrm{m}$, $\mathrm{d} 2=2 \mu \mathrm{m}, \mathrm{d} 3=3 \mu \mathrm{m}, \mathrm{n} 1=2.2, \mathrm{n} 2=2, \mathrm{n} 3=1, \mathrm{dPML}=3 \mu \mathrm{m}$

Figure 5.3. (a) Modal field amplitude patterns for TE polarization (b) Convergence of $N_{\text {eff }}$ with $R_{P M L}$ 75

Figure 5.4. (a) Modal field amplitude patterns in TM polarization (b) Convergence of $N_{\text {eff }}$ with $R_{P M L}$ 76

Figure 5.5. Power conservation of CMMM in leaky waveguide (a) TE (b) TM. 77

Figure 5.6. The schematic of a junction of $2-\mathrm{D}$ waveguides. $\mathrm{dPML}=3 \mu \mathrm{m}, \mathrm{n} 1=3.4$, $\mathrm{n} 3=3.3, \mathrm{n} 2=1, \mathrm{n} 4=3.5, \quad \mathrm{~d} 1=1 \mu \mathrm{m}, \mathrm{d} 2=2 \mu \mathrm{m}, \mathrm{d} 3,4=2 \mu \mathrm{m} . \quad \mathrm{L} 1=2 \mu \mathrm{m}$, $\mathrm{L} 2=50 \mu \mathrm{m}$. 78

Figure 5.7. The convergence test of $P_{z, \text { out }}$ (a) about the computation window and (b) the number of modes applied for TE polarization in the waveguide junction (Figure 5.6)

Figure 5.8. The convergence test of $P_{z, \text { out }}$ (a) about the computation window and (b) the number of modes applied for TM polarization in the waveguide junction (Figure 5.6)

Figure 5.9. Power conservation of CMMM in the waveguide junction (Figure 5.6) (a) TE (b) TM

Figure 5.10. Transversely outgoing field amplitude patterns in the waveguide junction (Figure 5.6) (a) TE (b) TM

Figure 5.11. Vertically outgoing field amplitude pattern in the waveguide junction (Figure 5.6) (a) TE (b) TM.

Figure 6.1. The schematics of 2-D (a)waveguide crossing (b)waveguide T-junction (c)waveguide corner terminated with PML and PRB. 85 
Figure 6.2. Convergence test of guided crosstalk about (a) $R_{P M L}$ (b) the number of modes applied, and (c) the computation window in the high-index-contrast waveguide crossing .

Figure 6.3. Guided transmission and crosstalk spectra in the high-index-contrast waveguide crossing

Figure 6.4. The evolutions of guided transmission and crosstalk with the variation of background index in high-index-contrast waveguide crossing. 88

Figure 6.5. The evolutions of guided transmission and crosstalk with D2 in high- index-contrast waveguide crossing.

Figure 6.6. Electric field amplitude pattern in the high-index-contrast waveguide crossing.

Figure 6.7. Convergence test of guided transmission about (a) $R_{P M L}$ (b) the number of modes applied and (c) computation window in high-index-contrast waveguide T-junction

Figure 6.8. Guided transmission and reflection spectra in the high-index-contrast T-junction.

Figure 6.9. The evolution of guided transmission and reflection with the variation of background index in the high-index-contrast waveguide T-junction.

Figure 6.10. The evolution of guided transmission and reflection with the variation of $\mathrm{D}_{2}$ in the high-index-contrast waveguide $\mathrm{T}$-junction 92

Figure 6.11. Electric field amplitude pattern in the high-index-contrast T-junction. 93

Figure 6.12. Convergence of guided transmission about (a) $R_{P M L}$ (b) the number of modes applied and (c) the computation window in the high-index-contrast waveguide corner 94

Figure 6.13. Guided transmission and reflection spectra in the high-index-contrast waveguide corner 95 
Figure 6.14. The evolutions of guided transmission and reflection with the variation of background index in high-index-contrast waveguide corner.

Figure 6.15. The evolutions of guided transmission and reflection with the variation of $\mathrm{D} 2$ in high index-contrast waveguide corner 96

Figure 6.16. Electric field amplitude pattern in high-index-contrast waveguide corner. 96

Figure 6.17. (a)The schematic of a 2-D waveguide T-junction (b) The schematic of a cavity-assisted waveguide T-junction. $\mathrm{dPML}=5 \mu \mathrm{m}, \mathrm{S}_{1}=0.6 \mu \mathrm{m}$, $\mathrm{S}_{2}=0.6 \mu \mathrm{m}, \mathrm{S} 3=20 \mathrm{~nm}, \mathrm{~S}_{4}=0.2 \mu \mathrm{m}, \mathrm{S} 5=20 \mathrm{~nm}, \mathrm{~S}_{6}=0.2 \mu \mathrm{m}$ .98

Figure 6.18. Convergence test of guided crosstalk about (a) the number of modes applied (b) $R_{P M L}$ and (c) the computation window in waveguide T-junction (Figure 6.28(a))

Figure 6.19. Convergence test of guided crosstalk about (a) $R_{P M L}$ (b) the number of modes applied, and (c) the computation window in cavity-assisted waveguide T-junction (Figure 6.17(b))

Figure 6.20. Guided transmission, reflection and crosstalk spectra of the cavity-assisted T-junction (Figure 6.17(b)), and guided crosstalk spectra of the T-junction (Figure 6.17(a))

Figure 6.21. Electric field amplitude pattern in cavity-assisted T-junction (Figure 6.17(b)) (a) CMMM (b) FDTD

Figure 6.22. Convergence test of $\mathrm{P}_{\mathrm{D}}$ about (a) $R_{P M L}$ (b) the number of modes applied, and (c) the computation window for the waveguide crossing ([38]) in TE polarization.

Figure 6.23. Convergence test of $\mathrm{P}_{\mathrm{D}}$ about (a) $R_{P M L}$ (b) the number modes of applied, and (c) the computation window for the waveguide crossing ([38]) in TM polarization case. 
Figure 6.24. The evolutions of guided $\mathrm{P}_{\mathrm{T}}, \mathrm{P}_{\mathrm{R}}, \mathrm{P}_{\mathrm{U}}$ and $\mathrm{P}_{\mathrm{D}}$ with the variation of

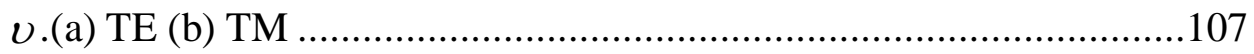




\section{List of Tables}

Table 1. The comparisons of guided mode $N_{\text {eff }}$ calculated from conventional STM and HOFD in slab waveguide (Figure 2.4(b)) with $\mathrm{n}_{1}=3.3$, $\mathrm{n}_{2}=3.17,2 \mathrm{~S}=2 \mu \mathrm{m}$, and $\lambda=1.55 \mu \mathrm{m}$ for TE polarization

Table 2. The comparisons of guided mode $N_{\text {eff }}$ calculated from conventional STM and HOFD in slab waveguide (Figure 2.4(b)) with n1=3.3, $\mathrm{n} 2=3.17,2 \mathrm{~S}=2 \mu \mathrm{m}$, and $\lambda=1.55 \mu \mathrm{m}$ for $\mathrm{TM}$ polarization

Table 3. Relative deviations between the sampled spectrums of CMMM and FDTD in high-index-contrast waveguide intersections

Table 4. Relative deviations of sampled transmission, crosstalk or reflection of CMMM and FDTD in high-index-contrast waveguide intersections with the background index varying ( $\mathrm{D}_{2}$ is $\left.0.2 \mu \mathrm{m}\right)$

Table 5. Relative deviations of sampled transmission, crosstalk or reflection of CMMM and FDTD in high-index-contrast waveguide intersections with D2 varying (the background index is 1) 


\section{Chapter 1 \\ Introduction}

\subsection{Background of Research}

Optical communication is a revolutionary and beneficial form of telecommunication using light as transmission medium and predicting high speed, broad bandwidth and good safety [1-2]. As a promising technology of the entire optical communication systems and networks, the concept of integrated photonics proposed in 1960s is supported by a variety of optical fibers and devices [3-4], among which waveguide crossings and corners are important components [5-8]. These structures are appealing due to their ability to control and direct the propagation of light in complex waveguide circuits. In a further way the scaling down of optical devices and circuits desires high-index-contrast waveguide structures because of the miniaturized waveguide core for optical confinement [9-10]. In this sense the designing and optimization of waveguide crossings and corners with high index-contrast have been widely studied experimentally and theoretically [11-17].

On the other hand, optical cavity attracted much attention owning to its capability of confining light through multiple internal reflections so as to produce standing waves for certain resonant frequencies. It has been reported that optical cavity can support longitudinal and transverse eigenmodes in the way of reproducing radiation patterns on every round-trip of the light through the resonator due to the 
effect of interference for certain patterns and radiation frequencies [18]. Optical cavity is generally employed in lasers [19-21], interferometers [22-23], wavelength filters [24-25] and so on. Especially optical cavities with appropriate geometry are usually applied to waveguide crossings and corners in order to enhance or suppress the crosstalk [11-12][26].

Computer-aided design (CAD) schemes suggested to simulate the wave propagation in waveguide structures ranges from semi-analytical methods, say Coupled Mode Theory (CMT) [27-30], and Mode Matching Method (MMM) [31-32], to numerical approaches like Beam Propagation Method (BPM) [33-34] and Finite-Difference Time-Domain (FDTD) Method [35-36]. However, in the past only computationally expensive time-domain schemes are universally employed for the modeling of waveguide crossings and corners [11], as the outgoing wave is actually radiating wave with reference to the launching direction so that the modeling of couplings among ports with different directions is challenging for frequency-domain method.

\subsection{Motivation}

Although the modeling of waveguide crossings and corners through FDTD is rigorous and accurate, it is extremely demanding of FDTD on computation time and memory [37]. Recent progress in the MMM utilizing the combination of PML and PRB have been proved to be effective and accurate through various examples such as waveguide facets, polarization rotators, and deep-etched gratings, etc. However All these 
structures bear the similar characteristics, i.e., the main radiation field out of the input waveguides is dominant in the directions not too far off the waveguide axis. As such, the guided and the near-axis complex modes with relatively lower radiation loss play significant roles in the mode matching simulation. So far, few frequency domain methods can handle radiation far off-axis or perpendicular to the waveguide axis as this involves fast oscillating evanescent waves. Although Quadri-Directional Eigenmode Expansion Method (QDEEM) has been supposed to simulate waveguide intersections, the orthogonal condition of this method is not clear [38]. In this work, we demonstrated by way of simulation that the total powers along all directions are conserved within the framework of CMMM in order to validate the self-consistency of the method. Further, we for the first time prove that CMMM is able to model the couplings of radiating wave perpendicular to the waveguide axis in the examples of waveguide crossings and corners.

High Order Finite Difference (HOFD) method has been a general numerical mode solver for 2-D straight waveguide terminated with PML and PRB. However, in HOFD the process of obtaining eigenvalues of the Hermitan Matrix in sophisticated waveguides requiring very small meshes costs time and memories a lot [39]. In this thesis the author develops the semi-analytical Complex Smooth Transition Method (CSMT) for the mode solver of multi-layer planar waveguide instead, and compares the obtained mode spectrums and modal patterns with those from HOFD scheme. 


\subsection{Thesis Organization}

The arrangement of this thesis is as follows: In Chapter 2, the modal governing equations and the solutions of both finite difference and conventional smooth transition are presented. Chapter 3 demonstrates the complex mode matching method. Chapter 4 describes the novel complex smooth transition method. The power conservation of complex mode matching method is proved in Chapter 5. Chapter 6 introduces the simulation of waveguide crossings and corners with complex mode matching method. And the conclusion of this thesis is drawn on Chapter 7.

The main contributions of this thesis include two points: firstly, we originally establish Complex STM to semi-analytically calculate the mode profiles of multi-layer planar waveguide terminated with both PML and PRB ; secondly, although CMMM has been generally applied to the simulation of waveguide facets, Bragg gratings, etc[52-53], we for the first time demonstrate that CMMM can also be utilized for the modeling of couplings of radiation field outgoing perpendicularly to the waveguide axis with an incident wave launched in the examples of high-index-contrast waveguide crossings and corners. CMMM is proved to be able to estimate the field profiles and power flows accurately through the validation with FDTD. 


\section{Chapter 2}

\section{Finite-Difference (FD) and Smooth Transition Method (STM) Solutions}

\subsection{Mode Equation for 2D Straight Waveguide}

In a source-free and homogeneous media, the Maxwell's equation can be written as [28]

$$
\begin{gathered}
\nabla \times \vec{E}=-j \omega \mu_{0} \vec{H} \\
\nabla \times \vec{H}=j \omega_{\mathcal{E}_{0} \varepsilon_{r}} \vec{E} \\
\nabla \cdot\left(\varepsilon_{0} \varepsilon_{r} \vec{E}\right)=0 \\
\nabla \cdot\left(\mu_{0} \vec{H}\right)=0
\end{gathered}
$$

where $\omega$ is the frequency of the light wave, $\varepsilon_{0}$ and $\mu_{0}$ are the permitivity and the permeability of the free space, respectively. The time harmonic factor $e^{j \omega t}$ has been suppressed in the time-harmonic Maxwell' s equations above.

Substituting (2.1) into (2.2), we can obtain the full vector wave equations with reference to electric field [28]:

$$
\nabla \times[\nabla \times \vec{E}]-\omega^{2} \mu_{0} \varepsilon_{0} n^{2} \vec{E}=0
$$

In the similar way, by taking (2.2) into (2.1), we get the full vector wave equation on magnetic field [28]

$$
\nabla \times\left[\frac{1}{n^{2}} \nabla \times \vec{H}\right]-\omega^{2} \mu_{0} \varepsilon_{0} \vec{H}=0
$$

where $n$ is the refractive index of the medium. 
Figure 2.1 shows a $2-\mathrm{D}$ slab waveguide. $\hat{Z}$ is the longitudinal direction, and $\hat{X}$ is the transverse direction. The waveguide along $\hat{Y}$ is infinitely long, thus $\frac{\partial}{\partial y}=0$. Consequently (2.5) and (2.6) will fall into two separate transverse electric mode (TE) equations and transverse magnetic mode (TM) equations.

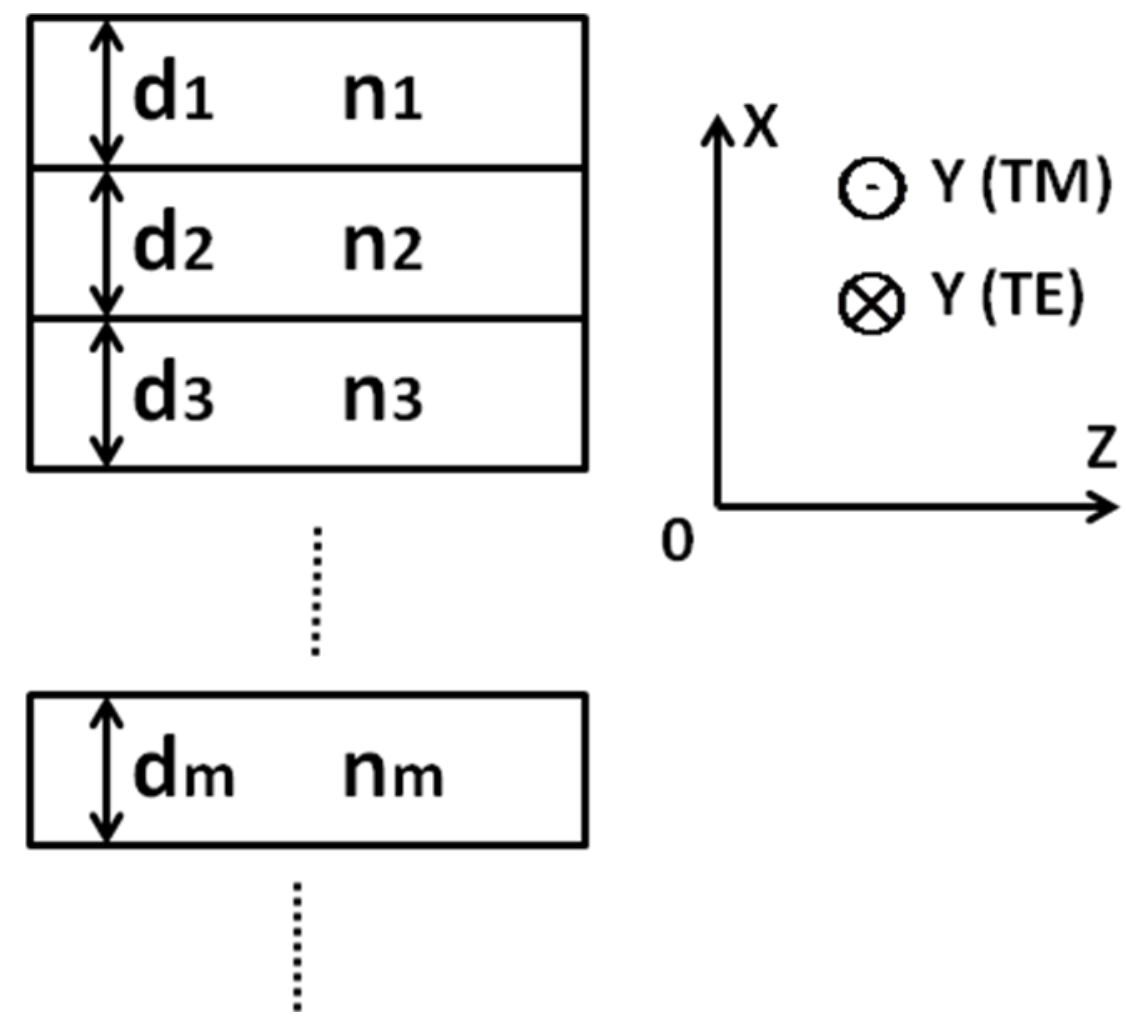

Figure 2.1. The schematic of an arbitrary 2-D slab waveguide

For TE polarization the wave vector only contains the components of $E_{y}$, $H_{z}$, and $H_{x}$. And the governing equations for $E_{y}$ can be simplified to be [27-28]:

$$
\left\{\begin{array}{c}
\frac{\partial^{2} E_{y}}{\partial x^{2}}+\left(k_{0}^{2} n^{2}-\beta^{2}\right) E_{y}=0 \\
\frac{\partial E_{y}}{\partial z}=-j \beta E_{y}
\end{array}\right.
$$

where $k_{0}$ is the vacuum wave number 


$$
k_{0}=\frac{2 \pi}{\lambda}
$$

$\beta$ here is the longitudinal propagation constant.

$H_{x}$ and $H_{z}$ are related to $E_{y}$ in (2.9) and (2.10) [27-28]

$$
\begin{aligned}
& H_{x}=-\frac{\beta}{\omega \mu_{0}} E_{y} \\
& H_{z}=\frac{j}{\omega \mu_{0}} \frac{\partial E_{y}}{\partial x}
\end{aligned}
$$

For TM polarization, the vector wave only contains the components of $H_{y}$, $E_{x}$ and $E_{z}$. The governing equation for $H_{y}$ is [27-28][30]:

$$
\left\{\begin{array}{c}
n^{2}\left[\frac{\partial}{\partial x}\left(\frac{1}{n^{2}} \frac{\partial H_{y}}{\partial x}\right)\right]+\left(k_{0}^{2} n^{2}-\beta^{2}\right) H_{y}=0 \\
\frac{\partial H_{y}}{\partial z}=-j \beta H_{y}
\end{array}\right.
$$

$E_{x}$ and $E_{z}$ can be expressed by $H_{y}$ in (2.12) and (2.13)

$$
\begin{gathered}
E_{x}=\frac{\beta}{\omega \varepsilon_{0} \varepsilon_{r}} H_{y} \\
E_{z}=\frac{j}{n^{2} \varepsilon_{0} \omega} \frac{\partial H_{y}}{\partial x}
\end{gathered}
$$

When the waveguide is enclosed by perfect boundary conditions (PRB) which result in the radiation field totally reflected at the boundaries, the continuous radiation field will be discretized into box modes. For general media, two arbitrary modes of the same waveguide are always orthogonal in this way [30] :

$$
\int\left(\vec{e}_{t m} \times \vec{h}_{t n}^{*}+\vec{e}_{t n}^{*} \times \vec{h}_{t m}\right) \cdot \vec{z} d x
$$

where $\vec{e}_{t m}$ and $\vec{h}_{t m}$ are the transverse electric and magnetic field of the $\mathrm{m}^{\text {th }}$ forward propagating mode. $\vec{e}_{t n}$ and $\vec{h}_{t n}$ are defined in the same way $\left(\beta_{m} \neq \beta_{n}\right)$. The integration here is along the whole cross section. 
We suppose $\beta_{m}$ as the propagation constant of the $\mathrm{m}^{\text {th }}$ forward propagation mode. When the $\mathrm{m}^{\text {th }}$ mode propagates backward, its propagation constant will be $-\beta_{m}$. Then its orthogonality with the $\mathrm{n}^{\text {th }}$ mode can be expressed as [30]:

$$
\int\left(\vec{e}_{t m} \times \vec{h}_{t n}^{*}-\vec{e}_{t n}^{*} \times \vec{h}_{t m}\right) \cdot \vec{z} d x, \quad \beta_{m} \neq-\beta_{n}
$$

In real waveguide structures, the transverse field pattern can be approximately expanded by the linear superposition of its eigenmodes, so-called mode expansion [29-30].

$$
\begin{aligned}
& \vec{E}_{t}(x, z)=\sum_{n=1}^{N}\left(a_{n}^{+} e^{-j \beta_{n} z}+a_{n}^{-} e^{j \beta_{n} z}\right) \vec{e}_{t n}(x) \\
& \vec{H}_{t}(x, z)=\sum_{n=1}^{N}\left(a_{n}^{+} e^{-j \beta_{n} z}-a_{n}^{-} e^{j \beta_{n} z}\right) \vec{h}_{t n}(x)
\end{aligned}
$$

where $a_{n}^{+}$and $a_{n}^{-}$are the amplitudes of the forward and backward propagating $n^{\text {th }}$ mode of the local waveguide.

In this mode expansion process, it is necessary to normalize the $n^{\text {th }}$ mode field pattern [30]

$$
\frac{1}{2} \int \operatorname{Re}\left(\vec{e}_{t n}(x) \times \vec{h}_{t n}^{*}(x)\right) \cdot \vec{z} d x=1
$$

For lossless media, the modal field is real. So the orthogonality condition can be simplified to be [30]

$$
\int\left(\vec{e}_{t m} \times \vec{h}_{t n} \pm \vec{e}_{t n} \times \vec{h}_{t m}\right) \cdot \vec{z} d x=0, \quad \beta_{m} \neq \pm \beta_{n}
$$

And the mode normalization expression turns to be [30]

$$
\frac{1}{2} \int \vec{e}_{t n}(x) \times \vec{h}_{t n}(x) \cdot \vec{z} d x=1
$$

For general media, the modal field pattern can be complex, accordingly in (2.21) $N_{n}$ can be even complex [30]

$$
\frac{1}{2} \int \operatorname{Re}\left(\vec{e}_{t n}(x) \times \vec{h}_{t n}^{*}(x)\right) \cdot \vec{z} d x=N_{n}
$$




\subsection{Conventional STM for 2-D Straight Waveguide}

\subsection{Introduction}

The determination of both guided modes and leaky modes are essential for the simulation, analysis, design and optimization of the multilayer planar waveguide structure, as leaky modes are usually critical to assist the analysis of mode couplings. In conventional STM, the cascading of transfer matrices leads to the characteristics equation $f(\beta)=0$, whose roots can be obtained by Newton's search in complex plane [40-41]. When the artificial boundary outside the multilayer stacks (Figure 2.2) makes the multilayer structure closed, we obtain the real and imaginary roots of $f(\beta)=0$ that are corresponding to the guided and evanescent modes, and they serve as the initial guesses for the following root searching process. As the artificial boundary changes from close to open, the root locations move from real and imaginary axis to the complex plane.
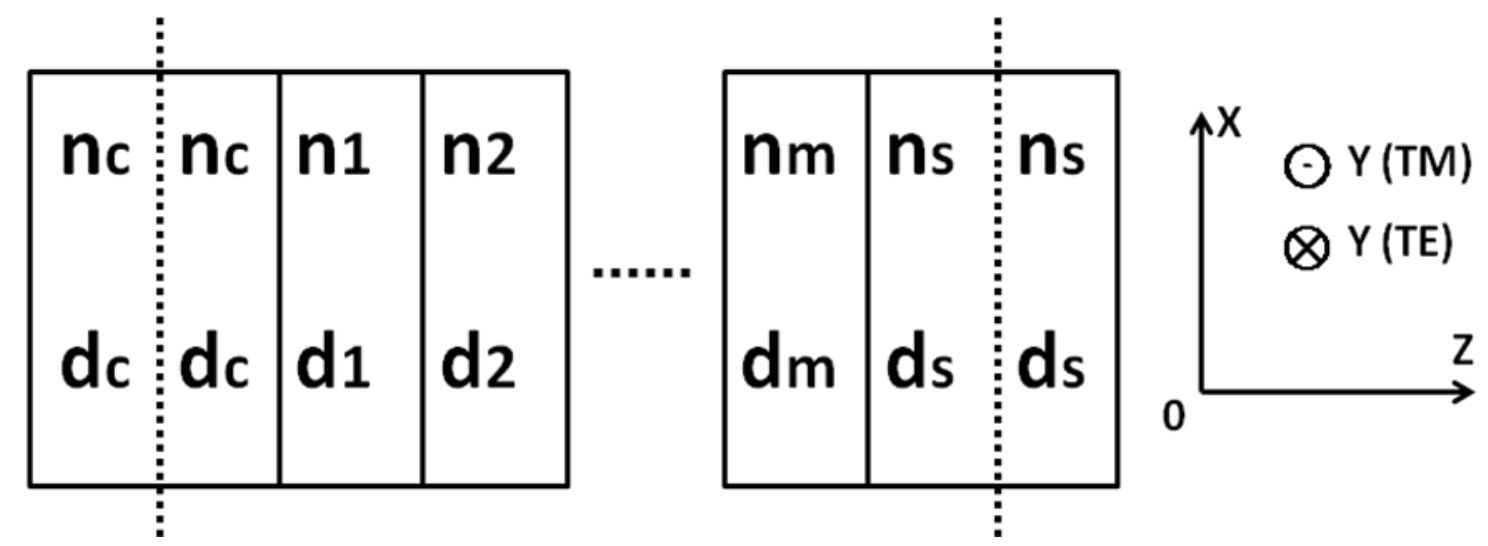

Figure 2.2. The schematic of a $(m+2)$-layer 2-D slab waveguide with artificial boundaries placed outside the multilayer stacks. 


\subsection{Formulas of Conventional STM}

The field expression in an arbitrary location in the $j^{\text {th }}$ layer in the multilayer structure of Figure 2.2 can be expressed as [40-41]:

$$
\varphi_{j}(x)=F_{j} e^{-j k_{j}\left(x-x_{j}\right)}+B_{j} e^{j k_{j}\left(x-x_{j}\right)}
$$

We get the derivation of field pattern with reference to $\mathrm{x}$

$$
\frac{\partial \varphi_{j}(x)}{\partial x}=-j k_{j}\left[F_{j} e^{-j k_{j}\left(x-x_{j}\right)}-B_{j} e^{j k_{j}\left(x-x_{j}\right)}\right]
$$

where $F_{j}$ and $B_{j}$ are the complex coefficient of forward and backward propagating waves at the left boundary of $j^{\text {th }}$ layer. $\varphi_{j}$ notes $E_{y}$ for TE case and does $H_{y}$ for TM case. $x$ is an arbitrary transverse position in the $j^{\text {th }}$ layer. $x_{j}$ is the left boundary of $j^{\text {th }}$ layer. The transverse wave vector $k_{j}$ is defined as

$$
k_{j}=\sqrt{n_{j}^{2} k_{0}^{2}-\beta^{2}}
$$

According to the Maxwell's equation in Chapter 2.1, in TE polarization, $\varphi_{j}(x)$ and $\frac{\partial \varphi_{j}(x)}{\partial x}$ vary as tangential electric and magnetic field, respectively. In TM polarization, $\varphi_{j}(x)$ and $\frac{1}{n_{j}^{2}} \frac{\partial \varphi_{j}(x)}{\partial x}$ vary as tangential magnetic and electric field.

We assume $F_{L}$ and $B_{L}$ are the complex coefficient of forward and backward propagating tangential electric (magnetic) field in TE (TM) polarization at the left artificial boundary; $F_{R}$ and $B_{R}$ are those at the right artificial boundary. Through the cascading of transfer matrix in each layer, we can get the equation (2.25) in TE polarization case [40-41].

$$
\left|\begin{array}{c}
F_{L}+B_{L} \\
k_{c}\left(F_{L}-B_{L}\right)
\end{array}\right|=\left|\begin{array}{cc}
T_{11} & T_{12} \\
T_{21} & T_{22}
\end{array}\right|\left|\begin{array}{c}
F_{R}+B_{R} \\
k_{s}\left(F_{R}-B_{R}\right)
\end{array}\right|
$$


We further define a controlling parameter $\gamma_{[40-41]}$

$$
\begin{gathered}
F_{L}-B_{L}=-\gamma\left(F_{L}+B_{L}\right) \\
F_{R}-B_{R}=\gamma\left(F_{R}+B_{R}\right)
\end{gathered}
$$

When $\gamma=1, F_{L}=0, B_{L} \neq 0 ; F_{R} \neq 0, B_{R}=0$, the artificial boundaries on both sides are open. As $\gamma$ gradually changes from 1 to $0, F_{L}=B_{L}, F_{R}=B_{R}$, the artificial boundaries are closed. Substituting (2.26) and (2.27) into (2.25), we get [40-41]

$$
\left|\begin{array}{c}
F_{L}+B_{L} \\
-\gamma k_{c}\left(F_{L}+B_{L}\right)
\end{array}\right|=\left|\begin{array}{cc}
T_{11} & T_{12} \\
T_{21} & T_{22}
\end{array}\right|\left|\begin{array}{c}
F_{R}+B_{R} \\
\gamma k_{s}\left(F_{R}+B_{R}\right)
\end{array}\right|
$$

From (2.28), we derive the governing equations for the determinations of propagation constants of guided and radiation modes for TE polarization [40-41]:

$$
f(\beta)=T_{21}+\gamma\left(k_{s} T_{22}+k_{c} T_{11}\right)+\gamma^{2} k_{c} k_{s} T_{12}=0
$$

For TM polarization, we obtain [40-41]

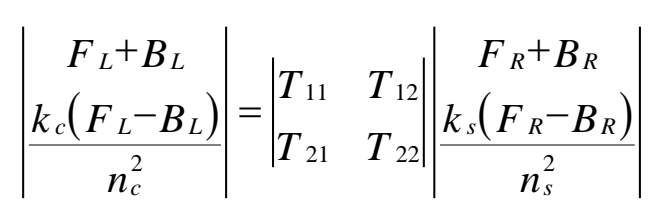

Instituting (2.26) and (2.27) into (2.30) [40-41]

$$
\left|\begin{array}{c}
F_{L}+B_{L} \\
\frac{-\gamma k_{c}\left(F_{L}+B_{L}\right)}{n_{c}^{2}}
\end{array}\right|=\left|\begin{array}{cc}
T_{11} & T_{12} \\
T_{21} & T_{22}
\end{array}\right|\left|\begin{array}{c}
F_{R}+B_{R} \\
\gamma k_{s}\left(F_{R}+B_{R}\right) \\
n_{s}^{2}
\end{array}\right|
$$

we further get the governing equation for TM polarization [40-41]

$$
f(\beta)=n_{c}^{2} n_{s}^{2} T_{21}+\gamma\left(n_{c}^{2} T_{22} k_{s}+n_{s}^{2} T_{11} k_{c}\right)+\gamma^{2} T_{12} k_{c} k_{s}=0
$$

Note that as $\gamma$ gradually increases from 0 to 1 , the currently obtained effective index $\left(N_{\text {eff }}\right)$ will work as the new initial guess for the next-step Newton iteration corresponding to new $\gamma$, just like Figure 2.3 shows. 
In conventional STM the mode spectrum depends on the position of artificial boundary a lot. It is recently reported that the artificial boundaries should be exactly placed at the interfaces between the claddings and multi-layer stacks [40].

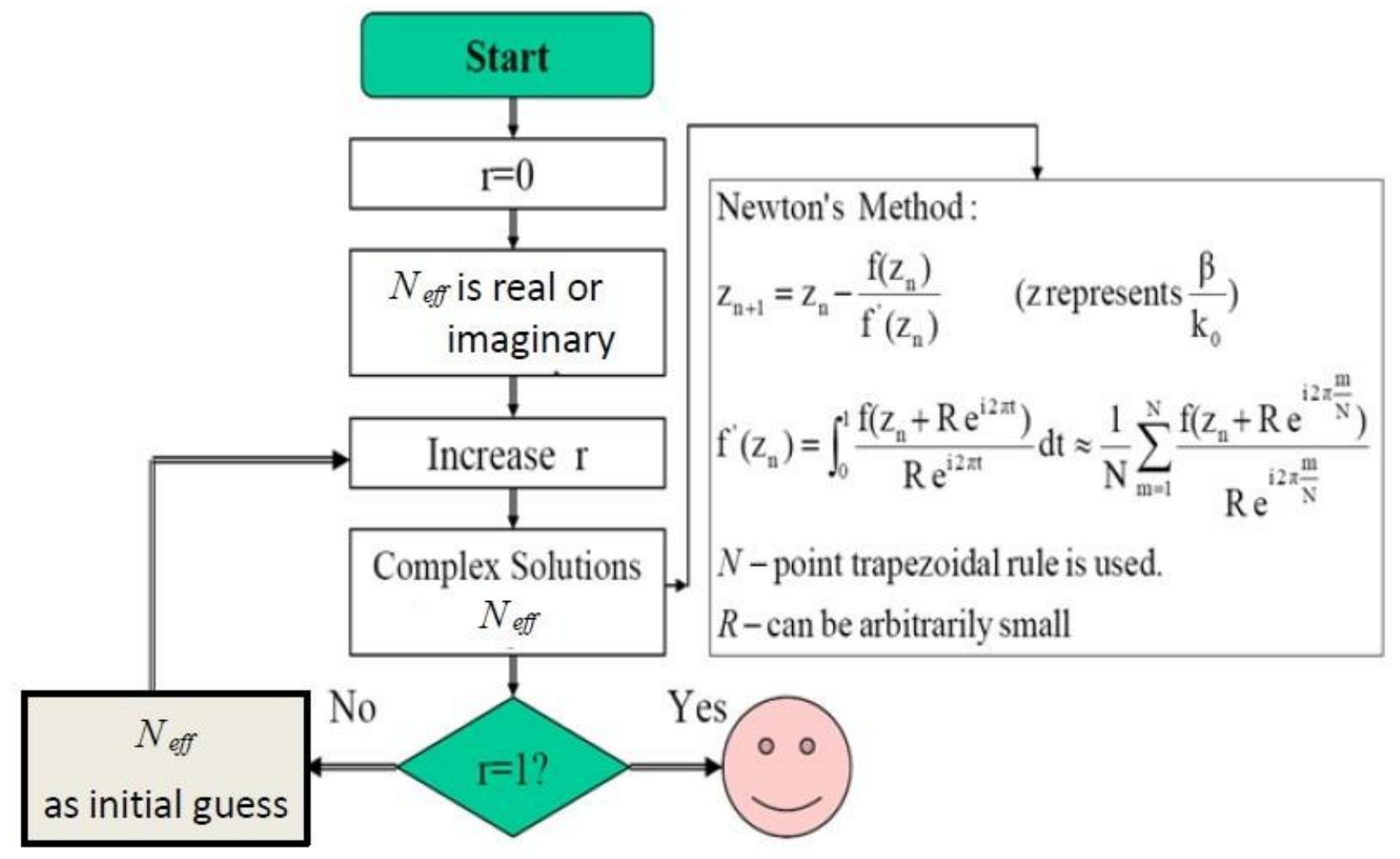

Figure 2.3. Methodology of Conventional STM

\subsection{Perfect Matching Layers as Numerical Boundary Condition}

\subsection{Discretized Box Modes}

We consider the open waveguide in Figure 2.4(a) and close waveguide in Figure 2.4(b). Through enclosing the open waveguide with Perfect Reflection Boundary (PRB), the continuous radiation waves will be discretized into box modes. If the 
computation window is large enough, the electric field for TE guided wave or the magnetic field for TM guided wave can be regarded as decayed to zero at the boundaries. However the refection at the closed boundary for radiating wave will affect the simulation results.

For the symmetric three-layer slab waveguide, its solutions of the governing equation (2.7) for $E_{y}$ in TE polarization case is (2.33) (for even modes) and (2.34) (for odd modes) [10].

$$
\begin{aligned}
& E_{y}=\left\{\begin{array}{cc}
\frac{\sin \left[k_{x, \text { clad }}(x+L)\right]}{\sin \left[k_{x, \text { clad }}(-S+L)\right]} & -L<x<-S \\
\frac{\cos \left(k_{x, \text { core }} x\right)}{\cos \left(k_{x, \text { core }} S\right)} & |x|<S \\
\frac{\sin \left[k_{x, \text { clad }}(x-L)\right]}{\sin \left[k_{x, \text { clad }}(S-L)\right]} & S<x<L
\end{array}\right. \\
& E_{y}=\left\{\begin{array}{cc}
-\frac{\sin \left[k_{x, \text { clad }}(x+L)\right]}{\sin \left[k_{x, \text { clad }}(-S+L)\right]} & -L<x<-S \\
\frac{\cos \left(k_{x, \text { core }} x\right)}{\cos \left(k_{x, \text { core }} S\right)} & |x|<S \\
\frac{\sin \left[k_{x, \text { clad }}(x-L)\right]}{\sin \left[k_{x, \text { clad }}(S-L)\right]} & S<x<L
\end{array}\right.
\end{aligned}
$$




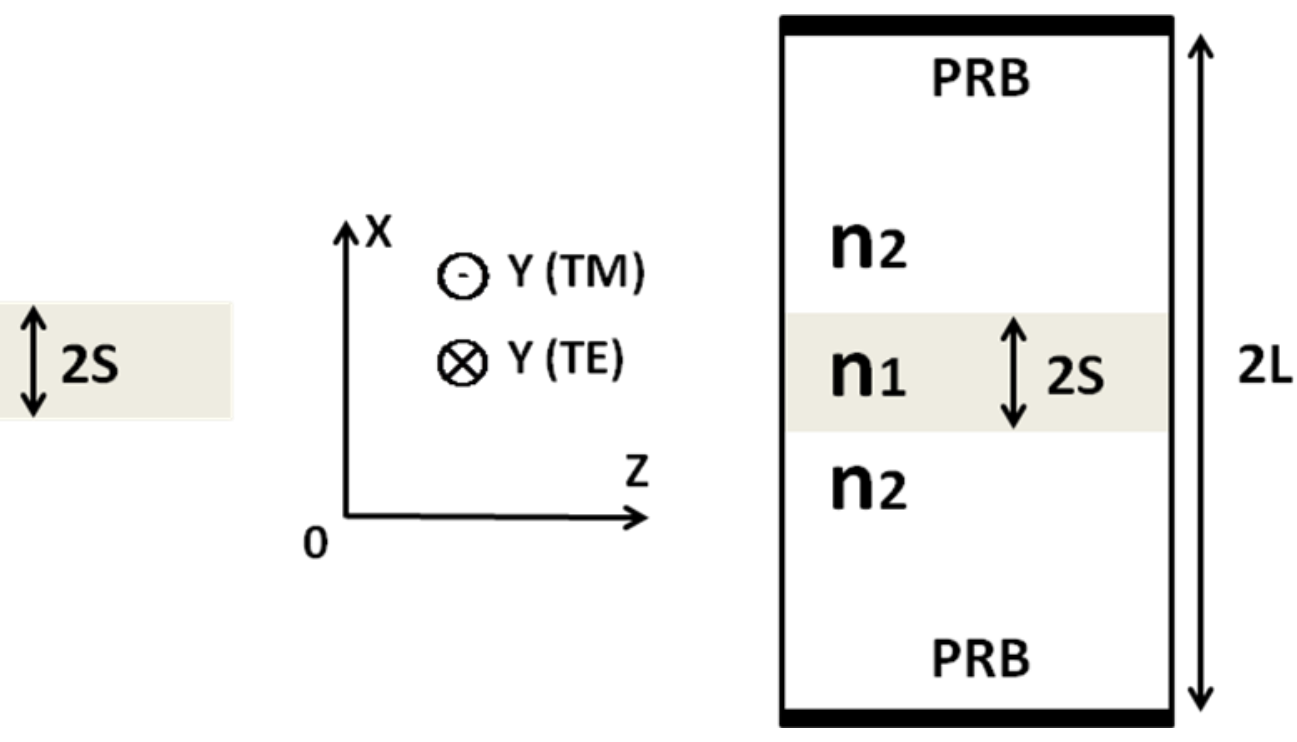

(a)

(b)

Figure 2.4. (a) 2-D open waveguide (b) 2-D close waveguide

where

$$
\begin{gathered}
k_{x, \text { clad }}=k_{0} \sqrt{n_{2}^{2}-N_{\text {eff }}^{2}} \\
k_{x, \text { core }}=k_{0} \sqrt{n_{1}^{2}-N_{\text {eff }}^{2}} \\
N_{\text {eff }}=\frac{\beta}{k_{0}}
\end{gathered}
$$

According to the continuity of $H_{x}$ at the two interfaces, we obtain the characteristic equation for even TE modes [10]

$$
-k_{x, \text { core }} \tan \left(k_{x, \text { core }} S\right)=k_{x, \text { clad }} \cot \left[k_{x, \text { clad }}(S-L)\right]
$$

and that for odd TM modes [10]

$$
-k_{x, \text { core }} \cot \left(k_{x, \text { core }} S\right)=k_{x, \text { clad }} \cot \left[k_{x, \text { clad }}(S-L)\right]
$$

The solutions for governing equation of $H_{y}$ in TM polarization case are (2.40) (for TM even modes) and (2.41) (for TM odd modes) [10]. 


$$
H_{y}=\left\{\begin{array}{cc}
\frac{\sin \left[k_{x, \text { clad }}(x+L)\right]}{\sin \left[k_{x, \text { clad }}(-S+L)\right]} & -L<x<-S \\
\frac{\cos \left(k_{x, \text { core }} x\right)}{\cos \left(k_{x, \text { core }} S\right)} & |x|<S \\
\frac{\sin \left[k_{x, \text { clad }}(x-L)\right]}{\sin \left[k_{x, \text { clad }}(S-L)\right]} & S<x<L
\end{array}\right\}
$$

According to the continuity of $E_{x}$ at the two interfaces, we obtain the characteristic equation for TM even mode [10]

$$
\frac{-k_{x, \text { core }} \tan \left(k_{x, \text { core }} S\right)}{n_{1}^{2}}=\frac{k_{x, \text { clad }} \cot \left[k_{x, \text { clad }}(S-L)\right]}{n_{2}^{2}}
$$

and that for TM odd modes [10]

$$
\frac{-k_{x, \text { core }} \cot \left(k_{x, \text { core }} S\right)}{n_{1}^{2}}=\frac{k_{x, \text { clad }} \cot \left[k_{x, \text { clad }}(S-L)\right]}{n_{2}^{2}}
$$

The solutions of (2.38), (2.39), (2.42) and (2.43) can be real, imaginary or complex.

Consequently the box modes contain the guided, evanescent and complex modes.

Figure 2.5 shows the mode spectrum in TE and TM polarization cases of the slab waveguide like Figure 2.4(b). The wavelength is $1.55 \mu \mathrm{m}$. When the box size is large enough, the guided mode spectrum will be independent on the box size, while the radiation mode spacing will turn smaller. 


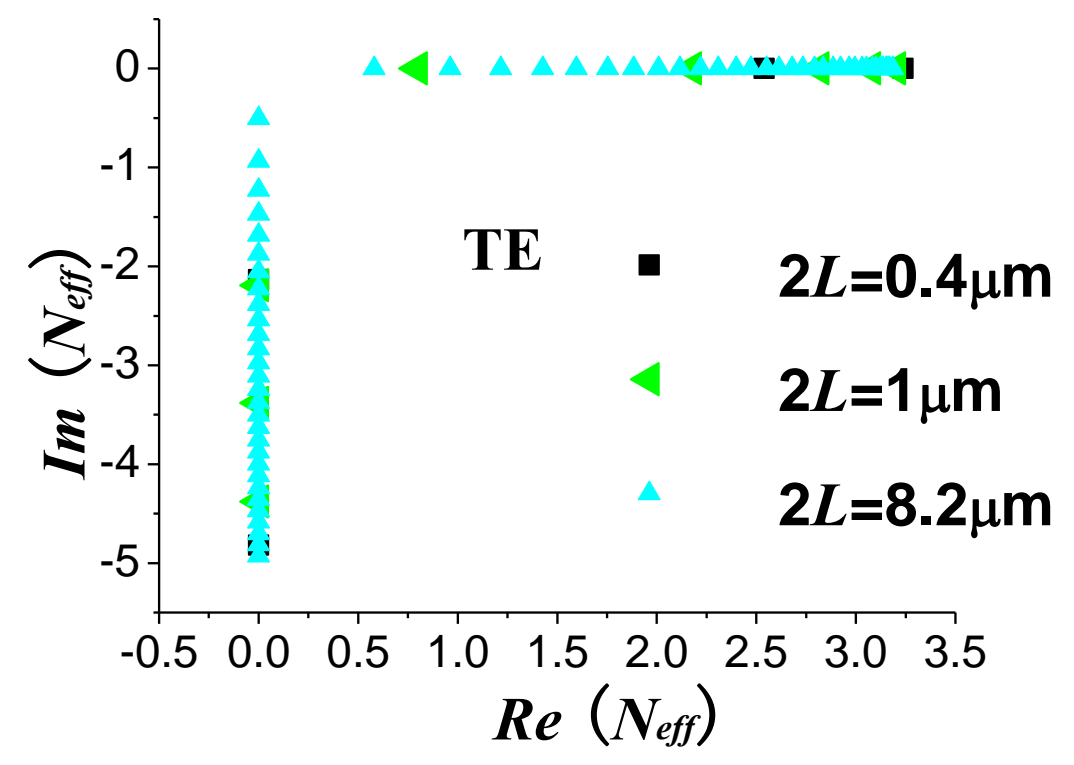

(a)

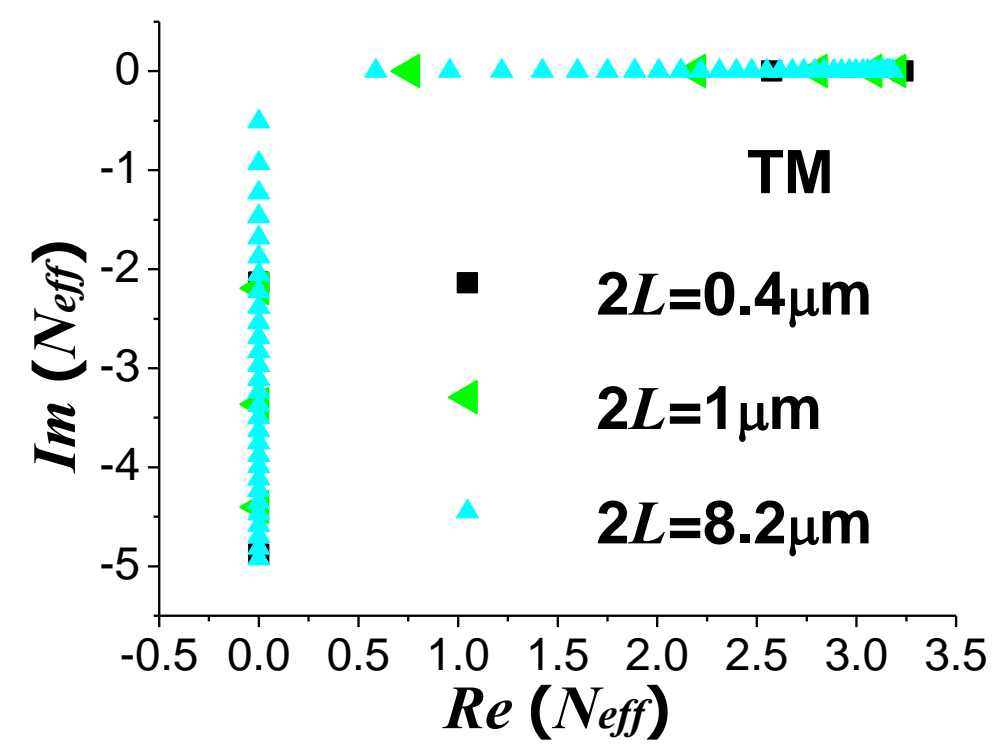

(b)

Figure 2.5. Mode spectrums of the closed symmetric slab waveguide in Figure 2.4(b) where $\mathrm{n} 1=3.3, \mathrm{n} 2=3.17,2 \mathrm{~S}=0.2 \mathrm{~nm}$ for (a)TE polarization $\quad$ (b) TM polarizations

\subsection{Modified 2-D Waveguide Governing Equation}


In order to reduce the reflection of radiating wave from PRB, we can place the absorbing Perfect Matching Layer (PML) adjacent to PRB to suppress inherent reflection [42-43]. The stretching coordinates of PML layer are complex number with minus imaginary parts. Accordingly the transversely outgoing field in PML region will be effectively attenuated. As a result appropriate PML parameters will make the waveguide region work as if in open waveguide. And the whole mode spectrum will be discretized into orthogonal and normalizable guided and complex modes [44]. Figure 2.6 shows a slab waveguide terminated with both PML and PRB on each side.

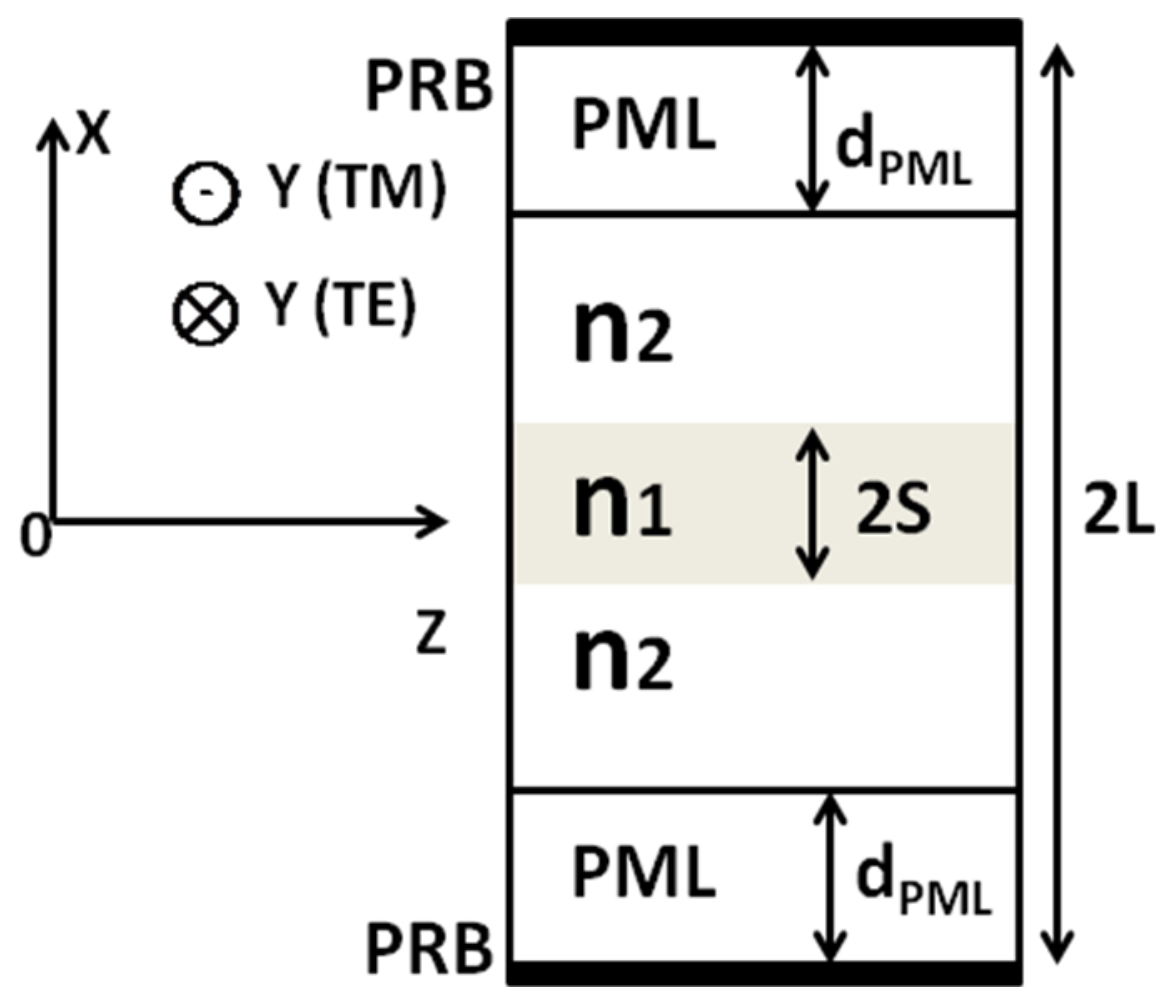

Figure 2.6. A 2-D slab waveguide enclosed with PML and PRB

As a non-physical fictitious medium applied to match the physical domain, the PML index is usually set to be equal with that of the adjacent cladding layer. 
Assuming PML media is anisotropic, the Maxwell's equation is modified to be [29-30]:

$$
\left\{\begin{array}{c}
\nabla_{t} \times \vec{E}-j \beta \vec{E}=-j \omega \mu_{0}|\Lambda| \vec{H} \\
\nabla_{t} \times \vec{H}-j \beta \vec{H}=j \omega \varepsilon|\Lambda| \vec{E}
\end{array}\right.
$$

where $|\Lambda|$ is defined as [29-30]

$$
|\Lambda|=\left|\begin{array}{ccc}
\alpha_{y} / \alpha_{x} & 0 & 0 \\
0 & \alpha_{x} / \alpha_{y} & 0 \\
0 & 0 & \alpha_{x} \alpha_{y}
\end{array}\right|
$$

Here $\alpha_{y}, \alpha_{x}$ and $\alpha_{z}$ are defined as the stretching factor along $\hat{Y}, \hat{X}$ and $\hat{Z}$. The waveguide lengths along $\hat{Y}$ and $\hat{Z}$ are infinite, and therefore $\alpha_{y}$ and $\alpha_{z}$ should be 1 . And $\alpha_{x}$ is defined as [29-30]

$$
\alpha_{x}=\kappa_{x 0}-j \frac{\sigma_{x 0}}{\omega_{\varepsilon_{0} n_{P M L}^{2}}}\left(\frac{\rho}{d_{P M L}}\right)^{2}
$$

where $\kappa_{x 0}$ is related to the evanescent modes, and $\sigma_{x 0}$ is the parameter for the attenuation of the travelling wave. $d_{P M L}$ is the thickness of PML layer, and $n_{P M L}$ is its index. $\rho$ is the distance between the calculated position and the starting point of PML, as shown in Figure 2.7. With the index of PML complex, the coordinates in PML is therefore complex [29-30].

$$
\tilde{x}=\int_{0}^{x} \alpha_{x}\left(x^{\prime}\right) d x^{\prime}
$$

Consequently the stretching derivation in PML region is [29-30]

$$
\frac{d}{d \tilde{x}}=\frac{1}{\alpha_{x}} \frac{d}{d x}
$$

Sometimes we make $\kappa_{x 0}$ with parabolic growth, in order to reduce dPML [29-30] 


$$
\kappa_{x 0}=1+\left(\frac{\rho}{d_{P M L}}\right)^{2}
$$

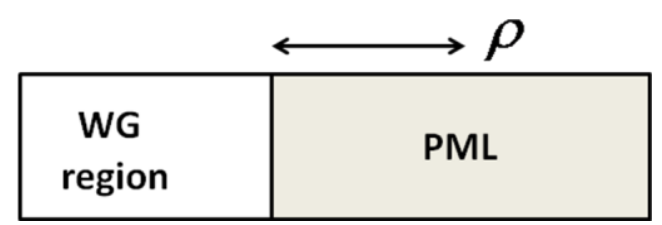

Figure 2.7. Implement of PML

With such coordinate stretching factors, the governing equation is modified for TE polarization [29-30]

$$
\begin{gathered}
\frac{1}{\alpha_{x}} \frac{\partial}{\partial x}\left(\frac{1}{\alpha_{x}} \frac{\partial E_{y}}{\partial x}\right)+\frac{\partial^{2} E_{y}}{\partial^{2} z}+n(x)^{2} k_{0}^{2} E_{y}=0 \\
H_{z}=-\frac{1}{j \omega \mu_{0} \alpha_{x}} \frac{\partial E_{y}}{\partial x} \\
H_{x}=-\frac{1}{j \omega \mu_{0}} \frac{\partial E_{y}}{\partial z}
\end{gathered}
$$

with $\frac{\partial E_{y}}{\partial x}=-j \kappa_{x} E_{y} \quad, \quad \frac{\partial E_{y}}{\partial z}=-j \beta E_{y}$. The dispersion relationship in TE polarization case is

$$
\left(\frac{\kappa_{x}}{\alpha_{x}}\right)^{2}+\beta^{2}=n^{2} k_{0}^{2}
$$

The modified governing equation in TM polarization case can be expressed as [29-30]

$$
\frac{1}{\alpha_{x}} \frac{1}{\partial x}\left(\frac{1}{\alpha_{x}} \frac{1}{n^{2}} \frac{\partial H_{y}}{\partial x}\right)+\frac{1}{\partial z}\left(\frac{1}{n^{2}} \frac{\partial H_{y}}{\partial z}\right)+k_{0}^{2} H_{y}=0
$$

With $\frac{\partial H_{y}}{\partial x}=-j \kappa_{x} H_{y}, \frac{\partial H_{y}}{\partial z}=-j \beta H_{y}$, the dispersion relationship in polarization TM case is

$$
\left(\frac{\kappa_{x}}{\alpha_{x}}\right)^{2}+\beta^{2}=n^{2} k_{0}^{2}
$$


The implement of PML should guarantee that there are no inherent reflections at the interface of waveguide region and PML layer, and the interfaces of different PML layers (Figure 2.8).

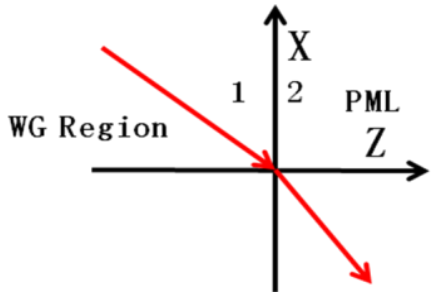

(a)

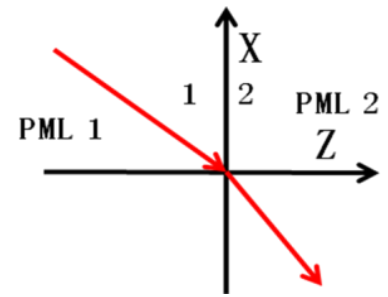

(b)

Figure 2.8. (a) The interface of waveguide region and PML (b) The interface of different PML layers.

Assuming $\mathrm{T}$ and $\mathrm{R}$ are transmission and reflection at the two kinds of interfaces in

Figure 2.8. The launched field

$$
\Phi_{1}=\exp \left(-j_{\kappa_{x 1}} x-j \beta Z\right)+\exp \left(j_{\kappa_{x 1}} x-j \beta Z\right) R
$$

The transmitted field at the interfaces

$$
\Phi_{2}=\exp \left(-j \kappa_{x 2} x-j \beta Z\right) T
$$

At the position of $(\mathrm{x}, \mathrm{z})=(0,0)$, according to the consistency of $E_{y}\left(H_{y}\right)$ in TE (TM) polarization case, we have

$$
1+R=T
$$

At the interfaces of PML layers, $\alpha_{x 1}=\alpha_{x 2}$; At the starting position of PML, $\alpha_{x}=1$. According to the continuity of $H_{x}\left(E_{x}\right)$ in TE (TM) polarization case in passive media, we have

$$
1-R=T
$$


Substituting (2.59) into (2.58), we have $R=0, T=1$. Consequently, regardless of the launching angle, polarizations and frequencies, there are no reflections at the interfaces of PML and waveguide region, and those of PML layers .

The reflection coefficient $R_{P M L}$ is a critical parameter determining the accuracy of PML-based waveguide model. In addition, $R_{P M L}$ is independent of $\kappa_{x 0}$. From (2.60) and (2.61) [29-30]

$$
\begin{gathered}
R=\left|\exp \left(-2 j \kappa_{0} \tilde{d}_{P M L}\right)\right| \\
\tilde{d}_{P M L}=\int_{0}^{d_{P M L}} \alpha\left(x^{\prime}\right) d x^{\prime}=d_{P M L}+\frac{d_{P M L}}{3}-j \frac{\sigma_{x 0}}{\omega_{\mathcal{E}_{0} n_{P M L}^{2}}^{2}} \frac{d_{P M L}}{3}
\end{gathered}
$$

We get the expressions for reflection coefficient as

$$
R_{P M L}=\exp \left(-\frac{2 \sigma_{x 0}}{n_{P M L} \sqrt{\varepsilon_{0} / \mu_{0}}} \int_{0}^{d_{P M L}}\left(\frac{\rho}{d_{P M L}}\right)^{2} d \rho\right)
$$

Thus the stretching factor is [29-30]

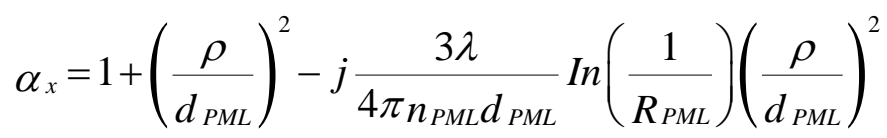




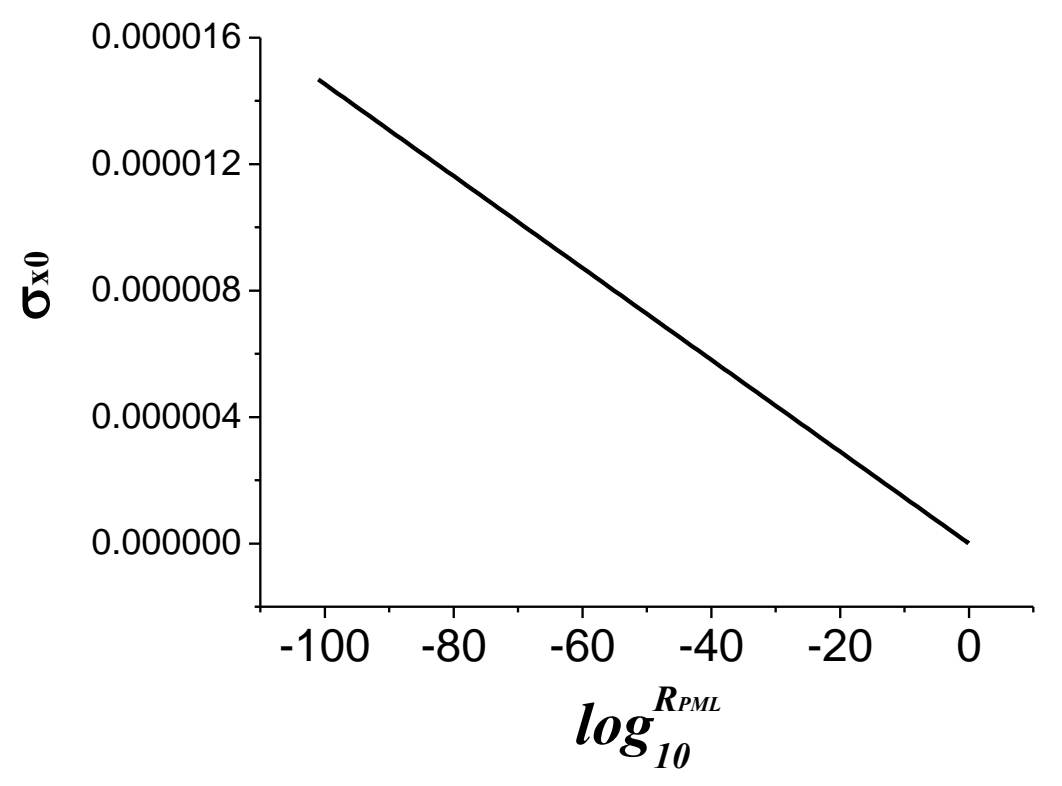

Figure 2.9. The evolution of $\sigma_{x 0}$ with $R_{P M L}$ when $\mathrm{n} 2=3.17 . \mathrm{d}_{\mathrm{PML}}=5 \mu \mathrm{m} . \quad \lambda=1.55 \mu \mathrm{m}$ in Figure 2.6(b)

\subsection{Hollow Waveguide}

Figure 2.10 shows a hollow waveguide enclosed with PML and PRB. 


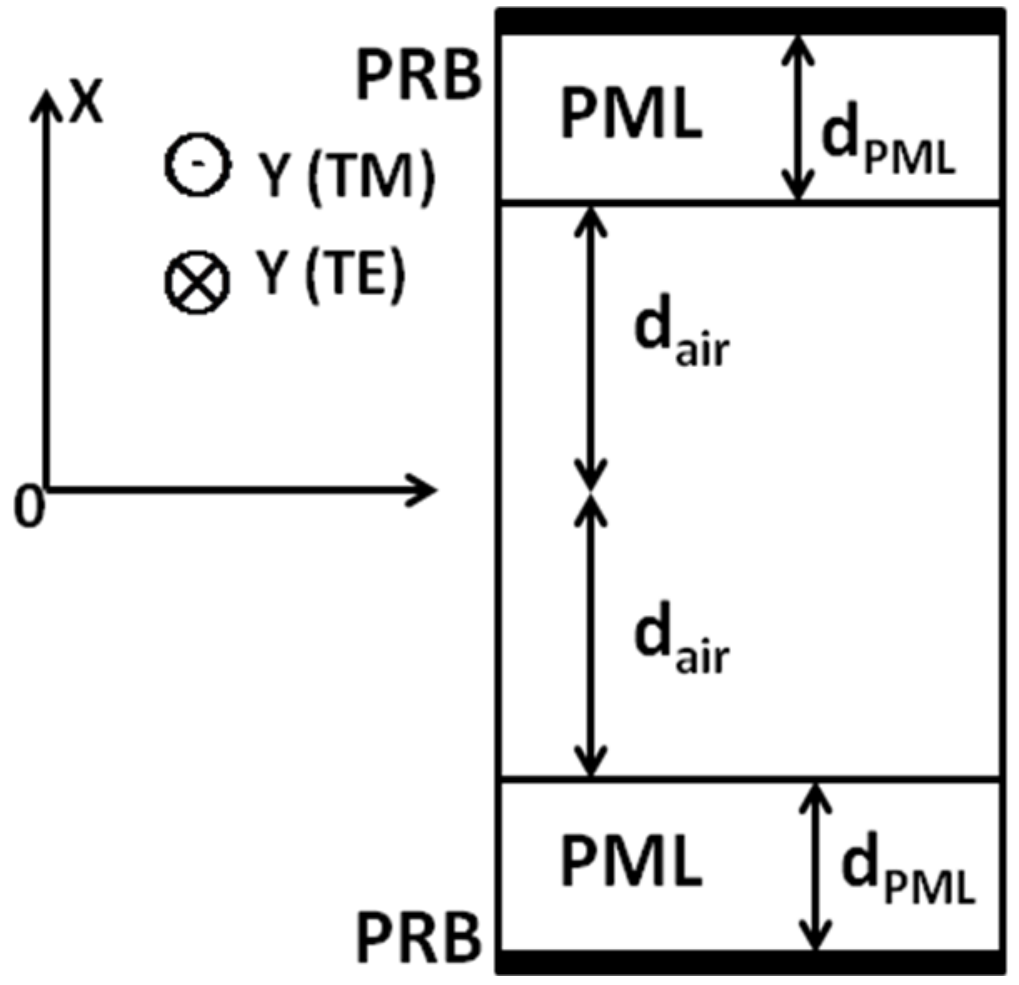

Figure 2.10. The schematic of a 2-D hollow waveguide enclosed with PML and PRB on each side

According to the governing equation of $E_{y}\left(H_{y}\right)$ in TE (TM) polarization, and the boundary condition in hollow waveguide for TE polarization

$$
\begin{aligned}
& E_{y}\left(d_{\text {air }}+d_{P M L}\right)=0 \\
& E_{y}\left(-d_{\text {air }}-d_{P M L}\right)=0
\end{aligned}
$$

And the boundary condition for TM polarization

$$
\begin{aligned}
& H_{y}\left(d_{\text {air }}+d_{P M L}\right)=0 \\
& H_{y}\left(-d_{\text {air }}-d_{P M L}\right)=0
\end{aligned}
$$

We get the analytical solutions of field pattern in TE case [52]

$$
\left\{\begin{array}{c}
E_{y}=\sin \left(\kappa_{x, n} \tilde{x}(x)\right) \\
H_{x}=-\frac{\alpha(x) \beta_{n}}{\omega \mu_{0}} \sin \left(\kappa_{x, n} \tilde{x}(x)\right) \\
H_{z}=-\frac{\kappa_{x, n}}{j \omega \mu_{0}} \cos \left(\kappa_{x, n} \tilde{x}(x)\right)
\end{array}\right.
$$


The analytical solutions for modal field pattern for TM polarization is [52]

$$
\left\{\begin{array}{c}
H_{y}=\cos \left(\kappa_{x, n} \tilde{x}(x)\right) \\
E_{x}=-\frac{\alpha(x) \beta_{n}}{\omega_{\varepsilon_{0}}} \cos \left(\kappa_{x, n} \tilde{x}(x)\right) \\
E_{z}=-\frac{\kappa_{x, n}}{j \omega_{\varepsilon_{0}}} \sin \left(\kappa_{x, n} \tilde{x}(x)\right)
\end{array}\right.
$$

where $\mathrm{n}=0,1,2, \cdots \cdots, \kappa_{x, n}$ is the transverse wave vector for the $n^{\text {th }}$ mode

For the even modes [52]

$$
\kappa_{x, n}\left(d_{\text {air }}+\tilde{d}_{P M L}\right)=n \pi
$$

For the odd modes [52]

$$
\kappa_{x, n}\left(d_{\text {air }}+\tilde{d}_{P M L}\right)=(n+0.5) \pi
$$

The propagation constant for the $\mathrm{n}^{\text {th }}$ mode

$$
\beta_{n}=\sqrt{k_{0}^{2}-\kappa_{x, n}^{2}}
$$

The stretching coordinate $\tilde{x}$ is defined as

$$
\tilde{x}=\left\{\begin{array}{cl}
x-j \frac{\sigma_{x 0}}{\omega_{\varepsilon_{0}}} \frac{\left(x-d_{\text {air }}\right)^{2}}{3 d_{P M L}^{2}}, & x>d_{\text {air }} \\
x \quad, \quad|x|<d_{\text {air }} & \\
x-j \frac{\sigma_{x 0}}{\omega_{\mathcal{E}_{0}} \frac{\left(x+d_{\text {air }}\right)^{2}}{3 d_{P M L}^{2}}}, & x<-d_{\text {air }}
\end{array}\right.
$$

We can see that computation window size, dPML and $\frac{\sigma_{x 0}}{\omega_{\varepsilon_{0}}}$ will obviously affect the mode spectrum and the modal field patterns. Besides small enough $R_{P M L}$, a large enough computation window is necessary for the combination of complex modes to represent the continuous radiation wave well. With $\mathrm{dPML}=2 \mu \mathrm{m}$, Figure 2.11 compares the mode spectrums of hollow waveguides with the computation windows of $14 \mu \mathrm{m}$ 
$(4.2 \mu \mathrm{m})$ in case 1 (case 2$)$. It is shown that larger computation window promises smaller mode spacing for PML-implemented waveguide.

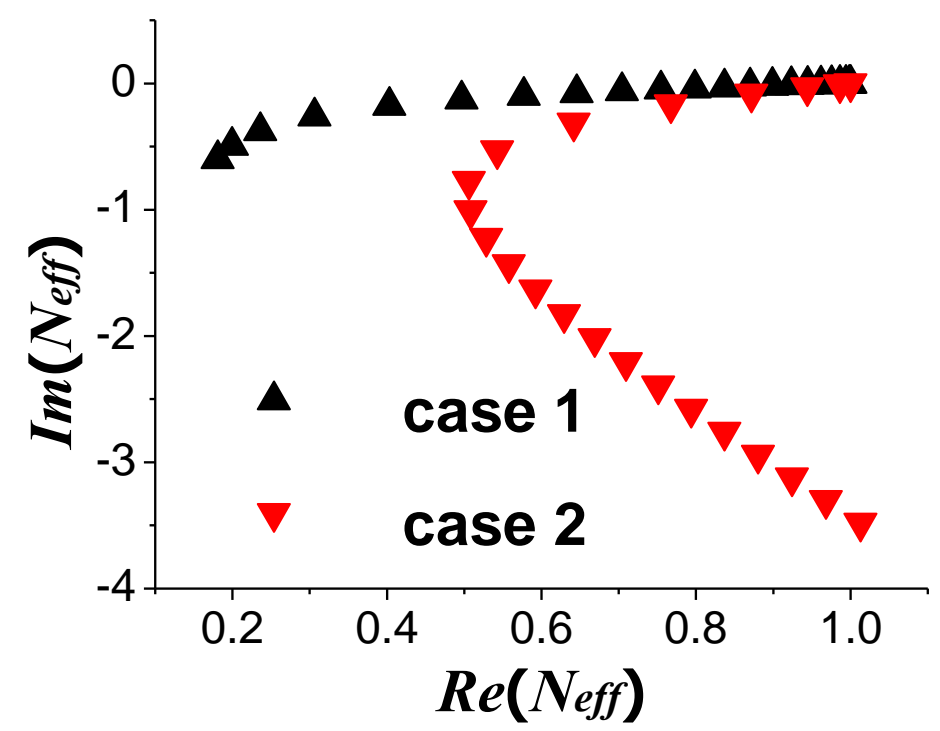

Figure 2.11. The mode spectrums of hollow waveguides with different computation windows. $R_{P M L}=1 \mathrm{e}-2 . \quad \lambda=1.55 \mu \mathrm{m}$.

From (2.62) and (2.67)-(2.69), $R_{P M L}$ has critical influence on the mode spectrum. Figure 2.12 shows the evolution of effective index $\left(N_{\text {eff }}\right)$ of the $10^{\text {th }}$ odd and even modes for the hollow waveguide with $R_{P M L}$. dair $=5 \mu \mathrm{m}, \mathrm{dPML}=2 \mu \mathrm{m}, \lambda$ $=1.55 \mu \mathrm{m}$. Considering the extreme case that,

$$
R_{P M L} \rightarrow 0
$$

for an arbitrary mode of the hollow waveguide, $\beta \rightarrow k_{0}$, which indicates that the modal field pattern behaves like plane wave in free space. 


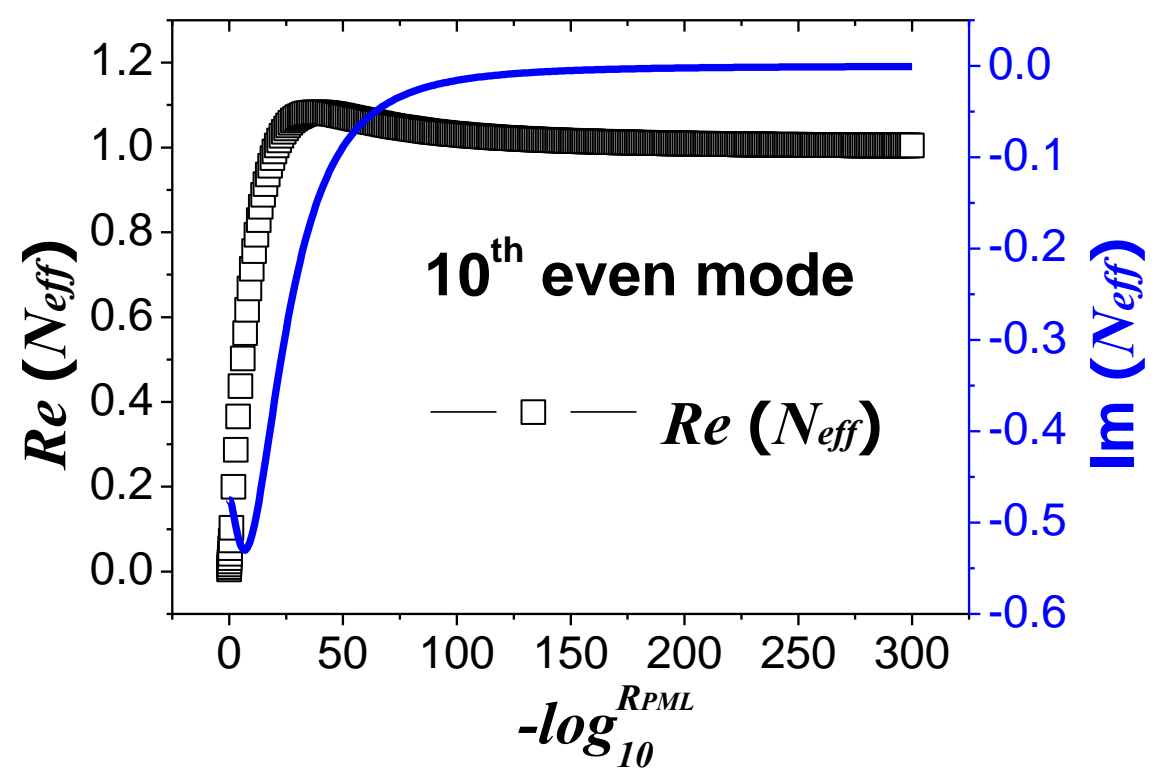

(a)

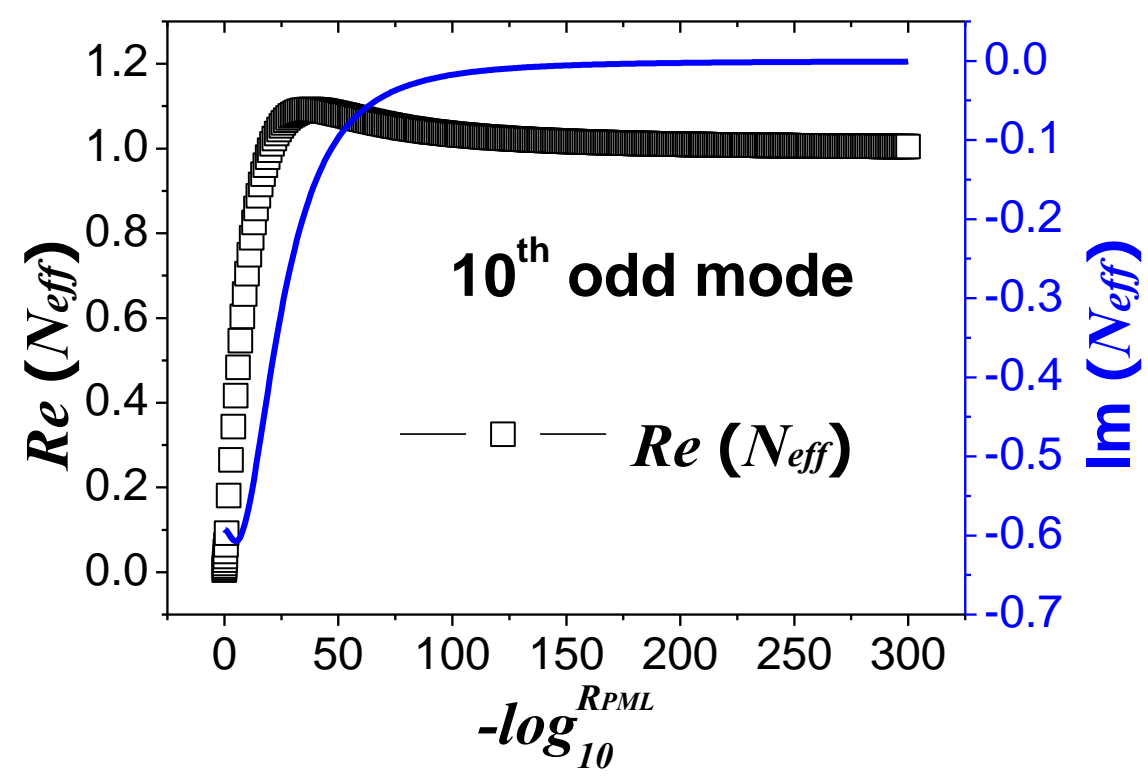

(b)

Figure 2.12. The evolutions of $N_{\text {eff }}$ of (a) the $10^{\text {th }}$ even mode and (b) the $10^{\text {th }}$ odd mode with $R_{P M L}$ 
With $\kappa_{x 0}$ as 1 , we study the influence of $R_{P M L}$ on mode spectrum in hollow waveguide with dair $=5 \mu \mathrm{m}, \mathrm{dPML}=2 \mu \mathrm{m}$ and $\lambda=1.55 \mu \mathrm{m}$ (Figure 2.13 ).

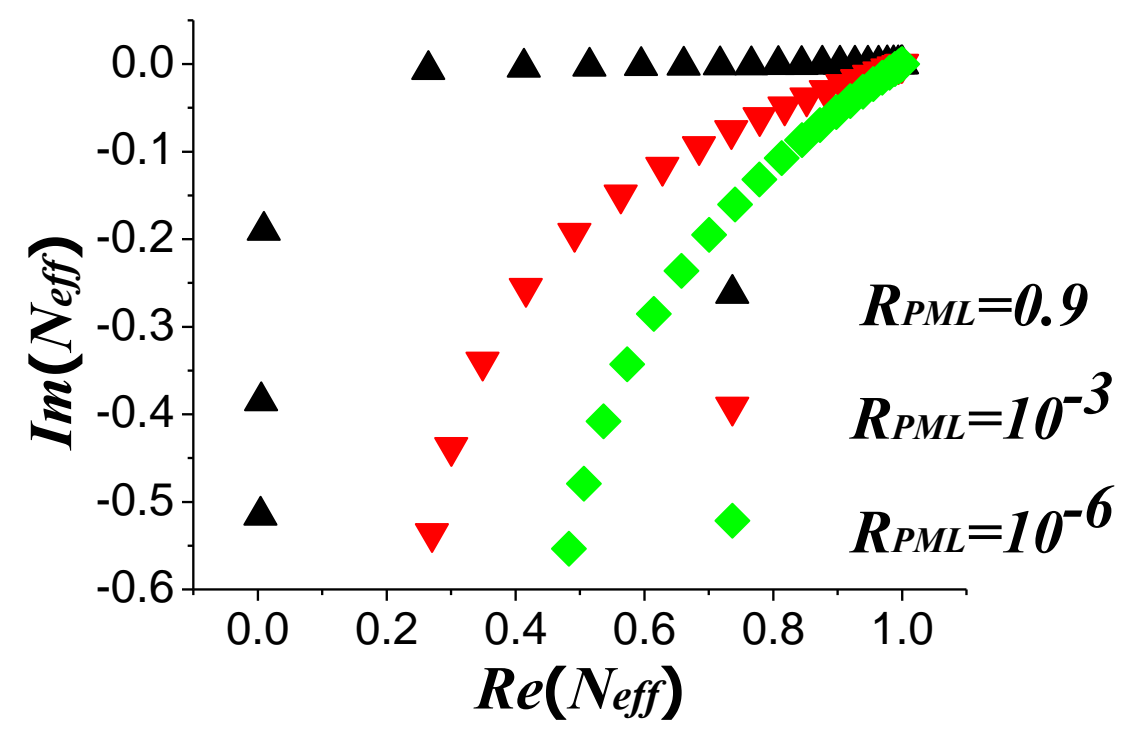

Figure 2.13. The impact of $R_{P M L}$ on mode spectrums in hollow waveguide

Figure 2.14 and Figure 2.15 describe the influence of $R_{P M L}$ on modal field patterns in TE and TM polarization cases, respectively, for the hollow waveguide structure mentioned above. 


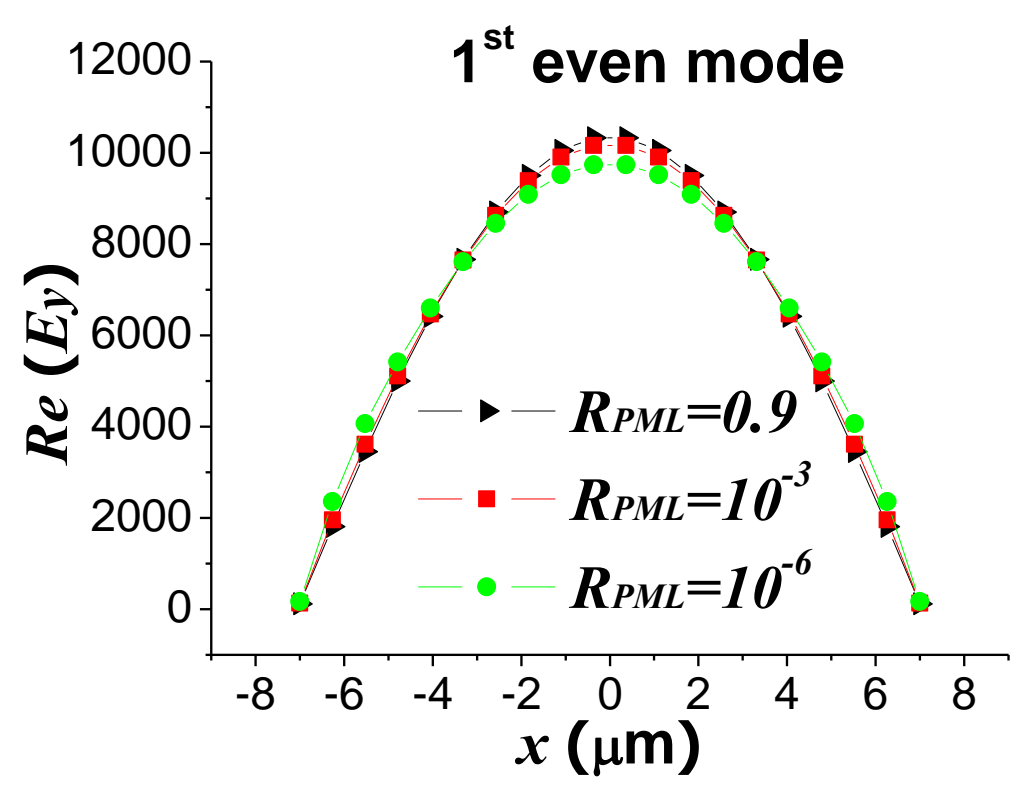

(a)

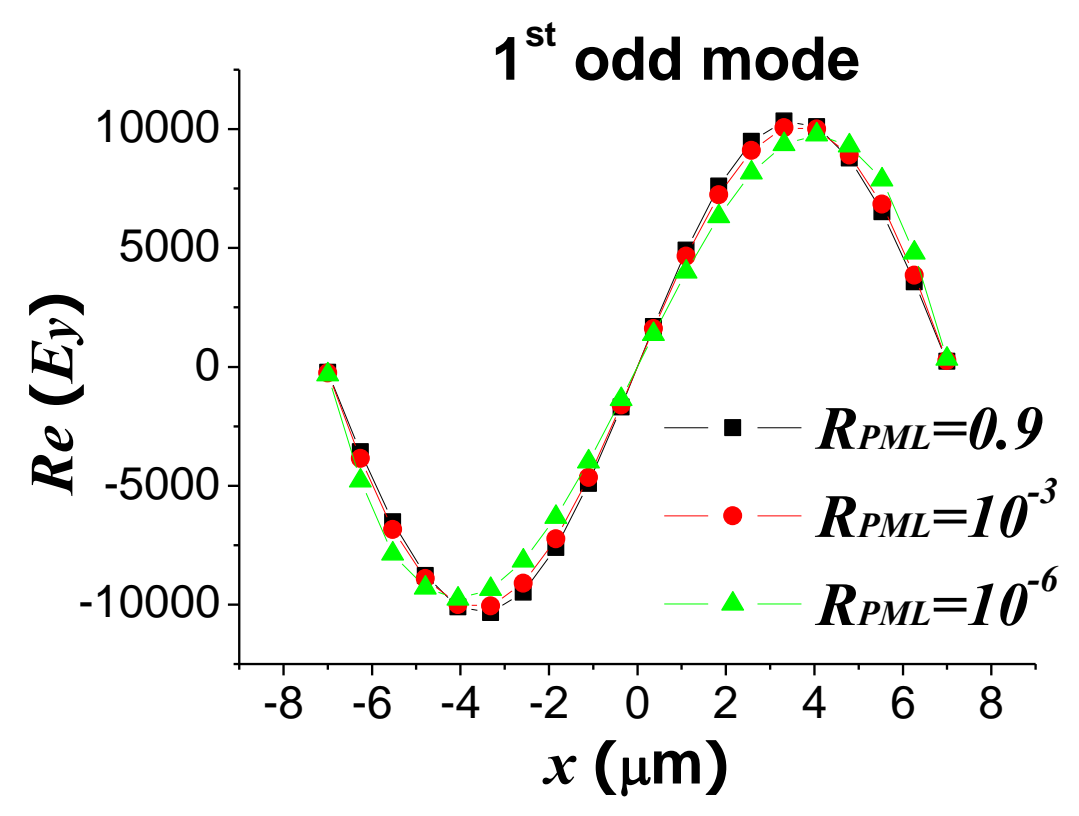

(b)

Figure 2.14. The influence of $R_{P M L}$ on modal electric field patterns of (a) $1^{\text {st }}$ even mode, and (b) $1^{\text {st }}$ odd mode in hollow waveguide for TE polarization. 


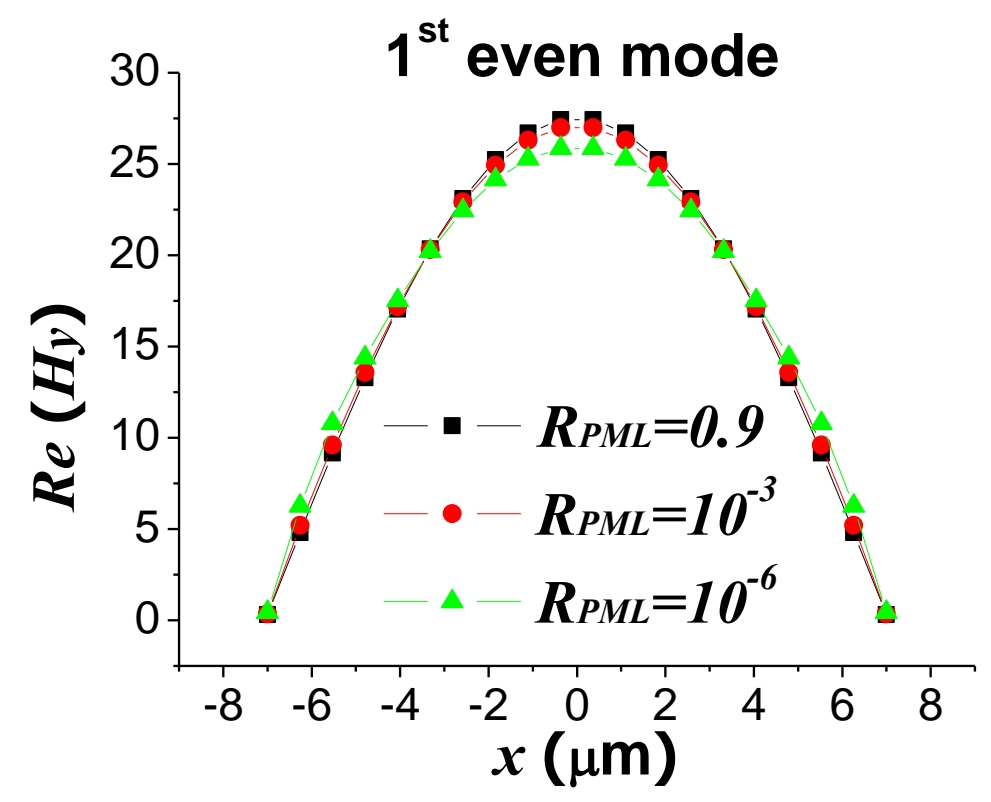

(a)

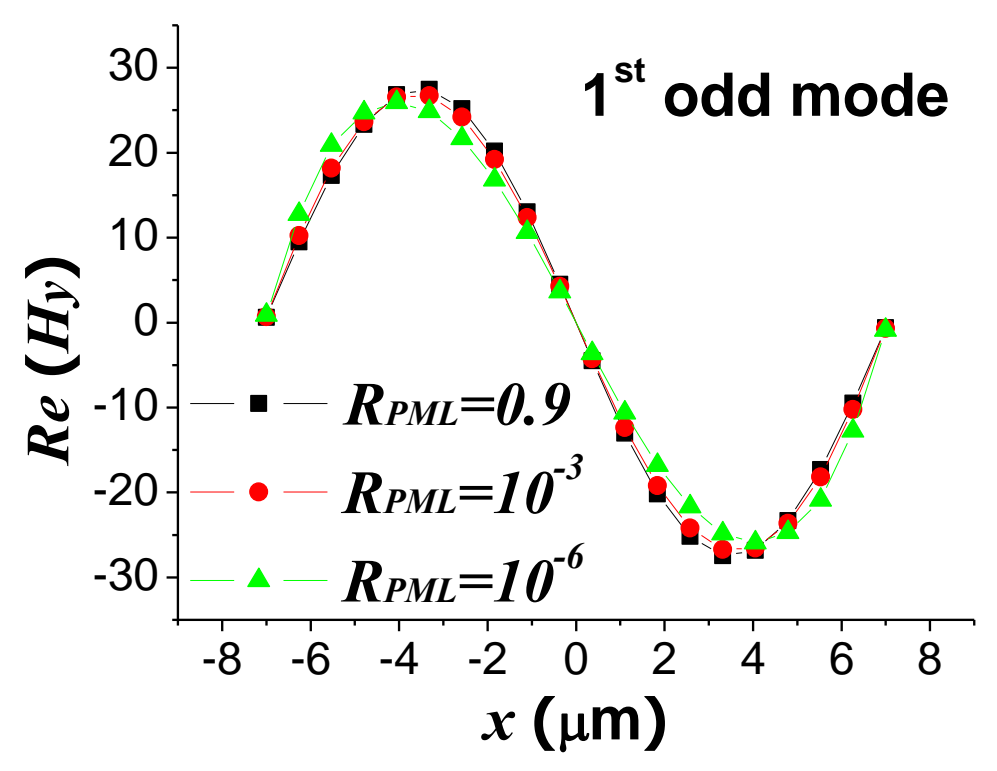

(b)

Figure 2.15. The influence of $R_{P M L}$ on modal magnetic field patterns of (a) $1^{\text {st }}$ even mode and (b) $1^{\text {st }}$ odd mode in hollow waveguide for TM polarization.

In order to study the orthogonality of analytical modal patterns in hollow waveguide, we consider the $\mathrm{m}^{\text {th }}$ and $\mathrm{n}^{\text {th }}$ TE modes of the free-space waveguide. 


$$
\int_{-d_{a i r}-d_{P M L}}^{d_{a i r}+d_{P M L}} E_{y, m} E_{y, n} d \tilde{x}=\int_{-d_{a i r}-d_{P M L}}^{d_{a i r}+d_{P M L}} \sin \left[\kappa_{x, m} \tilde{x}(x)\right] \sin \left[\kappa_{x, n} \tilde{x}(x)\right] d \tilde{x}
$$

with $d \tilde{x}=\alpha_{x} d x$, we further simplify (2.72) to be

$$
\int_{-d_{a i r}-d_{P M L}}^{d_{\text {air }}+d_{P M L}} E_{y, m} E_{y, n} d \tilde{x}=\int_{-d_{\text {air }}-\tilde{d}_{P M L}}^{d_{\text {air }}+\tilde{d}_{P M L}} \alpha_{x} \sin \left[\kappa_{x, m} \tilde{x}(x)\right] \sin \left[\kappa_{x, n} \tilde{x}(x)\right] d x
$$

When $\mathrm{m} \neq \mathrm{n}$, we have

$$
\int_{-d_{\text {air }}-d_{P M L}}^{d_{\text {air }}+d_{P M L}} E_{y, m} E_{y, n} d \tilde{x}=0
$$

In TM polarization case,

$$
\int_{-d_{a i r}-d_{P M L}}^{d_{a i r}+d_{P M L}} H_{y, m} H_{y, n} d \tilde{x}=\int_{-d_{a i r}-\tilde{d}_{P M L}}^{d_{a i r}+\tilde{d}_{P M L}} \alpha_{x} \cos \left[\kappa_{x, m} \tilde{x}(x)\right] \cos \left[\kappa_{x, n} \tilde{x}(x)\right] d x
$$

In the same way, if $m \neq n$, we have

$$
\int_{-d_{a i r}-d_{P M L}}^{d_{a i r}+d_{P M L}} H_{y, m} H_{y, n} d \tilde{x}=0
$$

Consequently the orthogonality of modal field patterns in hollow waveguide in both TE and TM polarization cases has been proved.

\subsection{High Order Finite Difference (HOFD) Scheme}

The mode calculation is the prerequisite for the modeling of wave propagation in photonics circuits in frequency-domain way. In the past, several numerical methods have been suggested to realize the mode solver, say the finite-element method [46], the method of lines [47-48] and the finite difference method [39]. Among them finite difference scheme is the most widely accepted due to its convenience and simplicity. 


\subsection{Formulations of HOFD Scheme}

The conception of graded index approximation is firstly suggested in the sense that the discontinuity of the dielectric medium will be matched through averaging the permitivity over meshes [49]. Recently Chiou develop the FD scheme by further applying the Douglas Scheme further and achieve the fourth order truncation error [39].

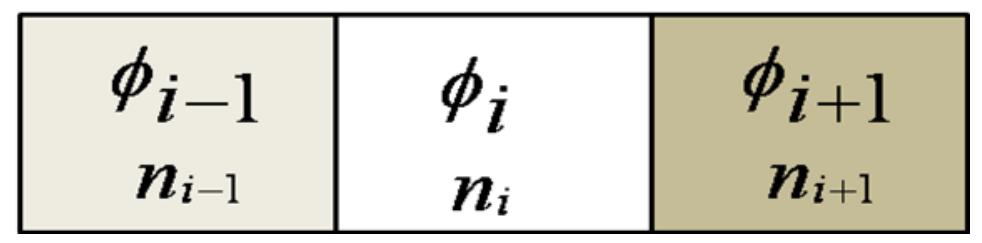

Figure 2.16. Three 1-D adjacent sampled points for HOFD

We consider the electric field (magnetic field) $\phi_{i}$ at an arbitrary sampled point in TE (TM) polarization case, and its two nearby fields are denoted as $\phi_{i-1}$ and $\phi_{i+1}$ (Figure 2.16). $\phi_{i}$ represents $E_{y}$ for TE case and does $H_{y}$ for TM case. Two arbitrary sampled points can be related by Taylor series and the continuity of $E_{y}$ $\left(H_{y}\right)$ at the boundary of two adjacent meshes. Thus $\phi_{i-1}$ and $\phi_{i+1}$ can be expressed about $\phi_{i}$ in the format of infinite series as [39]

$$
\begin{gathered}
\phi_{i-1}=e_{0} \phi_{i}+e_{1} \phi_{i}^{(1)}+e_{2} \phi_{i}^{(2)}+e_{3} \phi_{i}^{(3)}+e_{4} \phi_{i}^{(4)}+O\left(h^{5}\right) \\
\phi_{i+1}=f_{0} \phi_{i}+f_{1} \phi_{i}^{(1)}+f_{2} \phi_{i}^{(2)}+f_{3} \phi_{i}^{(3)}+f_{4} \phi_{i}^{(4)}+O\left(h^{5}\right)
\end{gathered}
$$

By omitting the high order terms in the infinite series above, and combining (2.77) and (2.78), we get the first and second order derivations of $\phi_{i}[39]$

$$
\phi_{i}^{(1)}=\frac{f_{2} \phi_{i-1}+\left(f_{0} e_{2}-e_{0} f_{2}\right) \phi_{i}-e_{2} \phi_{i+1}}{e_{1} f_{2}-f_{1} e_{2}}
$$




$$
\phi_{i}^{(2)}=\frac{f_{1} \phi_{i-1}+\left(f_{0} e_{1}-e_{0} f_{1}\right) \phi_{i}-e_{1} \phi_{i+1}}{e_{2} f_{1}-f_{2} e_{1}}
$$

We define the operators $D_{x}$ and $D_{x}^{2}$ as $D_{x} \phi_{i}=\phi_{i}^{(1)}$ and $D_{x}^{2} \phi_{i}=\phi_{i}^{(2)}$

By omitting the high order terms and combining (2.30) and (2.31) [39]

$$
\phi_{i}^{(2)}=\frac{f_{1} \phi_{i-1}+\left(f_{0} e_{1}-e_{0} f_{1}\right) \phi_{i}-e_{1} \phi_{i+1}}{e_{2} f_{1}-e_{1} f_{2}}+\frac{e_{1} f_{3}-e_{3} f_{1}}{e_{2} f_{1}-e_{1} f_{2}} \phi_{i}^{(3)}+\frac{e_{1} f_{4}-e_{4} f_{1}}{e_{2} f_{1}-e_{1} f_{2}} \phi
$$

By substituting (2.80), $\phi_{i}^{(3)}=D_{x} \phi_{i}^{(2)}$ and $\phi_{i}^{(4)}=D_{x}^{2} \phi_{i}^{(2)}$ into (2.81), we get [39]

$$
\phi_{i}^{(2)}=\frac{D_{x}^{2} \phi_{i}}{1+g_{1} D_{x}+g_{2} D_{x}^{2}}
$$

where

$$
\begin{gathered}
g_{1}=\frac{f_{3} e_{1}-e_{3} f_{1}}{e_{2} f_{1}-f_{2} e_{1}} \\
g_{2}=\frac{f_{4} e_{1}-e_{4} f_{1}}{e_{2} f_{1}-f_{2} e_{1}}
\end{gathered}
$$

Further we put (2.82) into the 2-D straight waveguide governing equation, we get [39]

$$
D_{x}^{2} \phi_{i}+\left(1+g_{1} D_{x}+g_{2} D_{x}^{2}\right) n^{2} k_{0}^{2} \phi_{i}=\left(1+g_{1} D_{x}+g_{2} D_{x}^{2}\right) \beta^{2} \phi_{i}
$$

For an arbitrary sampled point, the left and right side of the equation is linear superposition of $\phi_{i}, \phi_{i-1}$ and $\phi_{i+1}$. By combining each line corresponding to each sampled point into a square matrix, (2.86) can be denoted as

$$
\begin{gathered}
A \Phi=\beta^{2} C \Phi \\
\Phi=\left[\phi_{1}, \phi_{2}, \ldots \ldots, \phi_{i}, \ldots . . \phi_{N}\right]^{\prime}
\end{gathered}
$$

$\mathrm{N}$ is the number of sampled points. The eigenvalue equation (2.86) can be solved by Arnoldi iteration method[50].

Note that [39] 


$$
\begin{gathered}
D_{x} \phi_{1}=\frac{\left(f_{0} e_{2}-e_{0} f_{2}\right) \phi_{1}-e_{2} \phi_{2}}{e_{1} f_{2}-f_{1} e_{2}} \\
D_{x}^{2} \phi_{1}=\frac{\left(f_{0} e_{1}-e_{0} f_{1}\right) \phi_{1}-e_{1} \phi_{2}}{e_{2} f_{1}-f_{2} e_{1}} \\
D_{x} \phi_{N}=\frac{f_{2} \phi_{N-1}+\left(f_{0} e_{2}-e_{0} f_{2}\right) \phi_{N}}{e_{1} f_{2}-f_{1} e_{2}} \\
D_{x}^{2} \phi_{N}=\frac{f_{1} \phi_{N-1}+\left(f_{0} e_{1}-e_{0} f_{1}\right) \phi_{N}}{e_{2} f_{1}-f_{2} e_{1}}
\end{gathered}
$$

\subsection{Validation of HOFD Scheme in Slab Waveguide}

In order to validate HOFD scheme for 2-D slab waveguide, we consider a symmetric slab waveguide in Figure 2.4(b), with $\mathrm{n}_{1}=3.3, \mathrm{n}_{2}=3.17,2 \mathrm{~S}=2 \mu \mathrm{m}$, and $\lambda=1.55 \mu \mathrm{m}$. We calculate the guided modes of this structure, and validate the results with those from conventional STM. Table 2.1 and 2.2 show the validations of guided mode $N_{\text {eff }}$ in TE and TM polarizations, respectively. And Figure 2.16 and Figure 2.17 show the normalized guided mode patterns for TE and TM polarizations, respectively.

Table 1 The comparisons of guided mode $N_{\text {eff }}$ calculated from conventional STM and HOFD in slab waveguide (Figure 2.4(b)) with $\mathrm{n}_{1}=3.3, \mathrm{n}_{2}=3.17,2 \mathrm{~S}=2 \mu \mathrm{m}$, and $\lambda$ $=1.55 \mu \mathrm{m}$ for $\mathrm{TE}$ polarization

\begin{tabular}{|c|l|l|l|}
\hline Mode & TE0 & TE1 & TE2 \\
\hline FD & 3.2860 & 3.2451 & 3.1856 \\
\hline STM & 3.2860 & 3.2451 & 3.1856 \\
\hline
\end{tabular}




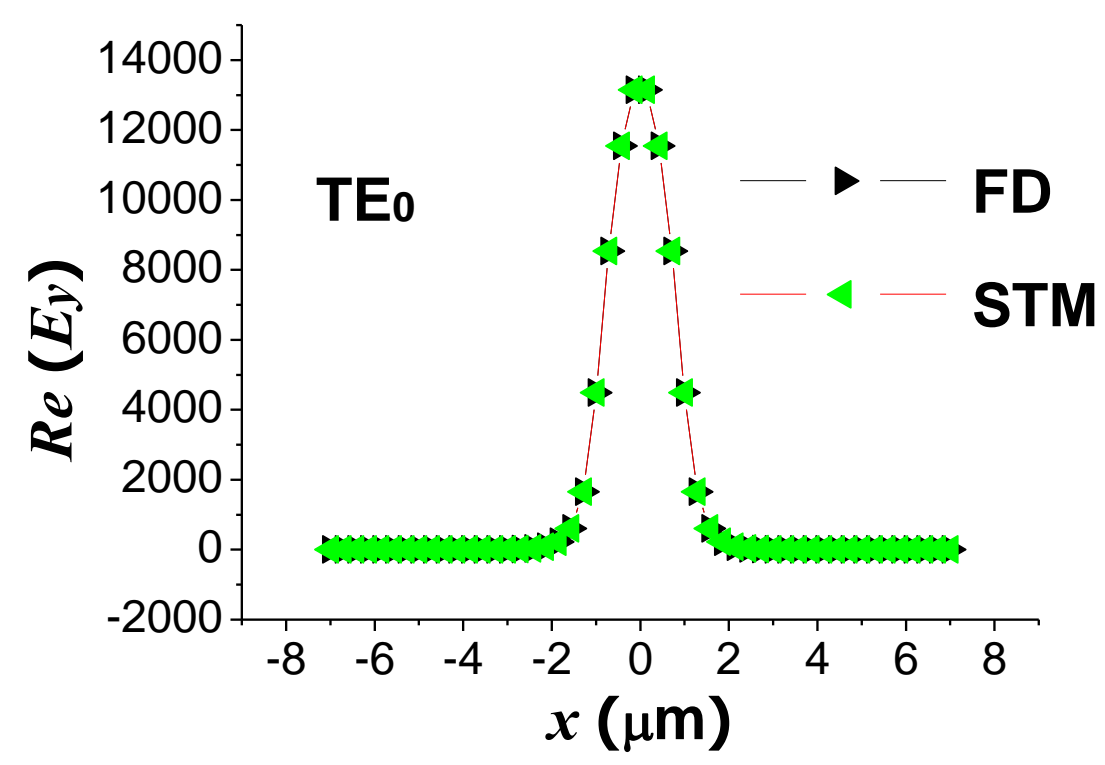

(a)

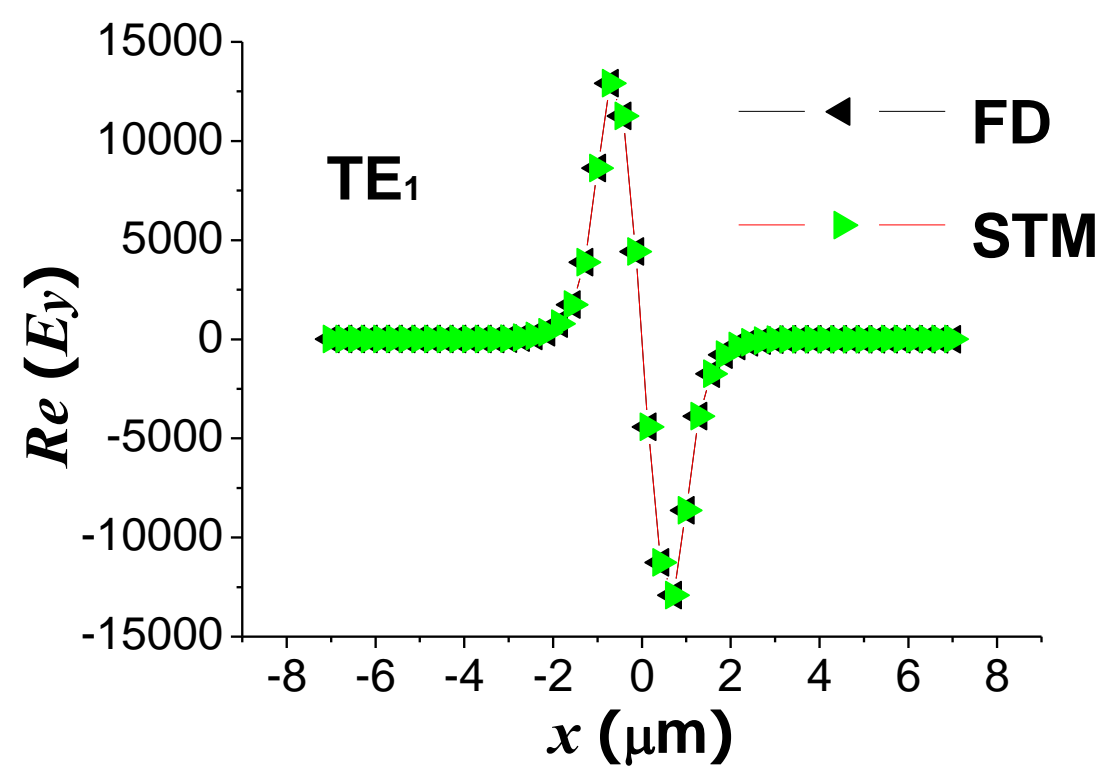

(b) 


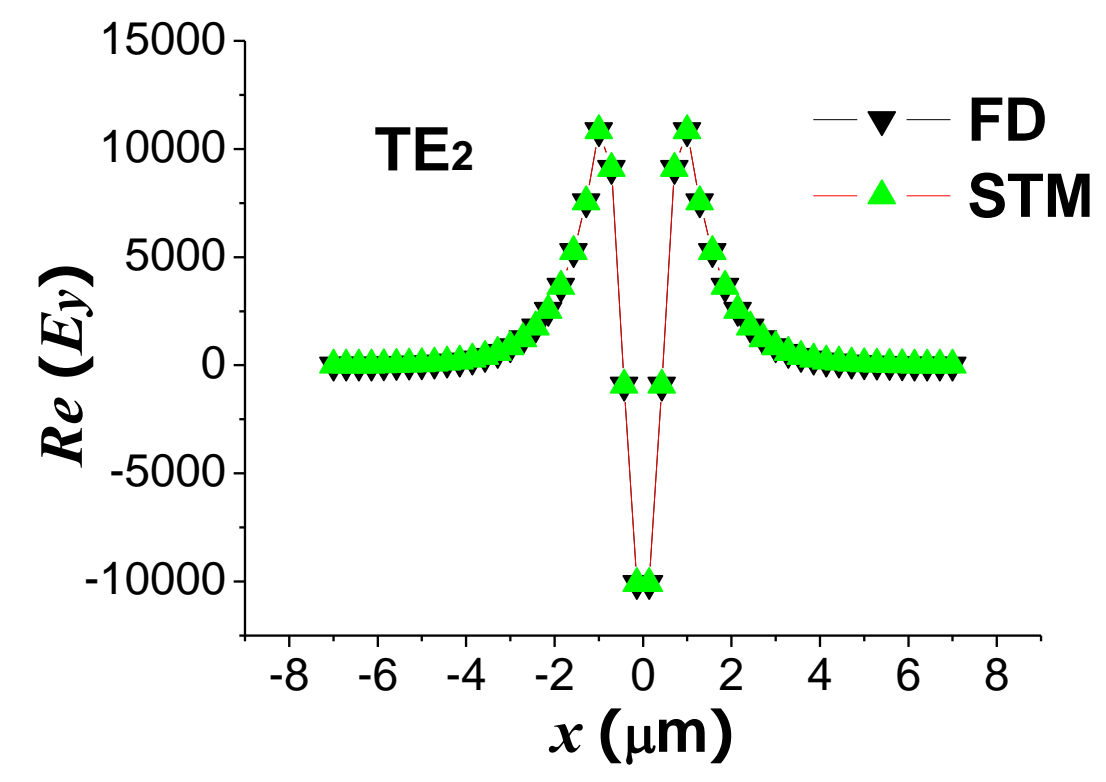

(c)

Figure 2.17. $E_{y}$ patterns of (a) $\mathrm{TE}_{0},(\mathrm{~b}) \mathrm{TE}_{1}$ and (c) TE2 in slab waveguide 2

Table 2 The comparisons of guided mode $N_{\text {eff }}$ calculated from conventional STM and HOFD in slab waveguide (Figure 2.4(b)) with $\mathrm{n} 1=3.3, \mathrm{n} 2=3.17,2 \mathrm{~S}=2 \mu \mathrm{m}$, and $\lambda$ $=1.55 \mu \mathrm{m}$ for $\mathrm{TM}$ polarization

\begin{tabular}{|r|r|r|r|}
\hline \multicolumn{1}{|l|}{ Mode } & TM0 & TM1 & TM2 \\
\hline FD & 3.2855 & 3.2438 & 3.1846 \\
\hline STM & 3.2855 & 3.2439 & 3.1846 \\
\hline
\end{tabular}




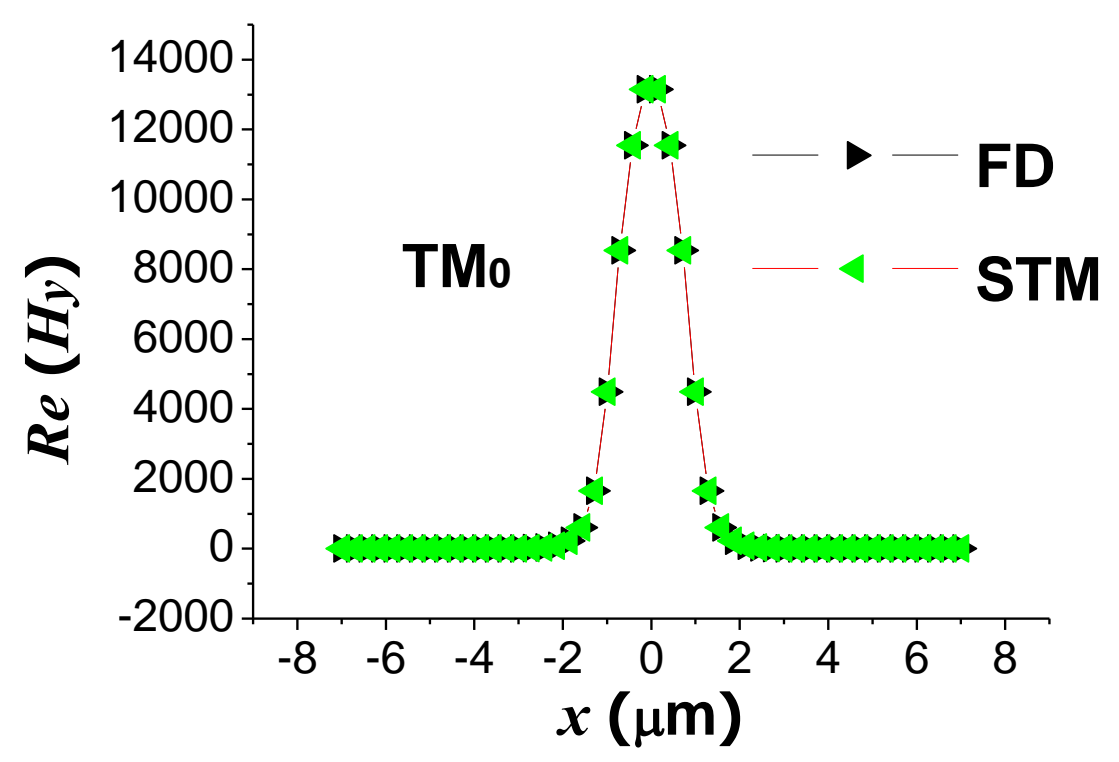

(a)

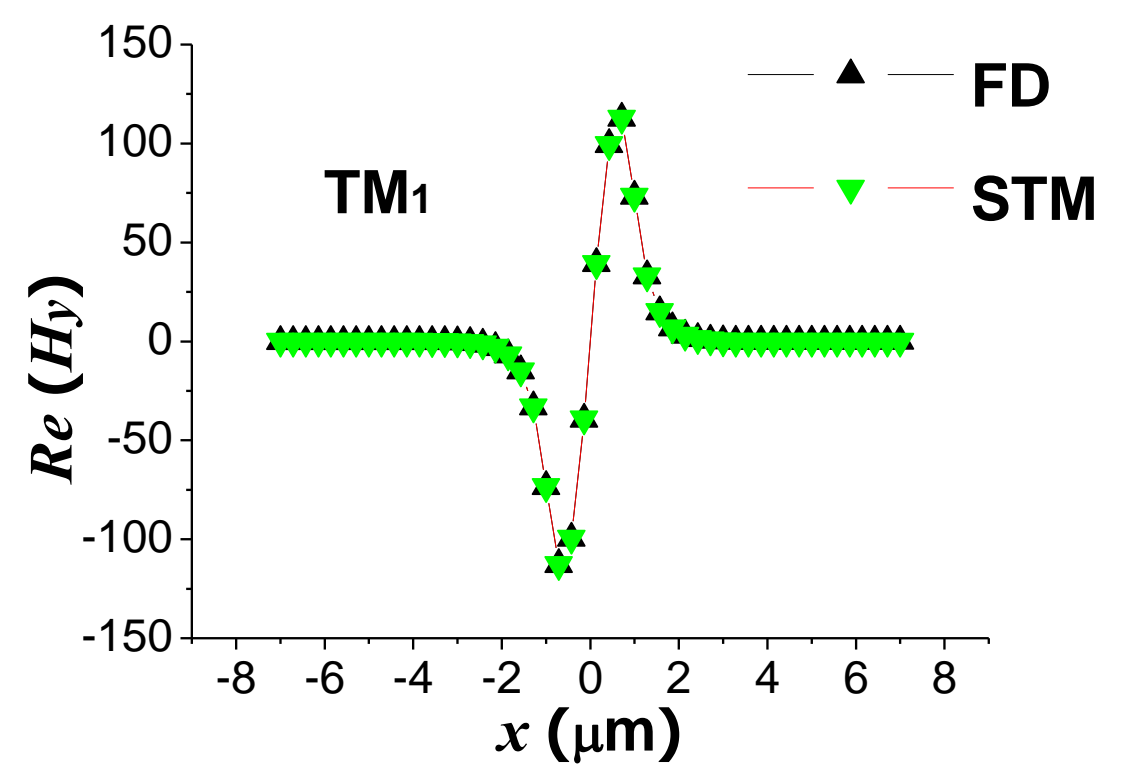

(b) 


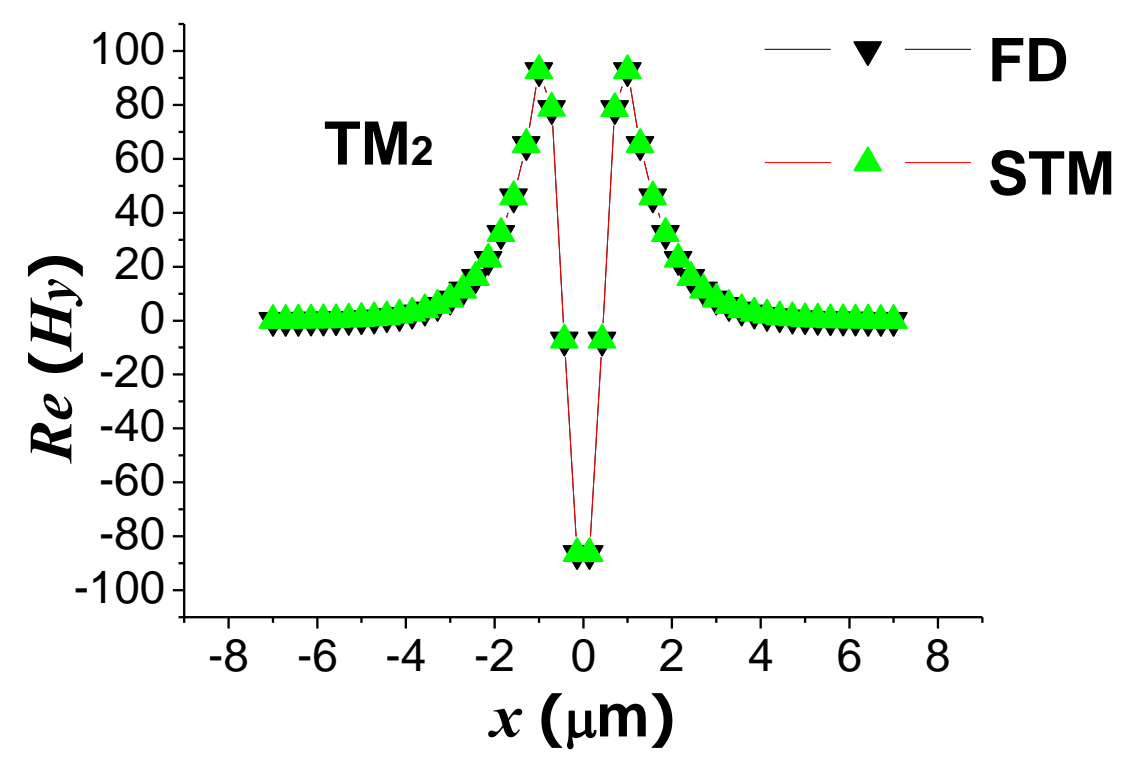

(c)

Figure 2.18. $H_{y}$ patterns of (a) $\mathrm{TM}_{0},(\mathrm{~b}) \mathrm{TM}_{1}$ and (c) $\mathrm{TM} 2$ in slab waveguide 2

\subsection{Validation of HOFD Scheme in Hollow Waveguide}

In order to further validate HOFD scheme, we consider the hallow waveguide in

Figure 2.10, with dair $=5 \mu \mathrm{m}, \mathrm{dPML}=2 \mu \mathrm{m}, \lambda=1.55 \mu \mathrm{m}, \quad R_{P M L}=1 \mathrm{e}-3$. 


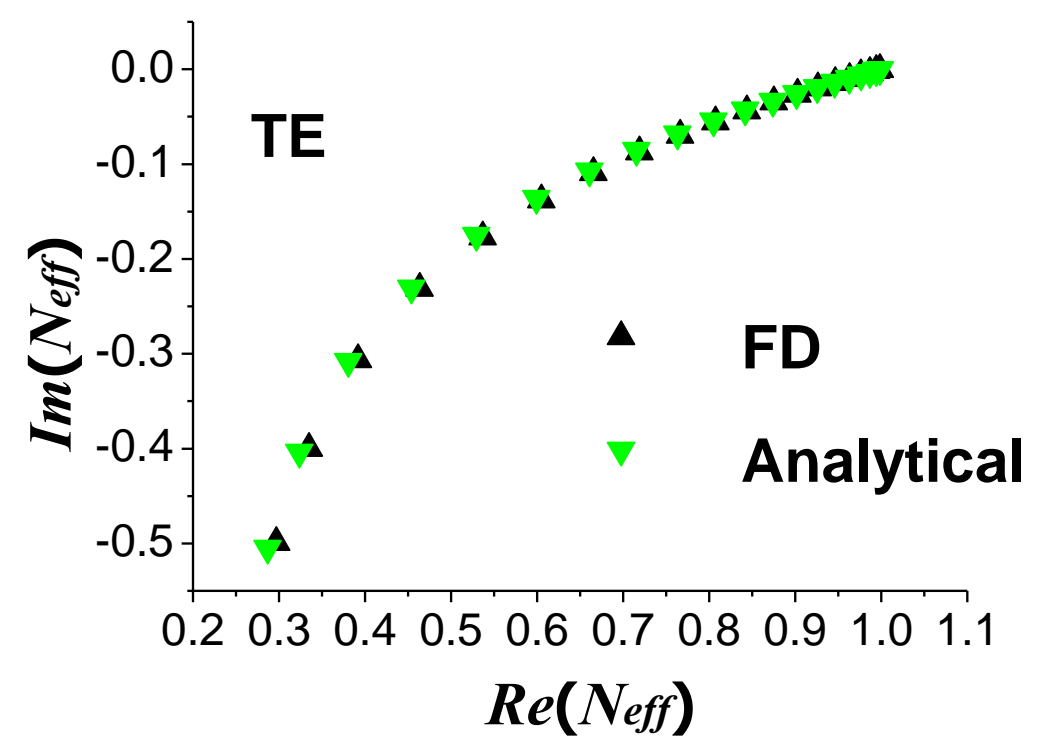

(a)

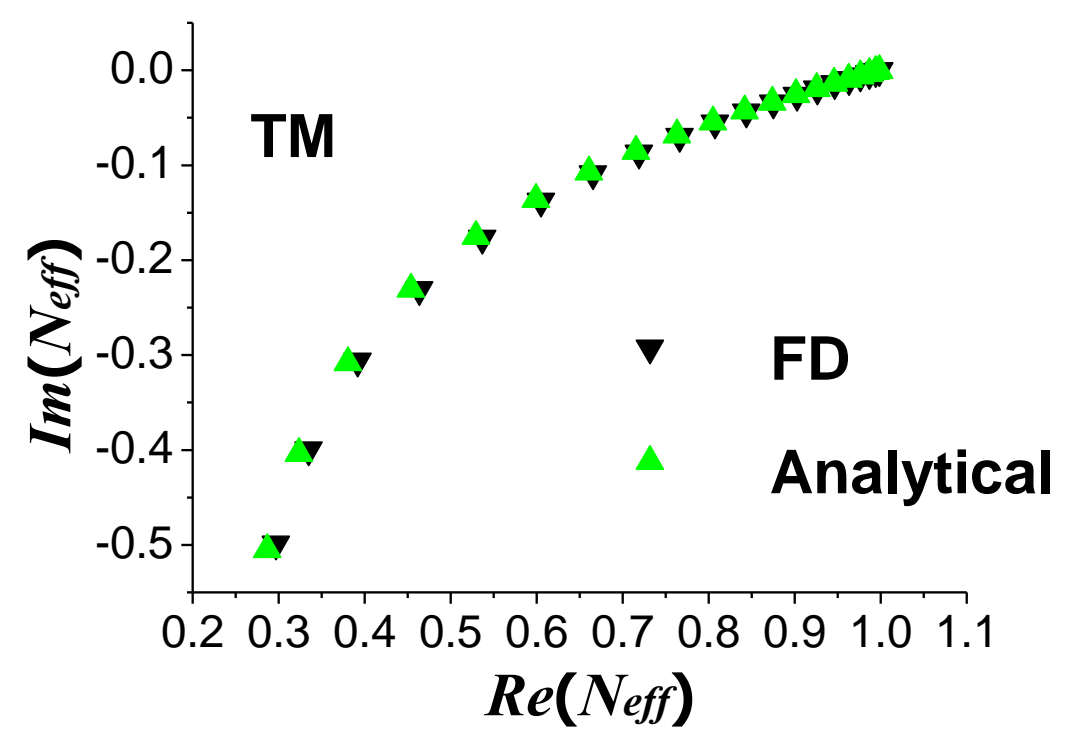

(b)

Figure 2.19. Mode spectrums of in (a)TE and (b)TM cases in hollow waveguide

Figure 2.20 and Figure 2.21 show the mode profiles of the hollow waveguide mentioned above in TE and TM polarization cases, respectively. And we compare the 
results from HOFD scheme with those obtained by the analytical solutions studied in

Section 2.33.

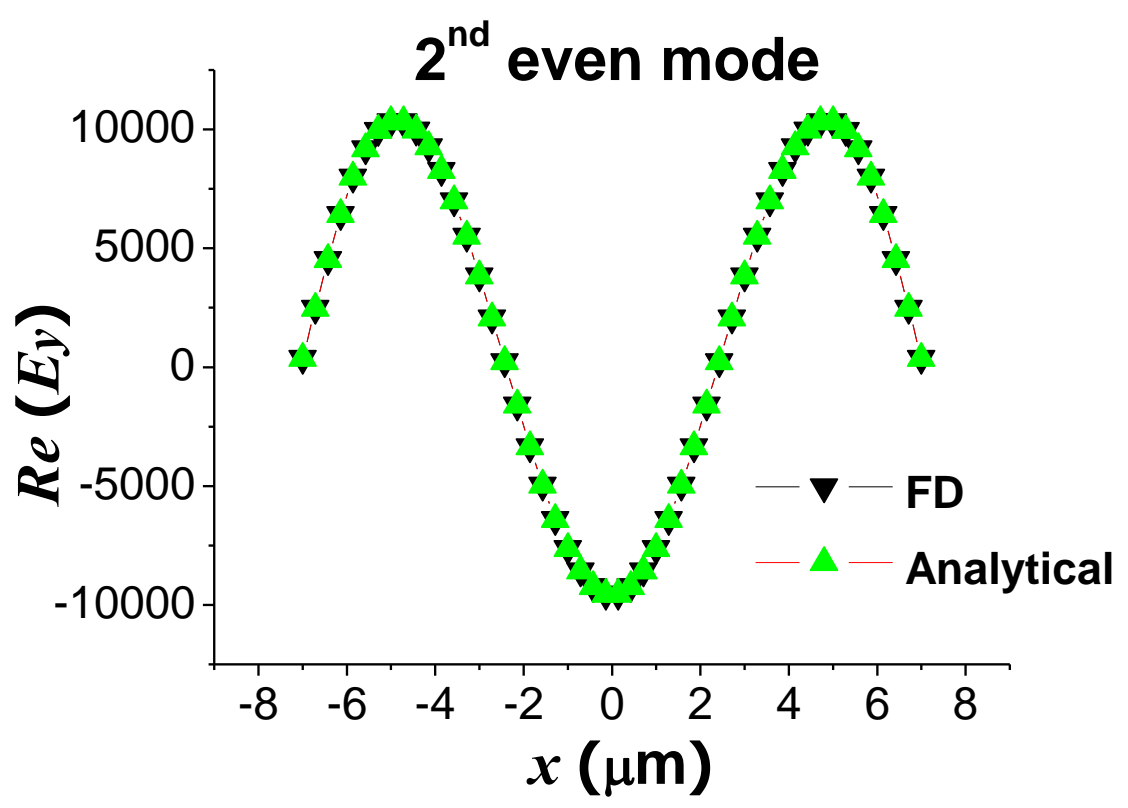

(a)

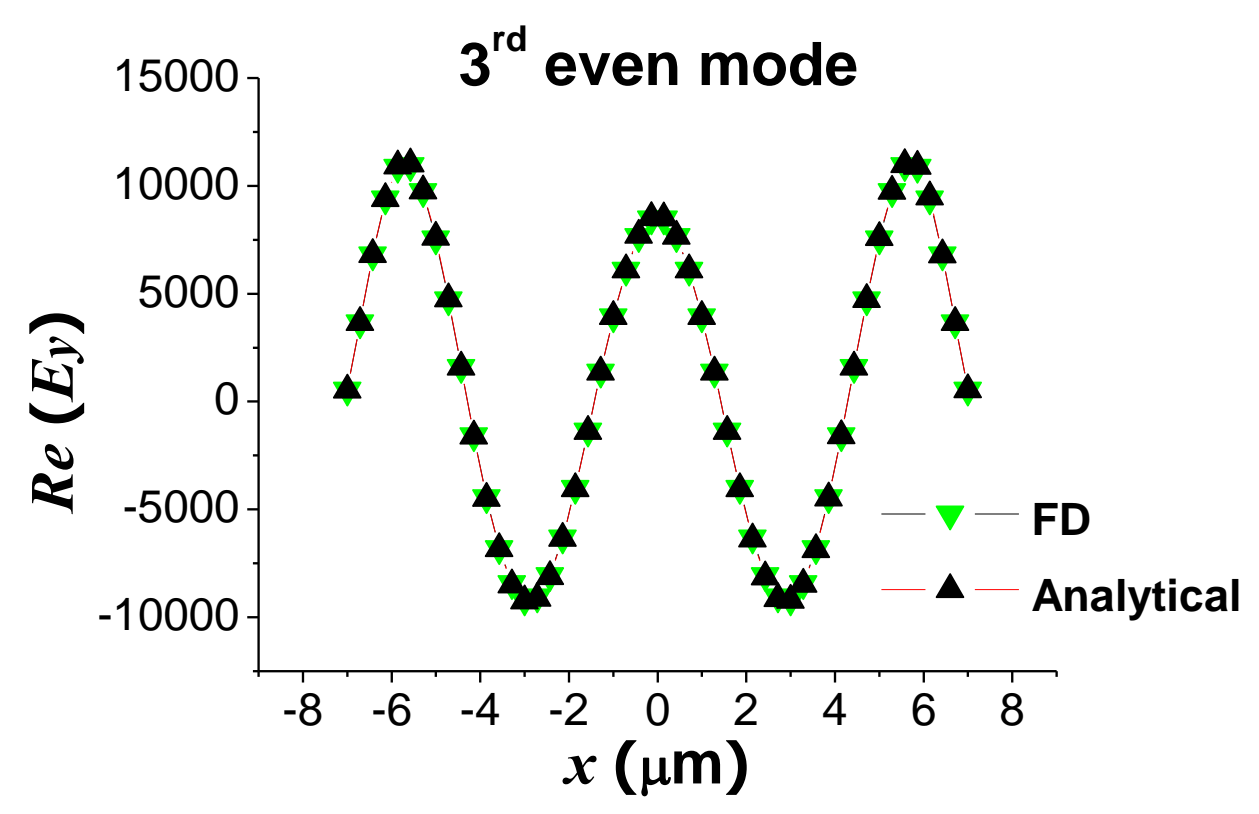

(b) 


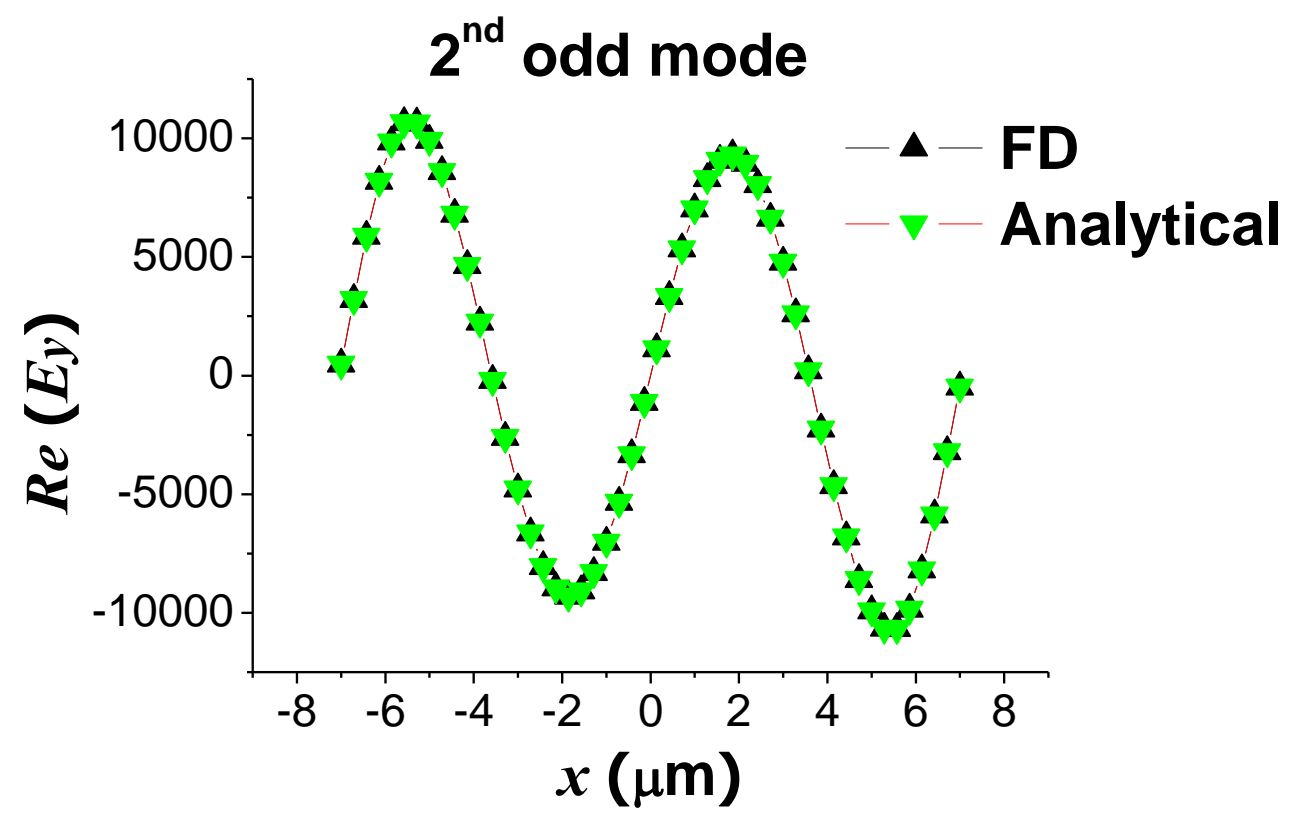

(c)

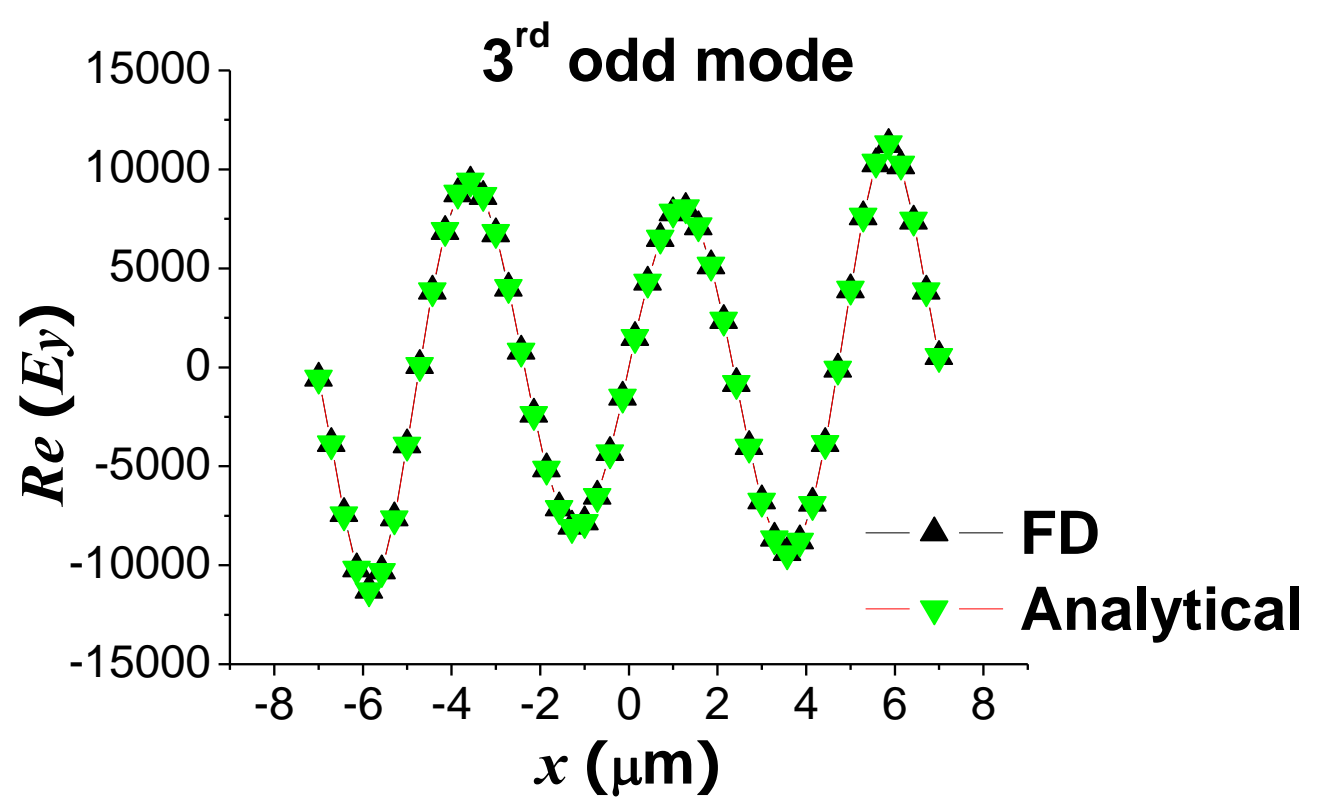

(d)

Figure 2.20. $E_{y}$ patterns of (a) the $2^{\text {nd }}$ even mode ,(b) the $3^{\text {rd }}$ even mode, (c) the $2^{\text {nd }}$ odd mode and (d) the $3^{\text {rd }}$ odd mode in hollow waveguide for TE polarization. 


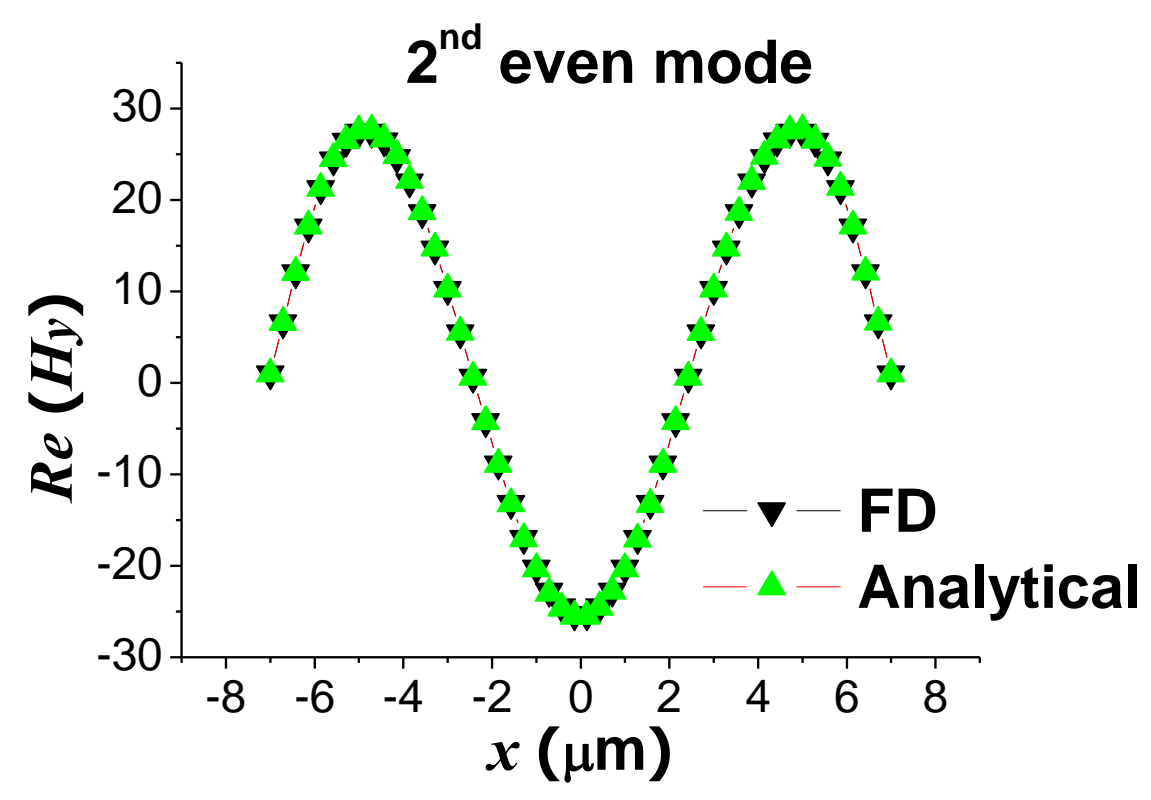

(a)

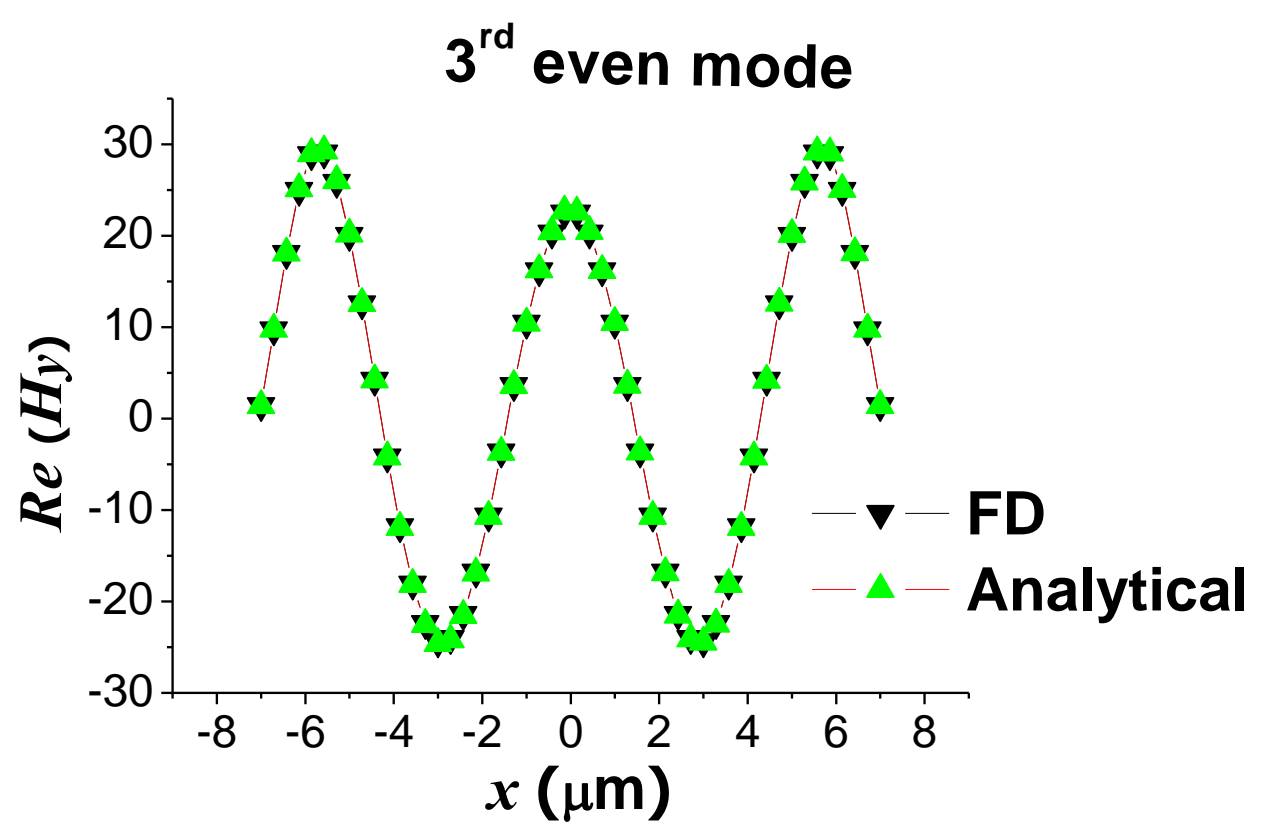

(b) 


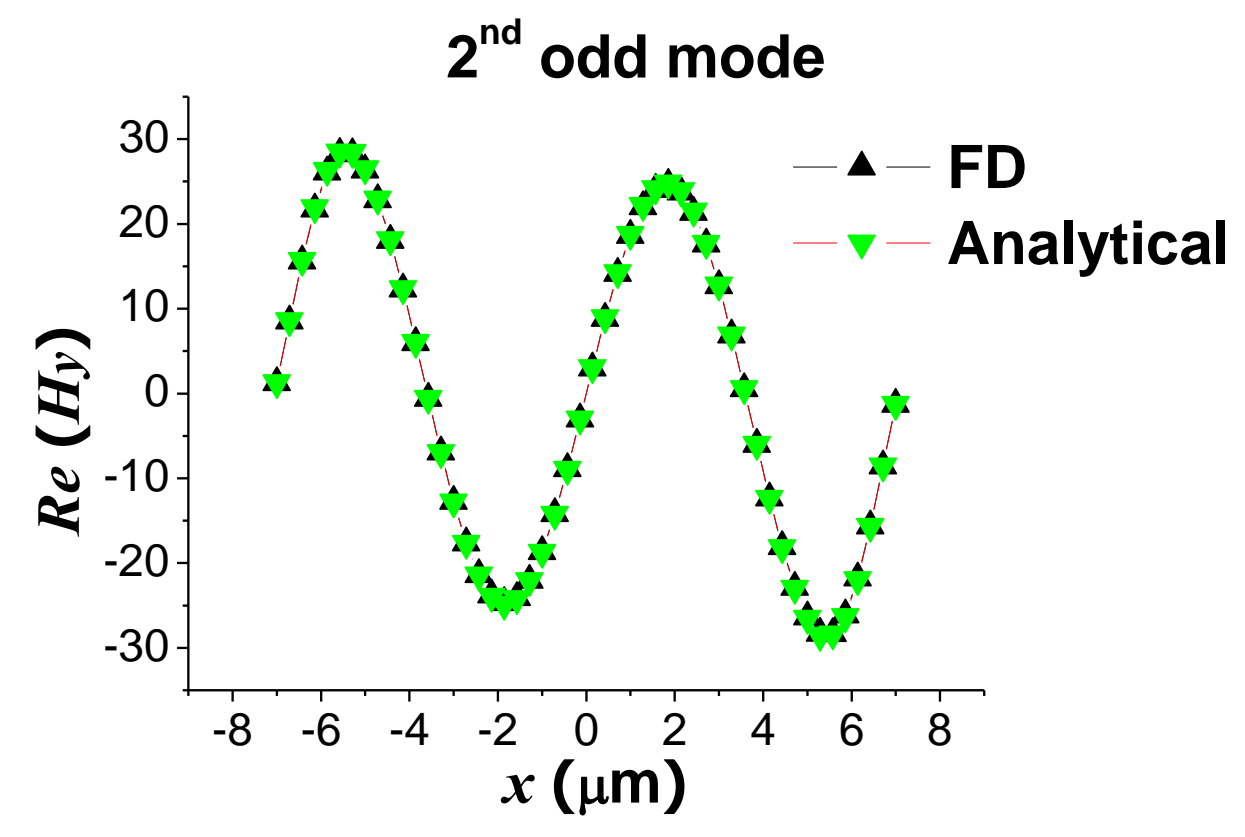

(c)

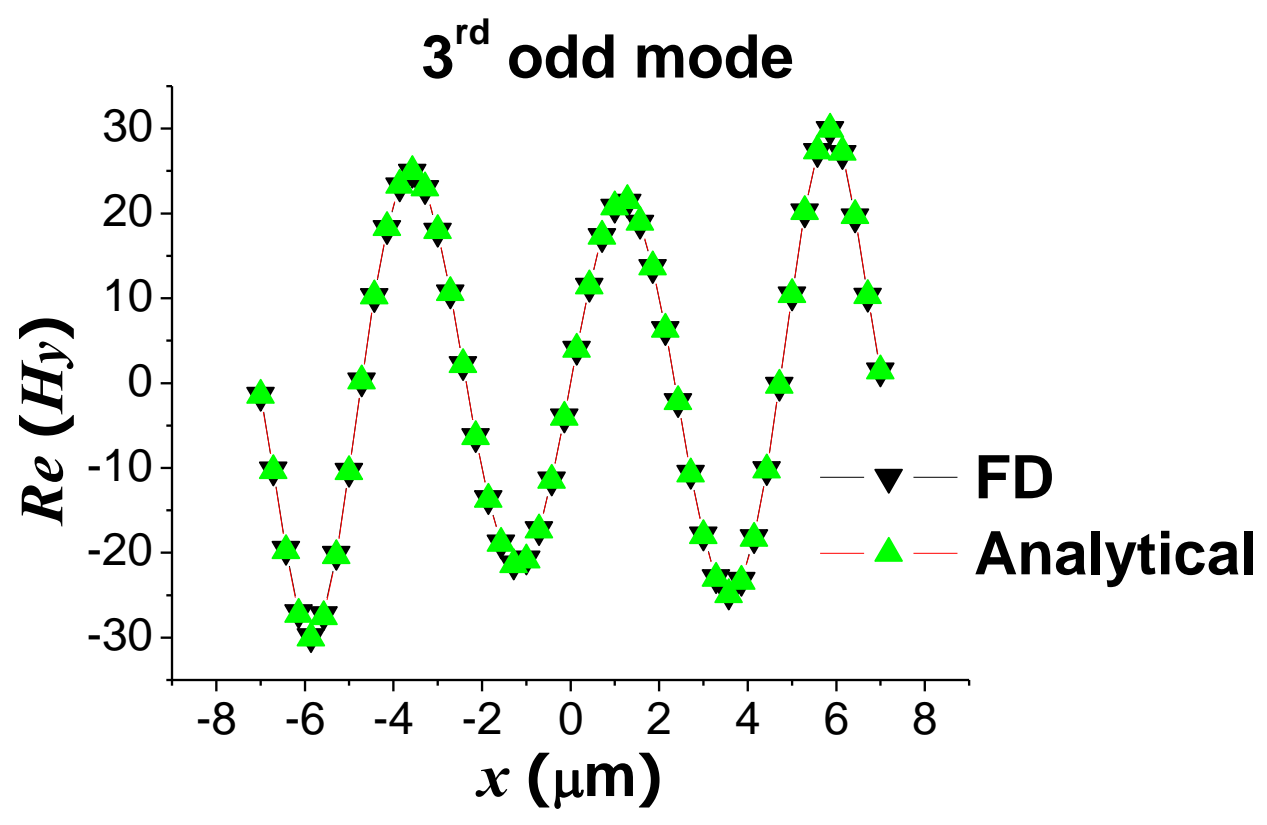

(d)

Figure 2.21. $H_{y}$ patterns of (a) the $2^{\text {nd }}$ even mode, (b) the $3^{\text {rd }}$ even mode , (c) the $2^{\text {nd }}$ odd mode and (d) the $3^{\text {rd }}$ odd mode in hollow waveguide for TM polarization. 


\section{Chapter 3 \\ Complex Mode Matching Method (CMMM)}

\subsection{Introduction}

Among the extensive methods of theoretical modeling and analysis of optical waveguide, Coupled Mode Theory (CMT)/Coupled-wave approach (CWA) are physically intuitive and mathematically convenient. However the accuracy of CMT/CWA can only be maintained in waveguide structures with small enough perturbation. Instead MMM is generally considered efficient and accurate method based on the tangential field continuity and the mode orthogonality conditions. The power flow and field pattern are obtained through the linking of different sections of uniform regions by the cascading of transfer matrices or scattering matrices.

\subsection{Transfer Matrix Formulation}

Figure 3.1 shows a single waveguide discontinuity between 2-D waveguide A and B which are terminated with PML and PRB on each side. $\hat{X}$ is the transverse direction, and $\hat{Z}$ is the longitudinal direction 

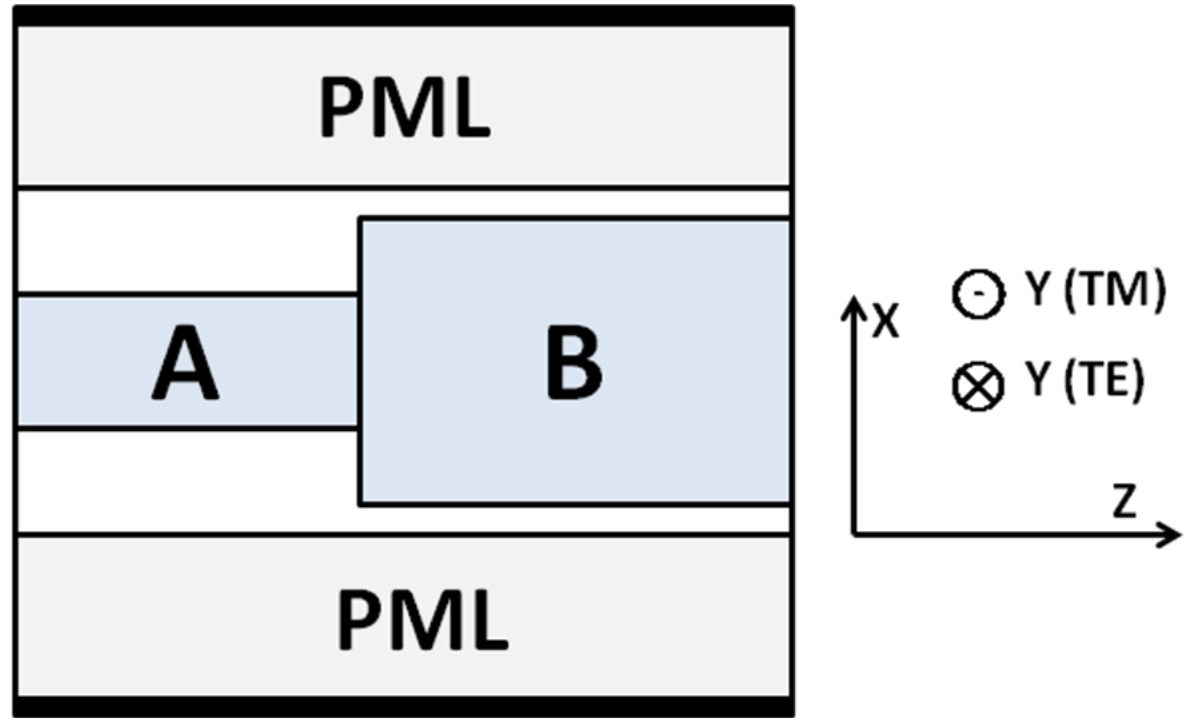

Figure 3.1 The schematic of a waveguide discontinuity between 2-D waveguides

We utilize HOFD scheme combined with complex coordinate stretching formulations of PML to get the mode spectrums. We assume $\mathrm{N}$ modes in waveguide $\mathrm{A}$ and $\mathrm{M}$ modes in waveguide $\mathrm{B}$ are used in the mode expansion. The transverse fields on both sides of the waveguide junction can be expressed approximately in term of eigenmodes in waveguide A and B [29-32]

$$
\begin{aligned}
& \vec{E}_{t}^{A}(x, z)=\sum_{n=1}^{N}\left(a_{n}^{+} e^{-j \beta_{n}^{A} Z}+a_{n}^{-} e^{j \beta_{n}^{A} Z}\right) \vec{e}_{t n}^{A}(x) \\
& \vec{H}_{t}^{A}(x, z)=\sum_{n=1}^{N}\left(a_{n}^{+} e^{-j \beta_{n}^{A} Z}+a_{n}^{-} e^{j \beta_{n}^{A} Z}\right) \vec{h}_{t n}^{A}(x) \\
& \vec{E}_{t}^{B}(x, z)=\sum_{n=1}^{N}\left(b_{n}^{+} e^{-j \beta_{n}^{B} Z}+b_{n}^{-} e^{j \beta_{n}^{B} Z}\right) \vec{e}_{t n}^{B}(x) \\
& \vec{H}_{t}^{B}(x, z)=\sum_{n=1}^{N}\left(b_{n}^{+} e^{-j \beta_{n}^{B} Z}+b_{n}^{-} e^{j \beta_{n}^{B} Z}\right) \vec{h}_{t n}^{B}(x)
\end{aligned}
$$

where $\mathrm{t}$ represents transverse components, $\vec{E}_{t}^{A}(x, z)\left(\vec{E}_{t}^{B}(x, z)\right)$ and $\vec{H}_{t}^{A}(x, z)$ $\left(\vec{H}_{t}^{B}(x, z)\right)$ are the transverse electric and magnetic field of waveguide A (B), respectively. $\quad \beta_{n(m)}^{A(B)}$ is the propagation constant of the $\mathrm{n}^{\text {th }}\left(\mathrm{m}^{\mathrm{th}}\right)$ mode in waveguide $\mathrm{A}$ (B). $\vec{e}_{t n(m)}^{A(B)}$ and $\vec{h}_{t n(m)}^{A(B)}$ denote transverse modal electric and magnetic field vectors of 
waveguide A (B), respectively. $a_{n}^{+}\left(b_{m}^{+}\right)$and $a_{n}^{-}\left(b_{m}^{-}\right)$are longitudinally forward and backward propagating wave amplitudes of $n^{\text {th }}\left(\mathrm{m}^{\text {th }}\right)$ mode of waveguide $A(B)$, respectively.

According to the continuity of tangential components of electric and magnetic field at the interface of two adjacent passive waveguides, we obtain [31-32]

$$
\begin{aligned}
& \sum_{n=1}^{N}\left(a_{n}^{+}+a_{n}^{-}\right) \vec{e}_{t n}^{A}(x)=\sum_{m=1}^{M}\left(b_{m}^{+}+b_{m}^{-}\right) \vec{e}_{t m}^{B}(x) \\
& \sum_{n=1}^{N}\left(a_{n}^{+}-a_{n}^{-}\right) \vec{h}_{t n}^{A}(x)=\sum_{m=1}^{M}\left(b_{m}^{+}-b_{m}^{-}\right) \vec{h}_{t m}^{B}(x)
\end{aligned}
$$

We define the cross product of field vectors $\vec{E}$ and $\vec{H}$ as [31-32]

$$
\langle E, H\rangle=\frac{1}{2} \int E \times H \cdot \vec{z} d x
$$

The integration is over the waveguide cross-section. We cross product both sides of (3.5) with $\vec{h}_{t g}^{B}$, and cross product both sides of (3.6) with $\vec{e}_{t g}^{B}[31-32]$

$$
\begin{aligned}
& \sum_{n=1}^{N}\left(a_{n}^{+}+a_{n}^{-}\right)\left\langle\vec{e}_{t n}^{A}, \vec{h}_{t g}^{B}\right\rangle=\sum_{m=1}^{M}\left(b_{m}^{+}+b_{m}^{-}\right)\left\langle\vec{e}_{t m}^{B}, \vec{h}_{t g}^{B}\right\rangle \\
& \sum_{n=1}^{N}\left(a_{n}^{+}-a_{n}^{-}\right)\left\langle\vec{e}_{t g}^{B}, \vec{h}_{t n}^{A}\right\rangle=\sum_{m=1}^{M}\left(b_{m}^{+}-b_{m}^{-}\right)\left\langle\vec{e}_{t g}^{B}, \vec{h}_{t m}^{B}\right\rangle
\end{aligned}
$$

According to the modal orthogonality for waveguides terminated with PML and PRB

$$
\begin{gathered}
\left\langle\vec{e}_{t n}^{A(B)}, \vec{h}_{t m}^{A(B)}\right\rangle=0, \quad m \neq n \\
\left\langle\vec{e}_{t n}^{A(B)}, \vec{h}_{t n}^{A(B)}\right\rangle=N_{n}
\end{gathered}
$$

$N_{n}$ is 1 for guided modes and a complex value for complex modes. Substituting (3.10), (3.11) into (3.8), (3.9), we get [31-32]

$$
\begin{aligned}
& b_{t g}^{+}=\sum_{n=1}^{N} a_{n}^{+}\left[\frac{\left\langle\vec{e}_{t n}^{A}, \vec{h}_{t g}^{B}\right\rangle+\left\langle\vec{e}_{t g}^{B}, \vec{h}_{t n}^{A}\right\rangle}{2\left\langle\vec{e}_{t g}^{B}, \vec{h}_{t g}^{B}\right\rangle}\right]+\sum_{n=1}^{N} a_{n}^{-}\left[\frac{\left\langle\vec{e}_{t n}^{A}, \vec{h}_{t g}^{B}\right\rangle-\left\langle\vec{e}_{t g}^{B}, \vec{h}_{t n}^{A}\right\rangle}{2\left\langle\vec{e}_{t g}^{B}, \vec{h}_{t g}^{B}\right\rangle}\right] \\
& b_{t g}^{-}=\sum_{n=1}^{N} a_{n}^{+}\left[\frac{\left\langle\vec{e}_{t n}^{A}, \vec{h}_{t g}^{B}\right\rangle-\left\langle\vec{e}_{t g}^{B}, \vec{h}_{t n}^{A}\right\rangle}{2\left\langle\vec{e}_{t g}^{B}, \vec{h}_{t g}^{B}\right\rangle}\right]+\sum_{n=1}^{N} a_{n}^{-}\left[\frac{\left\langle\vec{e}_{t n}^{A}, \vec{h}_{t g}^{B}\right\rangle+\left\langle\vec{e}_{t g}^{B}, \vec{h}_{t n}^{A}\right\rangle}{2\left\langle\vec{e}_{t g}^{B}, \vec{h}_{t g}^{B}\right\rangle}\right]
\end{aligned}
$$

Consequently we can further write them in more compact form 


$$
\left|\begin{array}{l}
B^{+} \\
B^{-}
\end{array}\right|=T_{B A}\left|\begin{array}{l}
A^{+} \\
A^{-}
\end{array}\right|=\left|\begin{array}{ll}
T_{11} & T_{12} \\
T_{21} & T_{22}
\end{array}\right|\left|\begin{array}{l}
A^{+} \\
A^{-}
\end{array}\right|
$$

where $B^{+}=\left|b_{1}^{+}, b_{2}^{+}, \ldots \ldots, b_{N}^{+}\right|^{T}, \quad B^{-}=\left|b_{1}^{-}, b_{2}^{-}, \ldots \ldots, b_{N}^{-}\right|^{T}$. Note that $T_{A B}=T_{B A}{ }^{\prime}$

In order to numerically validate the transfer matrix, we consider a 2-D slab waveguide junction in Figure 3.2. The fundamental mode is launched from the left port, and we validate the consistency of tangential components of electric (magnetic) field at the waveguide junction in TE (TM) polarization case in Figure 3.3 (Figure 3.4). The wavelength is $1.55 \mu \mathrm{m}$, the mesh size is $20 \mathrm{~nm}$, and $R_{P M L}$ is $1 \mathrm{e}-3.60$ modes are considered.

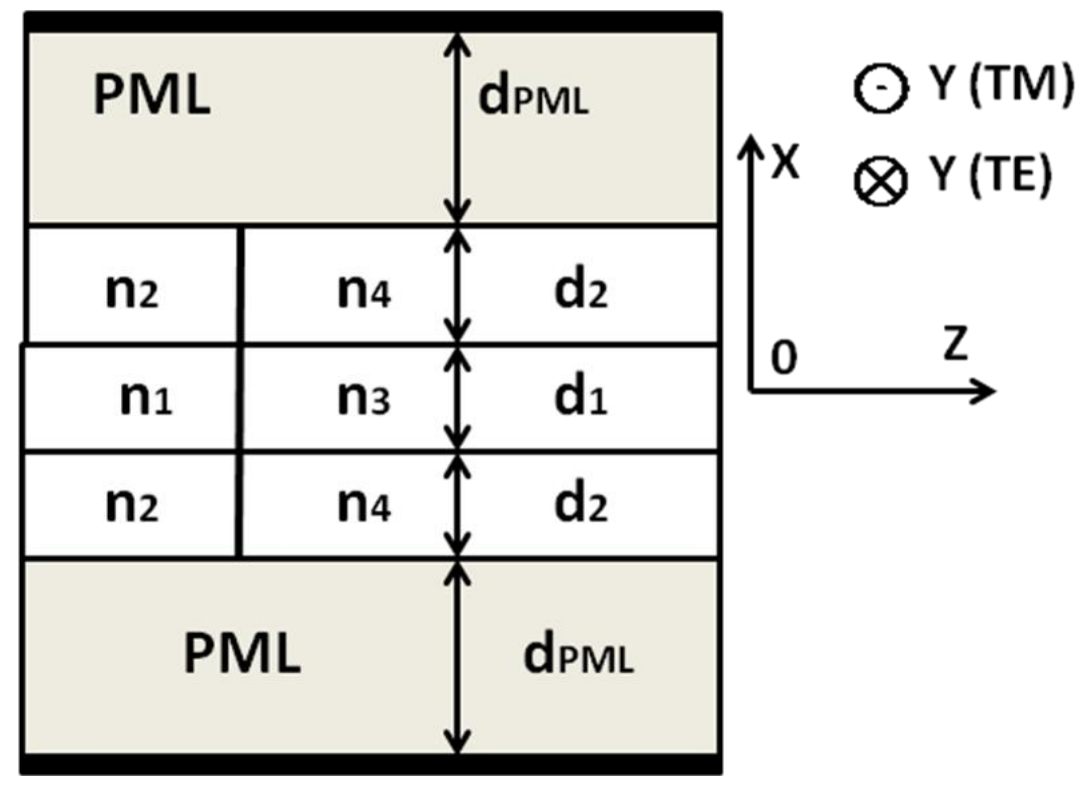

Figure 3.2. The schematic of two adjacent 2-D slab waveguide terminated with PML and PRB. $\mathrm{n} 1=3.3, \mathrm{n} 2=3.17, \mathrm{n} 3=3.4, \mathrm{n} 4=3.2, \mathrm{~d} 1=0.5 \mu \mathrm{m}, \mathrm{d} 2=2 \mu \mathrm{m}, \mathrm{dPML}=5 \mu \mathrm{m}$. 


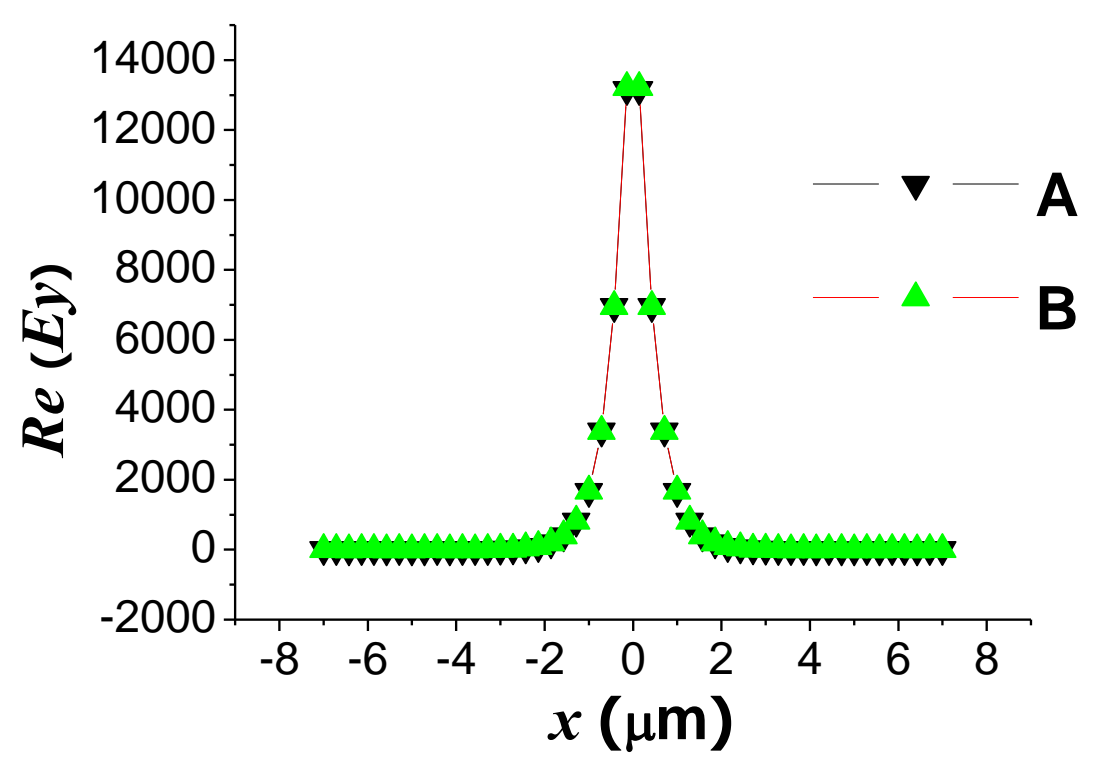

Figure 3.3. The continuity test of $E_{y}$ patterns at the waveguide junction (Figure 3.2) for TE polarization

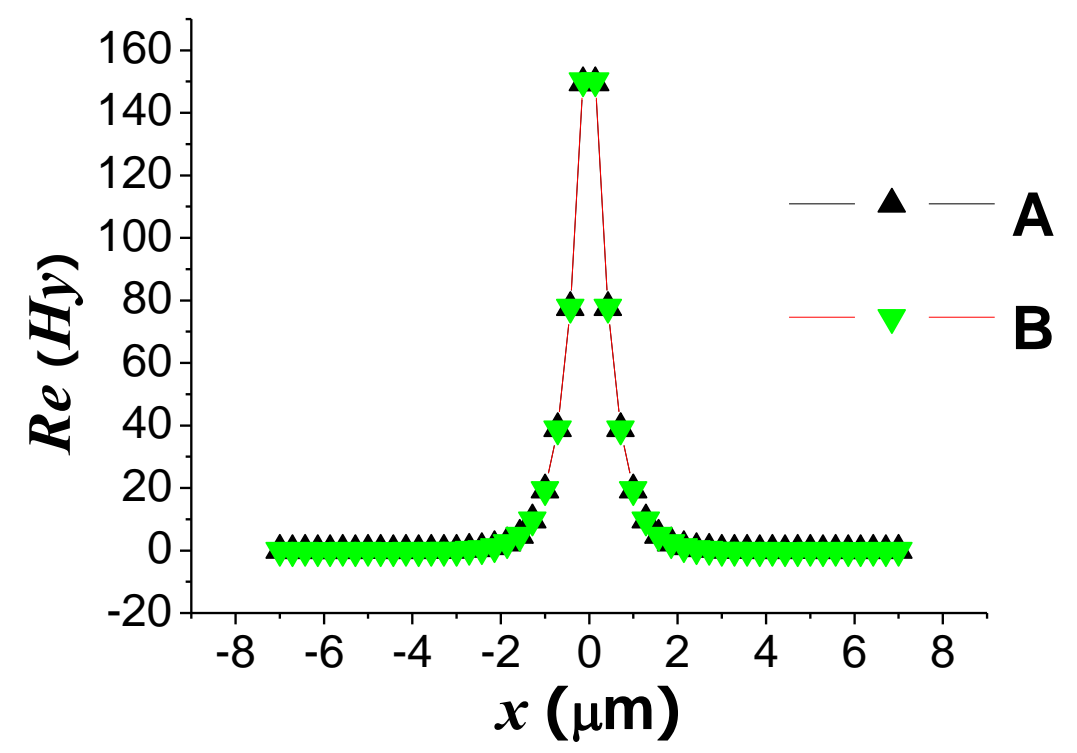

Figure 3.4. The continuity test of $H_{y}$ patterns at the waveguide junction (Figure 3.2) for TM polarization

\subsection{Scattering Matrix Formulation}

Considering the waveguide junction in Figure 3.1 


$$
\begin{aligned}
& B^{+}=T_{11} A^{+}+T_{12} A^{-} \\
& B^{-}=T_{21} A^{+}+T_{22} A^{-}
\end{aligned}
$$

We multiply $T_{12}^{\prime}$ on the two sides of (3.15)

$$
T_{12}^{\prime} B^{+}-A^{-}=T_{12}^{\prime} T_{11} A^{+}
$$

Then we multiply $T_{22}^{\prime}$ on both sides of (3.16)

$$
A^{-}=T_{11}^{\prime} B^{-}-T_{11}^{\prime} T_{12} A^{+}
$$

By combining (3.17) and (3.18), we have

$$
\begin{gathered}
B^{+}=\left(T_{11}-T_{12} T_{22}^{\prime} T_{21}\right) A^{+}+T_{12} T_{22}^{\prime} B^{-} \\
A^{-}=-T_{22}^{\prime} T_{21} A^{+}+T_{22}^{\prime} B^{-}
\end{gathered}
$$

We further write it into matrix form

$$
\left|\begin{array}{l}
B^{+} \\
A^{-}
\end{array}\right|=S_{A B}\left|\begin{array}{l}
A^{+} \\
B^{-}
\end{array}\right|=\left|\begin{array}{ll}
S_{11} & S_{12} \\
S_{21} & S_{22}
\end{array}\right|\left|\begin{array}{l}
A^{+} \\
B^{-}
\end{array}\right|
$$

which is the well-known scattering matrix. In a homogeneous region called C (Figure $3.5)$

$$
\left|\begin{array}{c}
B^{+} \\
A^{-}
\end{array}\right|=S_{c}\left|\begin{array}{l}
A^{+} \\
B^{-}
\end{array}\right|=\left|\begin{array}{cc}
S_{p} & 0 \\
0 & S_{p}
\end{array}\right|\left|\begin{array}{l}
A^{+} \\
B^{-}
\end{array}\right|
$$

where $S_{p}=\operatorname{diag}\left(\left|e^{-j \beta_{1} L}, e^{-j \beta_{2} L}, \ldots \ldots, e^{-j \beta_{N} L}\right|\right), \quad \mathrm{L}$ is the longitudinal length of $\mathrm{C}$

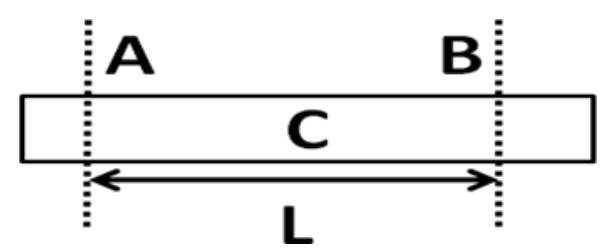

Figure 3.5. The schematic of a homogeneous 2-D region $\mathrm{C}$ 


\subsection{Periodic Doubling Scheme}

The Bragg grating has wide applications in chip-fiber coupling, reflector, and so on [51]. As the Bragg grating structure contains a large number of symmetric cells, the scattering matrix holds more stability than the transfer matrix.

The cascading of scattering matrix can be denoted by the operator

' $\otimes$ '.Assuming there are three sequent discontinuities noted as $\mathrm{n}, \mathrm{m}$ and $\mathrm{k}$, we have

$$
\begin{gathered}
\left|\begin{array}{c}
A_{m}^{+} \\
A_{n}^{-}
\end{array}\right|=S_{n m}\left|\begin{array}{c}
A_{n}^{+} \\
A_{m}^{-}
\end{array}\right|=\left|\begin{array}{ll}
T_{n m} & R_{m n} \\
R_{n m} & T_{m n}
\end{array}\right|\left|\begin{array}{c}
A_{n}^{+} \\
A_{m}^{-}
\end{array}\right| \\
\left|\begin{array}{c}
A_{k}^{+} \\
A_{m}^{-}
\end{array}\right|=S_{m k}\left|\begin{array}{c}
A_{m}^{+} \\
A_{k}^{-}
\end{array}\right|=\left|\begin{array}{ll}
T_{m k} & R_{k m} \\
R_{m k} & T_{k m}
\end{array}\right|\left|\begin{array}{c}
A_{m}^{+} \\
A_{k}^{-}
\end{array}\right|
\end{gathered}
$$

We derive (3.23) to be

$$
\begin{gathered}
A_{m}^{+}=R_{m n} T_{m n}^{\prime} A_{n}^{-}+\left(T_{n m}-R_{m n} T_{m n}^{\prime} R_{n m}\right) A_{n}^{+} \\
A_{m}^{-}=T_{m n}^{\prime} A_{n}^{-}-T_{m n}^{\prime} R_{n m} A_{n}^{+}
\end{gathered}
$$

Substituting (3.25) and (3.26) into (3.24), we get the cascaded scattering matrix [31-32]

$$
\begin{gathered}
\left|\begin{array}{c}
A_{k}^{+} \\
A_{n}^{-}
\end{array}\right|=S_{n k}\left|\begin{array}{c}
A_{n}^{+} \\
A_{k}^{-}
\end{array}\right|=\left|\begin{array}{cc}
T_{n k} & R_{k n} \\
R_{n k} & T_{k n}
\end{array}\right| \begin{array}{c}
A_{n}^{+} \\
A_{k}^{-}
\end{array} \mid \\
T_{n k}=T_{m k}\left(I-R_{m n} R_{m k}\right)^{\prime} T_{n m}^{\prime} \\
R_{k n}=T_{m k}\left(I-R_{m n} R_{m k}\right)^{\prime} R_{m n} T_{k m}+R_{k m} \\
R_{n k}=T_{m n}\left(I-R_{m k} R_{m n}\right)^{\prime} R_{m k} T_{n m}+R_{n m} \\
T_{k n}=T_{m n}\left(I-R_{m k} R_{m n}\right)^{\prime} T_{k m}
\end{gathered}
$$

For the 2-D Bragg grating shown in Figure 3.6, the scattering matrix for the a unit cell is 


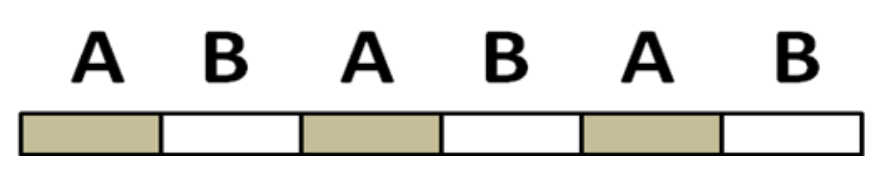

Figure 3.6. The schematic of a 2-D Bragg grating

$$
S_{\text {cell }}=S_{A} \otimes S_{A B} \oplus S_{B} \otimes S_{B A}
$$

If the Bragg grating contains $\mathrm{N}$ periods, the scattering for the whole periodic structure is [31-32]

$$
S_{\text {total }}=\underbrace{S_{\text {cell }} \otimes S_{\text {cell }} \otimes \ldots \ldots . \oplus S_{\text {cell }}}_{N}
$$

Utilizing the periodic doubling algorithm, the number of scattering matrix cascading operations is $\left(4^{N}-N-3\right)$ less than that from layer-by-layer cascading. 


\section{Chapter 4 \\ Complex Smooth Transition Method (STM)}

\subsection{Introduction}

Multi-layer planar waveguide plays a crucial rule in photonic devices and integrated circuits. Determination and optimization of guided and radiation modes in multi-layer planar waveguide are necessary, because both guided and radiation modes both are essential for expanding arbitrary field of open waveguide in many cases. In STM, the implicit characteristic function $f\left(\beta^{2}\right)=0$ is obtained from cascading of transfer matrices. The solving of this transcendental equation is usually through the Newton's root searching in complex plane.

The conventional smooth transition method is to assume artificial boundaries outside the 2-D multi-layer stacks. When the artificial boundaries are closed, the obtained box modes (guided or evanescent) serve as the initial guess of the following Newton's search which will be ended when the boundaries are open. However, the leaky modes in open waveguide represents are unbounded by nature and hence lack the usual characteristics of normal guided modes in terms of orthogonality and normalization [40].

On the contrast, complex STM utilizes the multi-layer waveguide structures always enclosed by PML and PRB. The transfer matrix establishing the governing equation is modified in the sense that effective thickness of PML is complex. The box 
modes when there is no absorption in PML medium are the initial guesses for Newton's searching. As $R_{P M L}$ gradually turns to be small enough, the complex and guided modes are finally defined.

In this chapter for the first time the semi-analytical solutions of complex mode spectrums and modal patterns are derived and compared with those from HOFD scheme.

\subsection{Formulas of Complex STM}

We consider a 2-D multi-layer planar waveguide terminated with PML and PRB on both sides (Figure 4.1). The number of layers is N, $\hat{X}$ and $\hat{Z}$ are the transverse and longitudinal directions, respectively. $n_{P M L, L}$ is the index of the PML on the left side, and $n_{P M L, R}$ is that on the right side. $A_{L}^{+}$and $A_{L}^{-}$are the amplitudes of forward and backward propagating waves at the PRB on the left side, respectively. $A_{R}^{+}$and $A_{R}^{-}$are those on the right side. For an arbitrary $\mathrm{m}^{\text {th }}$ layer, $d_{m}$ and $n_{m}$ are the thickness and index of this layer, respectively. $A_{m}^{+}$and $A_{m}^{-}$represent the amplitudes of forward and backward propagating waves at the left boundary of the $\mathrm{m}^{\text {th }}$ layer, respectively. $A_{m+1}^{+}$and $A_{m+1}^{-}$are those at the right boundary of the $\mathrm{m}^{\text {th }}$ layer. The $\mathrm{X}$ axis's coordinate at the left boundary of the $\mathrm{m}^{\text {th }}$ layer is $x_{m}$, and $x_{m+1}$ is that of the right boundary of the $\mathrm{m}^{\text {th }}$ layer. 

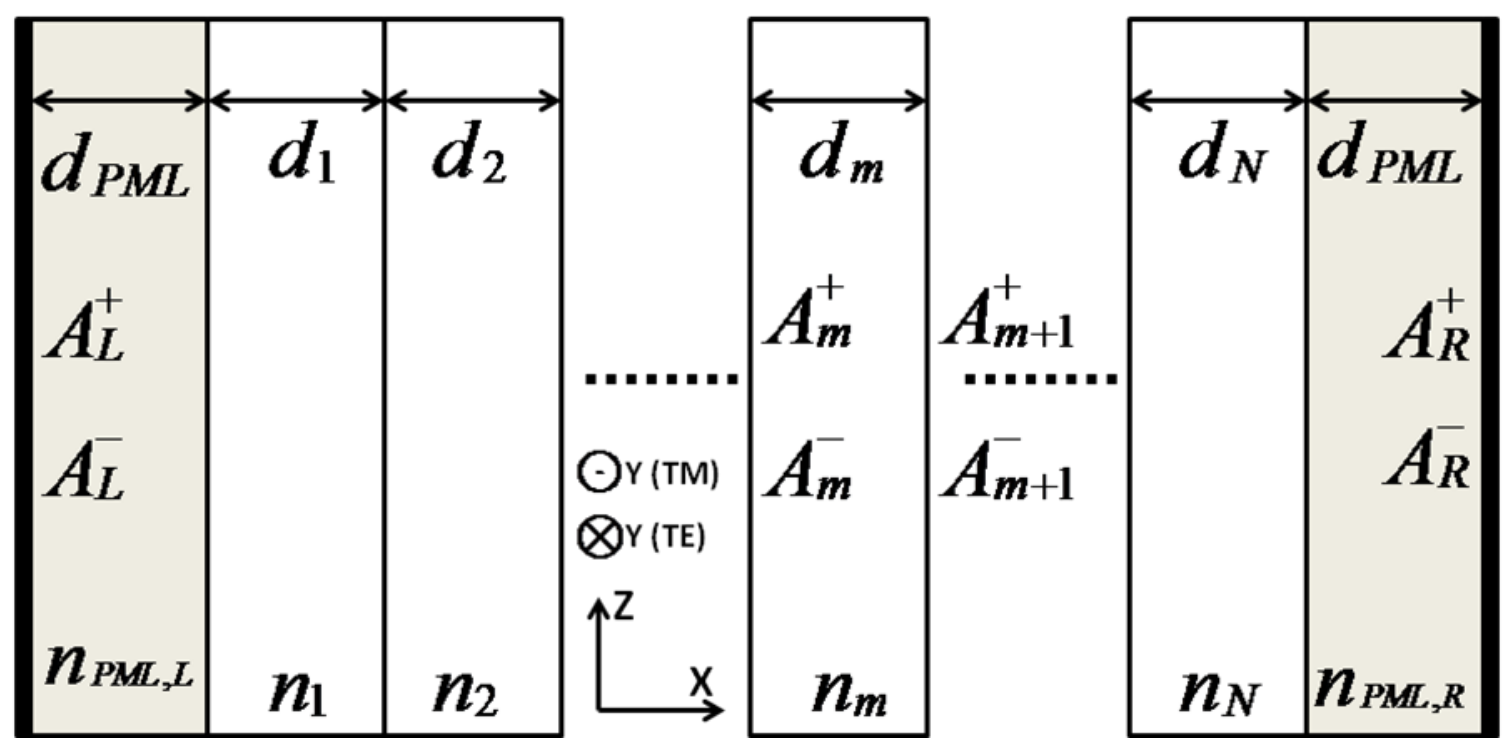

Figure 4.1. The schematic of a multi-layer planar waveguide terminated with PML and PRB on both sides

In the $\mathrm{m}^{\text {th }}$ layer

$$
\begin{gathered}
A_{m+1}^{+}=A_{m}^{+} e^{-j \kappa_{x, m} d_{m}} \\
A_{m}^{-}=A_{m+1}^{-} e^{-j_{\kappa_{x, m} d_{m}}} \\
\kappa_{x, m}=\sqrt{n_{m}^{2} k_{0}^{2}-\beta^{2}}
\end{gathered}
$$

For TE polarization, we define two variables $U=A^{+}+A^{-}, V=\kappa_{x}\left(A^{+}-A^{-}\right)$

In the $\mathrm{m}^{\text {th }}$ layer, we assume

$$
\left|\begin{array}{l}
U_{m} \\
V_{m}
\end{array}\right|=T_{m}\left|\begin{array}{l}
U_{m+1} \\
V_{m+1}
\end{array}\right|=\left|\begin{array}{ll}
T_{11} & T_{12} \\
T_{21} & T_{22}
\end{array}\right|\left|\begin{array}{l}
U_{m+1} \\
V_{m+1}
\end{array}\right|
$$

We derive (4.4) to be

$$
\begin{aligned}
& A_{m}^{+}+A_{m}^{-}=T_{11}\left(A_{m}^{+} e^{-j \kappa_{x, m} d_{m}}+A_{m}^{-} e^{j \kappa_{x, m} d_{m}}\right)+T_{12} \kappa_{x, m}\left(A_{m}^{+} e^{-j \kappa_{x, m} d_{m}}-A_{m}^{-} e^{j \kappa_{x,}}\right. \\
& A_{m}^{+}-A_{m}^{-}=\frac{T_{21}}{\kappa_{x, m}}\left(A_{m}^{+} e^{-j_{\kappa_{x, m} d_{m}}}+A_{m}^{-} e^{j_{\kappa_{x, m} d_{m}}}\right)+T_{22}\left(A_{m}^{+} e^{-j_{\kappa_{x, m} d_{m}}}-A_{m}^{-} e^{j_{\kappa_{x, m} d_{n}}}\right.
\end{aligned}
$$

From (4.5), we obtain

$$
1=T_{11} e^{-j_{\kappa_{x, m}} d_{m}}+T_{12} \kappa_{x, m} e^{-j_{\kappa_{x, m} d_{m}}}
$$




$$
1=T_{11} e^{j_{\kappa_{x, m}} d_{m}}-T_{12} \kappa_{x, m} e^{j_{\kappa_{x, m}} d_{m}}
$$

By substituting (4.7) into (4.8), we get

$$
\begin{gathered}
T_{11}=\cos \left(\kappa_{x, m} d_{m}\right) \\
T_{12}=\frac{j \sin \left(\kappa_{x, m} d_{m}\right)}{\kappa_{x, m}}
\end{gathered}
$$

From (4.6), we obtain

$$
\begin{aligned}
& 1=\frac{T_{21}}{\kappa_{x, m}} e^{-j_{\kappa_{x, m}} d_{m}}+T_{22} e^{-j_{\kappa_{x, m}} d_{m}} \\
& -1=\frac{T_{21}}{\kappa_{x, m}} e^{j_{\kappa_{x, m} d_{m}}}-T_{22} e^{j_{\kappa_{x, m} d_{m}}}
\end{aligned}
$$

Through substituting (4.11) into (4.12), we get

$$
\begin{gathered}
T_{22}=\cos \left(\kappa_{x, m} d_{m}\right) \\
T_{21}=j \kappa_{x, m} \sin \left(\kappa_{x, m} d_{m}\right)
\end{gathered}
$$

Finally, we summarize (4.4) to be

$$
T_{m}=\left|\begin{array}{cc}
\cos \left(\kappa_{x, m} d_{m}\right) & \frac{j \sin \left(\kappa_{x, m} d_{m}\right)}{\kappa_{x, m}} \\
j \kappa_{x, m} \sin \left(\kappa_{x, m} d_{m}\right) & \cos \left(\kappa_{x, m} d_{m}\right)
\end{array}\right|
$$

In PML region

$$
\begin{gathered}
\left|\begin{array}{l}
U_{L} \\
V_{L}
\end{array}\right|=T_{P M L}^{L}\left|\begin{array}{l}
U_{1} \\
V_{1}
\end{array}\right|=\left|\begin{array}{cc}
\cos \left(\kappa_{x, P M L}^{L} \tilde{d}_{P M L}^{L}\right) & \frac{j \sin \left(\kappa_{x, P M L}^{L} \tilde{d}_{P M L}^{L}\right)}{\kappa_{x, P M L}^{L}} \\
j \kappa_{x, P M L}^{L} \sin \left(\kappa_{x, P M L}^{L} \tilde{d}_{P M L}^{L}\right) & \cos \left(\kappa_{x, P M L}^{L} \tilde{d}_{P M L}^{L}\right)
\end{array}\right|\left|\begin{array}{l}
U_{1} \\
V_{1}
\end{array}\right| \\
\left|\begin{array}{l}
U_{N+1} \\
V_{N+1}
\end{array}\right|=T_{P M L}^{R}\left|\begin{array}{l}
U_{R} \\
V_{R}
\end{array}\right|=\left|\begin{array}{cc}
\cos \left(\kappa_{x, P M L}^{R} \tilde{d}_{P M L}^{R}\right) & \frac{j \sin \left(\kappa_{x, P M L}^{R} \tilde{d}_{P M L}^{R}\right)}{\kappa_{x, P M L}^{R}} \\
j_{\kappa_{x, P M L}^{R} \sin \left(\kappa_{x, P M L}^{R} \tilde{d}_{P M L}^{R}\right)}^{\cos \left(\kappa_{x, P M L}^{R} \tilde{d}_{P M L}^{R}\right)} \mid
\end{array}\right|\left|\begin{array}{l}
U_{R} \\
V_{R}
\end{array}\right|
\end{gathered}
$$

According to the governing equation of 2-D waveguide for TE polarization, $\mathrm{U}$ and $\mathrm{V}$ are proportional to $E_{y}$ and $H_{z}$, respectively. So $\mathrm{U}$ and $\mathrm{V}$ are continuous at the 
junctions. Therefore the transfer matrix for the whole cross-section can be written through the layer-by-layer multiplication of transfer matrix for each layer.

$$
\begin{gathered}
\left|\begin{array}{l}
U_{L} \\
V_{L}
\end{array}\right|=T_{\text {total }}\left|\begin{array}{l}
U_{R} \\
V_{R}
\end{array}\right|=\left|\begin{array}{cc}
T_{11}^{\text {total }} & T_{12}^{\text {total }} \\
T_{21}^{\text {total }} & T_{22}^{\text {total }}
\end{array}\right|\left|\begin{array}{c}
U_{R} \\
V_{R}
\end{array}\right| \\
T_{\text {total }}=T_{P M L}^{L} T_{1} T_{2} \ldots \ldots . T_{m} \cdots \cdots T_{N} T_{P M L}^{R}
\end{gathered}
$$

In TM polarization case, we define two variables, $U=A^{+}+A^{-}$, $V=\frac{\kappa_{x}}{n^{2}}\left(A^{+}-A^{-}\right), n$ is the local index. $\mathrm{U}$ and $\mathrm{V}$ are proportional to $H_{y}$ and $E_{z}$ which are continuous at the junctions. In the similar way for TM polarization

$$
\begin{aligned}
& T_{m}=\left|\begin{array}{cc}
\cos \left(\kappa_{x, m} d_{m}\right) & \frac{j n_{m}^{2} \sin \left(\kappa_{x, m} d_{m}\right)}{\kappa_{x, m}} \\
\frac{j \kappa_{x, m} \sin \left(\kappa_{x, m} d_{m}\right)}{n_{m}^{2}} & \cos \left(\kappa_{x, m} d_{m}\right)
\end{array}\right| \\
& \left|\begin{array}{l}
U_{L} \\
V_{L}
\end{array}\right|=T_{P M L}^{L}\left|\begin{array}{l}
U_{1} \\
V_{1}
\end{array}\right|=\left|\begin{array}{cc}
\cos \left(\kappa_{x, P M L}^{L} \tilde{d}_{P M L}^{L}\right) & \frac{j n_{P M L, L}^{2} \sin \left(\kappa_{x, P M L}^{L} \tilde{d}_{P M L}^{L}\right)}{\kappa_{x, P M L}^{L}} \\
\frac{j \kappa_{x, P M L}^{L} \sin \left(\kappa_{x, P M L}^{L} \tilde{d}_{P M L}^{L}\right)}{n_{P M L, L}^{2}} & \cos \left(\kappa_{x, P M L}^{L} \tilde{d}_{P M L}^{L}\right)
\end{array}\right|\left|\begin{array}{l}
U_{1} \\
V_{1}
\end{array}\right| \\
& \left|\begin{array}{l}
U_{N+1} \\
V_{N+1}
\end{array}\right|=T_{P M L}^{R}\left|\begin{array}{l}
U_{R} \\
V_{R}
\end{array}\right|=\left|\begin{array}{cc}
\cos \left(\kappa_{x, P M L}^{R} \tilde{d}_{P M L}^{R}\right) & \frac{j n_{P M L, R}^{2} \sin \left(\kappa_{x, P M L}^{R} \tilde{d}_{P M L}^{R}\right)}{\kappa_{x, P M L}^{R}} \\
\frac{j \kappa_{x, P M L}^{R} \sin \left(\kappa_{x, P M L}^{R} \tilde{d}_{P M L}^{R}\right)}{n_{P M L, R}^{2}} & \cos \left(\kappa_{x, P M L}^{R} \tilde{d}_{P M L}^{R}\right)
\end{array}\right|\left|\begin{array}{l}
U_{R} \\
V_{R}
\end{array}\right| \\
& T_{\text {total }}=T_{P M L}^{L} T_{1} T_{2} \cdots \cdots T_{m} \cdots \cdots T_{N} T_{P M L}^{R}=\left|\begin{array}{ll}
T_{11}^{\text {total }} & T_{12}^{\text {total }} \\
T_{21}^{\text {total }} & T_{22}^{\text {total }}
\end{array}\right|
\end{aligned}
$$

At PRB, $U_{L}=0, U_{R}=0$, therefore we get the implicit characteristics equation for both TE and TM polarization cases

$$
f\left(\beta^{2}, R_{P M L}\right)=T_{21}^{\text {total }}=0
$$


When $R_{P M L}$ is 1 , the roots of (4.24) fall into two parts: the real ones and imaginary ones, which are corresponding to the guided and evanescent modes. As mentioned above, the mode spacing of box modes strongly depend on the computation window size. The root locus moves from real and imaginary axis to complex plane, as $R_{P M L}$ changes from 1 to a small enough value. Figure 4.2 shows the root searching process of complex STM, in which q is an arbitrary small number, ' $k$ ' denotes the $k^{\text {th }}$ step of iteration.

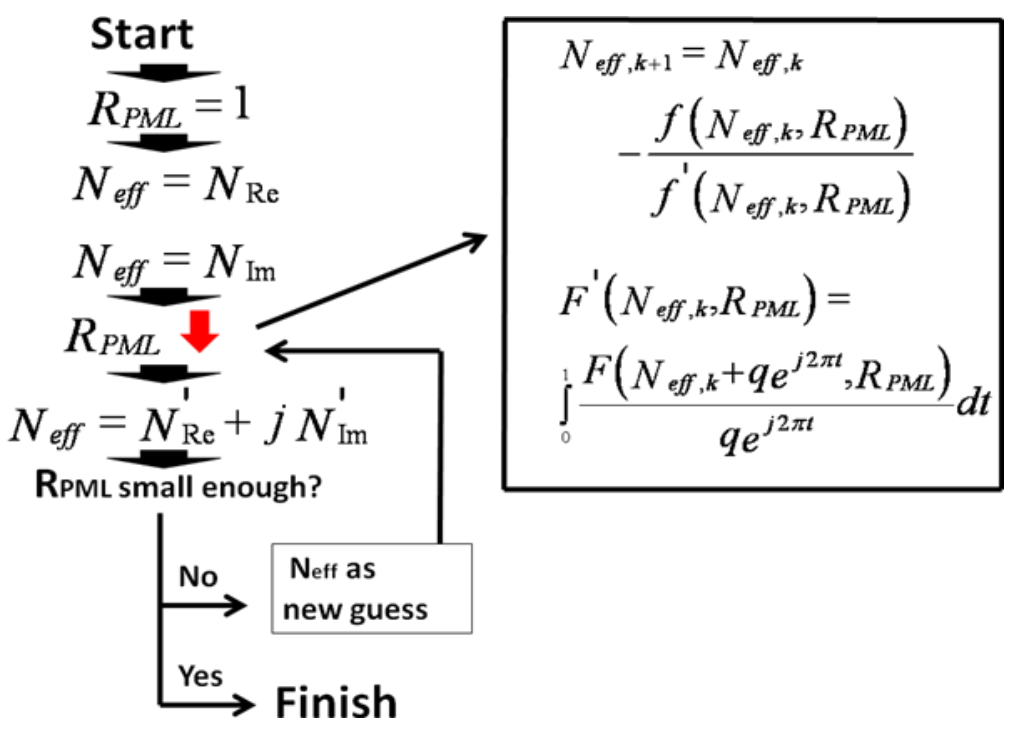

Figure 4.2. Methodology of Complex STM

\subsection{Validation of Complex STM}

\subsection{Slab Waveguide}

We consider a symmetric slab waveguide (we call it slab waveguide 1) in Figure 2.4(b), with $\mathrm{n} 1=3.3, \mathrm{n} 2=3.17,2 \mathrm{~S}=0.2 \mu \mathrm{m}, \mathrm{dPML}=1 \mu \mathrm{m}, 2 \mathrm{~L}=4.2 \mu \mathrm{m}$, the wavelength is $1.55 \mu \mathrm{m}$. The mesh size for FD mode solver is $5 \mathrm{~nm}$. Figure 4.3 compares the mode 
spectrums at $R_{P M L}=1 e-2$ obtained from complex STM and HOFD scheme, respectively. We can see the coincidence is good. Figure 4.3 also shows the initial box modes at $R_{P M L}=1$.

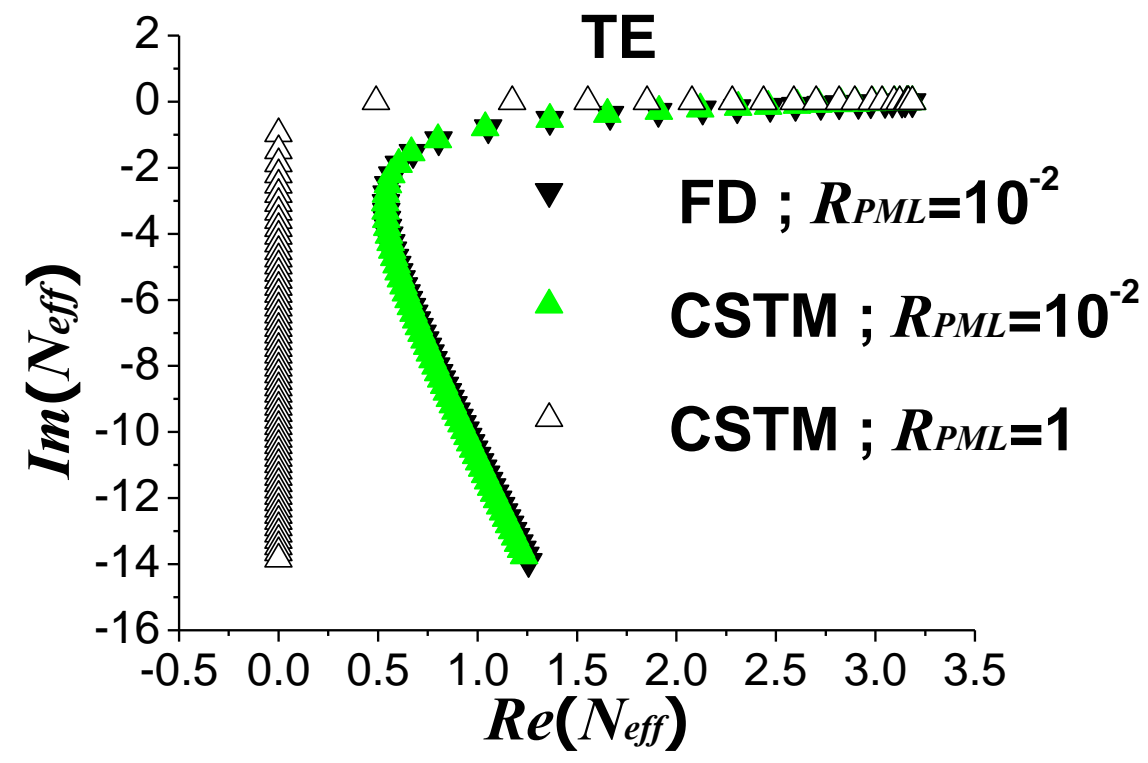

(a)

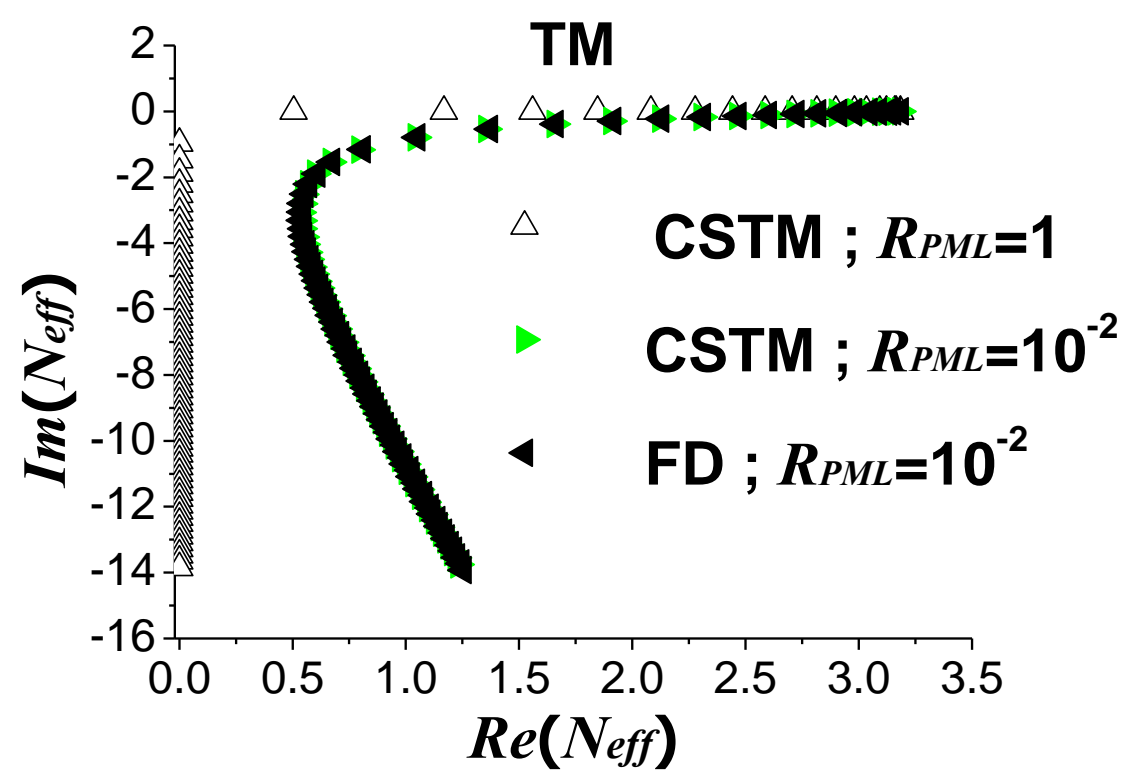

(b)

Figure 4.3. Mode spectrums of (a) TE and (b) TM cases in slab waveguide 1 
In order to further validate complex STM in slab waveguide, Figure 4.4 compares the modal field patterns with those of HOFD scheme for TE and TM polarizations. We further validate the complex mode orthogonality obtained from complex STM by plotting the modal field overlaps in Figure 4.5. We observe that the overlap integrals between different eigenmodes are zero so that the eigenmodes are orthogonal. Also, the self-overlaps for high-order complex modes are also close to be zero. 


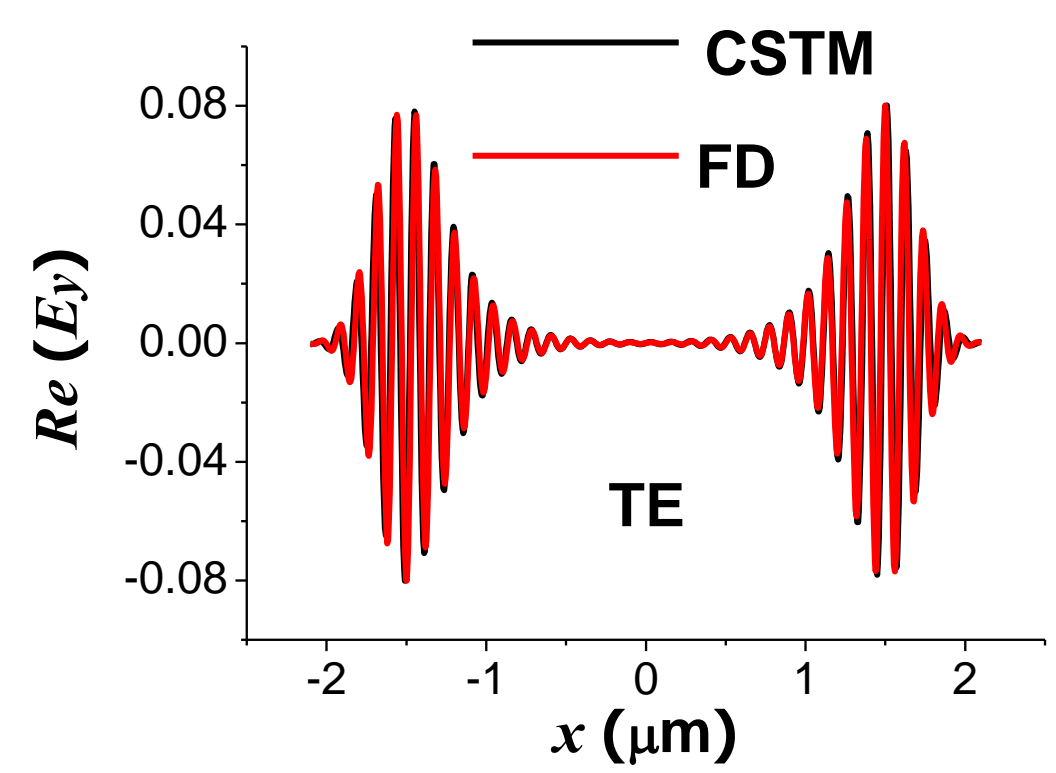

(a)

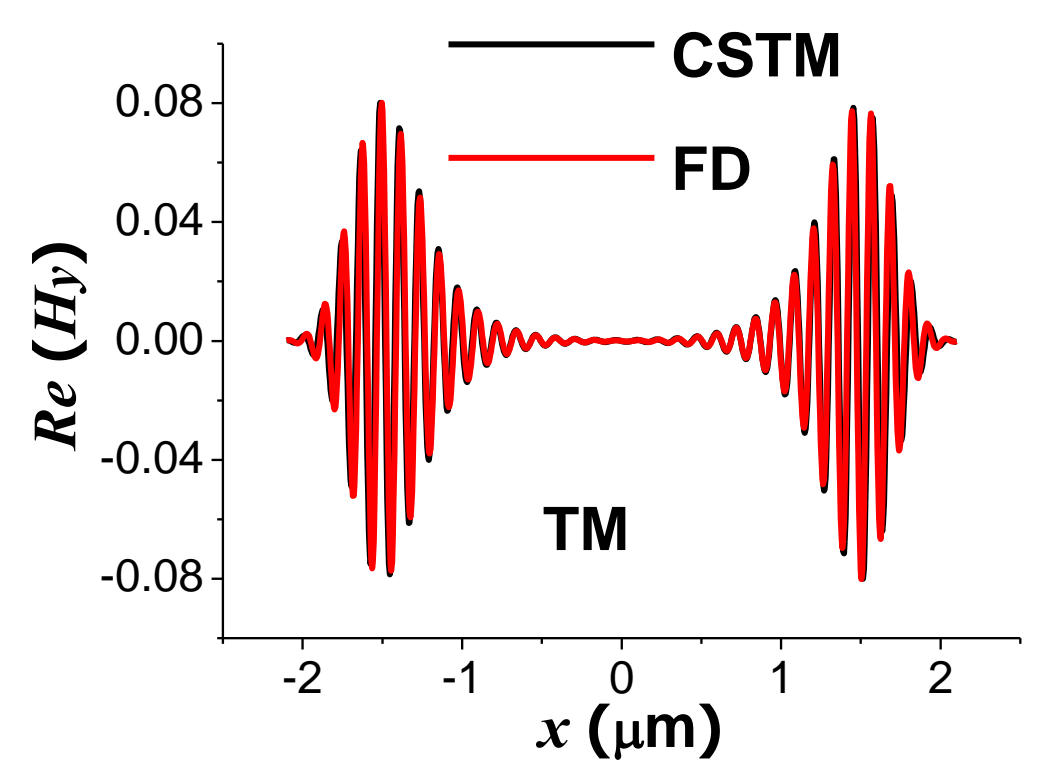

(b)

Figure 4.4. Modal field patterns in slab waveguide 1 (a) TE case. $N_{\text {eff }}$ for STM is $1.1412-12.4123 \mathrm{i}, \quad N_{\text {eff }}$ for FD is $1.1346-12.4096 \mathrm{i}(\mathrm{b}) \mathrm{TM}$ case. $N_{\text {eff }}$ for STM is $1.1468-12.4173 \mathrm{i}, \quad N_{e f f}$ for FD is $1.1378-12.4149 \mathrm{i}$ 


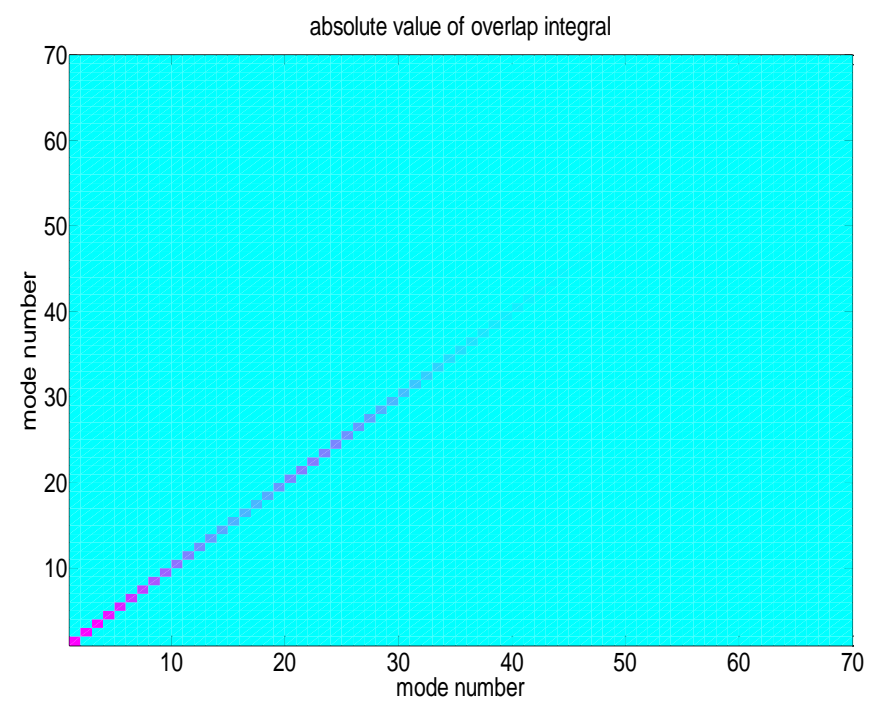

(a)

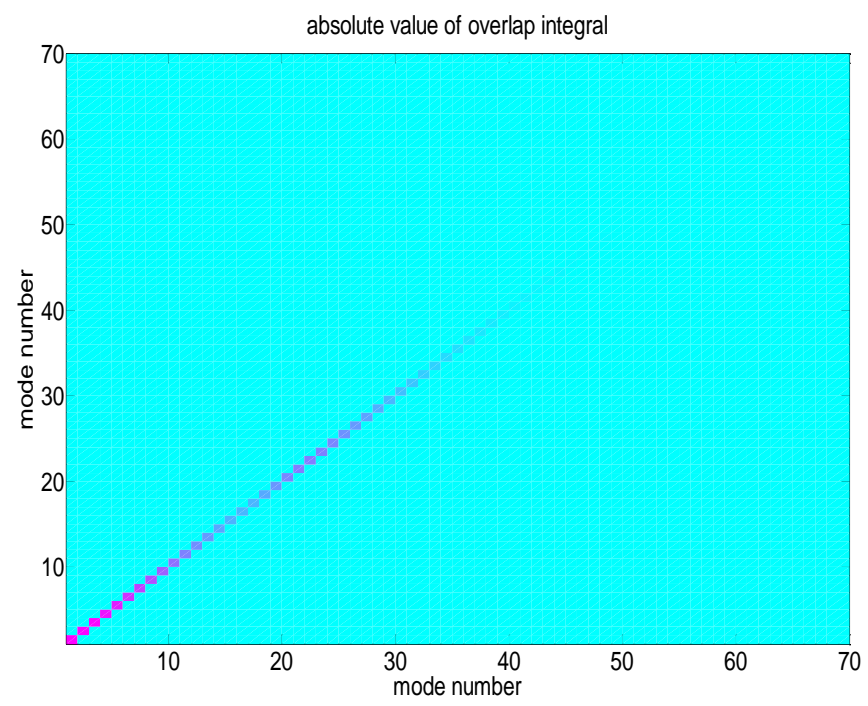

(b)

Figure 4.5. Mode orthogonality for complex STM in slab waveguide 1. (a) TE case (b) TM case

We further consider another slab waveguide (we call it slab waveguide 2) in Figure $2.4(\mathrm{~b})$ with $\mathrm{n} 1=3.3, \mathrm{n} 2=3.17,2 \mathrm{~S}=0.3 \mu \mathrm{m}, \quad \mathrm{dPML}=1 \mu \mathrm{m}, \quad 2 \mathrm{~L}=8.3 \mu \mathrm{m}$, the wavelength is $1.55 \mu \mathrm{m}$. The mesh size for FD mode solver is $5 \mathrm{~nm}$. Figure 4.6 compares the mode spectrums at $R_{P M L}=1 e-3$ obtained from complex STM and HOFD scheme, respectively. We can see the coincidence is good. 


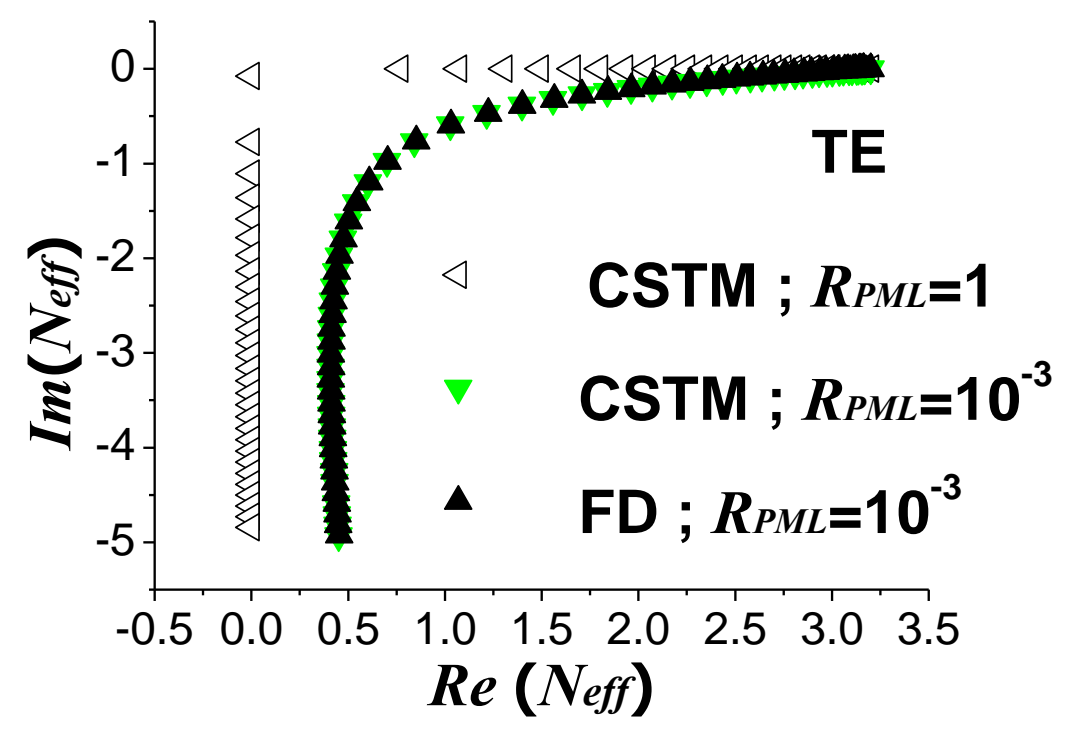

(a)

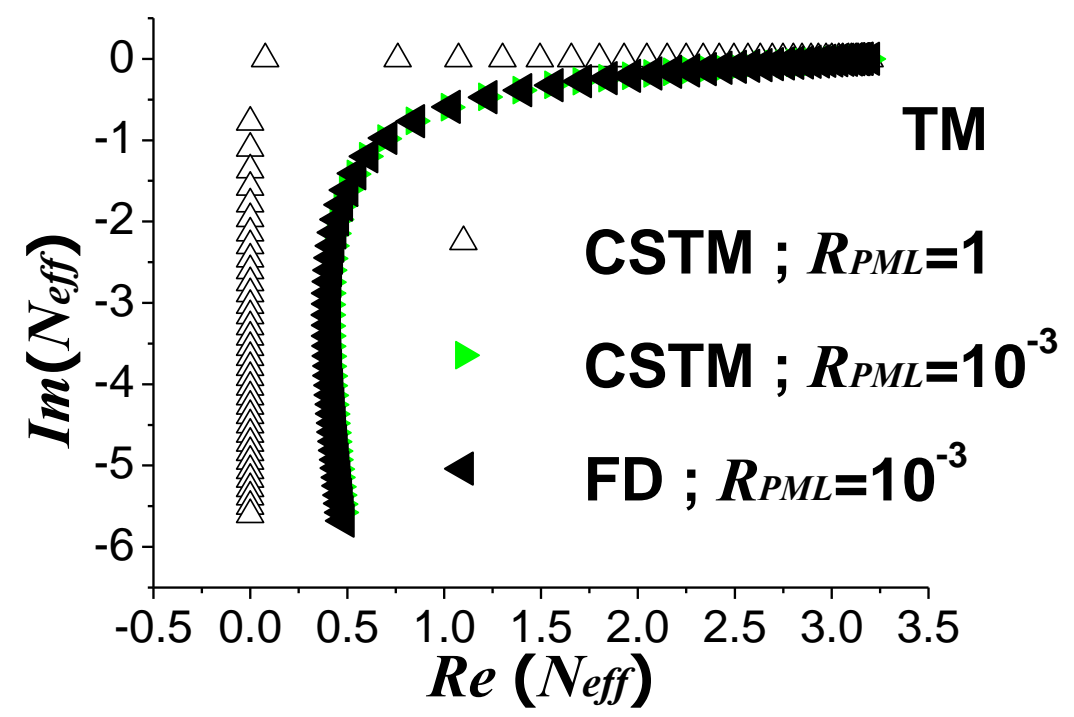

(b)

Figure 4.6. Mode spectrums of (a) TE and (b) TM case in slab waveguide 2

Figure 4.7 describes the comparisons of modal patterns from complex STM and HOFD in both TE and TM cases. 


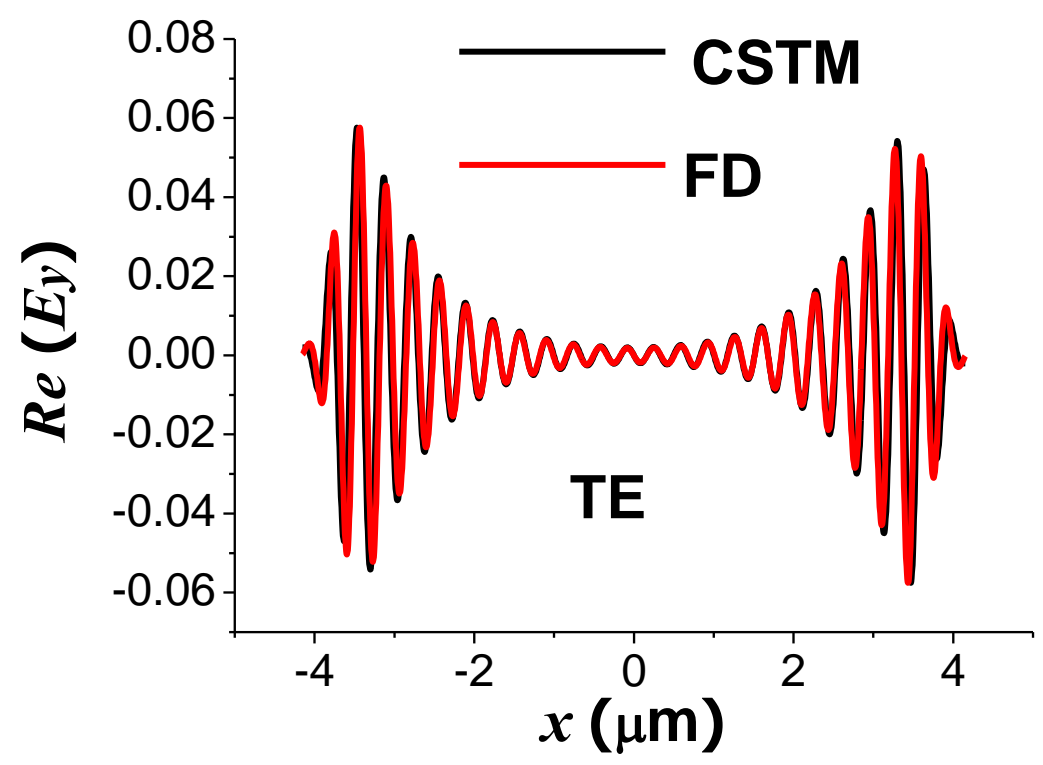

(a)

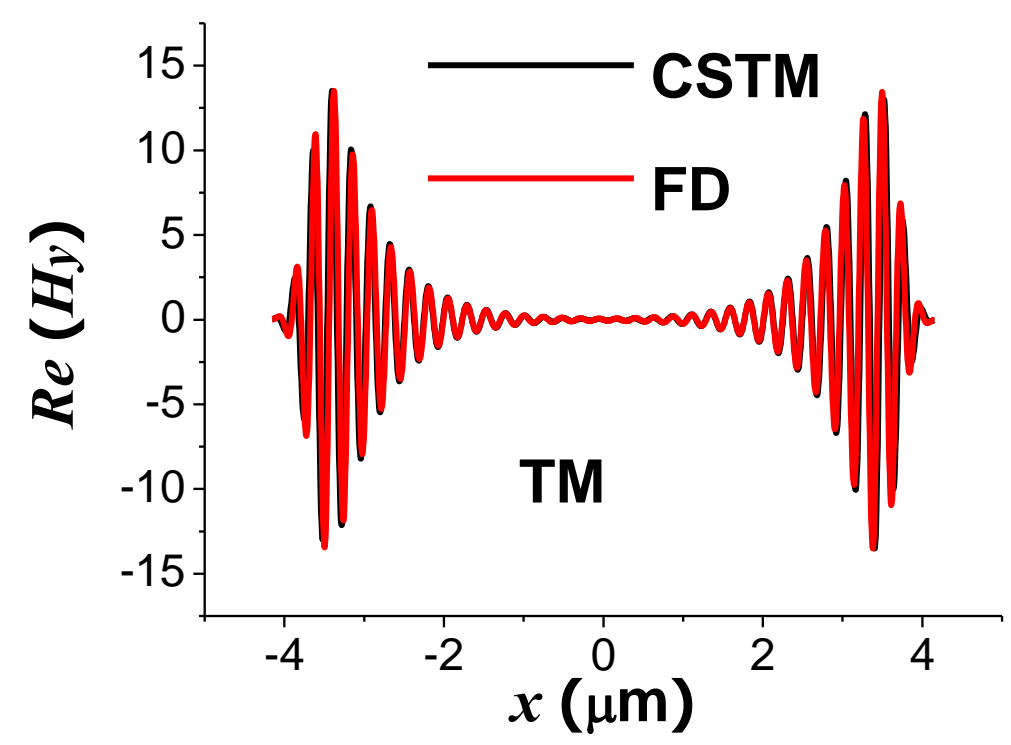

(b)

Figure 4.7. Modal field patterns of slab waveguide 2 (a) TE. $N_{\text {eff }}$ for complex STM is $0.4099-3.2799 \mathrm{i}, \quad N_{\text {eff }}$ for FD is $0.4135-3.4035 \mathrm{i}$ (b) TM. $N_{\text {eff }}$ for complex STM is $0.4775-5.5776 \mathrm{i}, \quad N_{\text {eff }}$ for FD is $0.4854-5.6794 \mathrm{i}$. 
Figure 4.8 validates the mode orthogonality of Complex STM for the slab waveguide 2.

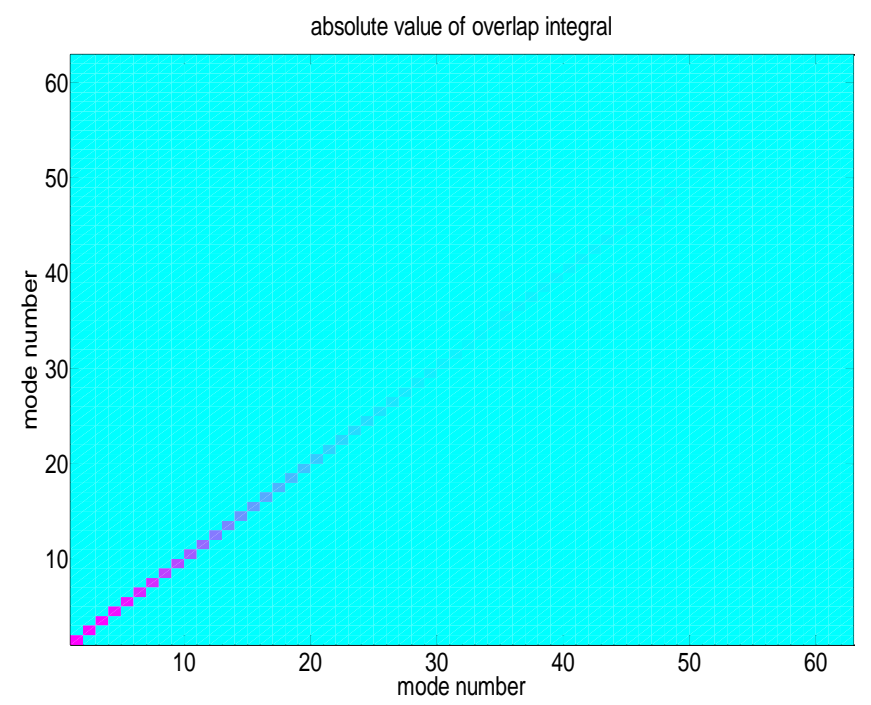

(a)

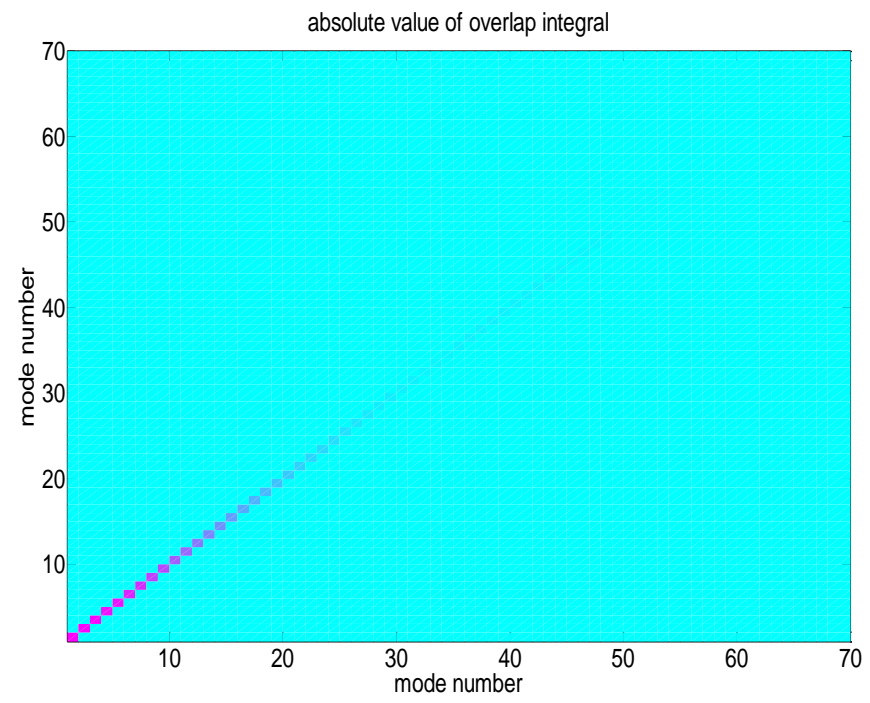

(b)

Figure 4.8. Mode orthogonality of complex STM in slab waveguide 2 (a) TE case (b) TM case

\subsection{Hollow Waveguide}


We consider the hollow waveguide in Figure 2.10, with dair $=1.5 \mu \mathrm{m}$, dPML $=5 \mu \mathrm{m}$.

Figure 4.9 validates the mode spectrums from complex STM with those of FD in hollow waveguide in both TE and TM cases. The mesh size for FD solver is $20 \mathrm{~nm}$.

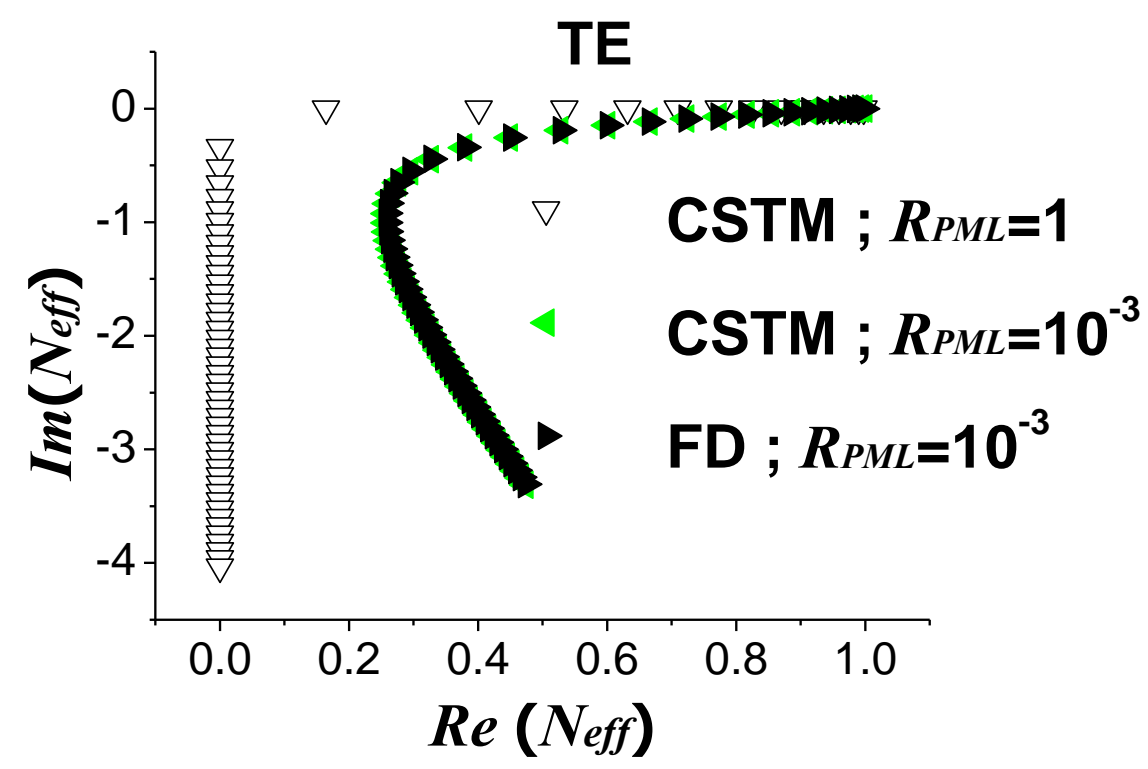

(a)

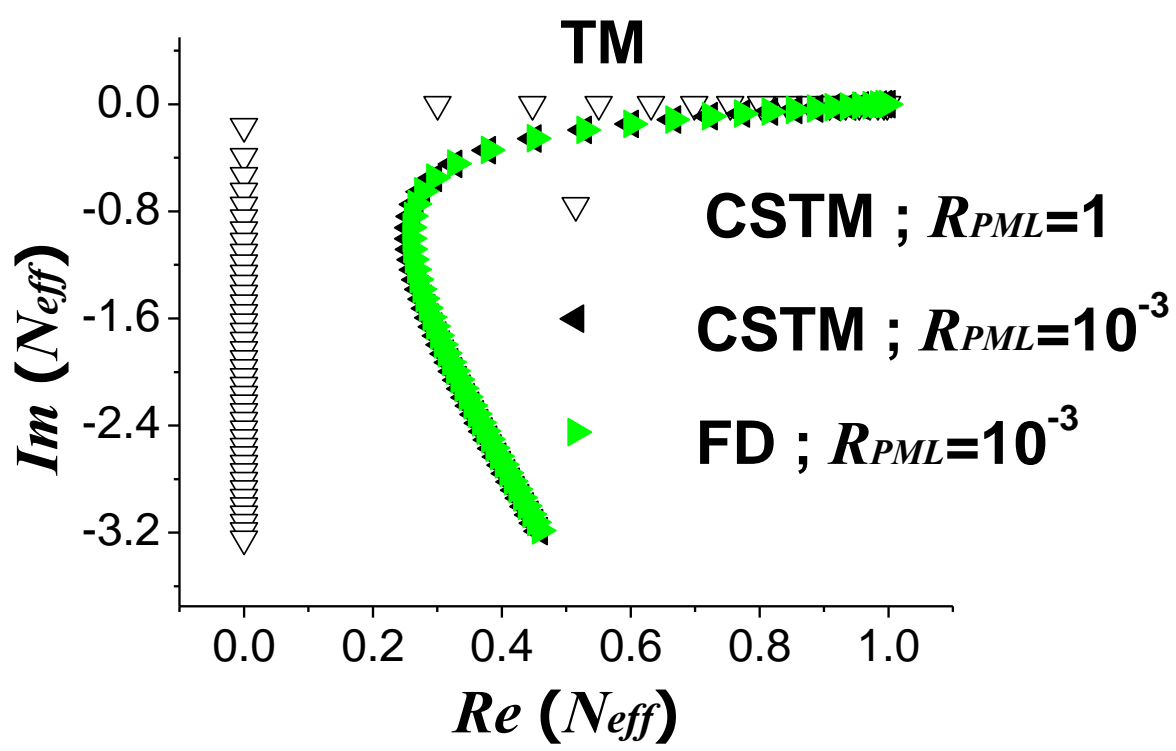

(b)

Figure 4.9. Mode spectrums of hollow waveguide (a) TE case (b) TM case 


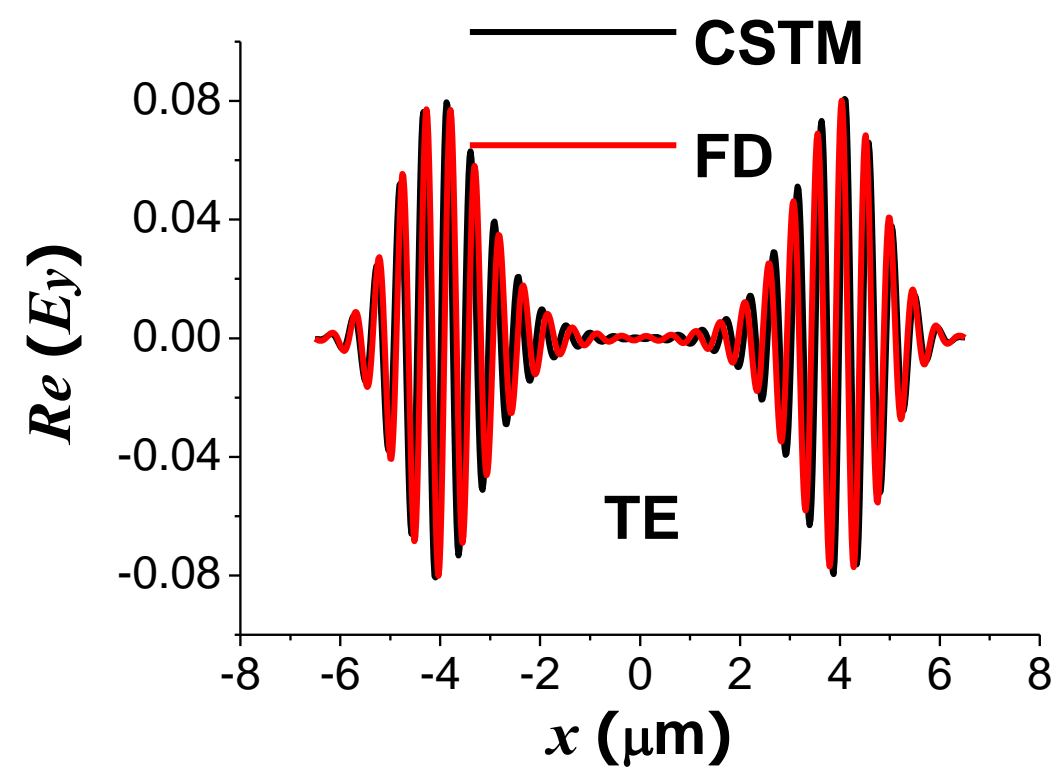

(a)

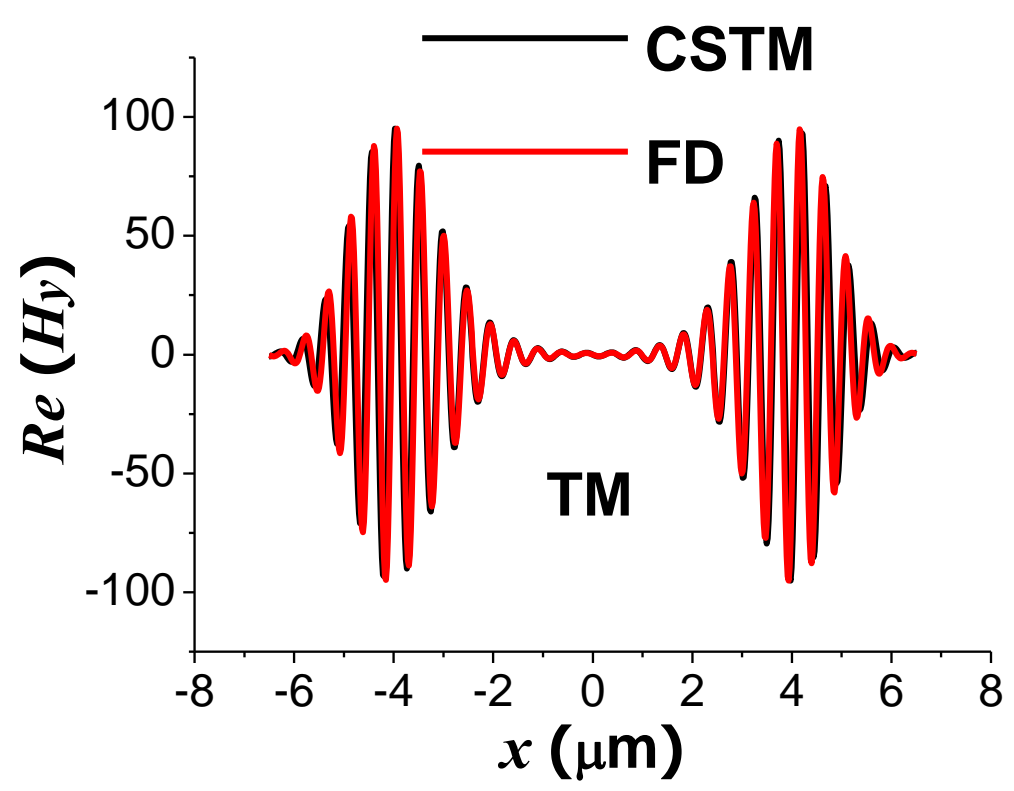

(b)

Figure 4.10. Modal field patterns of hollow waveguide (a) TE. $N_{\text {eff }}$ for complex STM is $0.4099-3.2797 \mathrm{i}, \quad N_{\text {eff }}$ for FD is $0.4135-3.4035 \mathrm{i}$ (b) TM. $N_{\text {eff }}$ for complex STM is $0.4440-3.0675 \mathrm{i}, \quad N_{\text {eff }}$ for FD is $0.4526-3.1232 \mathrm{i}$ 
Figure 4.10 compares the modal patterns from complex STM with those from FD in TE and TM cases. Figure 4.11 validates the mode orthogonality of complex STM in hollow waveguide in TE and TM cases

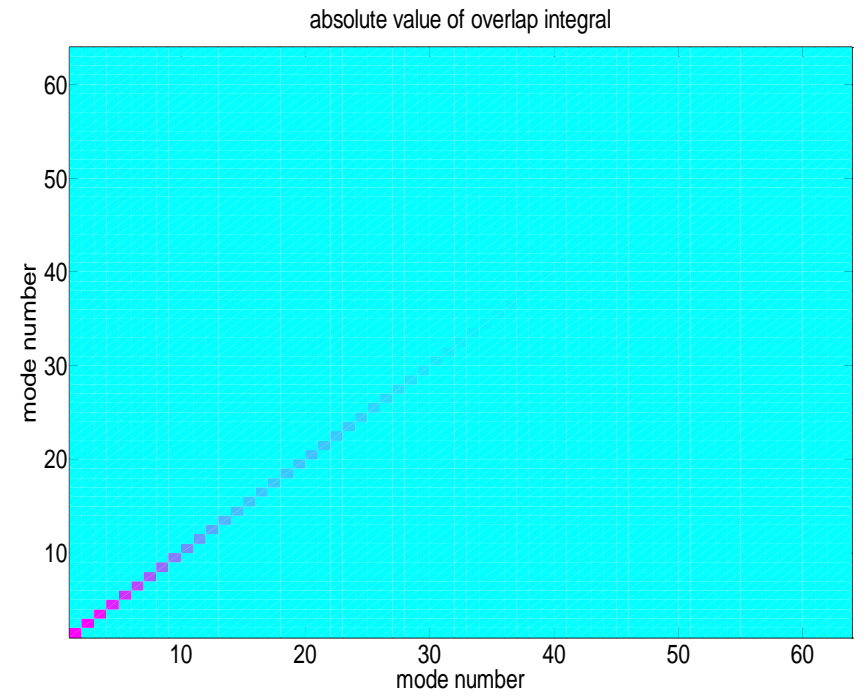

(a)

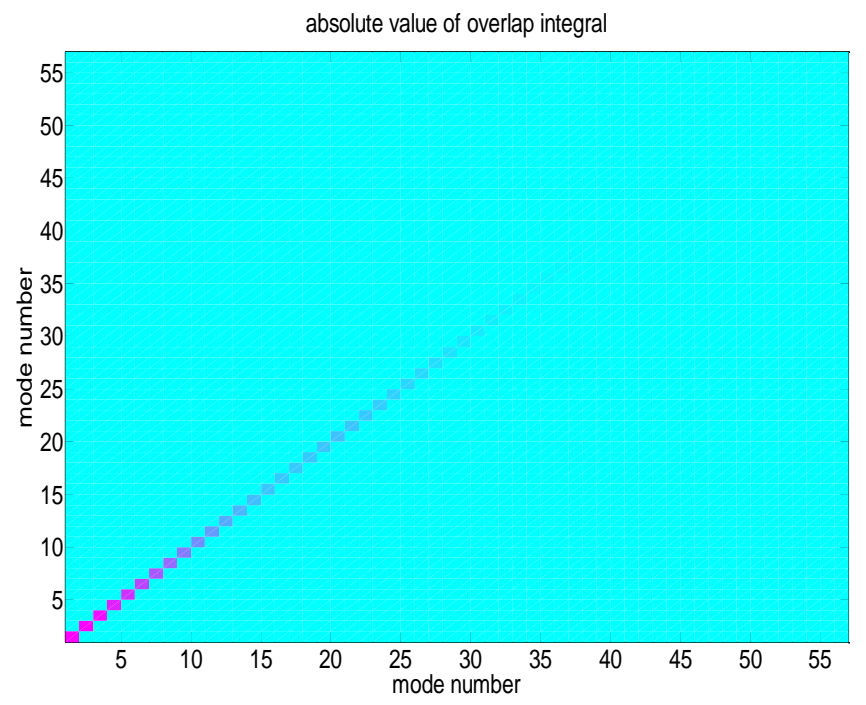

(b)

Figure 4.11. Mode orthogonality of complex STM in hollow waveguide (a) TE case (b) TM case 


\subsection{Leaky Waveguide}

We study a 2-D leaky waveguide shown in Figure 4.12. The wavelength is $1.55 \mu \mathrm{m}$, and the mesh size for FD solver is $5 \mathrm{~nm}$. Figure 4.13 compares the mode spectrums from complex STM and FD for the leaky waveguide in TE and TM cases.

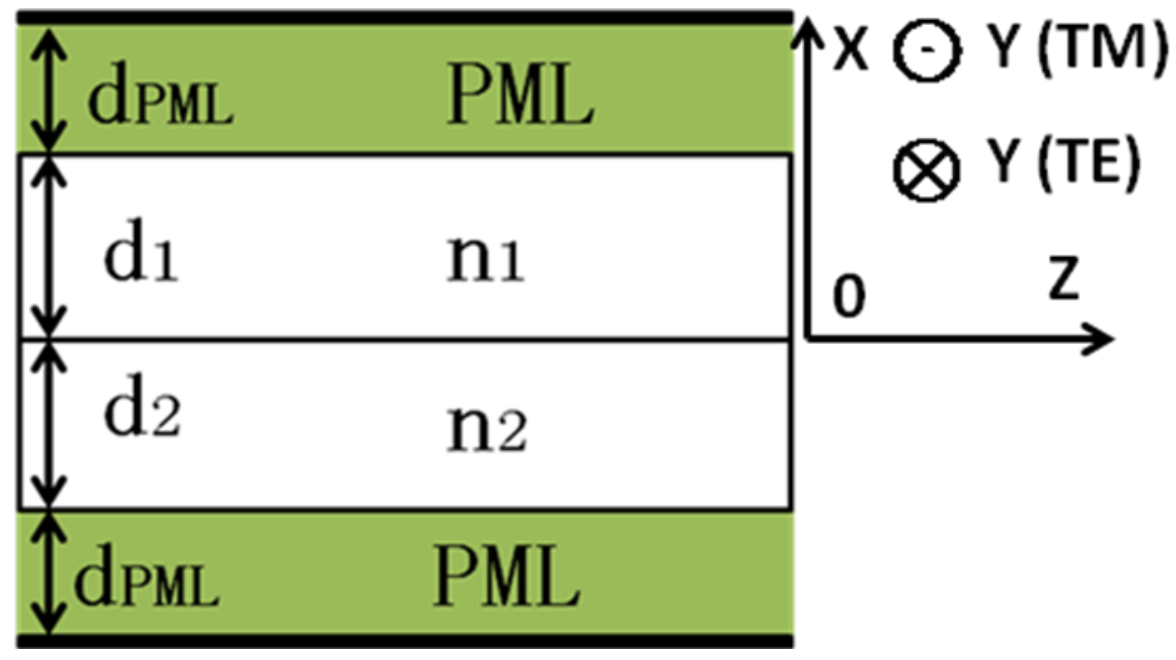

Figure 4.12. The schematic of a 2 -D leaky waveguide. $\mathrm{n} 1=3.17, \mathrm{n} 2=3.3, \mathrm{~d} 1=2 \mu \mathrm{m}$, $\mathrm{d} 2=2 \mu \mathrm{m}, \mathrm{dPML}=1 \mu \mathrm{m}$. 


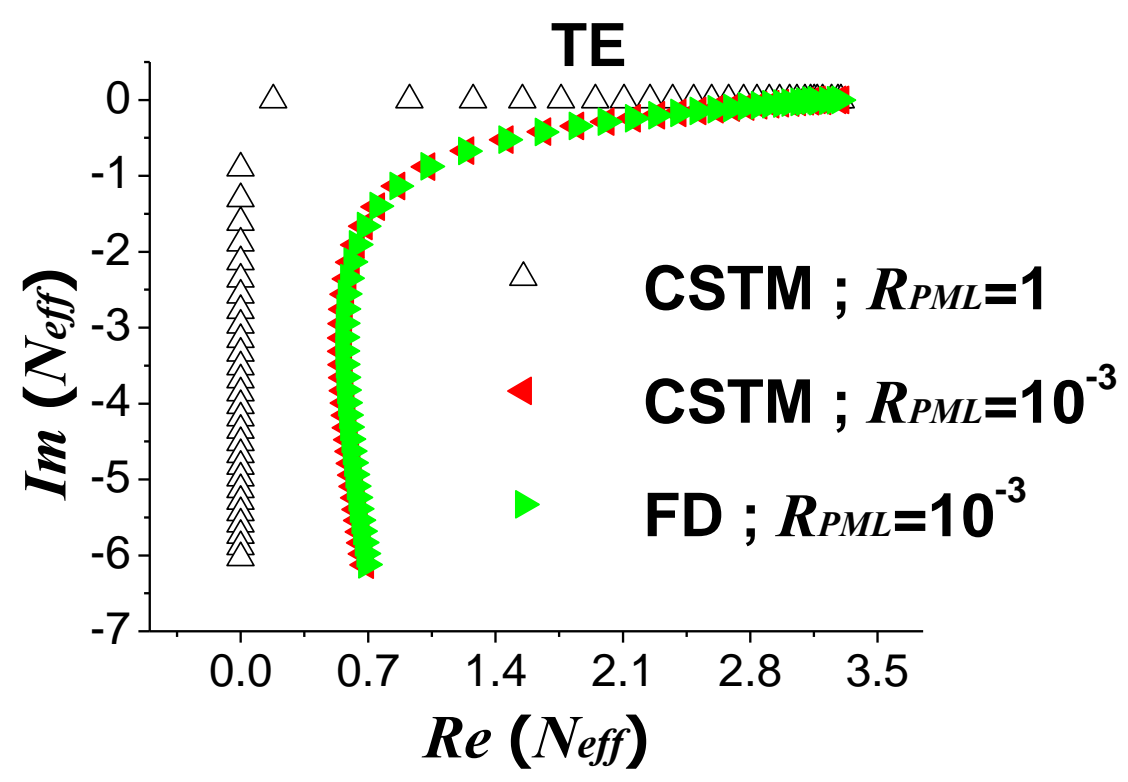

(a)

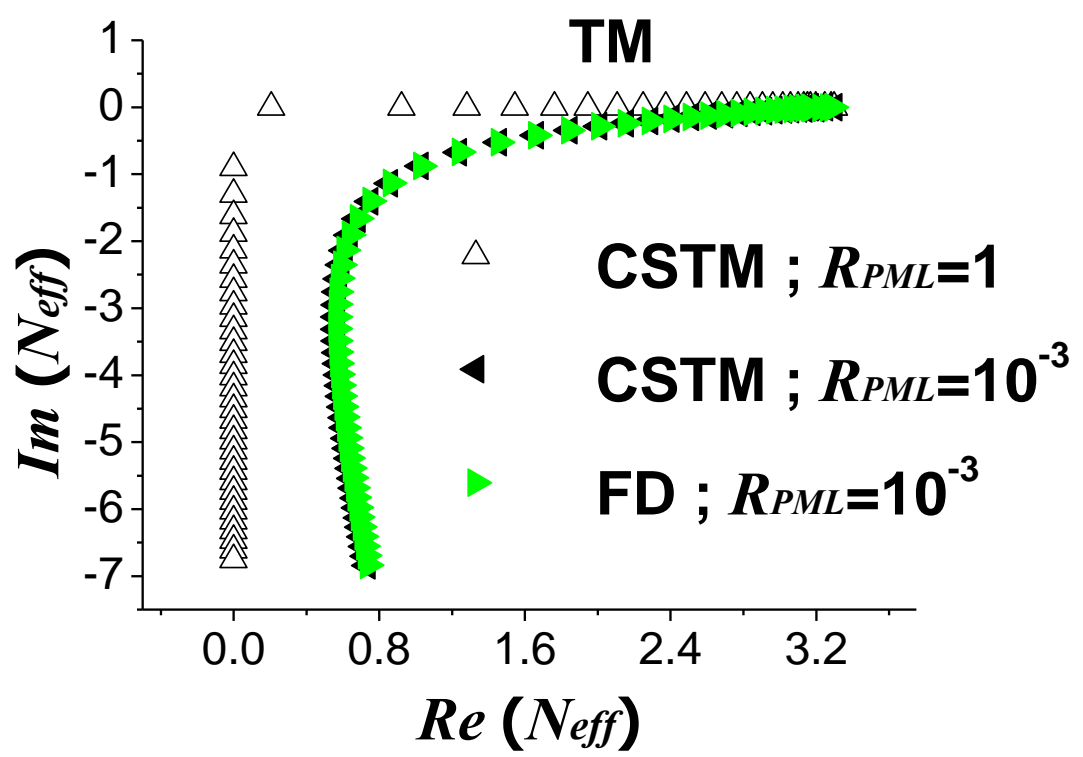

(b)

Figure 4.13. Mode spectrums of (a) TE and (b) TM cases in leaky waveguide

Figure 4.14 compares the modal field patterns from complex STM and HOFD in leaky waveguide in TE and TM cases. 


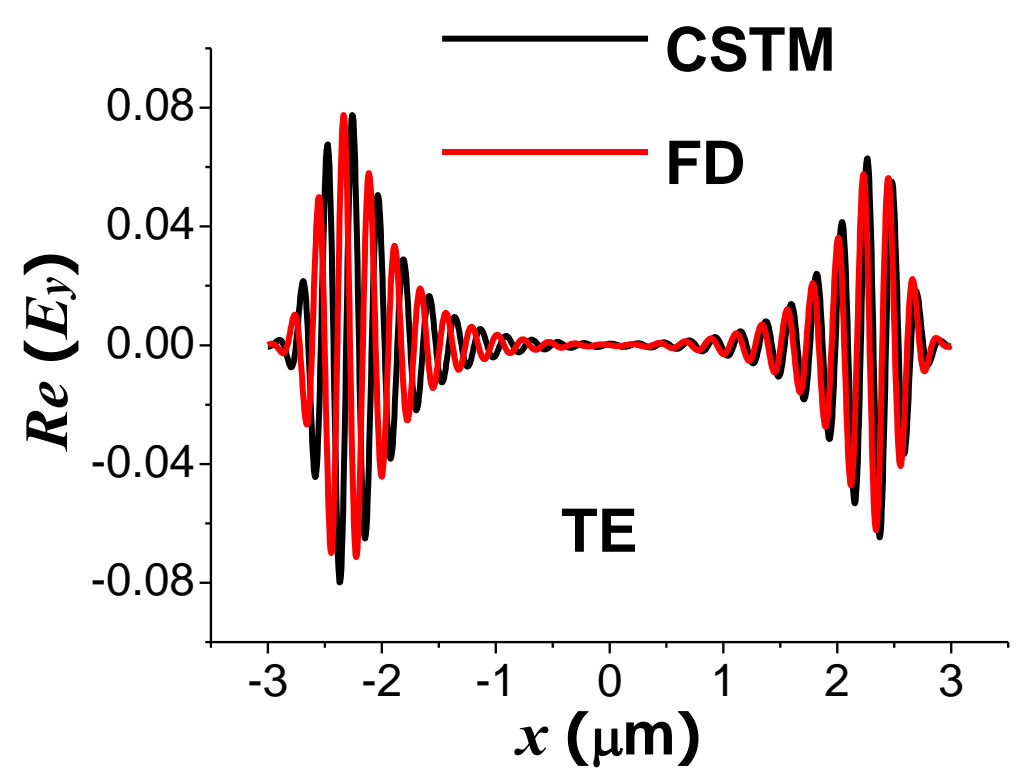

(a)

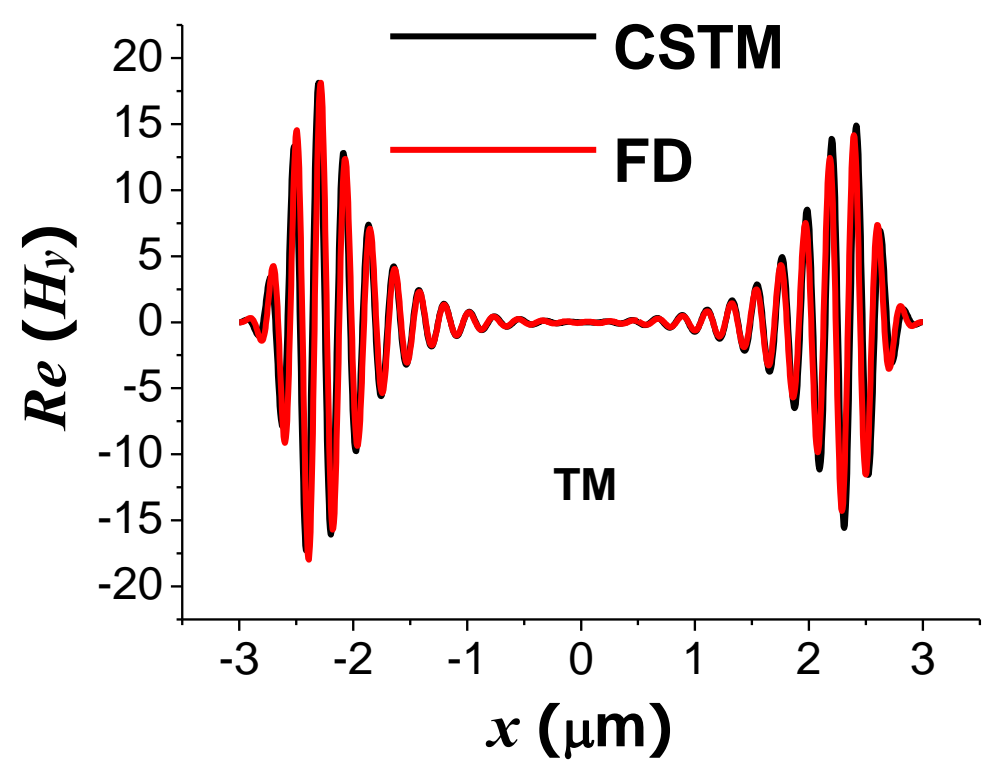

(b)

Figure 4.14. Modal field patterns of leaky waveguide (a) TE. $N_{\text {eff }}$ for STM is 0.6867

- 6.1270i, $\quad N_{\text {eff }}$ for FD is 0.6907 - 6.1201i. (b) TM. $\quad N_{\text {eff }}$ for STM is $0.6956-6.2705 \mathrm{i}$, $N_{\text {eff }}$ for FD is $0.7092-6.4068 \mathrm{i}$ 
Figure 4.15 validates the mode orthogonality of complex STM in leaky waveguide in

TE and TM cases.

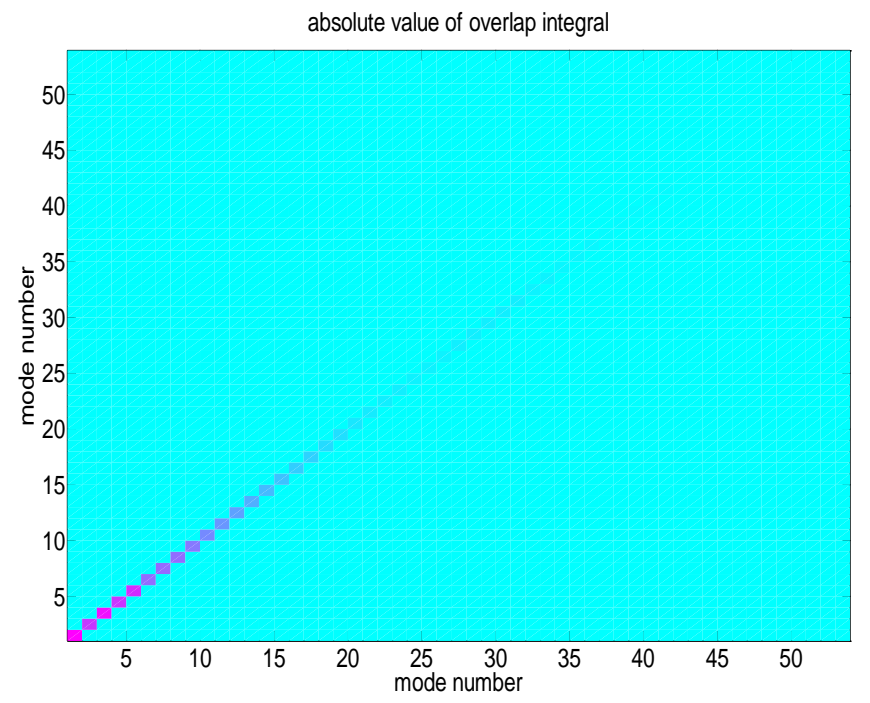

(a)

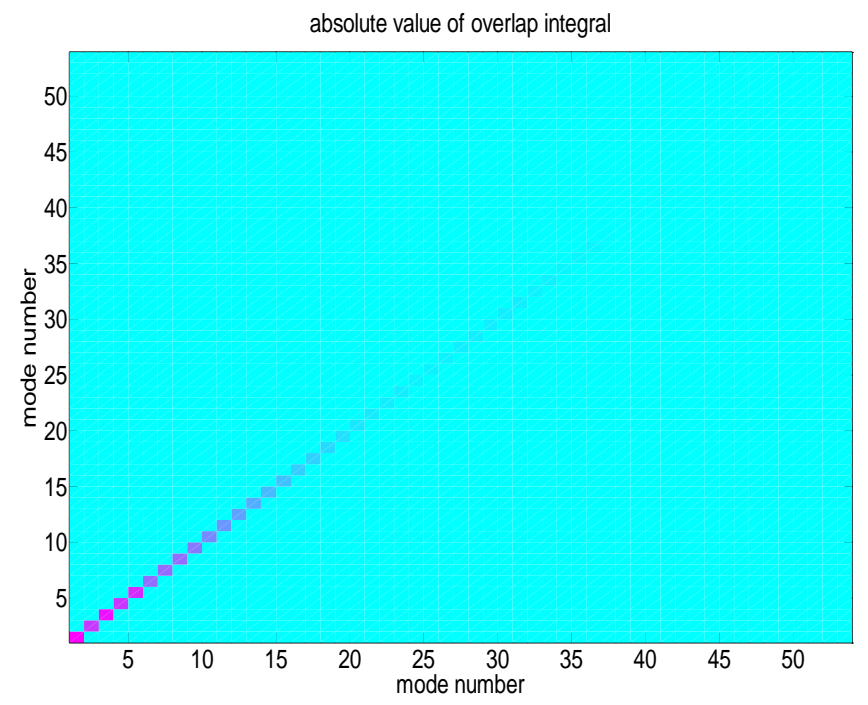

(b)

Figure 4.15. Mode orthogonality of complex STM in leaky waveguide (a) TE case (b) TM case 


\section{Chapter 5}

\section{Power conservation of Complex Mode Matching Method (CMMM)}

\subsection{Introduction}

Once the waveguide is enclosed with PRB and PML, the mode spectrum is composed of guided modes and complex modes. The power flow of guided modes is along longitudinal direction, while for complex modes power flows not only longitudinally but also transversely. For an arbitrary enclosed space in waveguide region without any material absorption, the power flowing into and that out of such a space should be balanced. However, until now this conception of power conservation for complex mode matching method has not been clearly proved.

In this chapter, we analytically prove the power conservation in the framework of complex mode matching method in hollow waveguide, and numerically validate it in leaky waveguide and waveguide discontinuity.

\subsection{Analytical Validation in Hollow Waveguide}

Figure 5.1 describes a hollow waveguide terminated with PML and PRB. A rectangular box is located in its waveguide region. The sides above and below of such a rectangular box is just at the starting position of PML above and below. $\hat{X}$ and 
$\tilde{Z}$ are the transverse and longitudinal directions, respectively. The coordinates of the four vertexes of this rectangular box in the $X-Z$ plane are $\left(-d_{\text {air }}, Z_{1}\right),\left(-d_{\text {air }}, Z_{2}\right),\left(d_{\text {air }}, Z_{1}\right)$ and $\left(d_{\text {air }}, Z_{2}\right)$, respectively. The power flowing into the box from the left side is noted as $P_{z, \text { in }} \cdot P_{z, \text { out }}, P_{x, u p}$ and $P_{x, \text { down }}$ represent the power flow through the right side, the side above and that below, respectively.

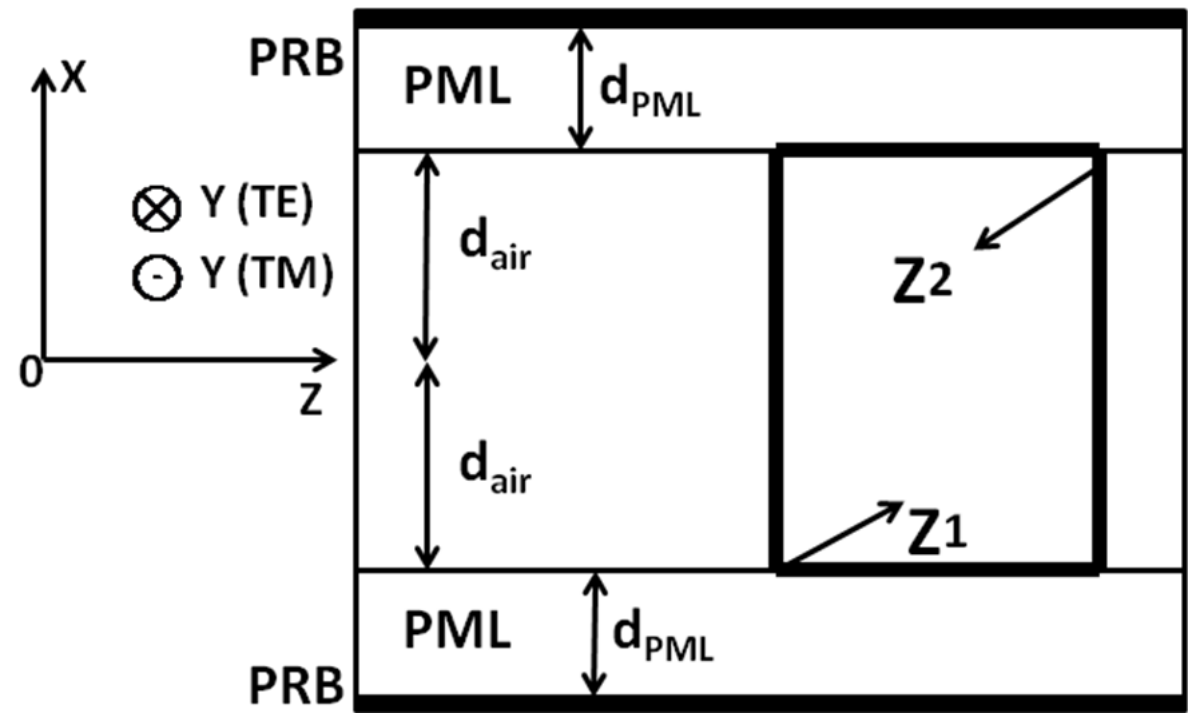

Figure 5.1. The schematic of a 2-D hollow waveguide with a rectangular passive box in waveguide region

The propagation constant and transverse wave vector are complex, so we write the expressions as

$$
\begin{gathered}
\beta_{n}=\beta_{n R}-j \beta_{n I} \\
\kappa_{x, n}=\kappa_{x R, n}-j \kappa_{x I, n}
\end{gathered}
$$

Accordingly the analytical modal field pattern in polarization 


$$
\begin{gathered}
P_{z, \text { in }}=\frac{1}{2} \operatorname{Re}\left(\int_{- \text {dair }}^{\text {dair }} E_{y} \bullet H_{x}^{*} d x\right) \\
=\frac{-1}{8 \omega \mu_{0}}\left[\frac{\beta_{R, n}}{\kappa_{x I, n}}\left(e^{2 \kappa_{x l, n} d_{\text {air }}}-e^{-2_{x x l, n} d_{\text {air }}}\right)-\frac{2 \beta_{R, n}}{\kappa_{x R, n}} \sin \left(2 \kappa_{x R, n} d_{\text {air }}\right)\right] \\
P_{x, u p}=\operatorname{Re}\left(\int_{z 1}^{z 2} E_{y, x=d_{\text {dir }}} \bullet H_{z, x=d a i r}^{*} d z\right)=\frac{-1}{16 \omega \mu_{0}}\left[\frac{\kappa_{x R, n}}{\beta_{n I}}\left(e^{2 \kappa_{x l l, n} d_{\text {air }}}-e^{-2 \kappa_{x, n l} d_{\text {air }}}\right)\right. \\
\left.-2 \frac{\kappa_{x I, n}}{\beta_{n I}} \sin \left(2 \kappa_{x R, n} d_{\text {air }}\right)\right]\left[1-e^{-2 \beta_{n I}(z 2-z 1)}\right]
\end{gathered}
$$

Following the dispersion relationship $\beta_{n}^{2}+\kappa_{x, n}^{2}=k_{0}^{2}$, we obtain

$$
\kappa_{x R, n} \kappa_{x I, n}=-\beta_{n R} \beta_{n I}
$$

Since

$$
\begin{gathered}
P_{z, \text { out }}=e^{-2 \beta_{n l}\left(z_{2}-Z_{1}\right)} P_{z, \text { in }} \\
P_{x, \text { down }}=-P_{x, \text { up }}
\end{gathered}
$$

We obtain

$$
P_{z, \text { in }}-P_{z, \text { out }}=P_{x, \text { down }}-P_{x, \text { up }}
$$

Now the power conservation of CMMM in hollow waveguide has been proved, similarly we prove the relation is held in TM case

$$
P_{z, \text { in }}-P_{z, \text { out }}=P_{x, \text { down }}-P_{x, u p}
$$

\subsection{Numerical Validation}

\subsection{Leaky Waveguide}

For leaky waveguide, we didn't provide the analytical proof as the integral along longitudinal direction is much more complicated. Instead, we calculated the power flow in each direction numerically. Figure 5.2 shows a 2-D step-index leaky 
waveguide, with a rectangular box placed just in its waveguide region. The wavelength is $1.5 \mu \mathrm{m}$. We assume that a complex mode is launched along the longitudinal direction.

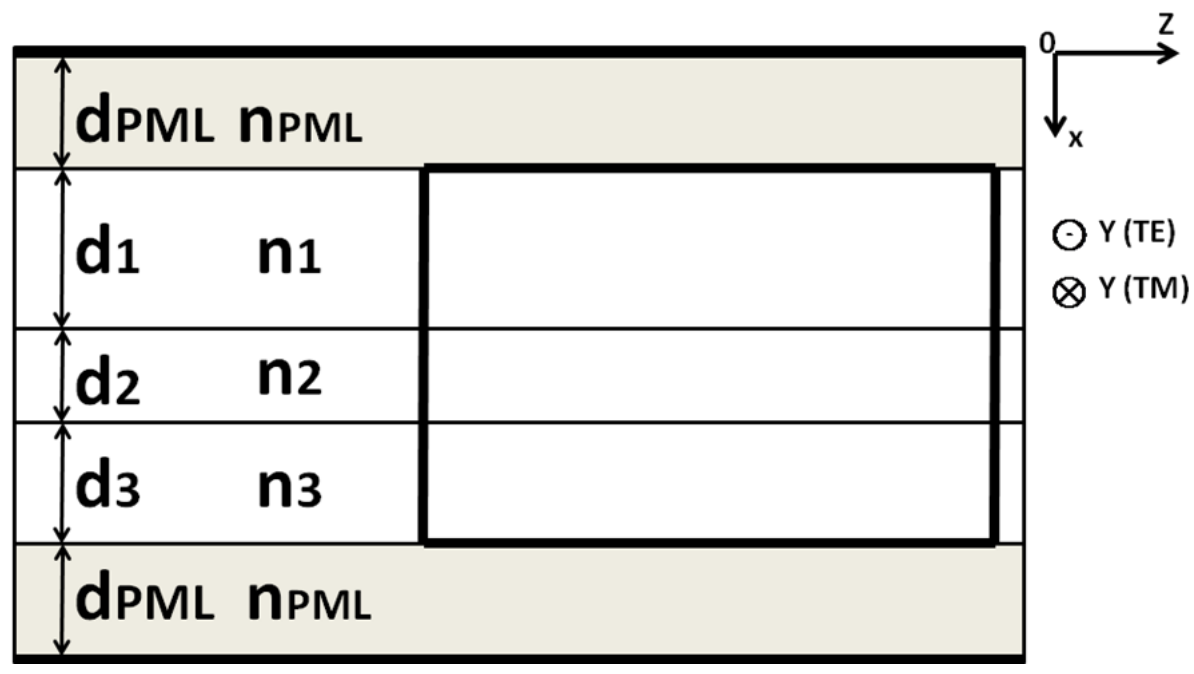

Figure 5.2. The schematic of a 2 -D step-index leaky waveguide.d1 $=4 \mu \mathrm{m}, \mathrm{d} 2=2 \mu \mathrm{m}$, $\mathrm{d} 3=3 \mu \mathrm{m}, \mathrm{n} 1=2.2, \mathrm{n} 2=2, \mathrm{n} 3=1, \mathrm{dPML}=3 \mu \mathrm{m}$

Figure 5.3(a) and Figure 5.4(a) show the modal field amplitude patterns of the launched mode obtained from HOFD scheme in TE and TM polarizations, respectively. The mesh size is $20 \mathrm{~nm}$. If $R_{P M L}$ is smaller, the waveguide region confinement factor is decreased, and the field oscillation in PML region is also suppressed. Figure 5.3(b) and Figure5.4(b) describe the convergence of $N_{\text {eff }}$ with reference to $R_{P M L}$. 


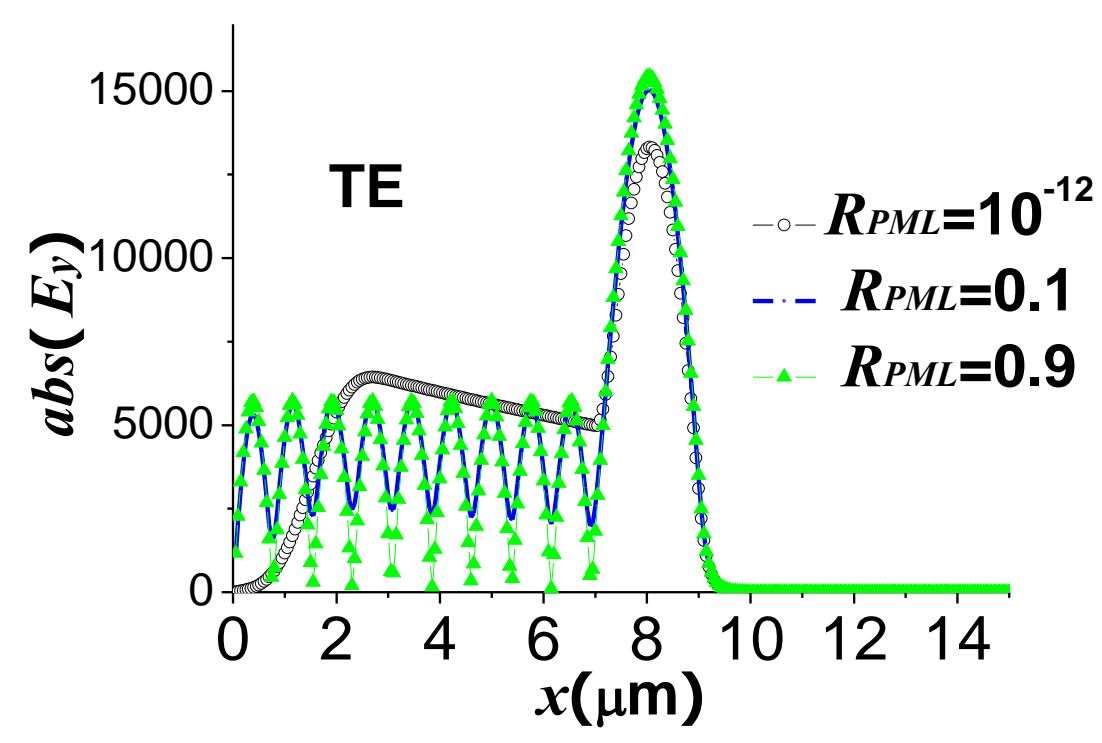

(a)

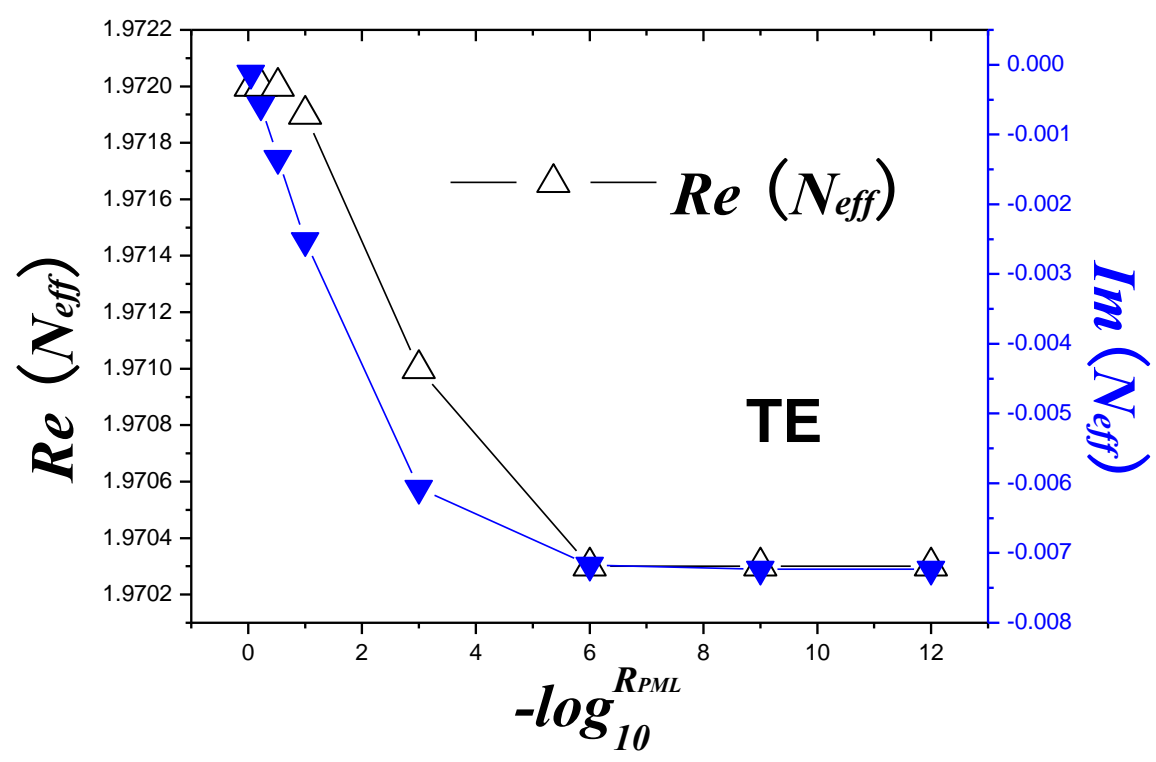

(b)

Figure 5.3. (a) Modal field amplitude patterns for TE polarization (b) Convergence of $N_{\text {eff }}$ with $R_{P M L}$ 


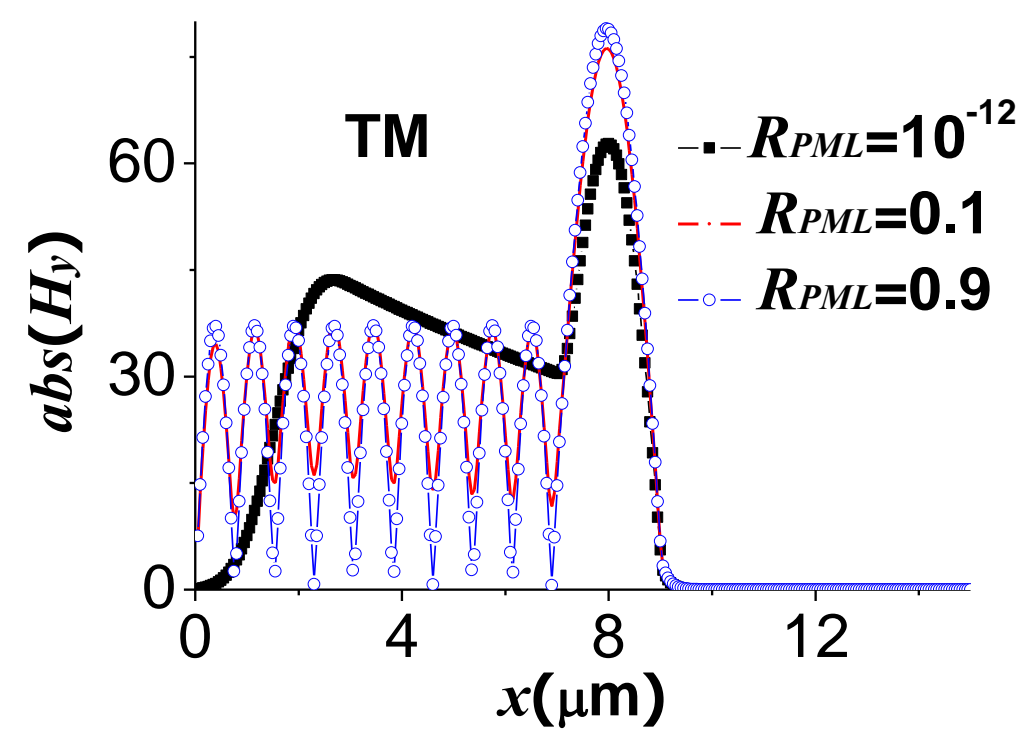

(a)

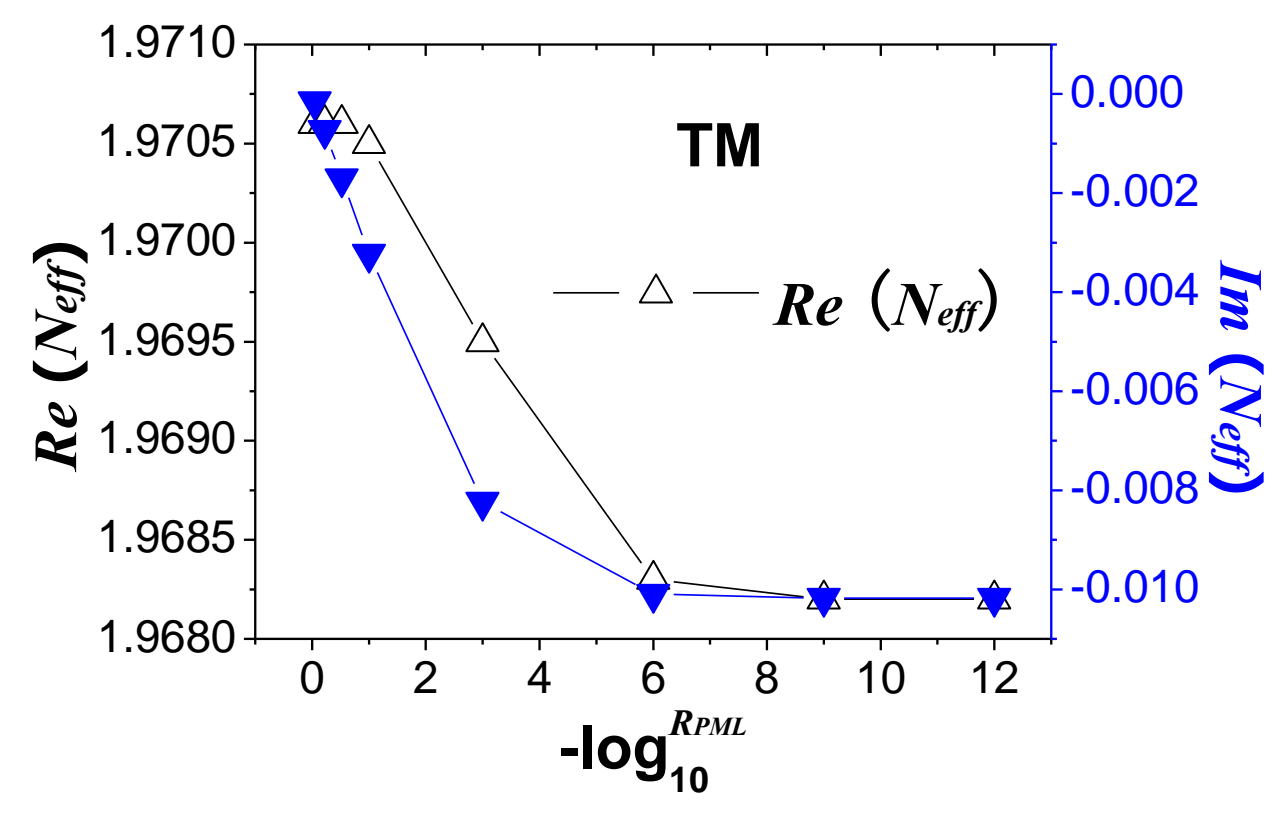

(b)

Figure 5.4. (a) Modal field amplitude patterns in TM polarization (b) Convergence of $N_{\text {eff }}$ with $R_{P M L}$

We normalize $P_{z, \text { in }}$ to be 1 , and set the longitudinal length of the rectangular box as $50 \mu \mathrm{m}$. From Figure 5.5, we can see that the powers flowing into and that out 
of the box are always balanced. With the decreasing of $R_{P M L}, P_{z}$ decaying rate will increase due to the stronger absorption of PML, but eventually converges.

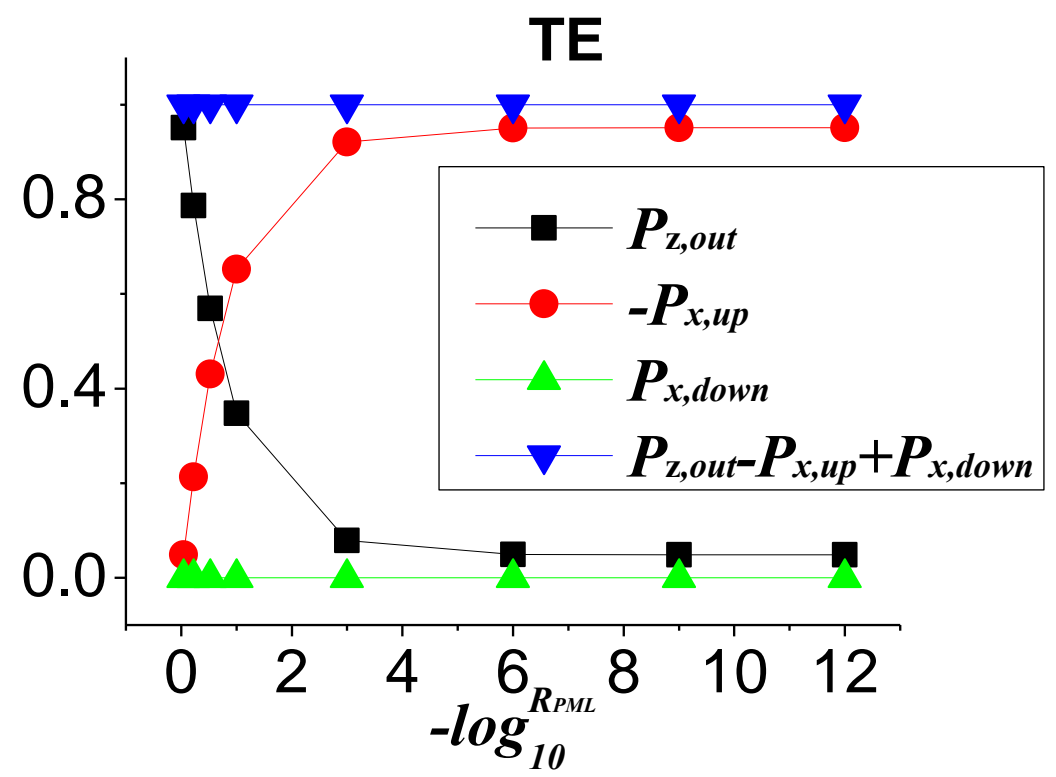

(a)

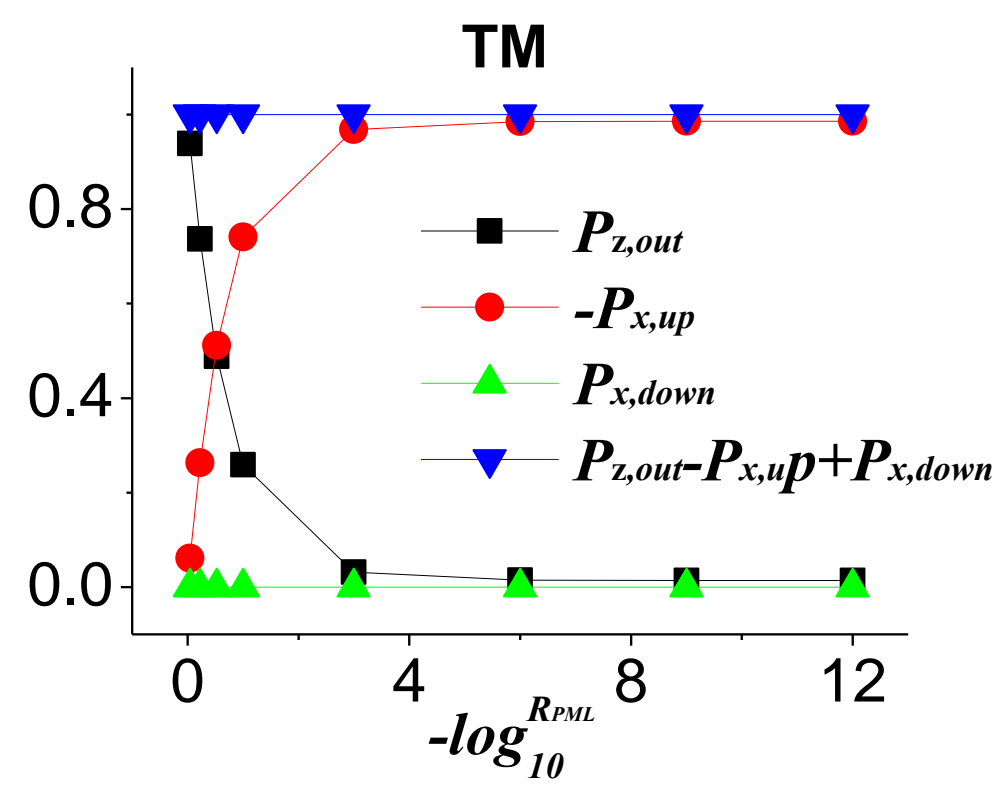

(b)

Figure 5.5. Power conservation of CMMM in leaky waveguide (a) TE (b) TM 


\subsection{Waveguide Discontinuity}

Figure 5.6 shows a waveguide junction between a 2-D slab waveguide and leaky waveguide. A rectangular box is placed in the waveguide region. The fundamental mode is launched at the left side of the box. The longitudinal length of this box is L1+L2. 40 modes are considered. The wavelength is $1.5 \mu \mathrm{m}$.

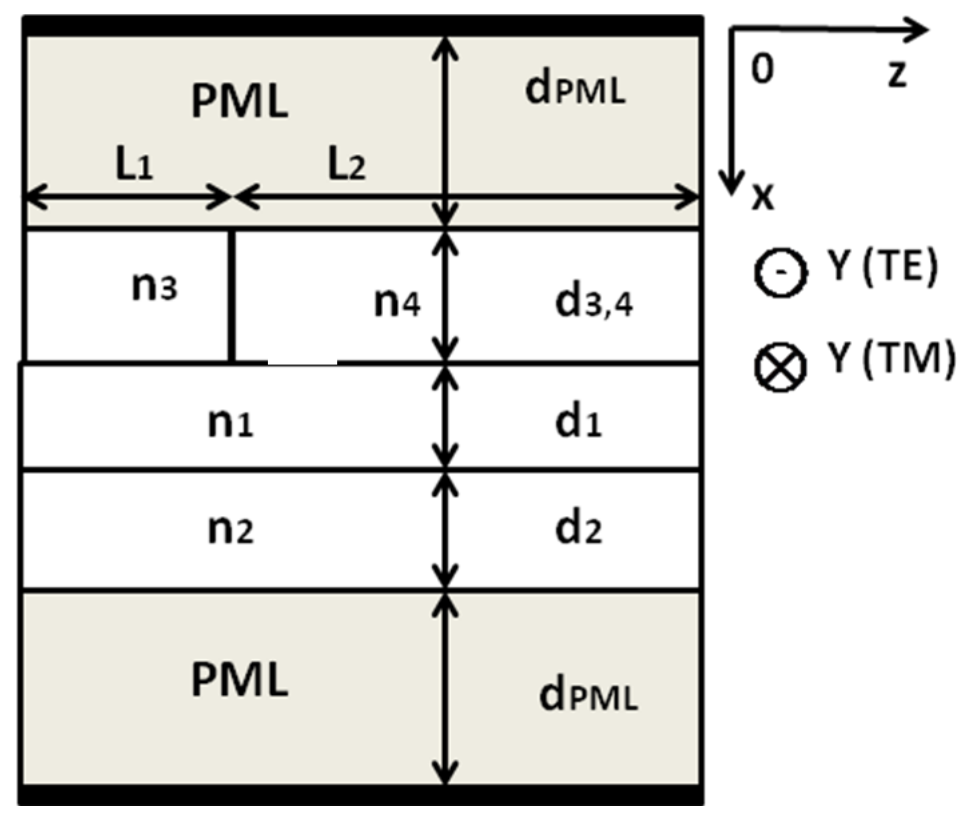

Figure 5.6. The schematic of a junction of $2-\mathrm{D}$ waveguides. $\mathrm{dPML}=3 \mu \mathrm{m}, \mathrm{n} 1=3.4$, $\mathrm{n} 3=3.3, \mathrm{n} 2=1, \mathrm{n} 4=3.5, \mathrm{~d} 1=1 \mu \mathrm{m}, \mathrm{d} 2=2 \mu \mathrm{m}, \mathrm{d} 3,4=2 \mu \mathrm{m}$. L1 $=2 \mu \mathrm{m}, \mathrm{L} 2=50 \mu \mathrm{m}$

After normalizing $P_{z, \text { in }}$ to be 1, Figure 5.7 and 5.8 show the convergence of $P_{z, \text { out }}$ with the number of modes applied and the computation window for TE and TM polarizations, respectively. Figure 5.9 shows the power conservation of the passive box which is independent of $R_{P M L}$. However, smaller $R_{P M L}$ will make $P_{z}$ decay faster, and the convergence happens when $R_{P M L}$ decreases to $1 \mathrm{e}-3$. 


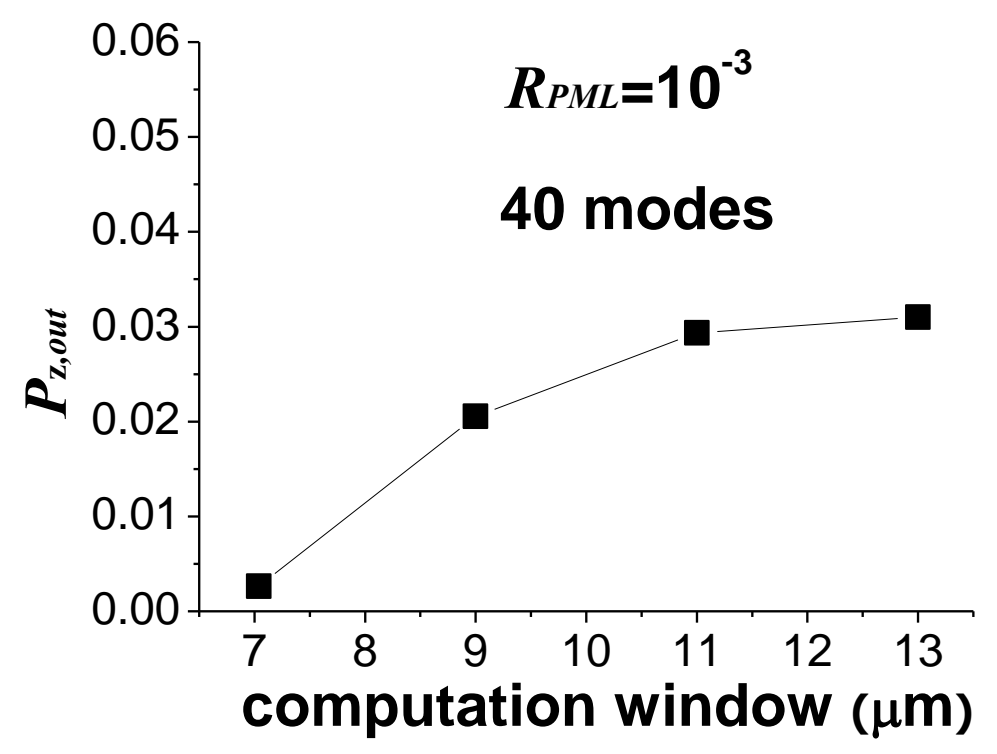

(a)

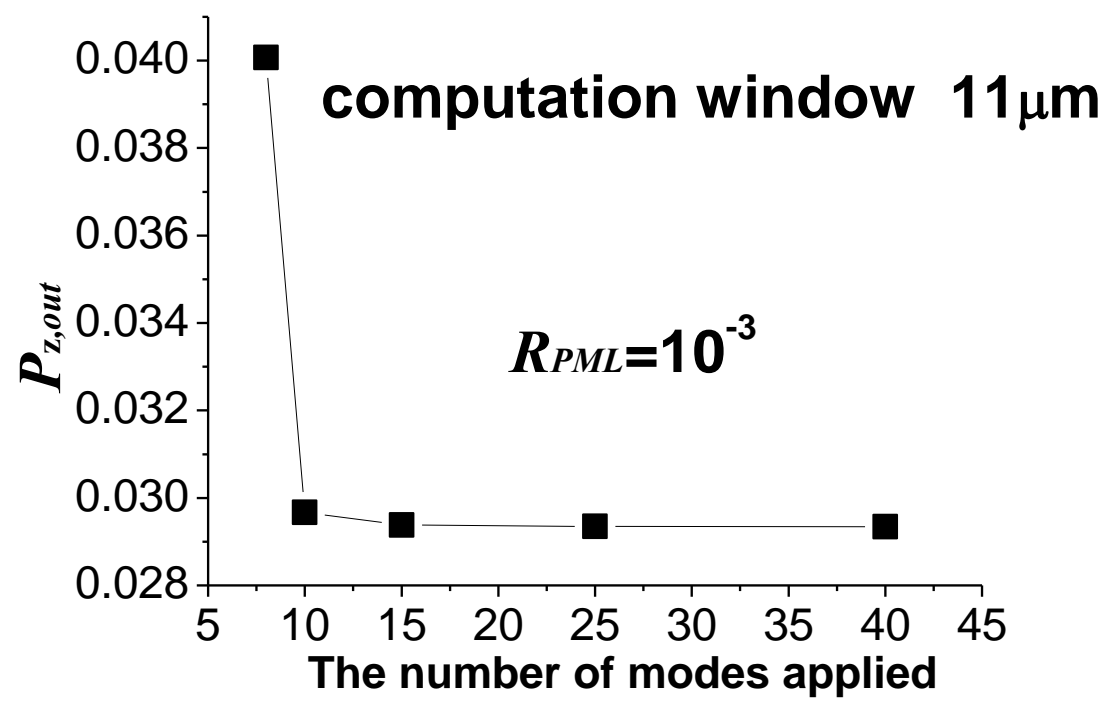

(b)

Figure 5.7. The convergence test of $P_{z, \text { out }}$ (a) about the computation window and (b) the number of modes applied for TE polarization in the waveguide junction (Figure 5.6) 


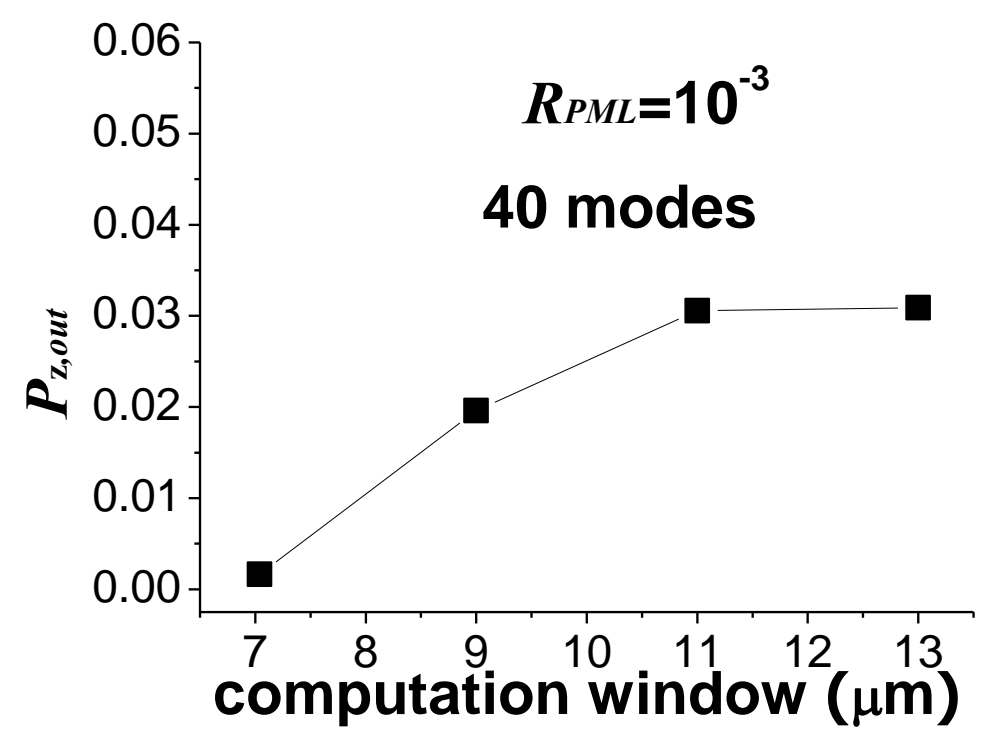

(a)

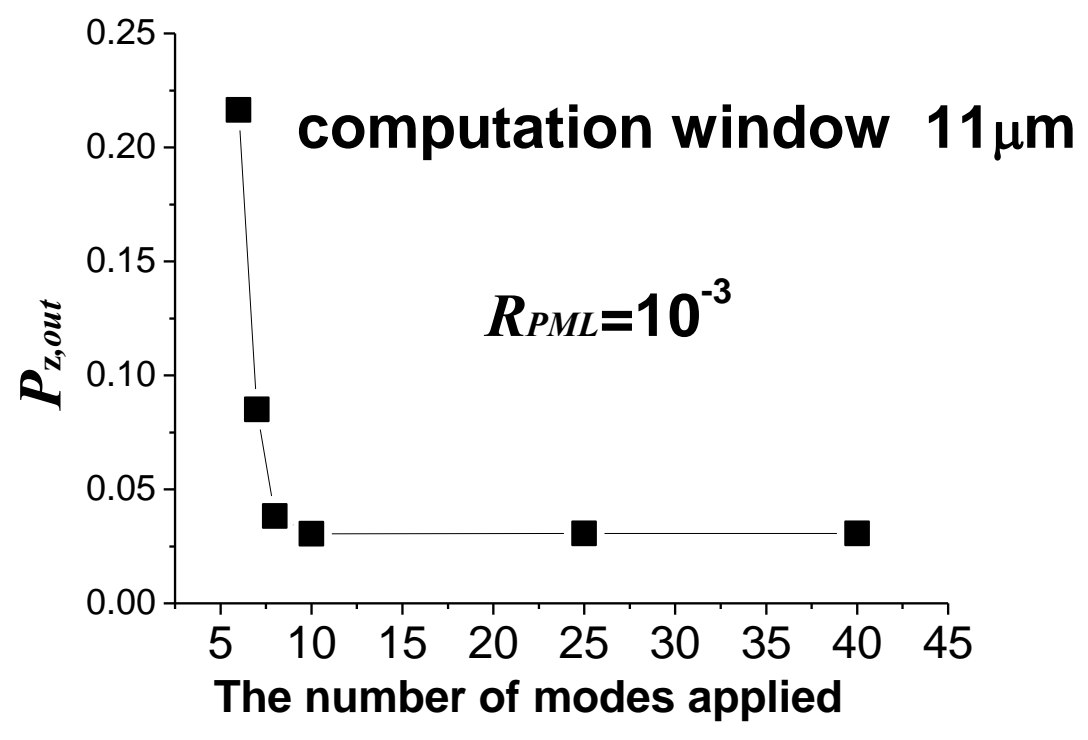

(b)

Figure 5.8. The convergence test of $P_{z, \text { out }}$ (a) about the computation window and (b) the number of modes applied for TM polarization in the waveguide junction (Figure 5.6) 


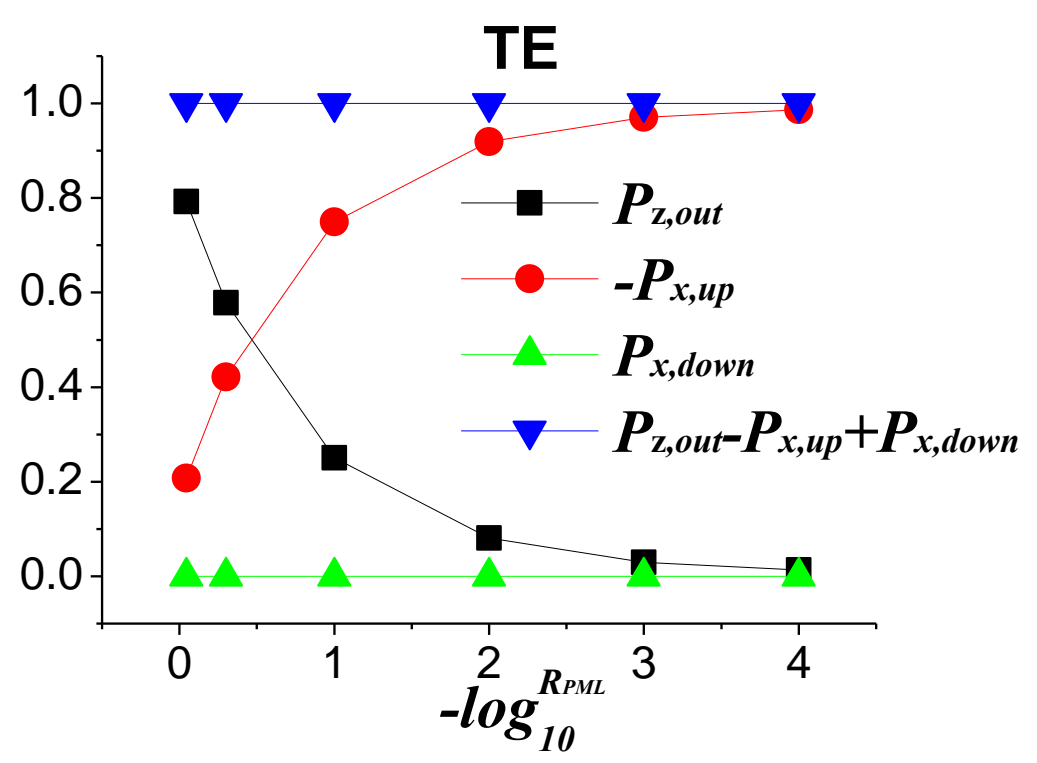

(a)

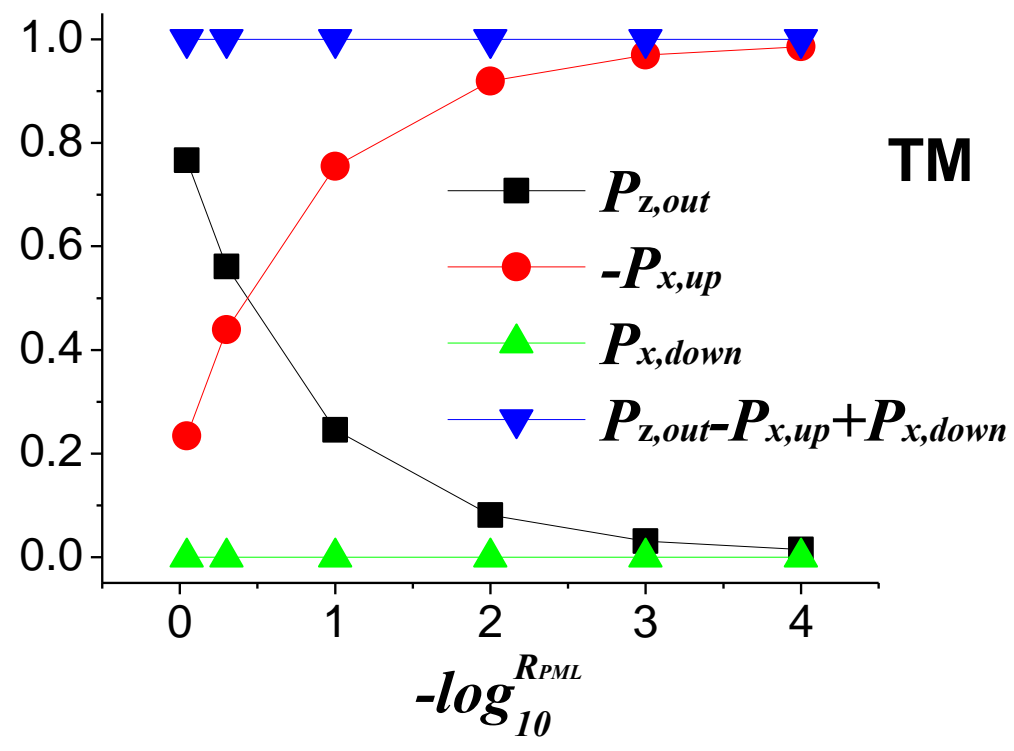

(b)

Figure 5.9. Power conservation of CMMM in the waveguide junction (Figure 5.6) (a) TE (b) TM

Figure 5.10 describes the transversely outgoing electric (magnetic) field amplitude patterns at the right side of the rectangular box in TE (TM) case, 
respectively. We can see that decreased $R_{P M L}$ will promise larger field confinement in PML region, and smaller transverse field amplitude at the right side.

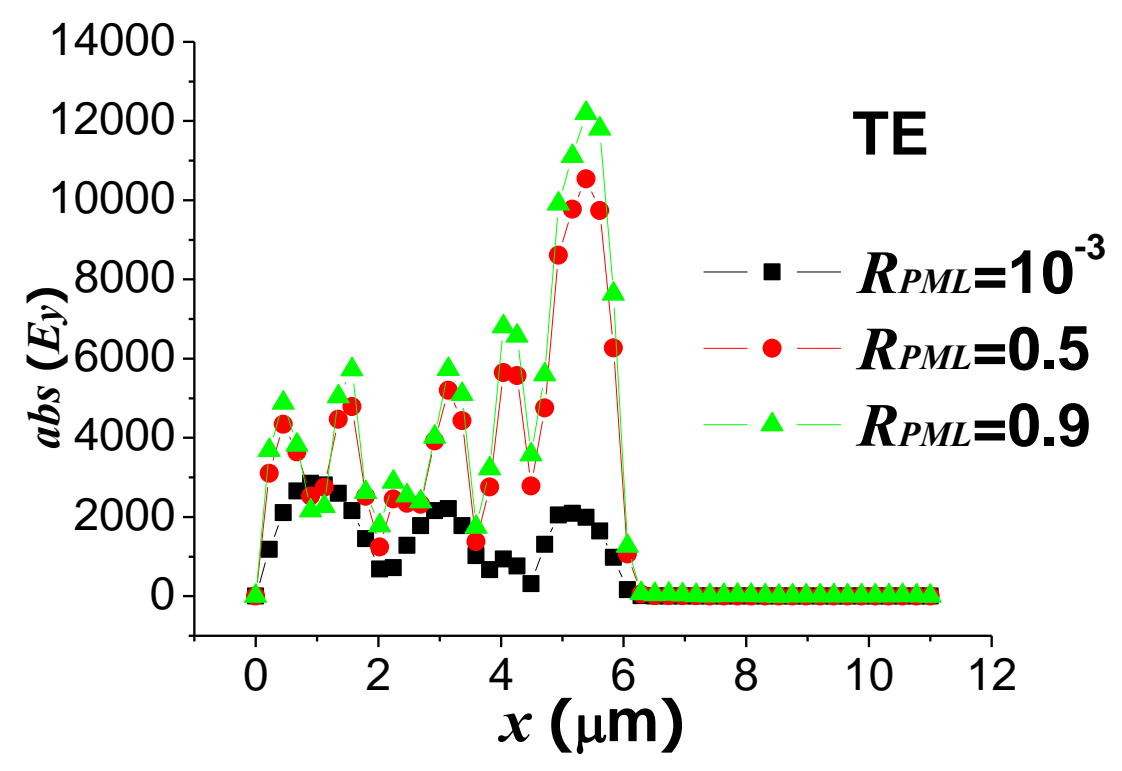

(a)

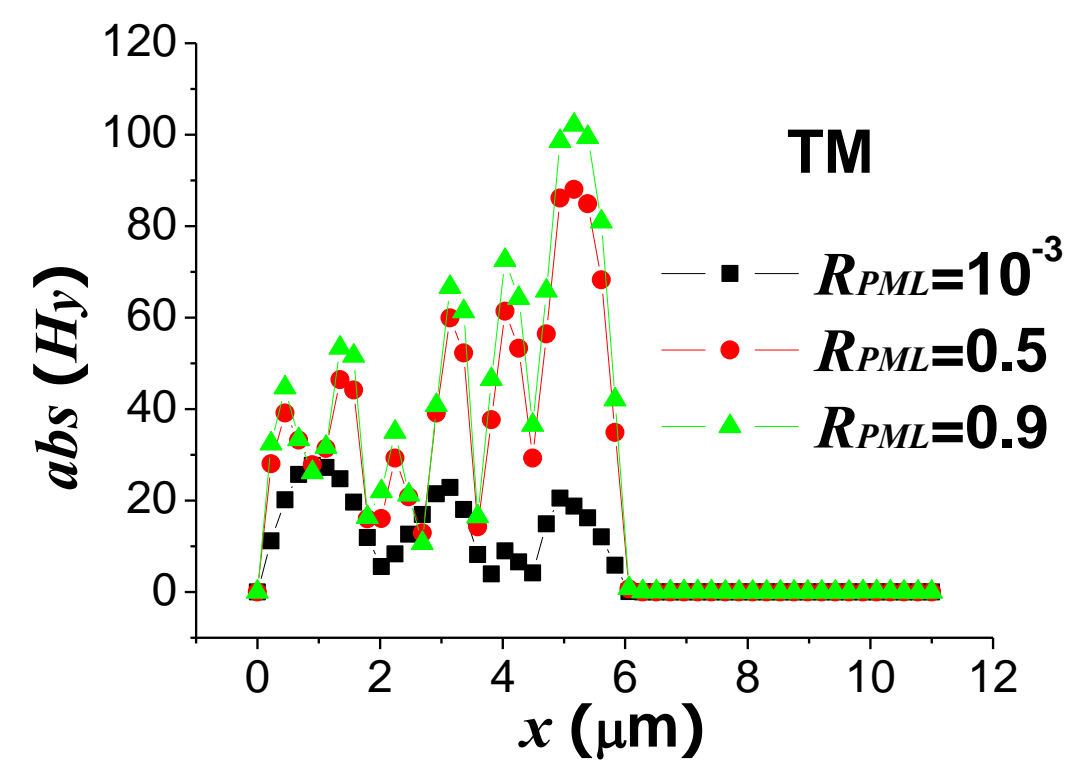

(b)

Figure 5.10. Transversely outgoing field amplitude patterns in the waveguide junction (Figure 5.6) (a) TE (b) TM 
Figure 5.11 shows the vertically outgoing electric (magnetic) field amplitude pattern for TE (TM) polarization which holds larger oscillation for large $R_{P M L}$ due to the interferences of reflected wave from PML.

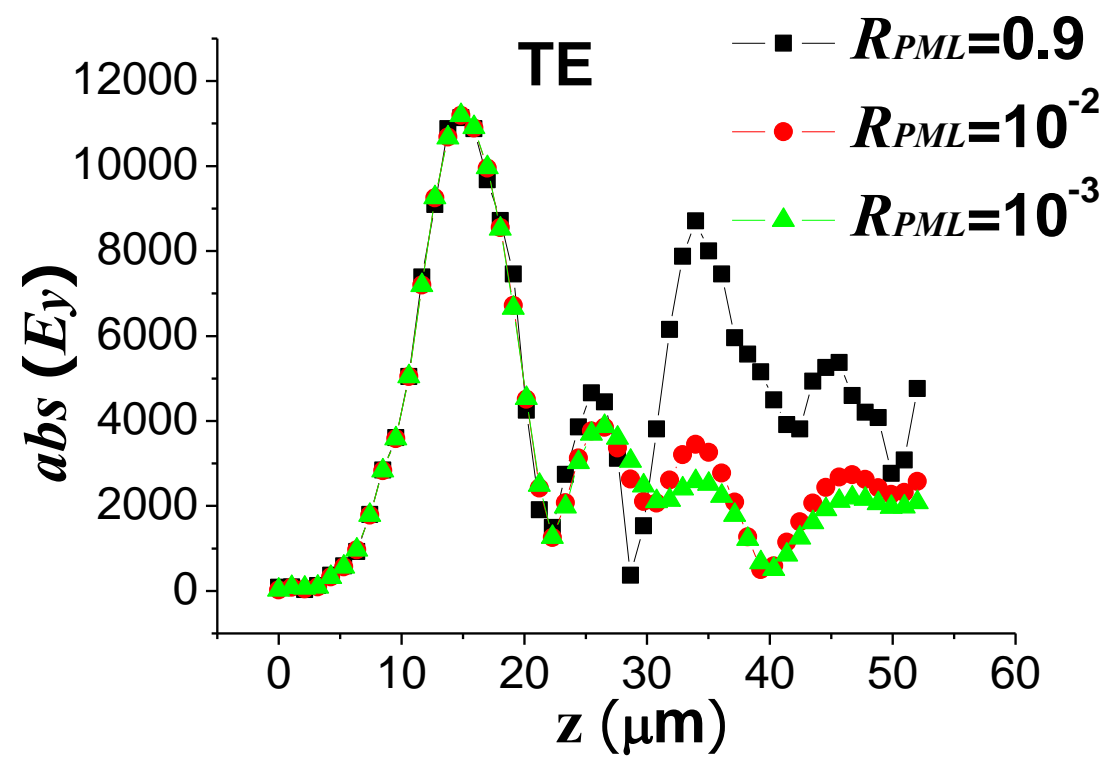

(a)

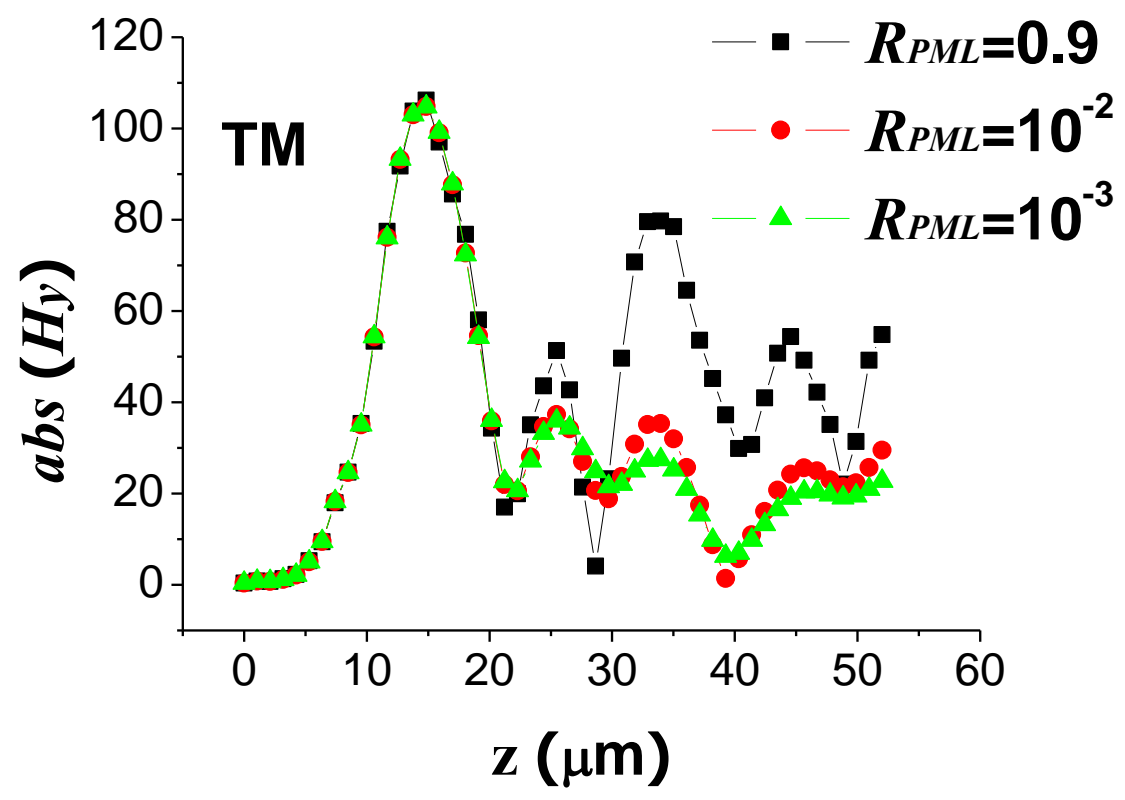

(b)

Figure 5.11. Vertically outgoing field amplitude pattern in the waveguide junction (Figure 5.6) (a) TE (b) TM 


\section{Chapter 6 \\ Simulation of Waveguide Crossings and Corners}

\subsection{Introduction}

High-index-contrast waveguide crossings and corners are highly desired in the industrial applications because of their strong ability of controlling of light wave propagation with the dimensions of optical devices scaled down from those of low-index-contrast devices, now that the light confinement based on total internal reflection strongly depends on the index contrast between waveguide cores and claddings [9-10]. With the knowledge of the power conservation of CMMM, the vertical power flow is easily acquired. In this chapter we for the first time simulate high-index-contrast waveguide crossings and corners and calculate the radiation power perpendicular to the waveguide axis with CMMM and validate the results with FDTD. Figure 6.1 shows the schematic of 2-D waveguide crossings, T-junction and corners. And the transmission, reflection and crosstalk are defined in [11]. 

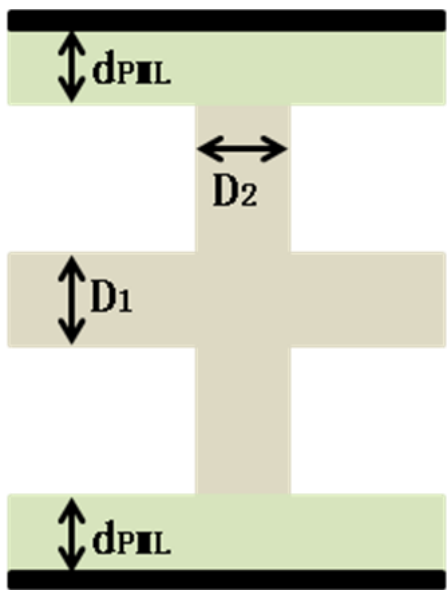

(a)
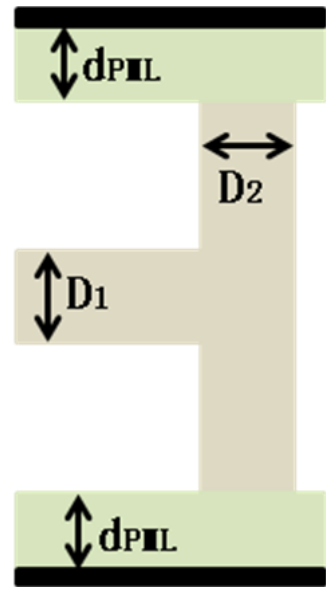

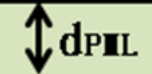

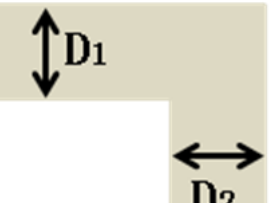

†dPIL

D2
(b)
(a)

Figure 6.1. The schematics of 2-D (a)waveguide crossing (b)waveguide T-junction (c)waveguide corner terminated with PML and PRB.

\subsection{Simulation of High-Index-Contrast Waveguide Crossings and Corners}

\subsection{Waveguide Crossing}

We follow the case of waveguide crossing in [11], and in Figure 6.1 we have $\mathrm{D} 1=\mathrm{D} 2=0.2 \mu \mathrm{m}, \mathrm{dPML}=5 \mu \mathrm{m}$, the fiber and background indices are 3.2 and 1 , respectively. At the wavelength of $1.48 \mu \mathrm{m}$, we do the convergence test for this high index-contrast waveguide crossing in Figure 6.2. 


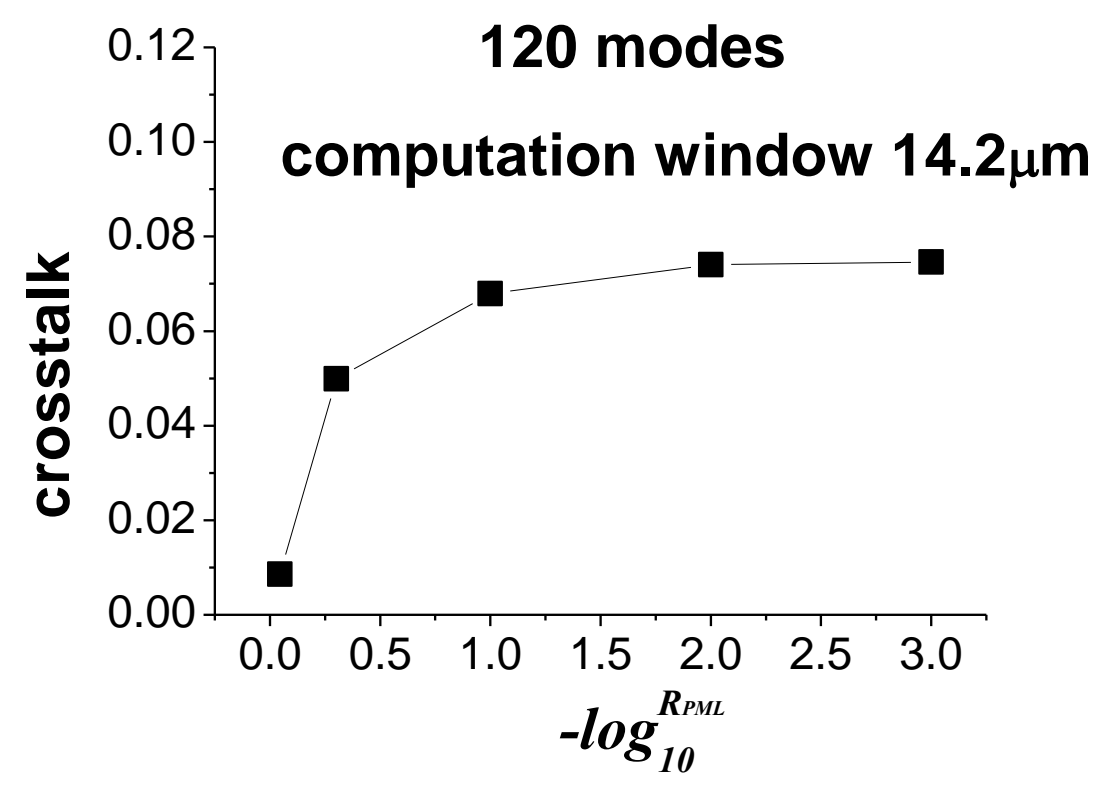

(a)

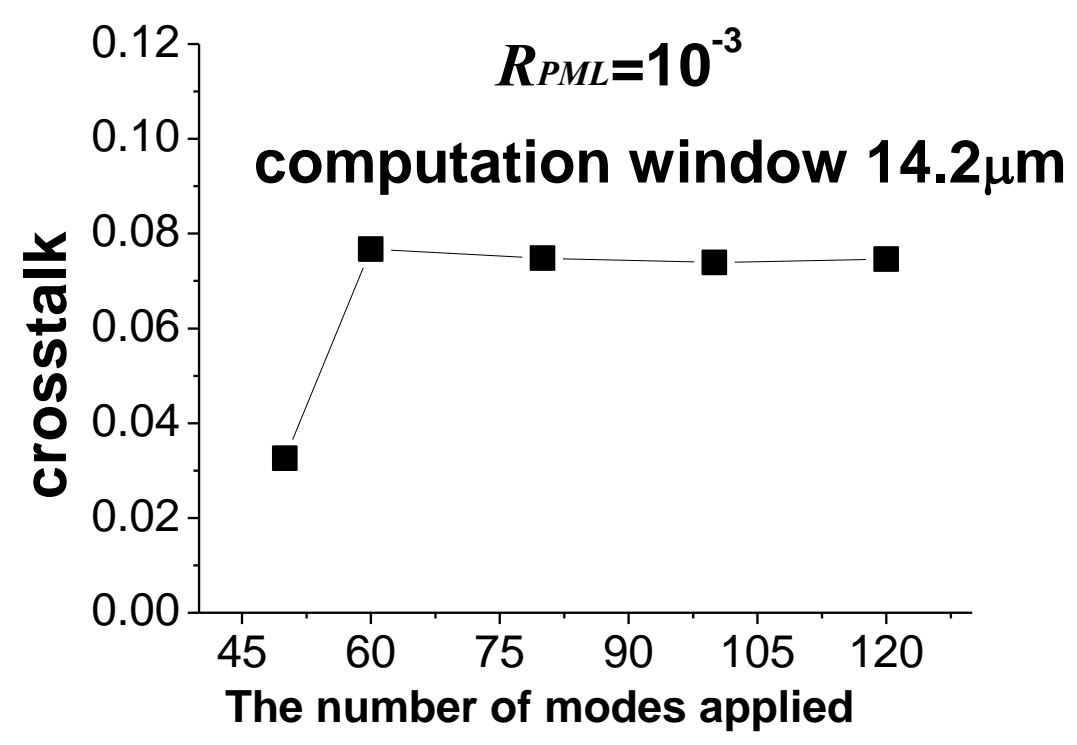

(b) 


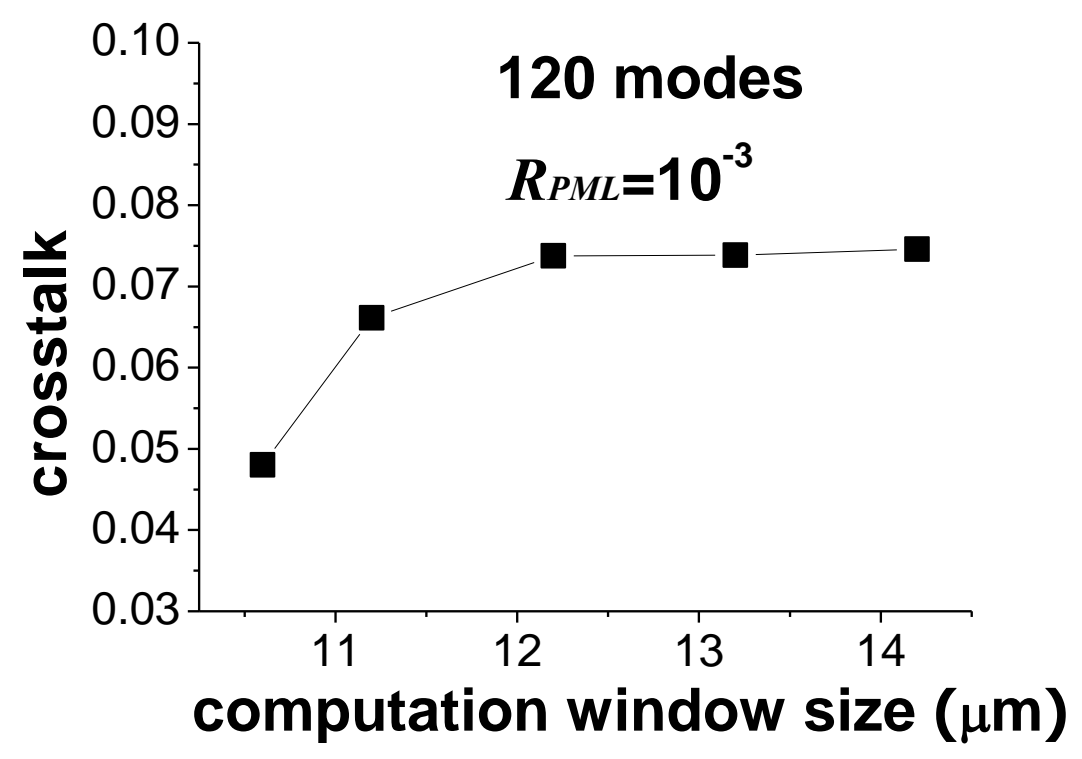

(c)

Figure 6.2. Convergence test of guided crosstalk about (a) $R_{P M L}$ (b) the number of modes applied, and (c) the computation window in the high-index-contrast waveguide crossing

With large enough computation window and adequate complex modes applied, the combination of complex modes will represent the continuous radiation field well enough, thus the guided crosstalk converges. The smaller $R_{P M L}$ makes the absorption of vertically radiating power in PML stronger, thus the guided crosstalk will increase and eventually converges.

With the computation window as $14.2 \mu \mathrm{m}, 120$ modes considered and $R_{P M L}$ as 1e-3, we investigate guided transmission and crosstalk spectra in Figure 6.3 and validate it with that of FDTD in [11]. 


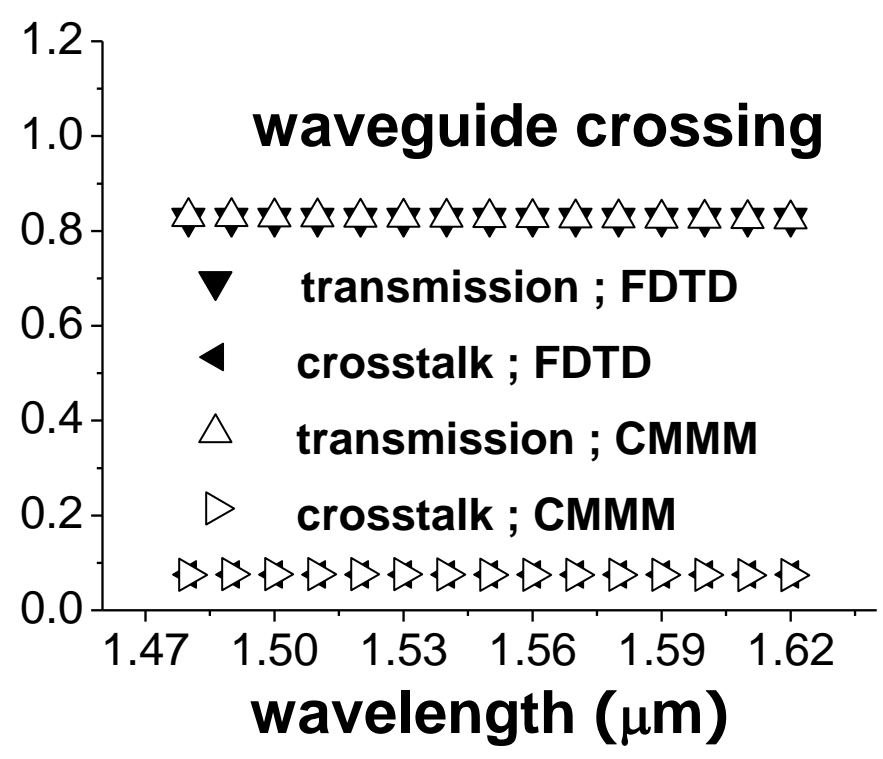

Figure 6.3. Guided transmission and crosstalk spectra in the high-index-contrast waveguide crossing

Figure 6.4 and 6.5 study the effect of the variation of the background index (D2=0.2 $\mu \mathrm{m}$ ) and D2 (the background index is 1) in high-index-contrast waveguide crossing.

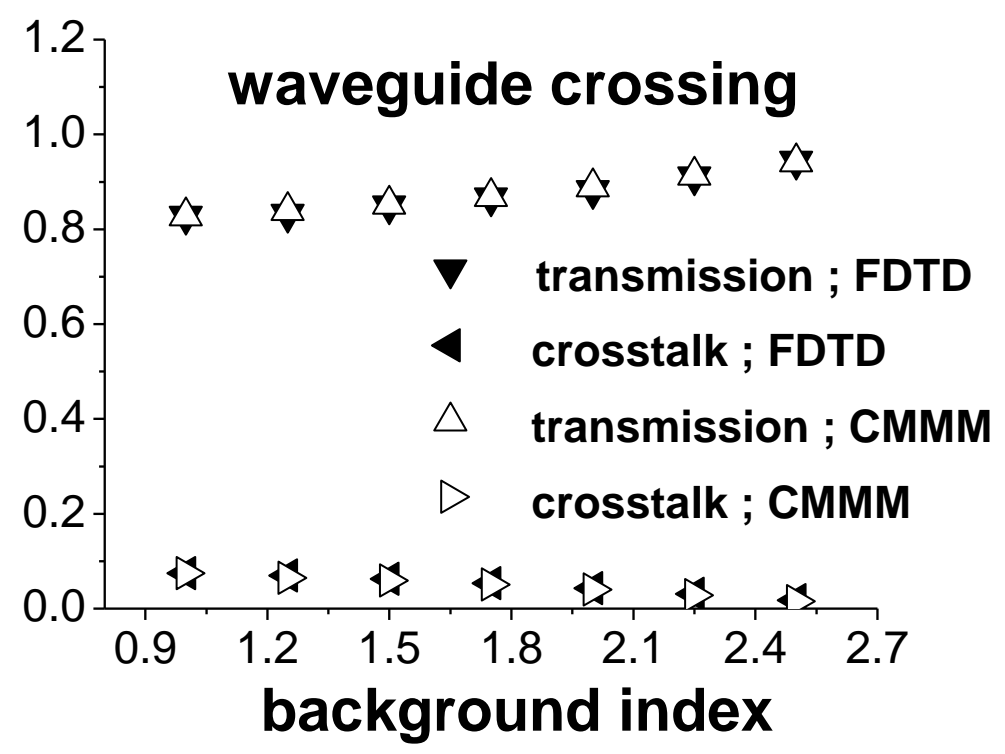

Figure 6.4. The evolutions of guided transmission and crosstalk with the variation of background index in high-index-contrast waveguide crossing 


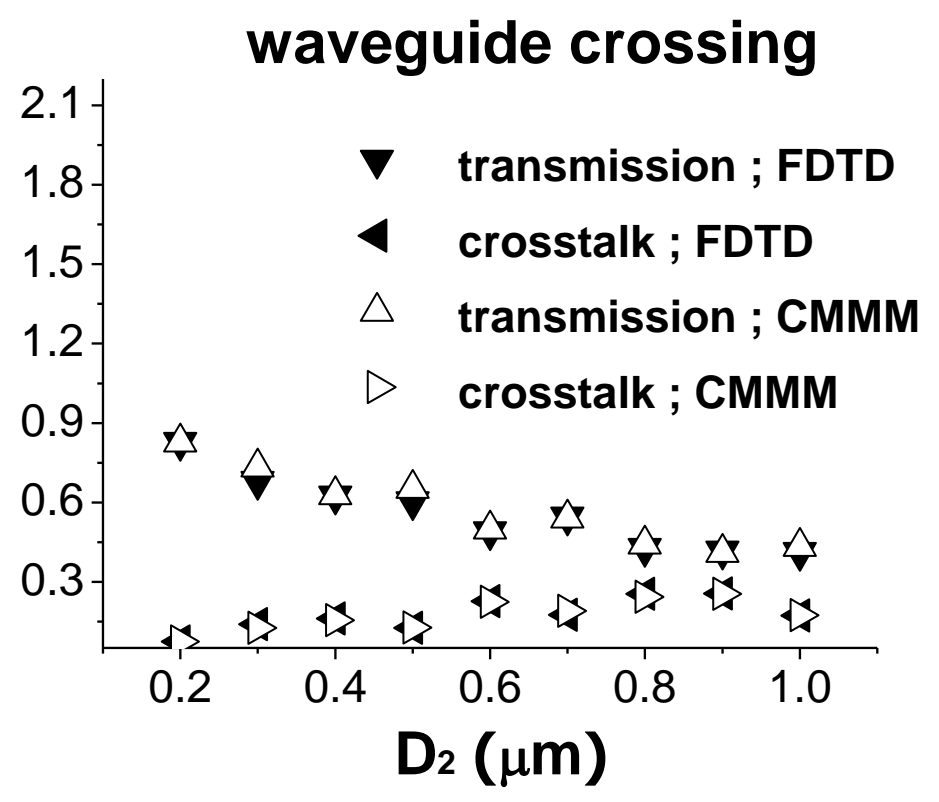

Figure 6.5. The evolutions of guided transmission and crosstalk with D2 in highindex-contrast waveguide crossing.

At the wavelength of $1.55 \mu \mathrm{m}, \mathrm{D} 2$ of $0.2 \mu \mathrm{m}$, and the background index of 1, Figure 6.6 demonstrates the electric field amplitude pattern in this high index-contrast waveguide crossing, which coincide well with that of [11].

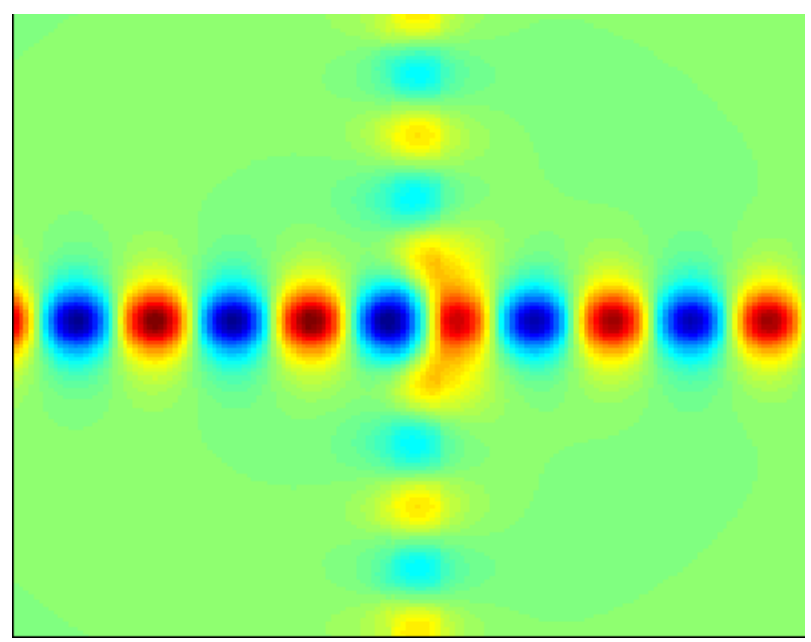

Figure 6.6. Electric field amplitude pattern in the high-index-contrast waveguide crossing 


\subsection{Waveguide T-junction}

Following the examples of [11], in Figure 6.1 we have D1=D2=0.2 $\mu \mathrm{m}, \mathrm{dPML}=5 \mu \mathrm{m}$. At the fiber and background indices of 3.2 and 1, respectively, and the wavelength of $1.48 \mu \mathrm{m}$, Figure 6.7 shows the convergence test about this T-junction. Further Figure 6.8 demonstrates the guided transmission and reflection spectra.

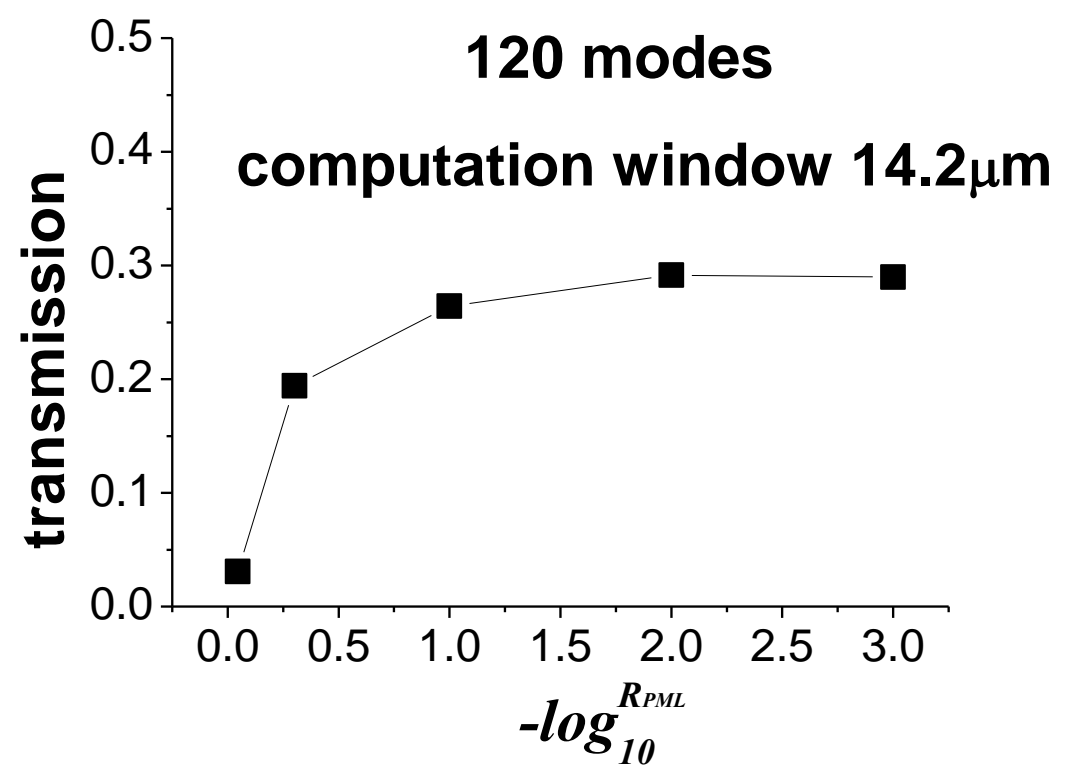

(a) 


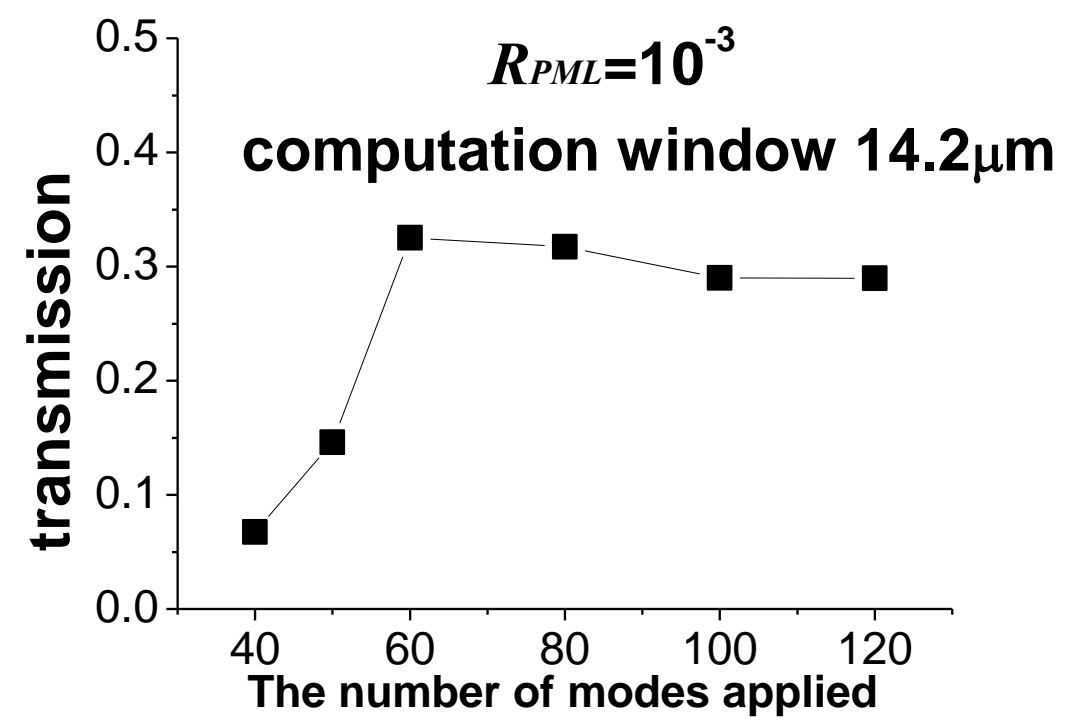

(b)

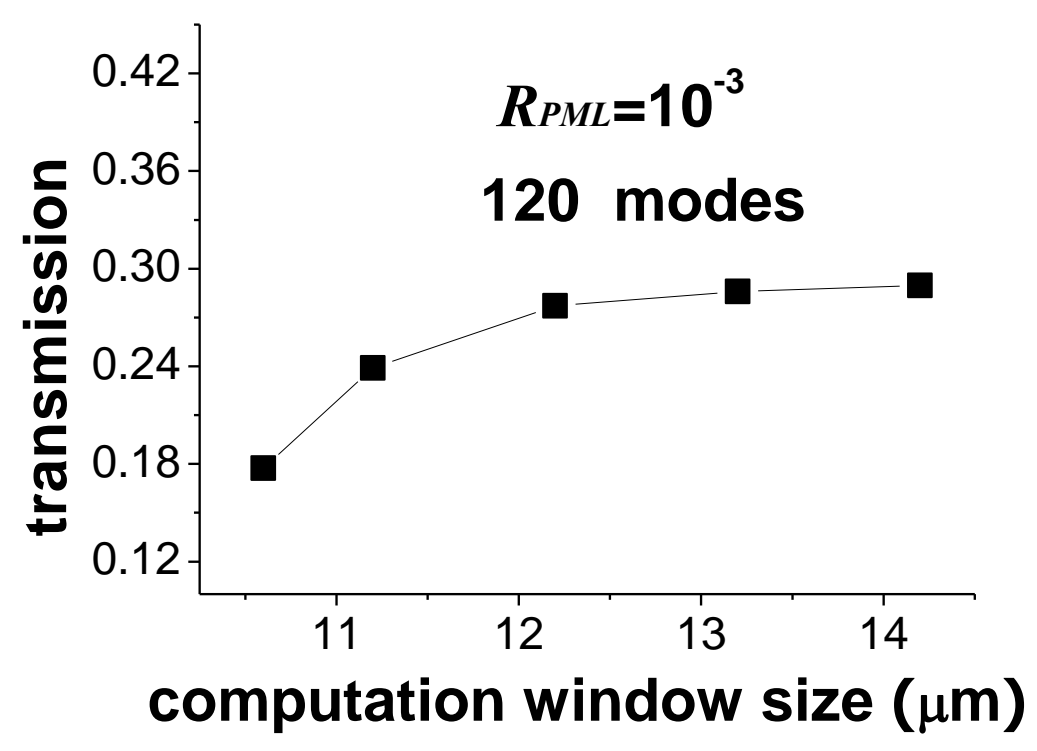

(c)

Figure 6.7. Convergence test of guided transmission about (a) $R_{P M L}$ (b) the number of modes applied and (c) computation window in high-index-contrast waveguide T-junction. 


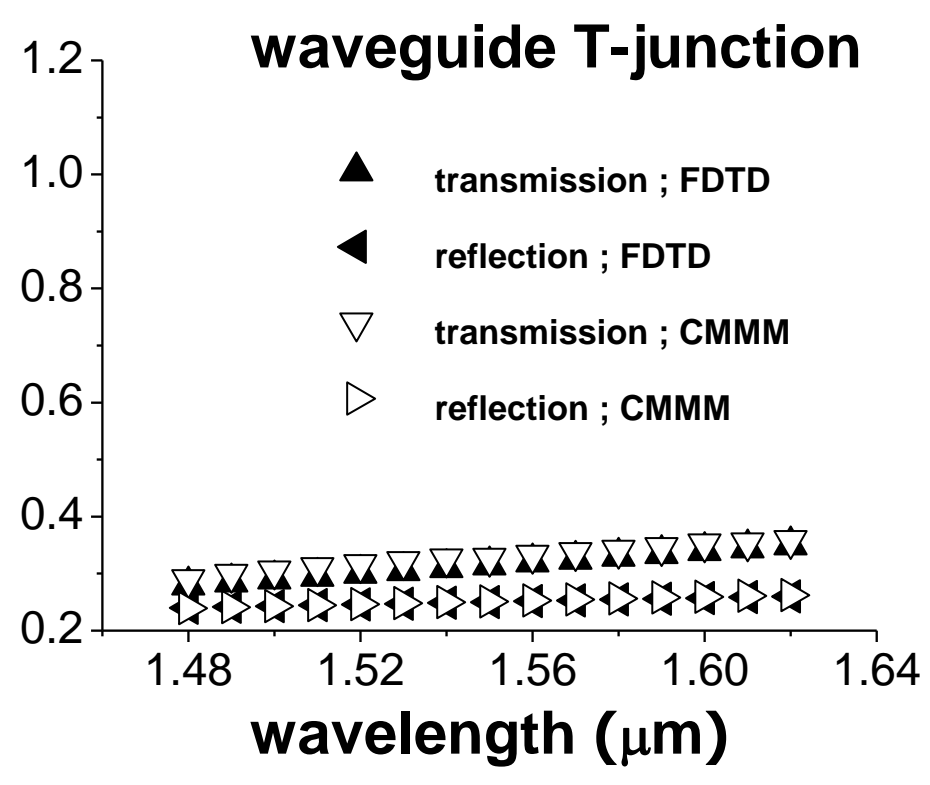

Figure 6.8. Guided transmission and reflection spectra in the high-index-contrast T-junction

Figure 6.9 and 6.10 show the impact of the variation of the background index (D2=0.2 $\mu \mathrm{m})$ and D2 (the background index is 1$)$, respectively.

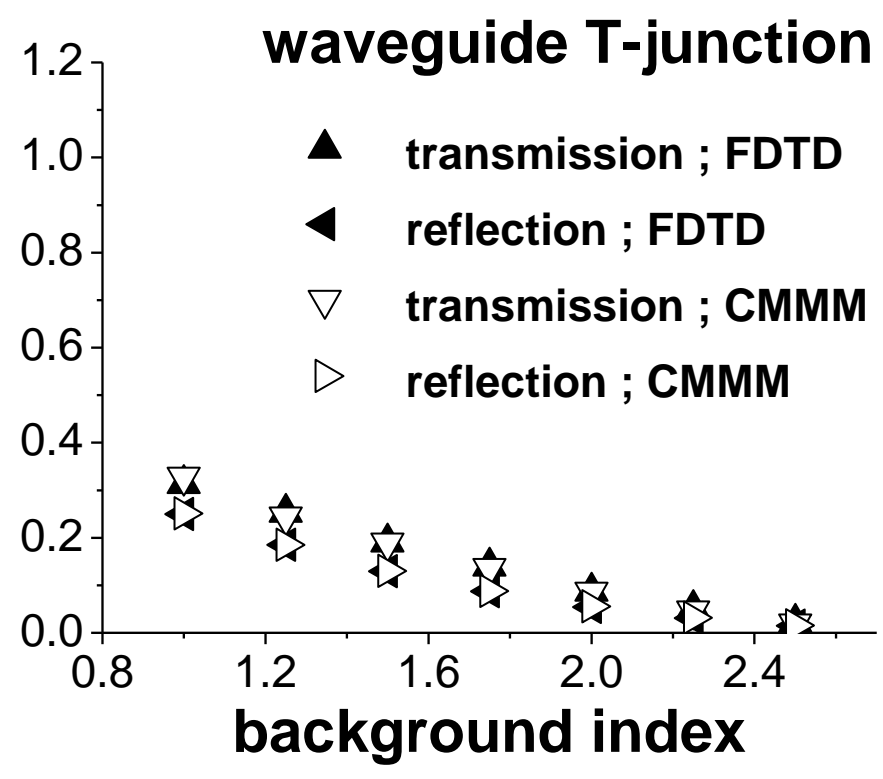

Figure 6.9. The evolution of guided transmission and reflection with the variation of background index in the high-index-contrast waveguide T-junction 


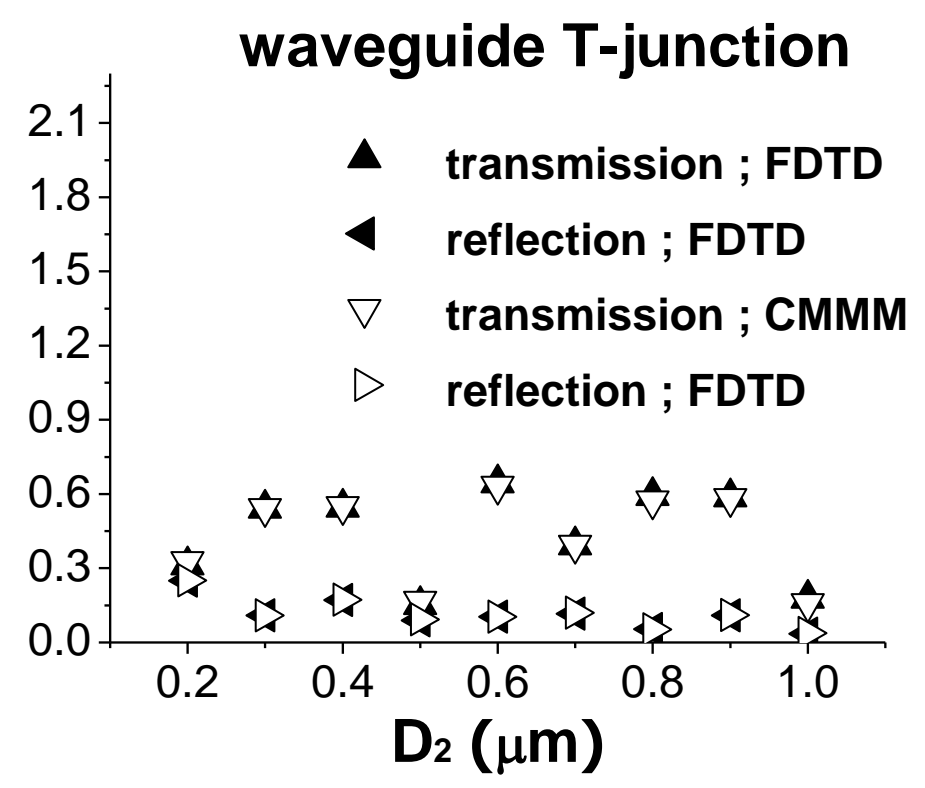

Figure 6.10. The evolution of guided transmission and reflection with the variation of $\mathrm{D} 2$ in the high-index-contrast waveguide T-junction

Figure 6.11 shows the electric field amplitude pattern at the wavelength of $1.55 \mu \mathrm{m}, \mathrm{D} 2$ of $0.2 \mu \mathrm{m}$ and the background index of 1 .

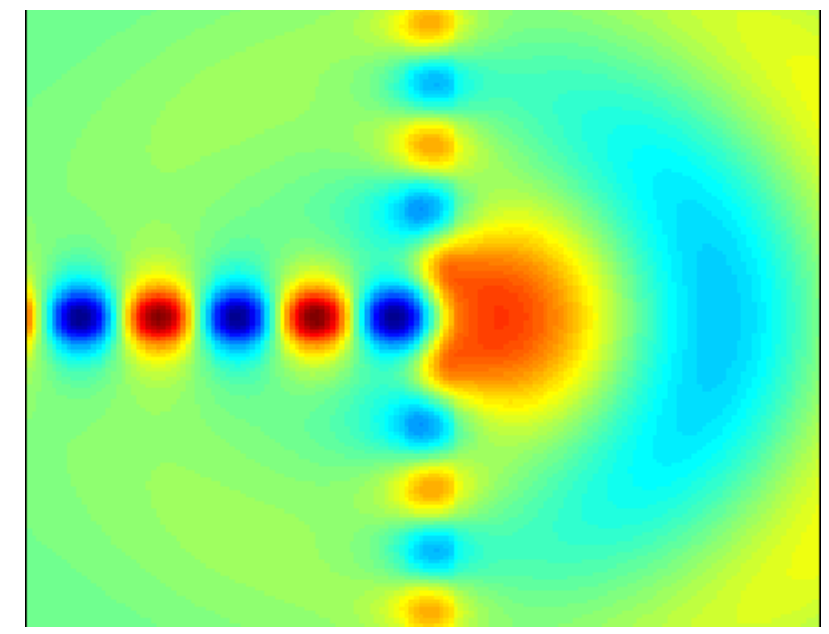

Figure 6.11. Electric field amplitude pattern in the high-index-contrast T-junction

\section{23 Waveguide Corner}

Again we follow the example of [11], and set $\mathrm{D} 2=\mathrm{D} 1=0.2 \mu \mathrm{m}$, and $\mathrm{dPML}=5 \mu \mathrm{m}$ in

Figure 6.1. The fundamental mode is launched from the left horizontal port, with 
electric field polarization perpendicular to the plane. Figure 6.12 shows the convergence test at the wavelength of $1.48 \mu \mathrm{m}$. And Figure 6.13 demonstrates the guided transmission and reflection spectra.

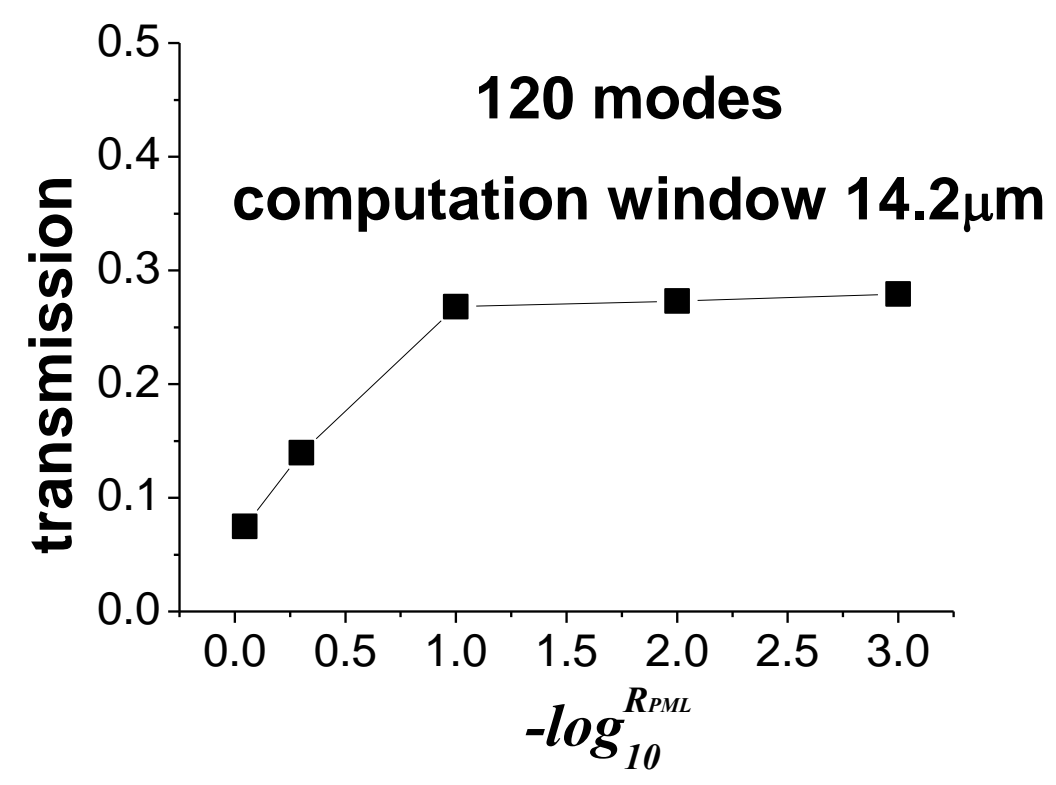

(a)

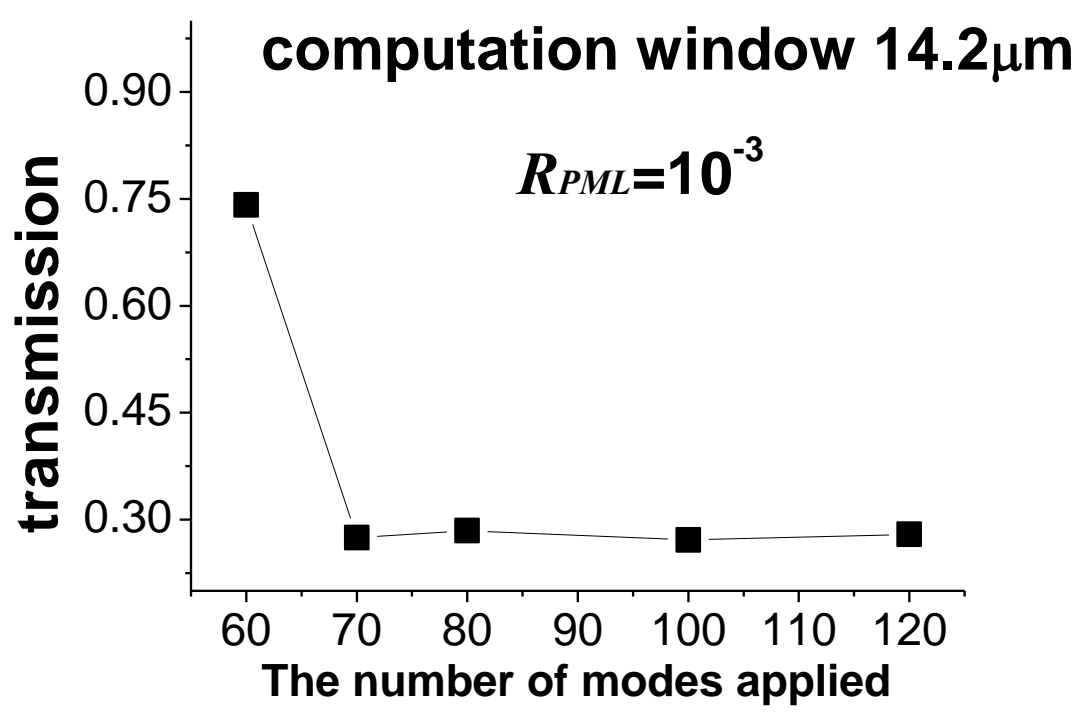

(b) 


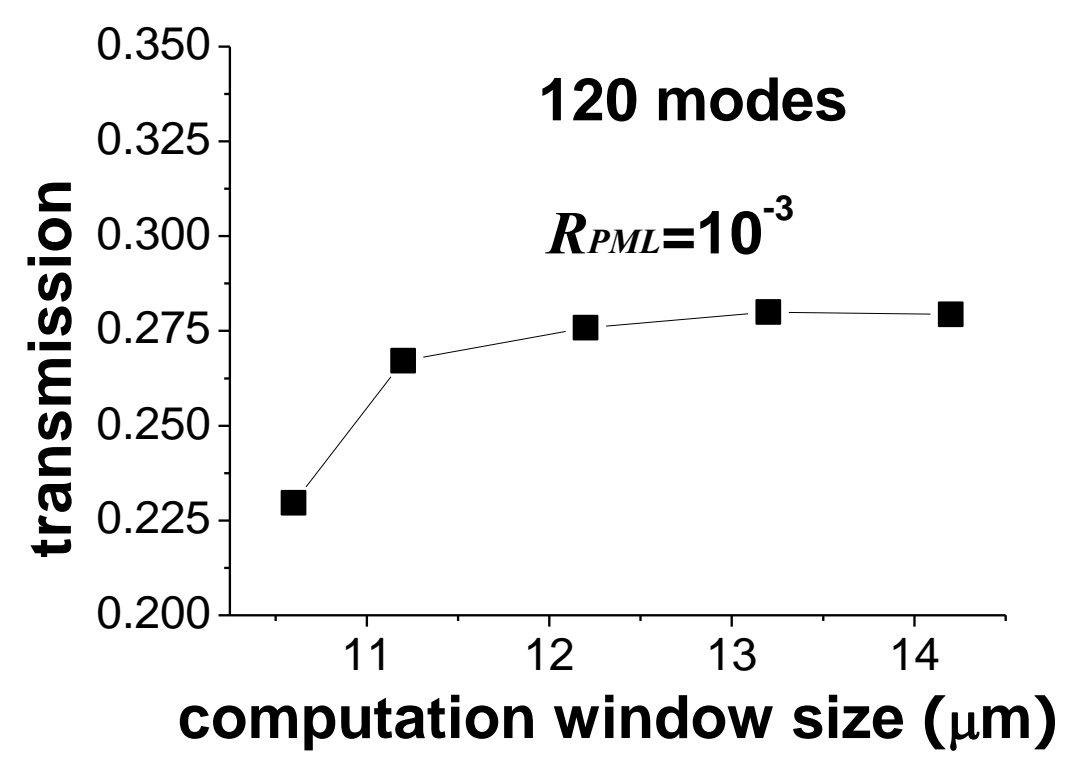

(c)

Figure 6.12. Convergence of guided transmission about (a) $R_{P M L}$ (b) the number of modes applied and (c) the computation window in the high-index-contrast waveguide corner

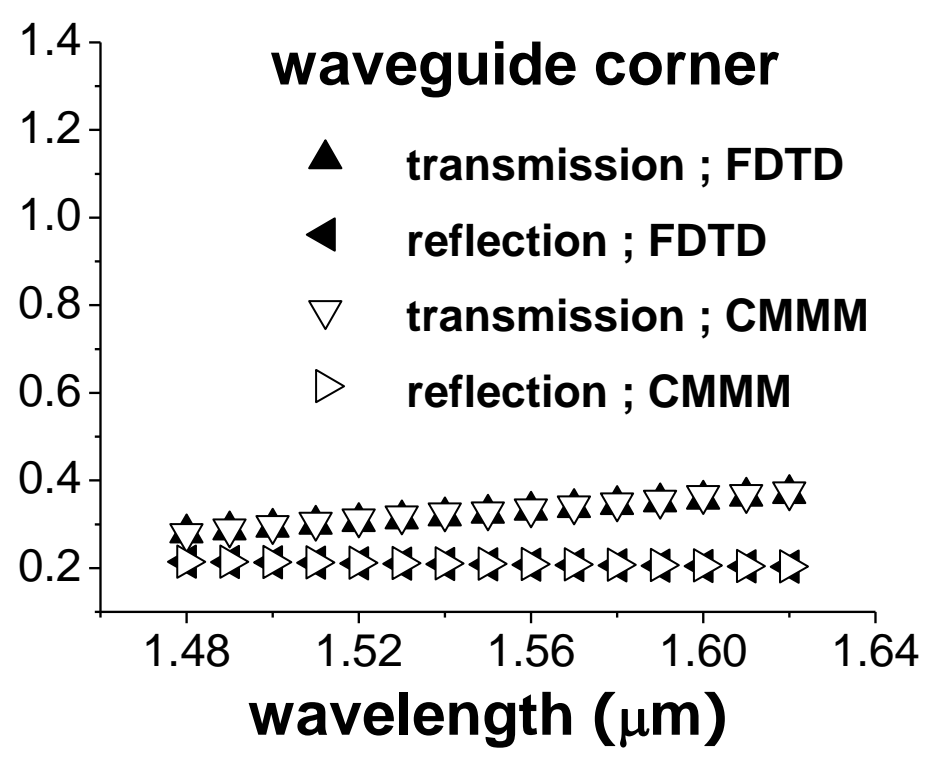

Figure 6.13. Guided transmission and reflection spectra in the high-index-contrast waveguide corner

Figure 6.14 and 6.15 study the influence of the variation of background index (D2=0.2 $\mu \mathrm{m}$ ) and D2 (the background index is 1), respectively. 


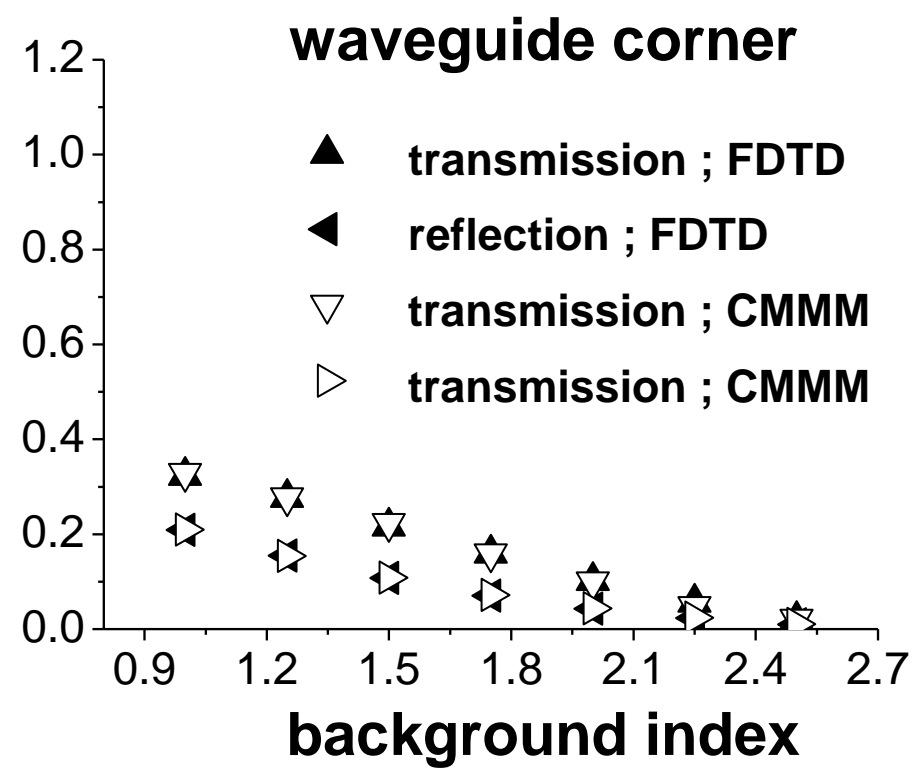

Figure 6.14. The evolutions of guided transmission and reflection with the variation of background index in high-index-contrast waveguide corner

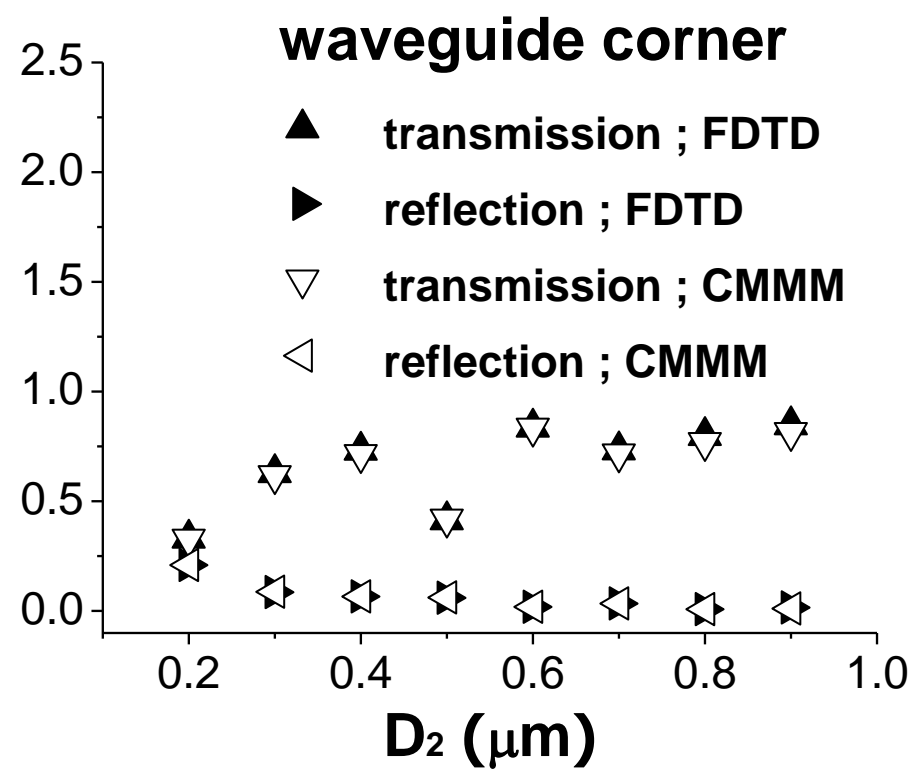

Figure 6.15. The evolutions of guided transmission and reflection with the variation of D2 in high index-contrast waveguide corner

Figure 6.16 shows the electric field amplitude pattern at the wavelength of $1.55 \mu \mathrm{m}$, with D2 as $0.2 \mu \mathrm{m}$ and the background index as 1 . 


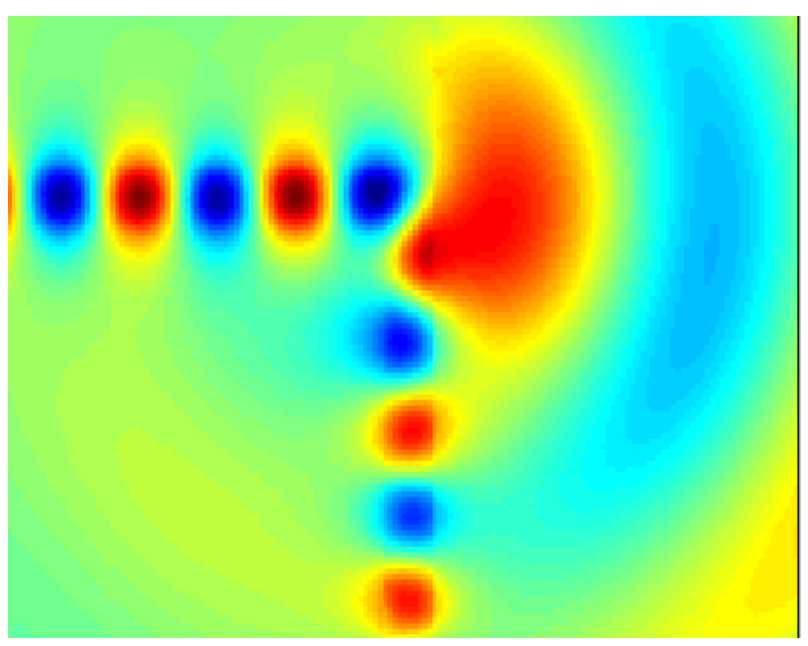

Figure 6.16. Electric field amplitude pattern in high-index-contrast waveguide corner

In summary, the very small relative deviations between sampled spectrums from CMMM and FDTD in high-index-contrast waveguide intersections shown in Table 3 further illustrate the accuracy of CMMM. Additionally Table 4 and Table 5 demonstrate the small relative deviations of sampled transmission, crosstalk or reflection from CMMM and FDTD mentioned above in the high-index-contrast waveguide intersections when the background index and D2 vary, respectively.

Table 3. Relative deviations between the sampled spectrums of CMMM and FDTD in high-index-contrast waveguide intersections

$\begin{array}{cccc} & \text { transmission } & \text { reflection } & \text { crosstalk } \\ \text { waveguide crossing } & 0.000 & & 0.000 \\ \text { waveguide T-junction } & 0.001 & 0.000 & \\ \text { waveguide corner } & 0.003 & 0.000 & \end{array}$

Table 4. Relative deviations of sampled transmission, crosstalk or reflection of CMMM and FDTD in high-index-contrast waveguide intersections with the background index varying ( $\mathrm{D}_{2}$ is $0.2 \mu \mathrm{m}$ )

$\begin{array}{lccc} & \text { transmission } & \text { reflection } & \text { crosstalk } \\ \text { waveguide crossing } & 0.000 & & 0.000 \\ \text { waveguide T-junction } & 0.000 & 0.000 & \\ \text { waveguide corner } & 0.000 & 0.000 & \end{array}$


Table 5. Relative deviations of sampled transmission, crosstalk or reflection of CMMM and FDTD in high-index-contrast waveguide intersections with D2 varying (the background index is 1 )

$\begin{array}{lccc} & \text { transmission } & \text { reflection } & \text { crosstalk } \\ \text { waveguide crossing } & 0.001 & & 0.000 \\ \text { waveguide -junction } & 0.000 & 0.000 & \\ \text { waveguide corner } & 0.001 & 0.000 & \end{array}$

\subsection{T-junction with Cavity}

We consider a T-junction and another one assisted with a square cavity in Figure 6.17.

The fiber index is 3.2 and the background index is 1 . The fundamental mode is launched from the left horizontal port, with electric field perpendicular to the plane. The definitions of the power flow of transmission (PT), crosstalk (PC) and reflection (PR) are shown in Figure 6.17. The mesh size is $20 \mathrm{~nm}$. At the wavelength of $1.55 \mu \mathrm{m}$, Figure 6.18 and 6.19 show the convergence tests of the T-junction and the cavity-assisted one. With $R_{P M L}=1 \mathrm{e}-3,110$ (100) modes considered and computation window as $16.82 \mu \mathrm{m}(16.22 \mu \mathrm{m})$ for the cavity-assisted T-junction (T-junction), we studied the guided transmission, crosstalk and reflection spectra in Figure 6.20. We can see that the resonant cavity increases the crosstalk. Figure 6.21 describes the electric field amplitude patterns in cavity-assisted T-junction obtained from CMMM and FDTD, respectively. The coincidences between CMMM and FDTD are good. 


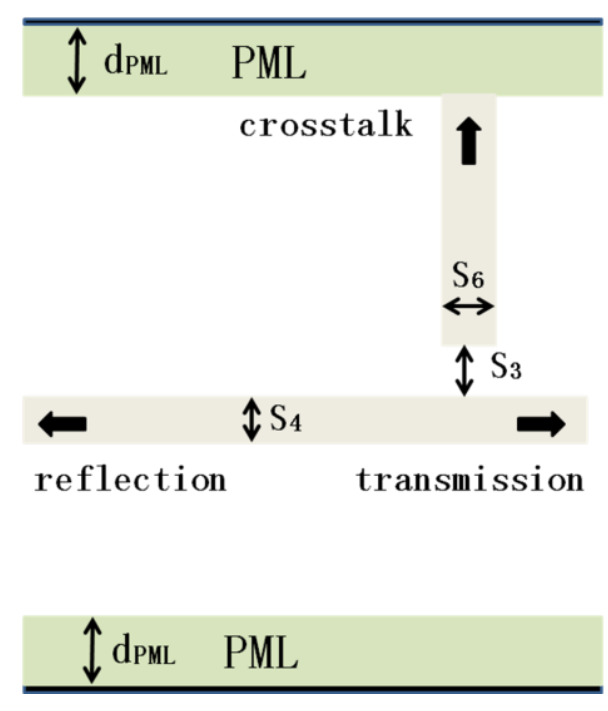

(a)

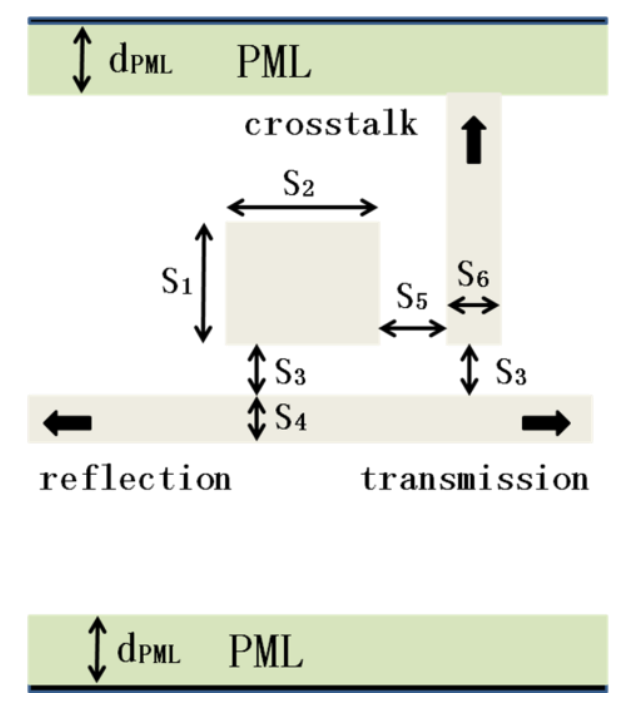

(b)

Figure 6.17. (a)The schematic of a 2-D waveguide T-junction (b) The schematic of a cavity-assisted waveguide T-junction. $\mathrm{dPML}=5 \mu \mathrm{m}, \mathrm{S} 1=0.6 \mu \mathrm{m}, \mathrm{S} 2=0.6 \mu \mathrm{m}, \mathrm{S} 3=20 \mathrm{~nm}$, $\mathrm{S} 4=0.2 \mu \mathrm{m}, \mathrm{S} 5=20 \mathrm{~nm}, \mathrm{~S} 6=0.2 \mu \mathrm{m}$.

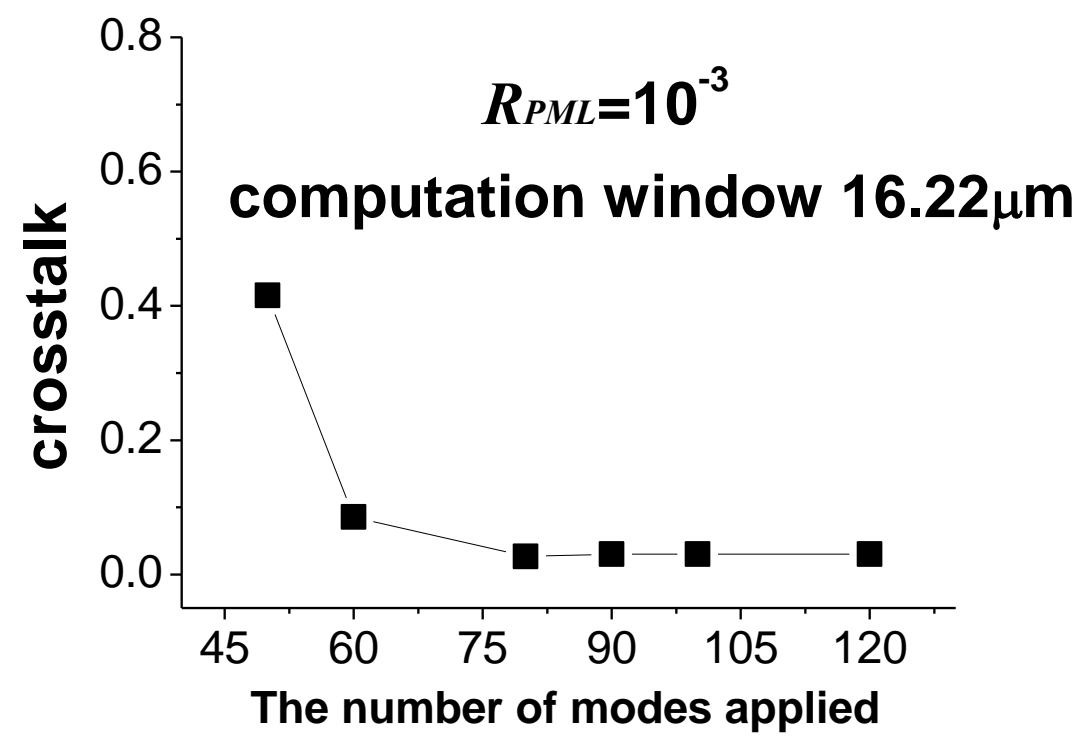

(a) 


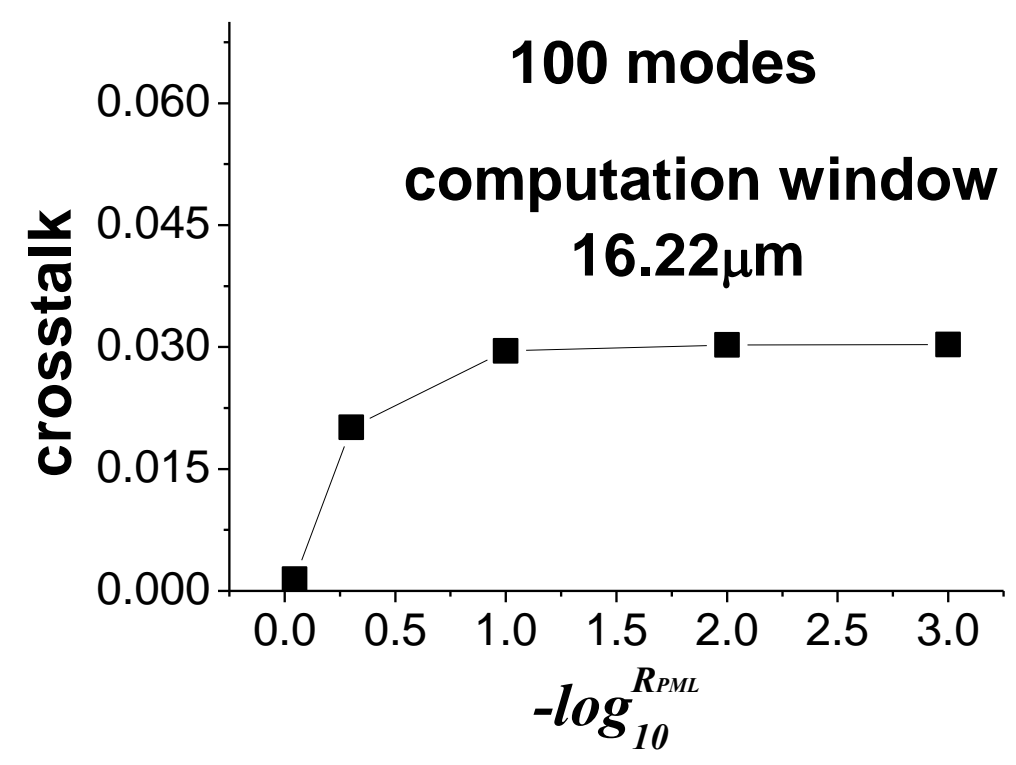

(b)

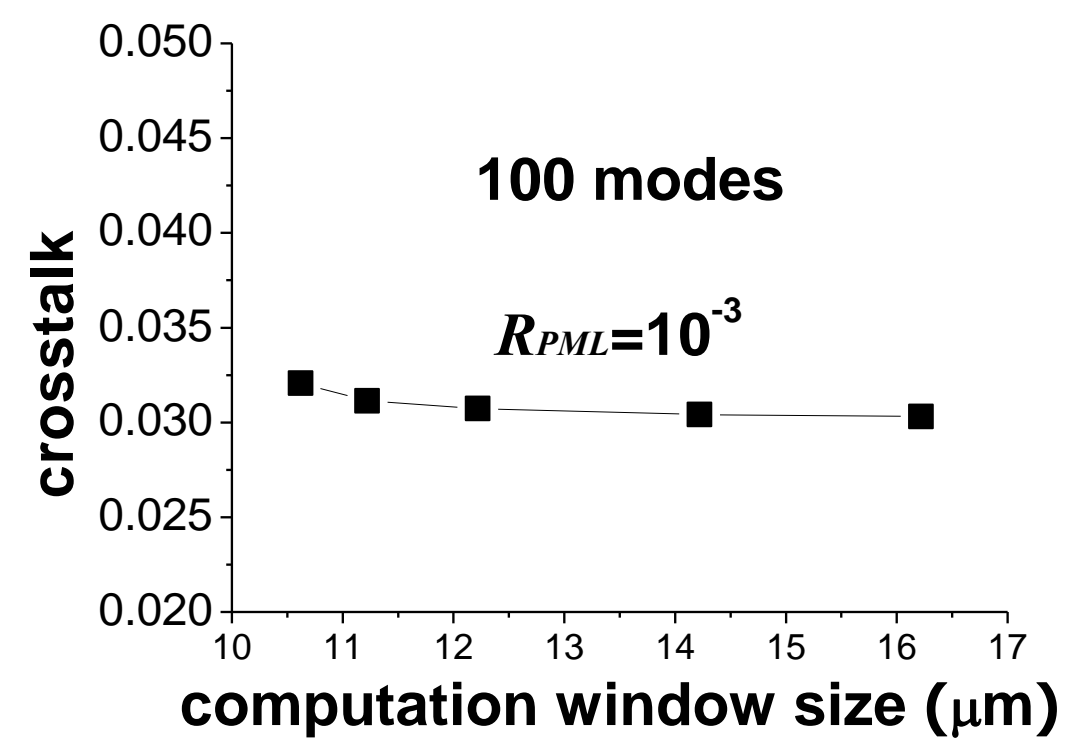

(c)

Figure 6.18. Convergence test of guided crosstalk about (a) the number of modes applied (b) $R_{P M L}$ and (c) the computation window in waveguide T-junction (Figure 6.28(a)) 


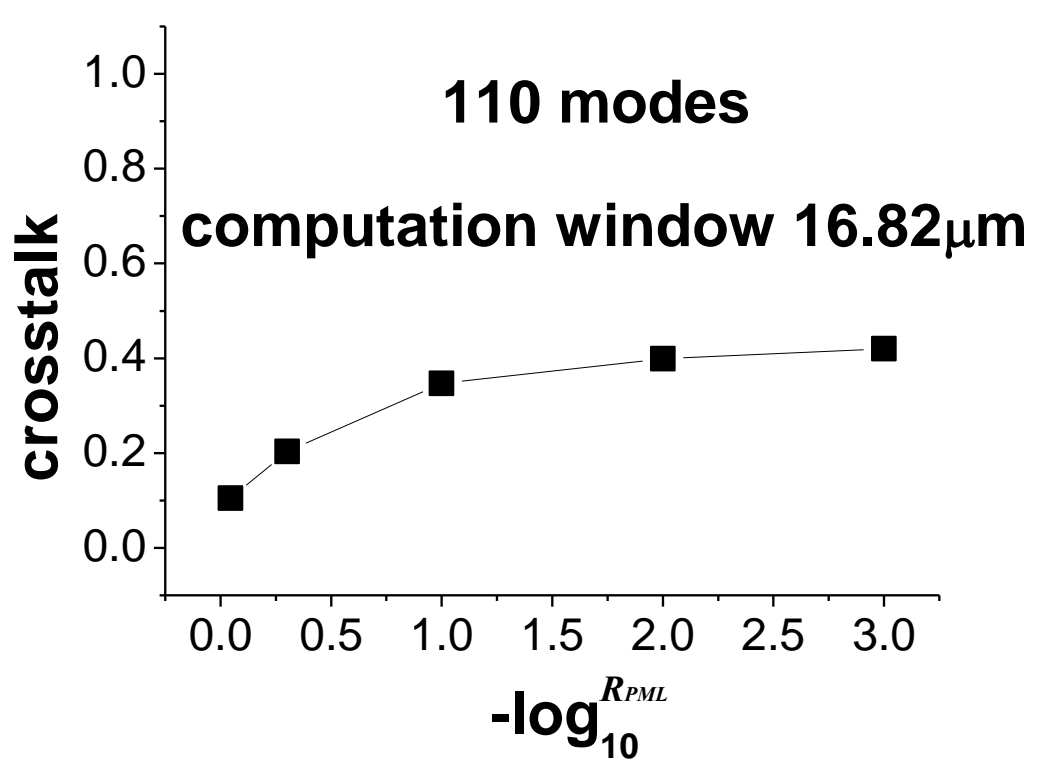

(a)

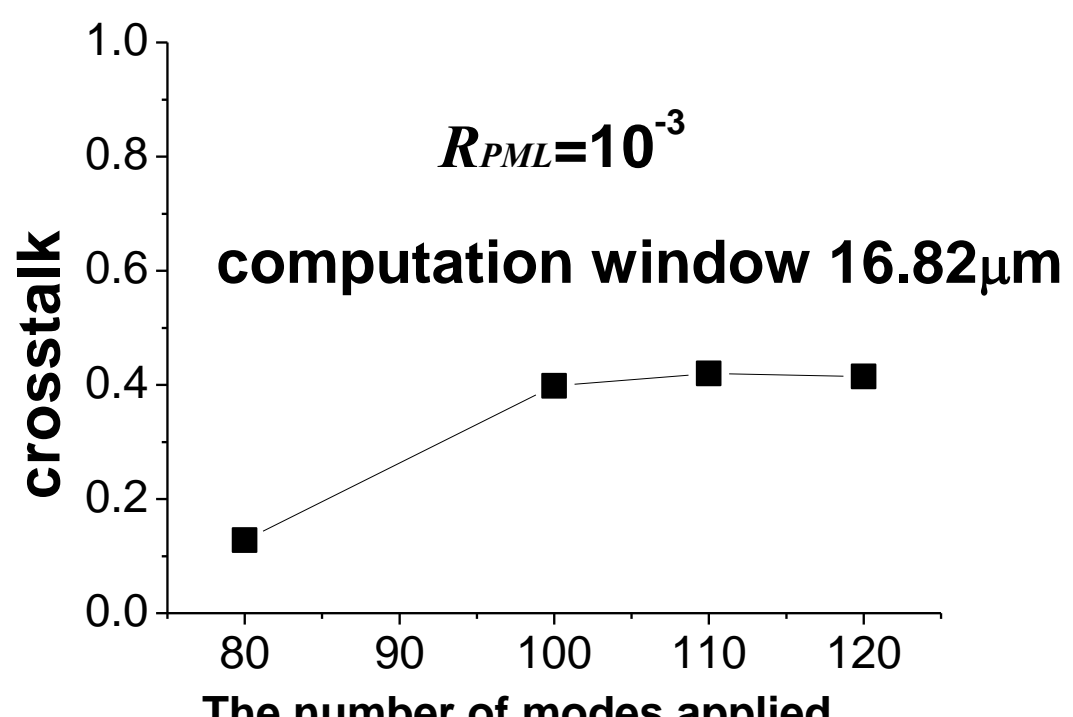

The number of modes applied

(b) 


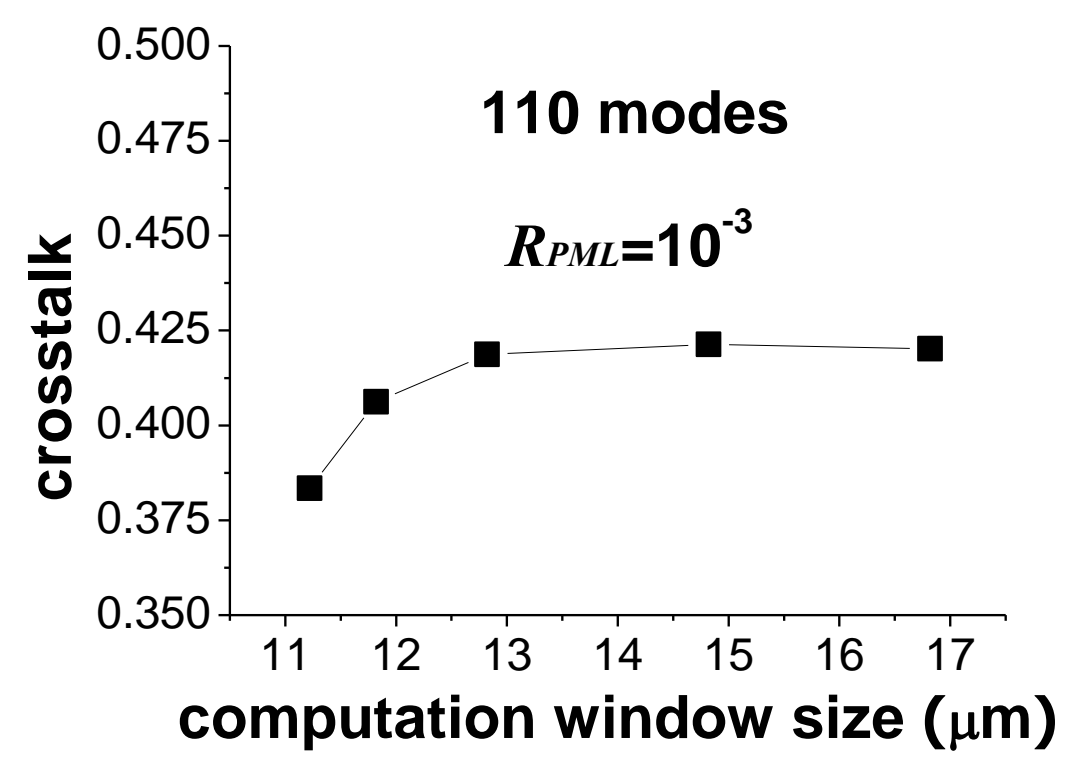

(c)

Figure 6.19. Convergence test of guided crosstalk about (a) $R_{P M L}$ (b) the number of modes applied, and (c) the computation window in cavity-assisted waveguide T-junction (Figure 6.17(b))

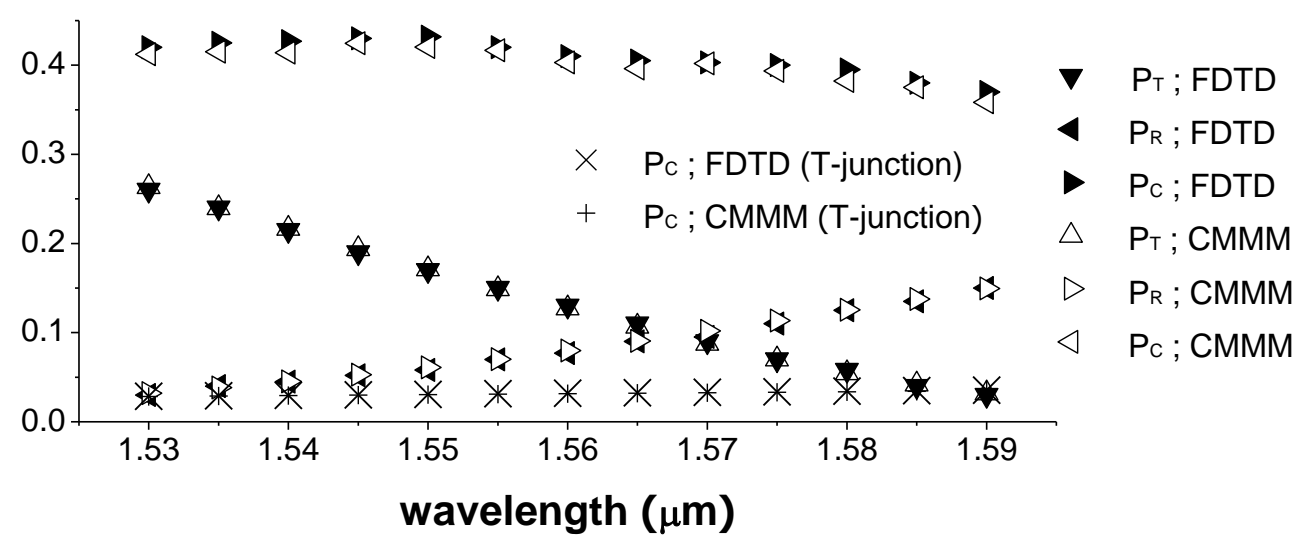

Figure 6.20. Guided transmission, reflection and crosstalk spectra of the cavity-assisted T-junction (Figure 6.17(b)), and guided crosstalk spectra of the T-junction (Figure 6.17(a)) 


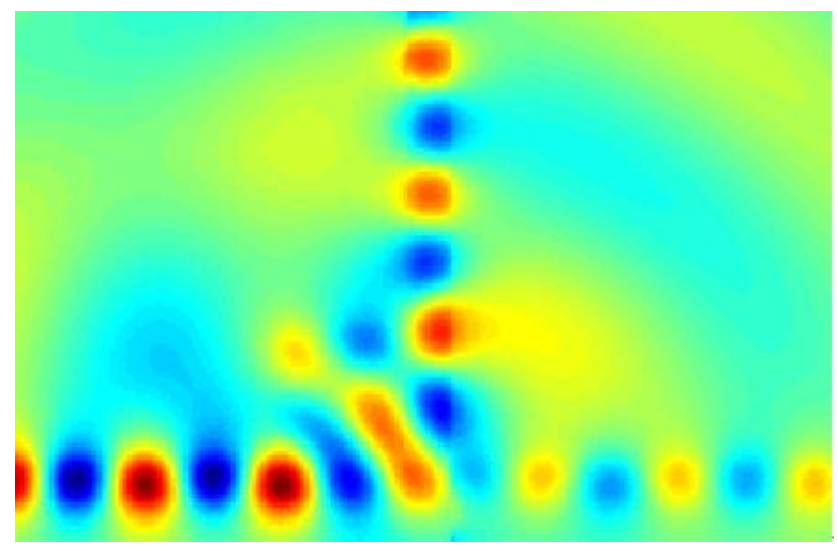

(a)

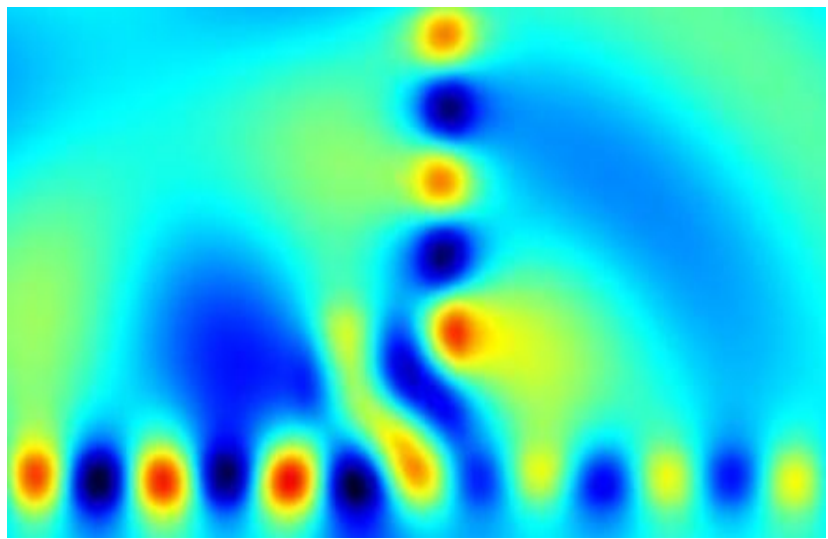

(b)

Figure 6.21. Electric field amplitude pattern in cavity-assisted T-junction (Figure 6.17(b)) (a) CMMM (b) FDTD

\subsection{The Validation of CMMM with QDEEM in Cross Waveguide}

QDEEM is another frequency-domain scheme for the simulation of waveguide interconnects. The 2-D waveguide is terminated with PML and PRB not only vertically but also horizontally in the framework of QDEEM. We follow the case of waveguide crossing in [38] and prove that the modeling based on CMMM creates the coinciding results with those from QDEEM. PD (PU), PR and PT are defined in [38] as the guided scattering, transmitted and reflected power in the waveguide crossing, 
respectively. With dPML on each side as $5 \mu \mathrm{m}$ and the mesh size as $20 \mathrm{~nm}$, Figure 6.22 and 6.23 show the convergence tests in TE and TM, respectively, when $v$ is $0.2 \mu \mathrm{m}$ and the wavelength is $1.55 \mu \mathrm{m}$.

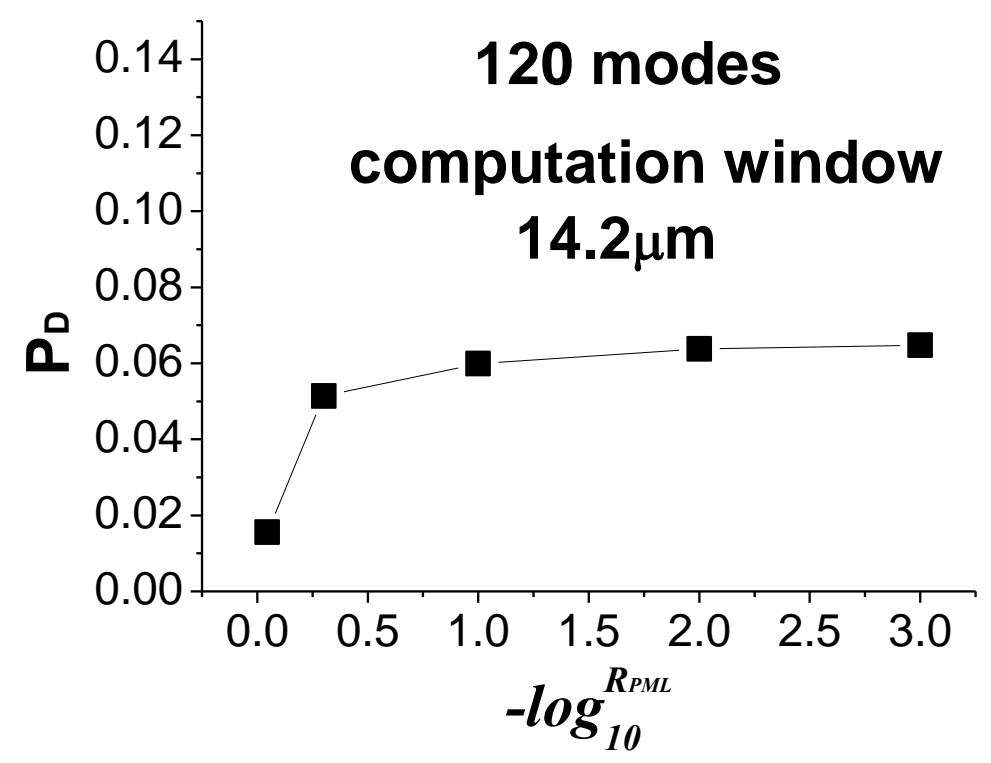

(a)

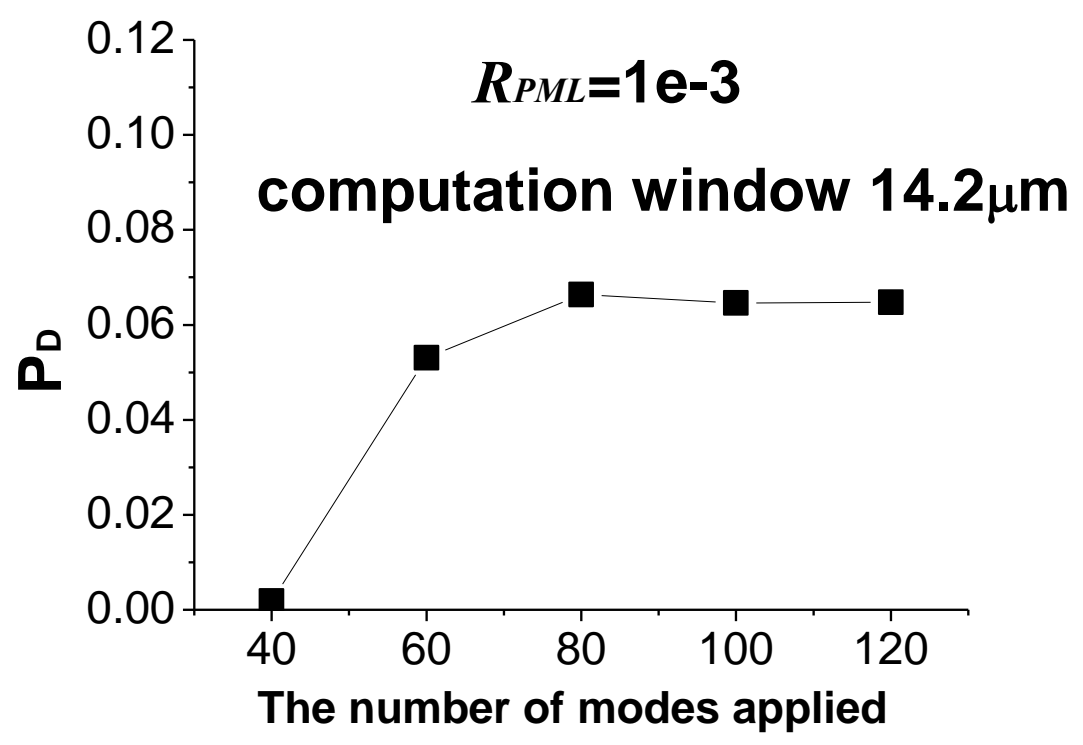

(b) 


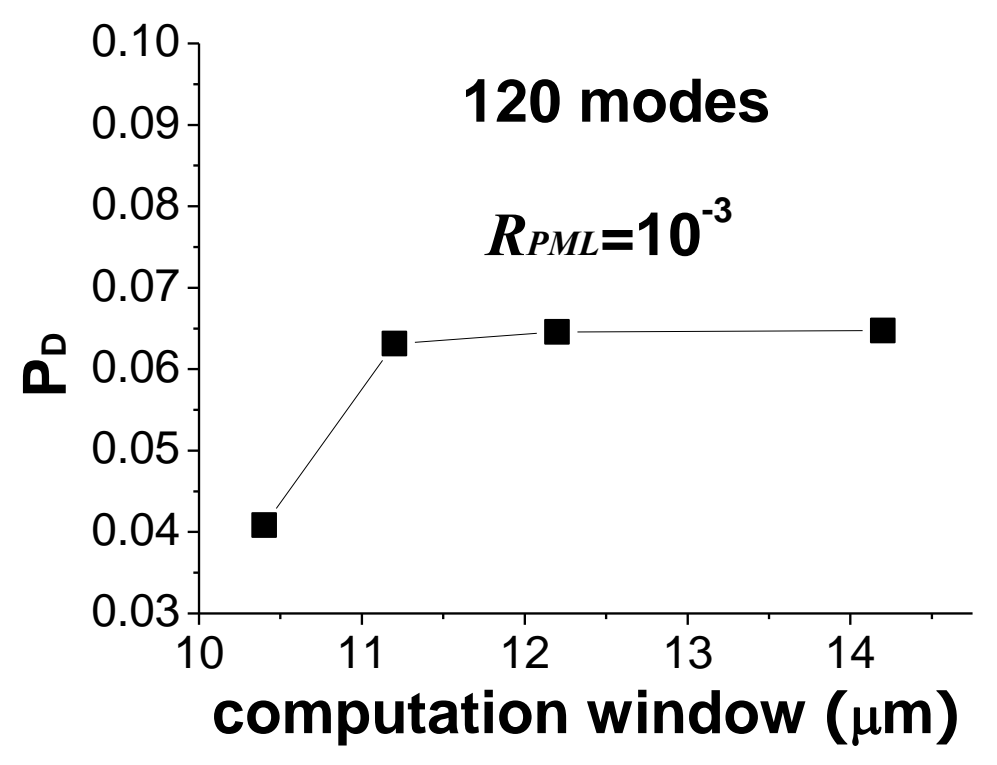

(c)

Figure 6.22.Convergence test of $\mathrm{P}_{\mathrm{D}}$ about (a) $R_{P M L}$ (b) the number of modes applied , and (c) the computation window for the waveguide crossing ([38]) in TE polarization.

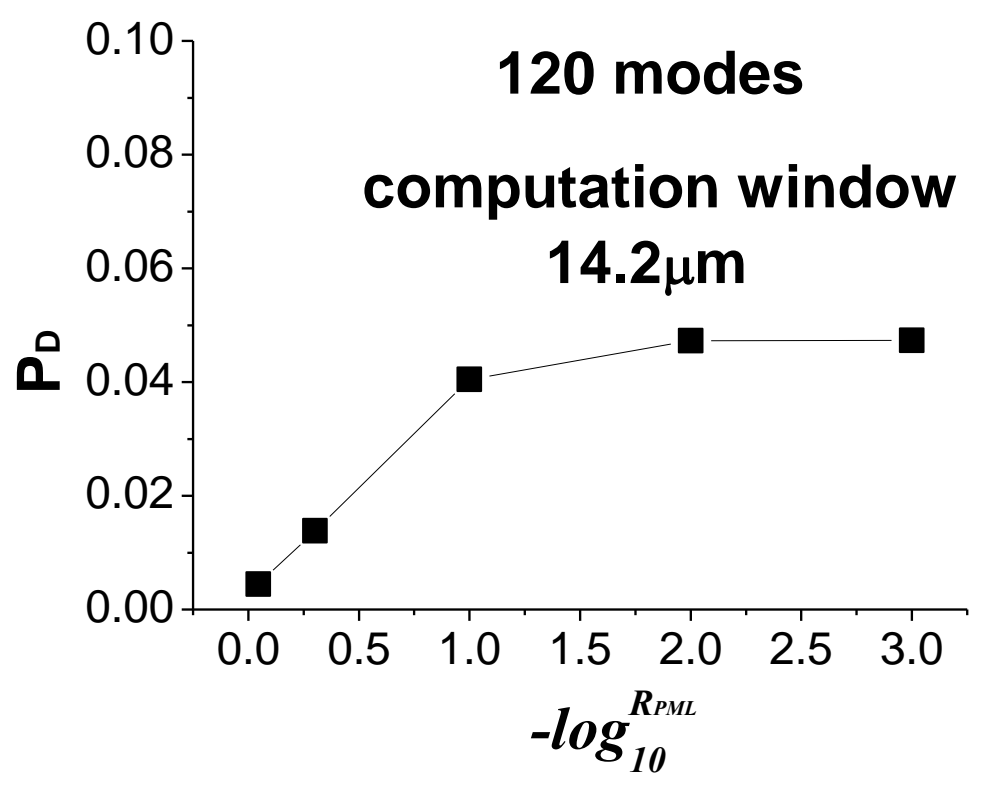

(a) 


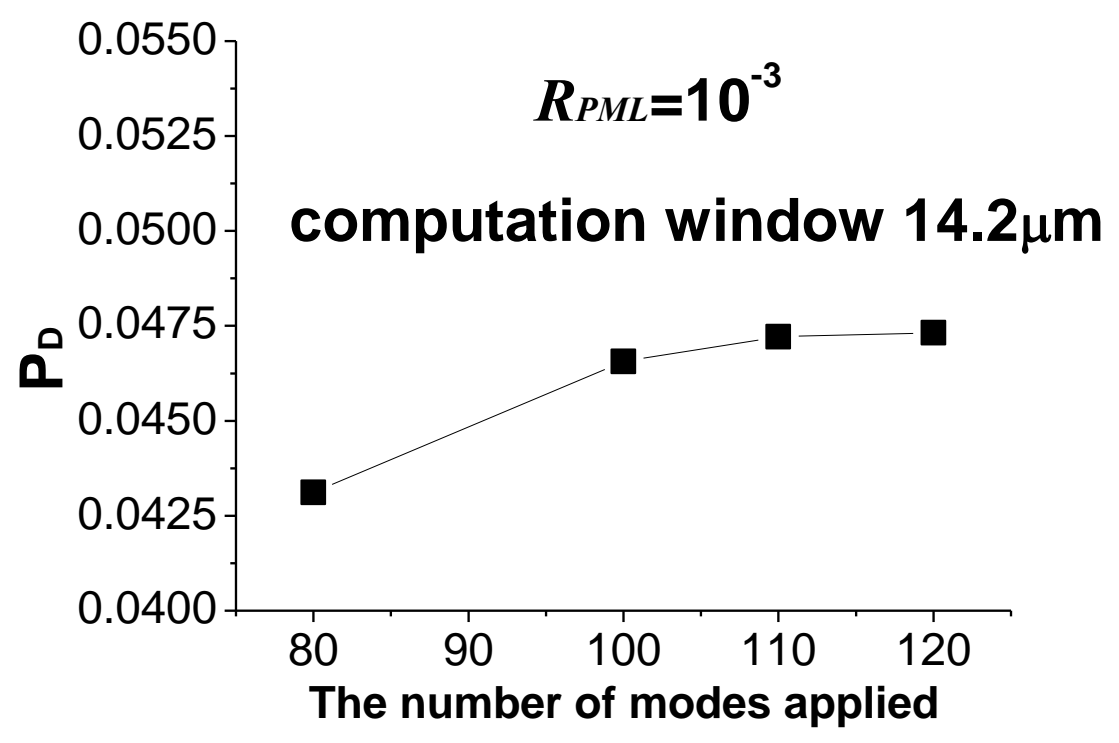

(b)

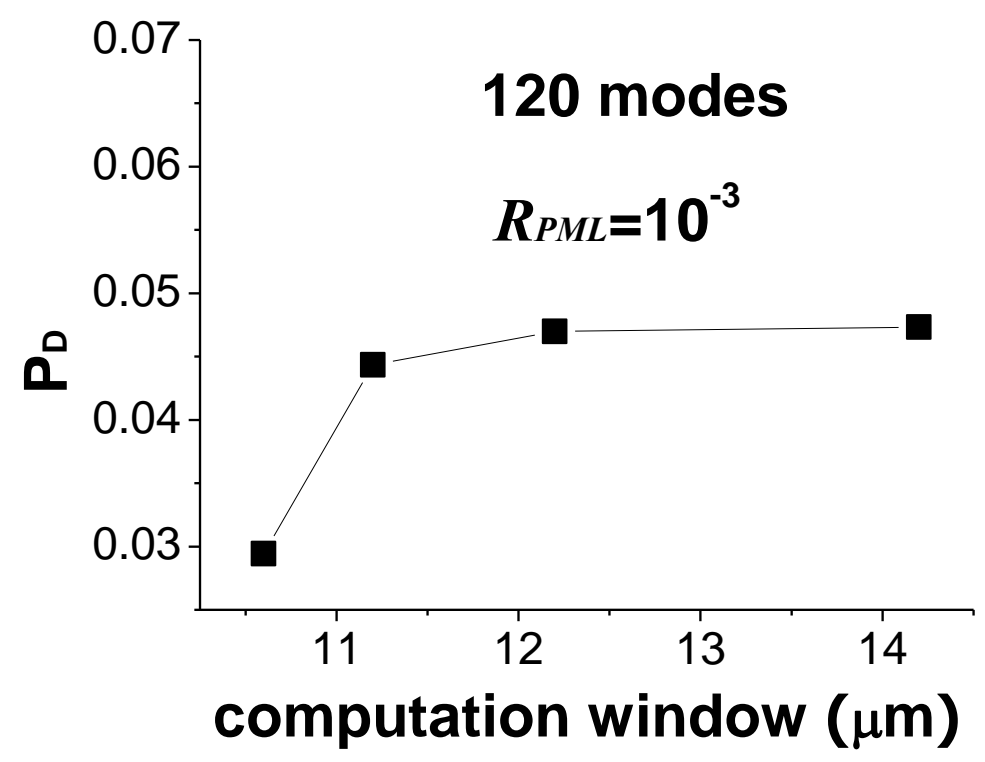

(c)

Figure 6.23. Convergence test of $\mathrm{P}_{\mathrm{D}}$ about (a) $R_{P M L}$ (b) the number modes of applied, and (c) the computation window for the waveguide crossing ([38]) in TM polarization case.

After the convergence tests, with $R_{P M L}$ as 1e-3, 120 modes considered and the computation window as $14.2 \mu \mathrm{m}$, we study the evolution of $\mathrm{P}_{\mathrm{D}}, \mathrm{P}_{\mathrm{T}}, \mathrm{P}_{\mathrm{R}}$ and $\mathrm{P}_{\mathrm{U}}$ with 
the variation of $v$ in both TE and TM polarization cases in Figure 6.24. Our results coincide well with those obtained from EEM in [38].

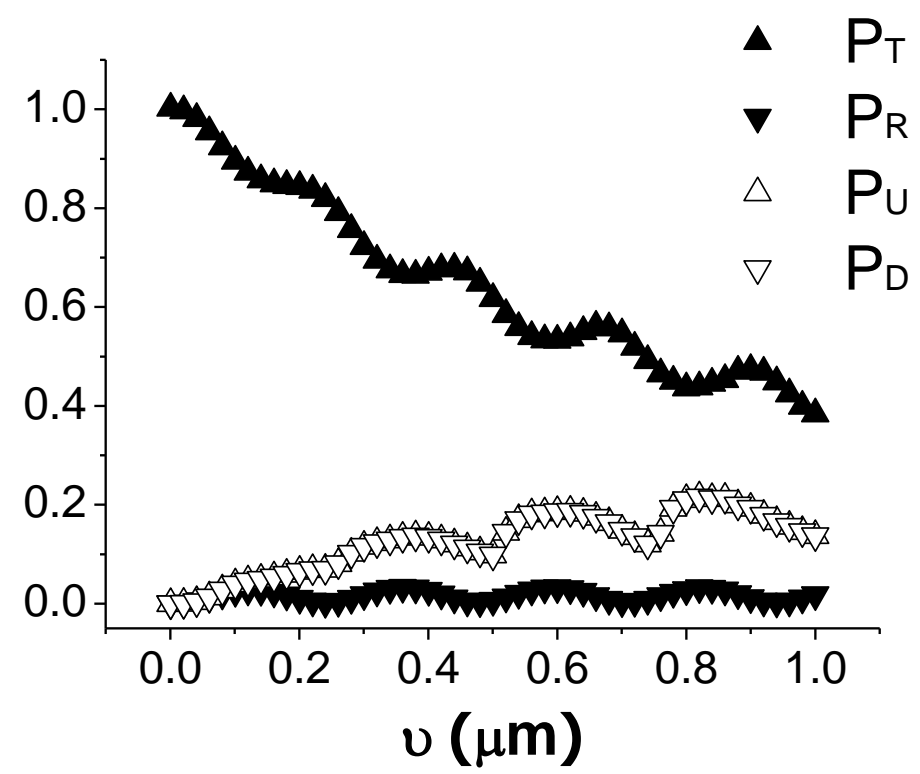

(a)

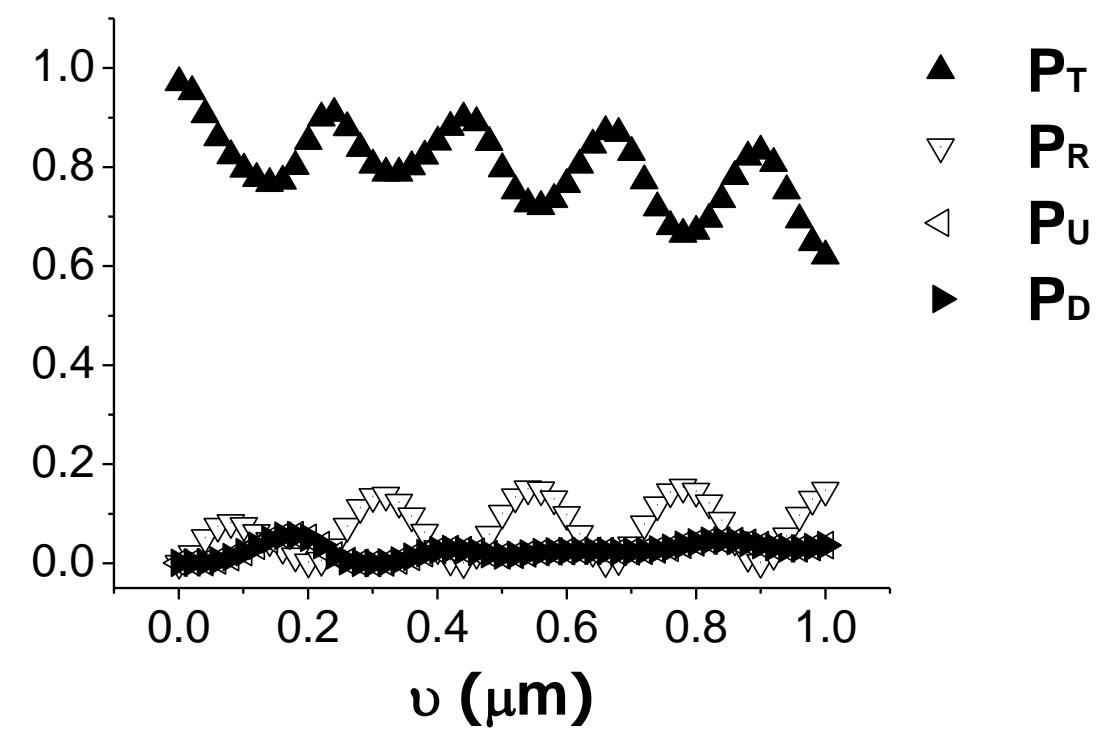

(b)

Figure 6.24. The evolutions of guided $\mathrm{P}_{T}, \mathrm{P}_{\mathrm{R}}, \mathrm{P}_{\mathrm{U}}$ and $\mathrm{P}_{\mathrm{D}}$ with the variation of $v$.(a) TE (b) $\mathrm{TM}$ 


\section{Chapter 7}

\section{Conclusions}

The thesis introduces several simulation schemes for the calculation of mode profiles and wave propagation in multi-layer planar waveguide. The mode spectrums and modal patterns of slab waveguide, hollow waveguide and leaky waveguide are obtained with semi-analytical Complex STM facilitated by PML and PRB, and they coincide with those from HOFD scheme. Without the troublesome procedures of getting eigenvectors of the Hermitan matrices in HOFD, Complex STM performs more efficiently than HOFD, especially in the case of sophisticated waveguide structures in which very small mesh is necessary for HOFD.

Power conservation in the framework of CMMM is proved analytically in hollow waveguide and numerically in leaky waveguide and around waveguide junction. Waveguide crossings and corners with high-index-contrast are simulated with CMMM and further validated by FDTD. With the background index varying and the waveguide core's dimension extending, the accuracy of CMMM is still held. Additionally, the high-index-contrast T-junction incorporating a high-index resonant cavity shows the enhancement of crosstalk in a broad range of wavelength by CMMM. Again the spectra calculated through CMMM coincide with those of FDTD. At last, 
CMMM is proved to produce nearly the same results with QDEEM in the examples of high-index-contrast waveguide crossing. All these evidences support the conclusion that CMMM is capable of modeling the couplings of radiating wave perpendicular to the waveguide axis. 


\section{Bibliography}

[1]Vivek Alwayn, "Optical network design and implement," Indianapolis, Cisco Press, 2004.

[2] L.G. Kazovsky, W.T. Shaw, D. Gutierrez, and S.W. Wong, "Next-generation broadband optical access networks," IEEE/OSA Journal of Lightwave Technology, vol.25, pp.3428-3442, 2007.

[3] S.E.Miller, "Integrated optics: an introduction," Bell Sys. Tech. Journal, vol.48, pp.2059-2069, 1969.

[4] S.-H. Yen, S.-W. Wong, S. Das, Ning Cheng, Jinwoo Cho, S. Yamashita, O. Solgaard, and L.G. Kazovsky, "Photonic components for future fiber access networks," IEEE Journal on Selected Areas in Communications, vol.28, pp.928-935, 2010.

[5] Fang $\mathrm{Xu}$, and Andrew W. Poon, "Silicon cross-connect filters using microring resonator coupled multimode-interference-based waveguide crossings," Optics Express, vol.16, pp.8649-8657, 2008.

[6] Universität Dortmund, and Lehrstuhl für Hochfrequenztechnik, "Low-crosstalk passive polarization splitters using Ti:LiNbO3 waveguide crossings," Applied Physics Letter, vol.55, pp.927-929, 1989.

[7] Hui Chen and Adrew W. Poon, "Low-loss multimode-interference-based crossings for silicon wire waveguides," Photonics Technology Letter, vol.18, pp.2260-2262, 2006.

[8] Sanchis P., Galan J.V., Griol A., Marti J., Piqueras M.A., and Perdigues J.M., "Low-crosstalk in silicon-on-insulator waveguide crossings with optimized-angle," Photonic Technology Letter, vol.19, pp.1583-1585, 2007.

[9] D.Marcuse, "Theory of dielectric optical waveguides," New York, Academic Press, Inc., 1974267 p., 1974.

[10] Shun Lien Chuang, "Physics of photonics devices," Wiley, 2nd edition, 2009.

[11] C. Manolatou, Steven G. Johnson, Shanhui Fan, Pierre R. Villenjeuve, H. A. Haus, and J.D. Joannopoulos, "High-density integrated optics," Journal of Lightwave Technology, vol.17, pp.1682-1692, 1999.

[12] Steven G. Johnson, Christina Manolatou, Shanhui Fan, Pierre R. Villeneuve, J.D. Joannopoulos, and H. A. Haus, "Elimination of cross talk in waveguide intersections, " Optics Letters, vol. 23, pp.1855-1857, 1998.

[13] Wim Bogaerts, Pieter Dumon, Dries Van Thourhout, and Roel Baets, "Low-loss, low-cross-talk crossings for silicon-on-insulator nanophotonic waveguides," Optics Letters, vol.32, pp.2801-2803, 2007. 
[14] Neyer A., Mevenkamp W., Thylen L., and Lagerstrom B., "A beam propagation method analysis of active and passive waveguide crossings," Journal of Lightwave Technology, vol.3, pp. 635-642, 1985.

[15] Roberts D. A., Rahm M., Pendry J. B., and Smith D. R., "Transformation-optical design of sharp waveguide bends and corners," Applied Physics Letters, vol. 93, pp.251111-251111-3, 2009.

[16] R. L. Espinola, R. U. Ahmad, F. Pizzuto, M. J. Steel, and R. M. Osgood, "A study of high-index-contrast of 90 degree waveguide bend structures," Optics Express, vol.8, pp. 517-528, 2001.

[17] G. Ronald Hadley, "High-accuracy finite-difference equations for dielectric waveguide analysis II: dielectric corners, ”Journal of Lightwave Technology, vol. 20, pp.1219-1231, 2002.

[18] G. P. Karman, "Laser optics: fractal modes in unstable resonators," Nature, vol.402, pp.138, 1999.

[19] Justin C. Johnson, Haoquan Yan, Peidong Yang, and Richard J. Saykally, "Optical cavity effect in $\mathrm{ZnO}$ nanowire lasers and waveguides," Journal of Physical Chemistry B, vol.107, pp.8816-8828, 2003.

[20] Schubert E. F., Wang Y. H., Cho A. Y., Tu Y. W., and Zydzik G. J., "Resonant cavity light-emitting diodes," Applied Physics Letters, vol.60, pp.921-923, 1992.

[21] Lang R. and Kobayashi K., "External optical feedback effects on semiconductor injection lasers properties," Journal of Quantum Electronics, vol.16, pp.347-355, 1980.

[22] Simon Grolacher, Klemens Hammerer, Michael R. Vanner, and Markus Aspelmeyer, "Observation of strong coupling between micromechanical resonator and an optical cavity field," Nature, vol. 460, pp.724-727, 2009.

[23] P. Grangier, E. Slusher, B. Yurke, and A. LaPorta, "Squeezed-light-enhanced polarization interferometer," Physics Review Letter, vol.59, pp.2153-2156, 1987.

[24] Tayebati P., Wang P., Azimi M., Maflah L., and Vakhshoori D., "Microelectromechanical tunable filter with stable half symmetric cavity," Electronic Letters, vol.34, pp.1967-1968, 1998.

[25] William Gunning, "Double-cavity electrooptic Fabry-Perot tunable filter," Applied Optics, vol.21, pp.3129-3131, 1982.

[26] Chung Yan Fong and Andrew W. Poon, "Planar corner-cut square microcavities: ray optics and FDTD analysis," Optics Express, vol.12, pp.4864-4874, 2004.

[27] H. A. Haus, W.P. Huang, S. Kawakami, and N. A. Whitaker, "Coupled-mode theory of optical waveguides," Journal of Lightwave Technology, vol.LT-5, pp.16-23, 1987.

[28] Weiping Huang, "Coupled-mode theory for optical waveguides: an overview," Journal of Optical Society of America A, vol. 11, pp. 963-983, 1994.

[29] Y. C. Lu, W. -P. Huang, and S. S. Jian, "Full vector complex coupled mode theory for tilted fiber gratings," Optics Express, vol. 18, pp. 713-U366, 2010.

[30] W. -P. Huang and J. Mu, "Complex coupled-mode theory for optical waveguides," Optics Express, vol. 17, pp.19134-19152, 2009. 
[31] Y. C. Lu, L. Yang, and W. -P. Huang, "Improved finite difference full vector domplex mode solver for optical waveguides of circular symmetry," IEEE/OSA Journal of Lightwave Technology, vol. 26, pp.1868-1876, 2008.

[32] J. Mu and W. Huang, "Simulation of three-dimensional waveguide discontinuities by a full-vector mode-matching method based on finite-difference schemes," Optics Express, vol.16, pp.18152-18163, 2008

[33] M. D. Feit and J. A. Fleck, "Light propagation in graded-index optical fibers," Applied Optics, vol.17, pp.3990-3998, 1978.

[34] W.P. Huang, C. L. Xu, S. T. Cu, and S. K. Chaudhuri, "A finite difference vector beam propagation method: analysis and assessment," IEEE Journal of Lightwave Technology, vol.10, pp.295-305, 1992.

[35] S.T. Chu and S. K. Chaudhuri, "A finite-difference time-domain method for the design and analysis of guided-wave optical structures," IEEE Journal of Lightwave Technology, vol.7, pp.2033-2038, 1989.

[36] W.P. Huang, S. T. Chu, A. Goss, and. S. K. Chaudhuri, “A scalar finite-difference time-domain approach to guided wave optics," IEEE Photonics Technology Letter, vol.3, pp.524-526, 1991.

[37] A. Taflove and S. C. Hagness, "Computational electrodynamics: the finite-difference time-domain method," 3rd ed. Boston: Artech House, 2005.

[38] Manfred Hammer, "Quadridirectional eigenmode expansion scheme for 2-D modeling of wave propagation in integrated optics," Optics Communications, vol.235, pp.285-303, 2004.

[39] Yih-Peng Chiou, Yen-Chung Chiang, and Hung-Chun Chang, “Improved three-point formulas considering the interface conditions in the finite-difference analysis of step-index optical devices, J Journal of Lightwave Technology, vol.18, pp.243-251, 2000.

[40] Ali Khalatpour, Jianwei Mu, Kaveh Moussakhani, and Weiping Huang, "Modified smooth transition method for determination of complex modes in multilayer waveguide structures," Journal of Lightwave Technology, vol.28, pp.2851-2855, 2010.

[41] J. Chillwell and I. Hodgkinson, "Thin-films field-transfer matrix theory of planar multi-layer waveguides and reflection from prism-loaded waveguide,” Journal of Optical Society of America A, vol.1, pp.742-753, 1984.

[42] J. P. Berenger, "A perfectly matched layer for the absorption of electromagnetic-waves," Journal of Computational Physics, vol.114, pp.185-200, 1994.

[43] W.P. Huang, C.L. Xu, W. Lui, and K. Yokoyama, “The perfectly matched layer (PML) bounary condition for the beam propagation method," Photonics Technology Lettes, vol.8, pp.649-651, 1996.

[44] H.Rogier and D. De Zutter, "Berenger and leaky modes in microstrip substrates terminated by a perfectly matched layer," IEEE Transactions on Microwave Theory and Techniques, vol.49, pp.712-715, 2001.

[45] S. S. A. Obayya, B. M. A. Rahman, K. T. V. Grattan, and H. A. EI-Mikati, "Full vectorial finite-element-based imaginary distance beam propagation solution of 
complex modes in optical waveguides," Journal of Lightwave Technology, vol.20, pp.1054-1060, 2002.

[46] B. Rahman and J. Davies, "Finite-element solution of integrated optical waveguides,” Journal of Lightwave Technolog, vol.2, pp.682-688, 1984.

[47] U. Rogge and R. Pregla, "Method of lines for the analysis of integrated optical wavegudies," Journal of Lightwave Technology, vol.11, pp.2015-2020, 1993.

[48] U. Rogge and R.Pregla, "Method of lines for the analysis of strip-loaded optical waveguides," Journal of Optical Society of America B, vol.8, pp.459-463, 1991.

[49] M. S. Stern, "Semivectorial Polarized H Field Solutions for Dielectric Waveguides with Arbitrary Index Profiles, ” IEE Proceedings-Journal of Optoelectronics, vol.135, pp.333-338, 1988.

[50] W. E. Arnoldi, “The principle of minimized iterations in the solution of the matrix eigenvalue problem," Journal of Applied Math, vol.9, pp.17-19, 1951.

[51] Hill K. O. and Meltz G., "Fiber Bragg grating technology fundamentals and overviews,” Journal of Lightwave Technology, vol.15, pp.1263-1276, 1997.

[52] Henk Derudder, Frank Olyslager, Daniel De Zutter and Steve Van den Berghe, "Efficient mode-matching analysis of discontinuities in finite planar substrate using perfectly matched layers," IEEE Transaction on Antennas and Propagation,vol.49, pp.185-195, 2001.

[53] Jianwei Mu and Weiping Huang, "Simulation of three-dimensional waveguide discontinuities by a full-vector mode-matching method based on finite-difference schemes," Optics Express, vol.16, pp.18152-18163, 2008. 


\section{Appendix A \\ List of Publications}

[1]Rui Wang, Lin Han, Jianwei Mu, and Weiping Huang, "Simulation of waveguide corner and cross with Complex Mode Matching Method," Integrated Photonics Research, Silicon and Nanophotonics, paper IMC 6, Toronto, Canada, 2011. 